TESTING GENERAL RELATIVITY IN THE STRONG-FIELD REGIME WITH OBSERVATIONS OF BLACK HOLES IN THE ELECTROMAGNETIC SPECTRUM

by

Tim Johannsen

A Dissertation Submitted to the Faculty of the DEPARTMENT OF PHYSICS

In Partial Fulfillment of the Requirements

For the Degree of

DOCTOR OF PHILOSOPHY

In the Graduate College

THE UNIVERSITY OF ARIZONA

2012 


\section{THE UNIVERSITY OF ARIZONA GraduAte College}

As members of the Dissertation Committee, we certify that we have read the dissertation prepared by Tim Johannsen entitled Testing General Relativity in the Strong-Field Regime with Observations of Black Holes in the Electromagnetic Spectrum and recommend that it be accepted as fulfilling the dissertation requirement for the Degree of Doctor of Philosophy.

Dimitrios Psaltis

Date: 23 April 2012

Date: 23 April 2012

Daniel Marrone

Date: 23 April 2012

Feryal Özel

Date: 23 April 2012

Kenneth Johns

Final approval and acceptance of this dissertation is contingent upon the candidate's submission of the final copies of the dissertation to the Graduate College.

I hereby certify that I have read this dissertation prepared under my direction and recommend that it be accepted as fulfilling the dissertation requirement.

Dissertation Director: Dimitrios Psaltis

Date: 23 April 2012 


\section{STATEMENT By AUthor}

This dissertation has been submitted in partial fulfillment of requirements for an advanced degree at The University of Arizona and is deposited in the University Library to be made available to borrowers under rules of the Library.

Brief quotations from this dissertation are allowable without special permission, provided that accurate acknowledgment of source is made. Requests for permission for extended quotation from or reproduction of this manuscript in whole or in part may be granted by the head of the major department or the Dean of the Graduate College when in his or her judgment the proposed use of the material is in the interests of scholarship. In all other instances, however, permission must be obtained from the author.

SIGNED: Tim Johannsen 


\section{ACKNOWLEDGMENTS}

Many are the names of those who have contributed to my time as a graduate student at the University of Arizona and to the research presented in this thesis. Without them, many projects would have remained unconceived or unfinished, and I am grateful for their help along the way.

First of all, I would like to thank Dimitrios Psaltis, my thesis advisor, for his guidance, support, inspiration, and encouragement that have been invaluable. I greatly enjoyed our many discussions on astrophysics as well as on black holes and their properties. I am very grateful for the conferences that I have been able to attend and the talks that I could give. I also valued his practical advice on the many different aspects of science. I likewise thank Feryal Özel, who has taught me a lot about compact objects and high-energy astrophysics, and Dan Marrone for our discussions on VLBI and Sgr A*.

I thank Tolga Güver, with whom I have shared an office, for many conversations and the observations that we performed from Kitt Peak. I also thank the other members of our theoretical astrophysics group for many good discussions, escpecially Alan Cooney and Michi Bauböck.

It is my pleasure to thank my other collaborators including Avery Broderick, Shep Doeleman, Vincent Fish, Stefan Gillessen, Scott Hughes, Henric Krawczynski, Avi Loeb, Sera Markoff, Jeff McClintock, Jon McKinney, Ramesh Narayan, Jeremy Schnittman, Jack Steiner, Phil Uttley, Sarah Vigeland, Bob Wagoner, Cliff Will, Jörn Wilms, and Nicolás Yunes.

Last but not least, I thank Enrique Montaño for the many the homework problems we solved together during our first two years as graduate students. 


\section{DEDICATION}

"There is no logical way to the discovery of these elemental laws. There is only the way of intuition, which is helped by a feeling for the order lying behind the appearance."

Albert Einstein 
TABLE OF CONTENTS

LIST OF FIGURES . . . . . . . . . . . . . . . . . . . . . . . . . . . . 10

LIST OF TABLES . . . . . . . . . . . . . . . . . . . . . . . 13

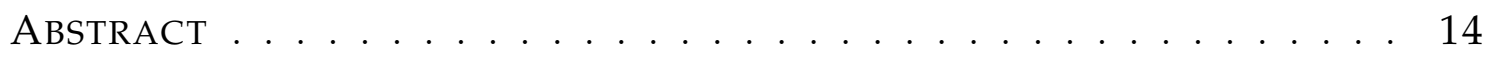

CHAPTER 1 IntRODUCTION . . . . . . . . . . . . . . . . . . 16

1.1 Tests of the Foundations of the Gravitation Theory . . . . . . . . 17

1.2 Weak-Field Tests of General Relativity . . . . . . . . . . . . . . 20

1.2.1 Light Deflection and Shapiro Time Delay . . . . . . . . . . 21

1.2.2 The Perihelion Precession of Mercury . . . . . . . . . . . 23

1.2 .3 Gravitomagnetism . . . . . . . . . . . . . . 25

1.2.4 Timing Observations of Double Pulsars . . . . . . . . . . 26

1.3 Testing General Relativity in the Strong-Field Regime . . . . . . . 29

1.4 Testing the No-Hair Theorem . . . . . . . . . . . . . . . 34

1.4.1 Violations of the No-Hair Theorem . . . . . . . . . . . . 35

1.4.2 Prediction of Observational Signatures of Violations of the No-Hair Theorem . . . . . . . . . . . . . . . . . . 40

1.5 Constraining the Size of Large Extra Dimensions . . . . . . . . . . 49

Chapter 2 A Framework for Testing the No-Hair Theorem . . . . 53

2.1 Multipole Moments and the No-Hair Theorem . . . . . . . . . . 55

2.1.1 Multipole Expansions of Curved Spacetimes . . . . . . . . . 55

2.1.2 Four Approaches to Measuring the Multipole Moments of Curved Spacetimes . . . . . . . . . . . . . . . . . 57

2.2 Defining a Framework For Testing the No-Hair Theorem . . . . . . 63

2.3 Properties of the Quasi-Kerr Metric . . . . . . . . . . . . . . . . . . . 69

2.3.1 The Event Horizon and Static Limit . . . . . . . . . . . . . 69

2.3.2 The Innermost Stable Circular and Marginally Bound Orbits 70

2.3.3 Photon Trajectories . . . . . . . . . . . . . . . . 73

2.3.4 Gravitational Redshift and Lorentz Boosts . . . . . . . . 76

2.3.5 The Circular Photon Orbit . . . . . . . . . . . . . . . . . . 79

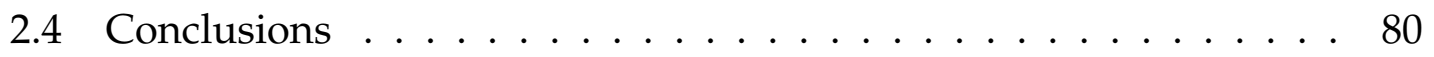

Chapter 3 Systematic Study of Pathologies of Black Hole MetRiCS THAT Violate the No-Hair THEOREM . . . . . . . . . . . . . . 83

3.1 Parametric Deviations from the Kerr Metric . . . . . . . . . . . . 84

3.1.1 The Quasi-Kerr Metric . . . . . . . . . . . . . . . . . . . 84 


\section{TABLE OF CONTENTS - Continued}

3.1.2 The Bumpy Kerr Metric _ . . . . . . . . . . . . . . . . . 85

3.1.3 The Ricci flat metric proposed by Manko \& Novikov . . . . 87

3.1.4 The Modified Gravity Bumpy Kerr Metric . . . . . . . . . . 89

3.2 Properties of the Spacetimes . . . . . . . . . . . . . . . . . . 92

3.2.1 Conserved Quantities and Petrov Type . . . . . . . . . . 92

3.2 .2 Asymptotic Flatness . . . . . . . . . . . . . . . . . . 93

3.2.3 Singularities and the Event Horizon . . . . . . . . . . . . 95

3.2.4 Lorentz Violations, Closed Timelike Curves, and Regions of Validity . . . . . . . . . . . . . . . . . . 100

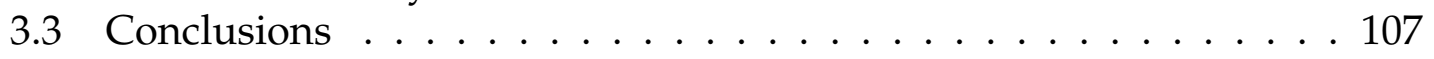

Chapter 4 A Metric for RAPidly SPinNing Black Holes . . . . . . . 109

4.1 Construction of a Kerr-like Black Hole Metric . . . . . . . . . . . . 110

4.2 Constraints on the Function $h(r, \theta) \ldots \ldots \ldots \ldots \ldots \ldots$

4.3 Metric Properties . . . . . . . . . . . . . . . . . . . . . . 119

4.3 .1 Event Horizon . . . . . . . . . . . . . . . . . . . . . . . . 119

4.3.2 Energy and Axial Angular Momentum for a Particle on a Circular Equatorial Orbit . . . . . . . . . . . . . . . . 122

4.3.3 Innermost Stable Circular Orbit and Circular Photon Orbit . 125

4.4 Conclusions . . . . . . . . . . . . . . . . . . . . 129

Chapter 5 RAY-TRACing Algorithm . . . . . . . . . . . . . . . . . . 131

5.1 A Vacuum Metric with an Arbitrary Quadrupole Moment . . . . 132

5.2 The Ray Tracing Algorithm . . . . . . . . . . . . . . . . . . 134

5.3 Relativistically Broadened Fluorescent Lines Around Quasi-Kerr Black Holes . . . . . . . . . . . . . . . . . . . . . . 140

5.4 Conclusions . . . . . . . . . . . . . . . . . . . 145

Chapter 6 Black Hole Images . . . . . . . . . . . . . . . . . . . 146

6.1 Testing the No-Hair Theorem with Black-Hole Images . . . . . . . 147

6.2 The Apparent Shape of Quasi-Kerr Black Holes . . . . . . . . . . . . 149

6.3 Photon Rings . . . . . . . . . . . . . . . . . . . . . . 153

6.4 Photon Ring Properties . . . . . . . . . . . . . . . . . . 158

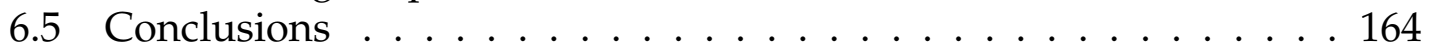

Chapter 7 Masses of Nearby Supermassive Black Holes With Very-

LONG BASELINE INTERFEROMETRY . . . . . . . . . . . . . . . . . . 167

7.1 Measuring the Photon Ring of $\operatorname{Sgr} A^{*} \ldots \ldots \ldots$ 


\section{TABLE OF CONTENTS - Continued}

7.2 Other Sources . . . . . . . . . . . . . . . . . . . 181

7.3 Discussion .......................... 187

Chapter 8 QuAsi-Periodic VARIABILITy . . . . . . . . . . . . . . . . . . 189

8.1 Dynamical Frequencies in the Quasi-Kerr Spacetime . . . . . . . . . 190

8.1.1 The Keplerian Frequency $\Omega_{\phi}$. . . . . . . . . . . . . . . . . . 192

8.1.2 The Radial and Vertical Epicyclic Frequencies . . . . . . . . 193

8.1.3 Stable Orbits in the Quasi-Kerr Spacetime . . . . . . . . . . . 196

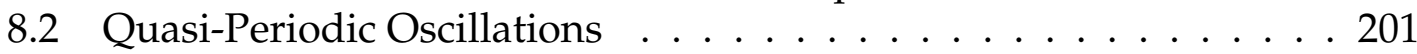

8.2.1 The Diskoseismology Model . . . . . . . . . . . . . . . 201

8.2.2 The Kinematic Resonance Model . . . . . . . . . . . . . . . . 209

8.3 Conclusions . . . . . . . . . . . . . . . . . . 212

Chapter 9 Relativistically BroAdened IRON Lines . . . . . . . . . . 214

9.1 Testing the No-Hair Theorem with Fluorescent Iron Lines . . . . . . 216

9.2 Precision Requirements for Measurements of Potential Violations of the No-Hair Theorem . . . . . . . . . . . . . . . . . . . 221

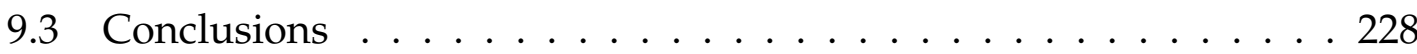

Chapter 10 Orbital Evolution of Black-Hole X-Ray Binaries . . . 230 10.1 Orbital Evolution of a Black-Hole Binary in Braneworld Gravity . . 231

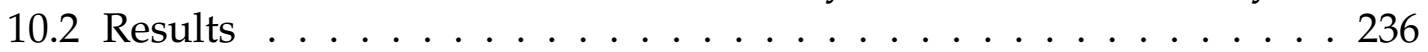

Chapter 11 Constraints on the Size of Extra Dimensions . . . . . . 241

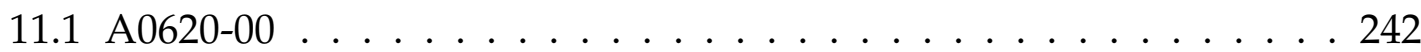

11.2 XTE J1118+480 . . . . . . . . . . . . . . . . . . . . . . . . . . . . . . . . . . . . . . . . . . . . . . . . . . . .

11.3 Results . . . . . . . . . . . . . . . . 250

11.4 Discussion ........................... 252

CHAPTER 12 SumMARY AND OUtLOOK . . . . . . . . . . . . . . . . 257

12.1 Near-Future Tests of the No-Hair Theorem . . . . . . . . . . . . . . 258

12.1.1 Modeling Continuum Disk Spectra of Black Holes in a Kerrlike Spacetime . . . . . . . . . . . . . . . . 258

12.1.2 Development of Pattern-Recognition Techniques to Image the Shadow of Sgr A* with the Event Horizon Telescope . . 259

12.1.3 Predicting X-Ray Polarization Signatures of Black Holes in a Kerr-like Spacetime . . . . . . . . . . . . . . . . . . . . . . . 260

12.1.4 Iron Line Reverberation Mapping in a Kerr-like Spacetime . 261 


\section{TABLE OF CONTENTS - Continued}

12.2 Additional Constraints on the Size of Extra Dimensions . . . . . . . 262 ApPENDix A COORdinAte TRANSFORMATIONS IN THE MN SPACETIME . 263 Appendix B Properties of A Kerr-Like Black Hole Spacetime . . . 266 Appendix C Photon Initial Conditions . . . . . . . . . . . . . . . 271

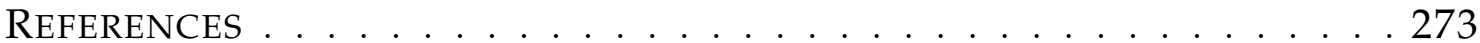




\section{LIST OF FIGURES}

1.1 Constraints on the PPN Parameter $\gamma \ldots \ldots \ldots . \ldots . \ldots . . \ldots 22$

1.2 Mass-Mass Diagram for PSR J0737-3039A/B . . . . . . . . . . . 28

1.3 Parameter Space for Quantifying the Strength of a Gravitational

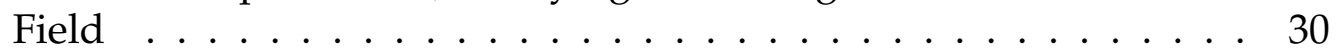

2.1 Validity of the Quasi-Kerr Metric . . . . . . . . . . . . . . 67

2.2 The Event Horizon and Static Limit in the Quasi-Kerr Metric . . . . 71

2.3 The ISCO in the Quasi-Kerr Metric . . . . . . . . . . . . . . . . 74

2.4 Equatorial Photon Trajectories in the Quasi-Kerr Metric . . . . . . . 75

2.5 Lightbending in the Quasi-Kerr Metric . . . . . . . . . . . . 76

2.6 Redshift Contours in the Quasi-Kerr Metric . . . . . . . . . . . 77

2.7 The Circular Photon Orbit in the Quasi-Kerr Metric . . . . . . . . 80

3.1 The Event Horizon in the QK Metric . . . . . . . . . . . . . . 97

3.2 The Event Horizon and Singularities in the BK Metric . . . . . . . 98

3.3 Lorentz Violations and Closed Timelike Curves in the QK Metric . 102

3.4 Lorentz Violations and Closed Timelike Curves in the BK Metric . . 103

3.5 Lorentz Violations and Closed Timelike Curves in the MN Metric . 104

3.6 Lorentz Violations and Closed Timelike Curves in the MGBK Metric 105

4.1 The Event Horizon in the JP Metric . . . . . . . . . . . . . . 116

4.2 Parameter Space in the JP Metric . . . . . . . . . . . . . . . . 121

4.3 The ISCO in the JP Metric . . . . . . . . . . . . . . . . . 122

4.4 The Circular Photon Orbit in the JP Metric . . . . . . . . . . . . . . 123

4.5 ISCO Contours in the JP Metric . . . . . . . . . . . . . . . . . 124

4.6 Event Horizon Properties . . . . . . . . . . . . . . . . . . . . . 125

4.7 The Event Horizon for Maximum Spin . . . . . . . . . . . . . 126

5.1 The Geometry of Ray Tracing . . . . . . . . . . . . . . . . . 136

5.2 Fractional Integration Error . . . . . . . . . . . . . . . . 138

5.3 Comparison of Iron Line Profiles . . . . . . . . . . . . . . . . 141

5.4 Iron Line Profiles for a Kerr Black Hole . . . . . . . . . . . . . 142

5.5 Iron Line Profiles for a Quasi-Kerr Black Hole . . . . . . . . . . . 143

6.1 Ray Tracing Geometry . . . . . . . . . . . . . . . . . . . . . 149

6.2 Images of Quasi-Kerr Black Holes . . . . . . . . . . . . . . . 151

6.3 Images of the Innermost Rings . . . . . . . . . . . . . . . 152

6.4 The Photon Ring . . . . . . . . . . . . . . . . . . 155 


\section{LIST OF FIGURES - Continued}

6.5 Spin-Dependence of the Photon Ring . . . . . . . . . . . . . . 156

6.6 Photon Ring Asymmetry . . . . . . . . . . . . . . . . . . . . . 158

6.7 Ring Displacement vs. Disk Inclination . . . . . . . . . . . . . 160

6.8 Ring Asymmetry vs. Disk Inclination . . . . . . . . . . . . . . . 161

6.9 Measurement of Black Hole Parameters . . . . . . . . . . . . . 164

7.1 Blurring due to Interstellar Scattering $\ldots \ldots \ldots \ldots$

7.2 Ring Flux Estimate . . . . . . . . . . . . . . . . . . . 175

7.3 Combined Distribution of Mass and Distance of Sgr A* . . . . . 176

7.4 Properties of Nearby Supermassive Black Holes . . . . . . . . . . . 181

8.1 The Keplerian Frequency in the Quasi-Kerr Metric . . . . . . . . . . 193

8.2 The Radial Epicyclic Frequency in the Quasi-Kerr Metric . . . . . . 195

8.3 The Lense-Thirring Frequency in the Quasi-Kerr Metric . . . . . . . 196

8.4 ISCO Contours in the Quasi-Kerr Metric . . . . . . . . . . . . . . 197

8.5 Vertical Instability of the ISCO . . . . . . . . . . . . . . . . . 198

8.6 Maximal Keplerian Frequency . . . . . . . . . . . . . . . . . . . . . . 199

8.7 Fundamental G-Mode and C-Mode Frequencies . . . . . . . . . . 203

8.8 Fundamental G-Mode and C-Mode Frequency Deviations . . . . . 204

8.9 Contours of the Fundamental G-Mode . . . . . . . . . . . . . . 205

8.10 Contours of the Fundamental C-Mode . . . . . . . . . . . . . . 206

8.11 Allowed Mass and Spin Pairs in the Diskoseismology Model . . . . 207

8.12 Black Hole Mass vs. Quadrupole Deviation in the Diskoseismology Model . . . . . . . . . . . . . . . . . . . . . . . . . 208

8.13 Mass vs. Spin in the Kinematic Resonance Model . . . . . . . . 209

8.14 Spin vs. Quadrupole Deviation in the Kinematic Resonance Model 210

9.1 Iron Line Profiles . . . . . . . . . . . . . . . . . . 217

9.2 Iron Line Degeneracies . . . . . . . . . . . . . . . . . . . . 218

9.3 Lightbending in the JP Metric . . . . . . . . . . . . . . . . . 219

9.4 Required Precision of Future X-Ray Missions . . . . . . . . . . . . . . . 221

9.5 Line Profile at Higher Inclination . . . . . . . . . . . . . . . . 223

9.6 Relative Difference of Line Profiles . . . . . . . . . . . . . . . . . 224

9.7 Contours of the Keplerian Frequency in the JP Metric . . . . . . . 225

9.8 Contours of the G-Mode and C-Mode . . . . . . . . . . . . . 226

10.1 Observed Black Hole Binaries . . . . . . . . . . . . . . 237

10.2 Rate of Change of the Orbital Period of A0620-00 . . . . . . . . . 239

10.3 Parameters for A0620-00 . . . . . . . . . . . . . . . 240 


\section{LIST OF FIGURES - Continued}

11.1 The Time of Maximal Radial Velocity of A0620-00 . . . . . . . . 243

11.2 Constraints from A0620-00 . . . . . . . . . . . . . . . . 246

11.3 Mass Dependence of Constraints . . . . . . . . . . . . . 247

11.4 Minimum Rate of Change of the Orbital Period of A0620-00 _ . . 248

11.5 Rate of Change of the Orbital Period of A0620-00 and J1118+480 . 249

11.6 Parameters of J1118+480 . . . . . . . . . . . . . . . . 250

11.7 Inferior Conjunctions of J1118+480 . . . . . . . . . . . . 252

11.8 Minimum Rate of Change of the Orbital Period of J1118+480 . . . . 253

11.9 Rate of Change of the Orbital Period of A0620-00 due to Magnetic

Braking . . . . . . . . . . . . . . . . . 255 
LIST OF TABLES

3.1 Pathologies of Kerr-like Black Hole Metrics . . . . . . . . . . . 106

7.1 Sources for VLBI Observations . . . . . . . . . . . . . . . 183

7.2 Relative Errors of Mass Measurements . . . . . . . . . . . . . . 185

10.1 Observed Properties of X-Ray Binaries . . . . . . . . . . 238

11.1 Orbital Period Measurements of A0620-00 . . . . . . . . . . . . 245

11.2 Currently Observed Orbital Periods and Times of Measurement for $\mathrm{J} 1118+480 \ldots \ldots \ldots \ldots \ldots \ldots$ 


\begin{abstract}
General relativity has been tested by many experiments, which, however, almost exclusively probe weak spacetime curvatures. In this thesis, I create two frameworks for testing general relativity in the strong-field regime with observations of black holes in the electromagnetic spectrum using current or near-future instruments.

In the first part, I design tests of the no-hair theorem, which uniquely characterizes the nature of black holes in terms of their masses and spins in general relativity and which states that these compact objects are described by the Kerr metric. I investigate a quasi-Kerr metric and construct a Kerr-like spacetime, both of which contain an independent parameter in addition to mass and spin. If the no-hair theorem is correct, then any deviation from the Kerr metric has to be zero. I show that already moderate changes of the deviation parameters in either metric lead to significant modifications of the observed signals.

First, I apply this framework to the imaging of supermassive black holes using very-long baseline interferometry. I show that the shadow of a black hole as well as the shape of a bright and narrow ring surrounding the shadow depend uniquely on its mass, spin, inclination, and the deviation parameter. I argue that the no-hair theorem can be tested with observations of the supermassive black hole Sgr A*. Second, I investigate the potential of quasi-periodic variability observed in both galactic black holes and active galactic nuclei to test the no-hair theorem in two different scenarios. Third, I show that the profiles of relativistically broadened iron lines emitted from the accretion disks of black holes imprint the signatures of deviations from the Kerr metric.
\end{abstract}


In the second part, I devise a method to test the predicted evaporation of black holes in the Randall-Sundrum model of string theory-inspired braneworld gravity through the orbital evolution of black-hole X-ray binaries and obtain constraints on the size of the extra dimension from A0620-00 and XTE J1118+480. I predict the first detection of orbital evolution in a black-hole binary. 


\section{CHAPTER 1}

\section{INTRODUCTION}

General relativity, Einstein's theory of gravity, has been tested and confirmed by a variety of different experiments ranging from Eddington's solar eclipse expedition of 1917 to modern observations of double neutron stars (Will 2006). Seldom, however, have these tests probed settings of strong spacetime curvature. To date general relativity still stands practically untested in the strong-field regime (Psaltis 2008a). In addition, none of these tests has ever accessed the highlyrelativistic environment of black holes, a unique laboratory to study some of the most extreme curvature scales in the universe. 


\subsection{Tests of the Foundations of the Gravitation Theory}

The Einstein equivalence principle (EEP) is the foundation of the gravitation theory and is comprised of the following three fundamental principles (Will 2006):

1. Weak equivalence principle (WEP)

2. Local Lorentz invariance (LLI)

3. Local Position invariance (LPI).

The WEP postulates that the trajectory of a freely falling "test" body, i.e., a body that is not affected by forces such as electromagnetism or tidal gravitational forces, is independent of its internal structure and composition. In Newtonian gravity, this statement is equivalent to the equality of the inertial and gravitational mass of such a "test" body. The LLI states that the outcome of any local non-gravitational experiment is independent of the velocity of the freely-falling reference frame in which it is performed. The LPI postulates that the outcome of such an experiment is independent of its position and the time of its performance.

It then follows from the EEP that gravitation can be described by the curvature of a spacetime (e.g., Will 1993). The only theories of gravity that are consistent with the EEP are the so-called "metric theories of gravity" (Misner et al. 1973) in which have the following properties:

1. The spacetime is endowed with a symmetric metric.

2. The trajectories of freely falling test bodies are geodesics of that metric.

3. In local freely falling reference frames, the non-gravitational laws of physics are those of special relativity. 
The three components of the EEP have been thoroughly tested by many different experiments (see Will 2006 for a review). The WEP can be tested by comparing the inertial and gravitational mass of an object. In practice, one measures the accelerations of two different bodies in an external gravitational field and determines the so-called "Eötvös ratio" named after the Hungarian physicist Loránd Eötvös (1848-1919) and given by the expression

$$
\eta \equiv 2 \frac{\left|a_{1}-a_{2}\right|}{\left|a_{1}+a_{2}\right|}
$$

The current constraint on the Eötvös ratio is $\eta<(0.3 \pm 1.8) \times 10^{-13}$ (Schlamminger et al. 2008).

In order to test the LLI, one can look for deviations of the speed of light $c$ from its predicted value $c_{0}$ in electromagnetism and measures the radio

$$
\delta \equiv\left|\frac{c_{0}^{2}}{c^{2}}-1\right| .
$$

One possible realization of such a test is to measure energy levels of atoms and nuclei trapped by laser-cooling, which would be shifted by Lorentz-violating electromagnetic interactions. At present, such shifts provide a tight constraint of $\delta<3 \times 10^{-22}$ (Lamoreaux et al. 1986).

The LPI has to be tested in two parts. Local invariance in space can be tested by measuring the gravitational redshift between two frequency standards, i.e., clocks, at different heights in a static gravitational field, which can be written by the expression

$$
\frac{\Delta \nu}{\nu} \equiv(1+\alpha) \frac{\Delta U}{c^{2}}
$$

where $\Delta U$ is the difference in the Newtonian gravitational potential between the two clocks, and the parameter $\alpha$ measures the potential deviation caused by a 
violation of the LPI. Comparing a clock in a rocket at a height of about 10,000 km to a similar clock on the ground yielded $|\alpha|<2 \times 10^{-4}$ (Vessot et al. 1980). Local invariance in time can be tested by measuring the evolution of a fundamental constant, such as the finestructure constant $\alpha_{\mathrm{EM}}$. Comparing quasar spectra to laboratory measurements established $\dot{\alpha}_{\mathrm{EM}} / \alpha_{\mathrm{EM}}=(-0.6 \pm 0.6) \times 10^{-16}$ (Srianand et al. 2004). 


\subsection{Weak-Field Tests of General Relativity}

General relativity is the most prominent example of a metric theory of gravity and has been tested throughout the solar system where spacetime curvature is small. In this regime of weak gravitational fields, the spacetime metric can be written as an expansion about the Minkowski metric $\left(\eta_{\mu \nu}=\operatorname{diag}(-1,1,1,1)\right)$ in terms of dimensionless gravitational potentials, which have a form that resembles the Newtonian potential of a given mass distribution. This is the so-called parametrized post-Newtonian framework (PPN; Will 1993).

In the solar system, the metric can be approximated as being sourced by the spherically symmetric and non-rotating sun with mass $M$, in which the planets move as test particles along geodesics. To first post-Newtonian order and in the standard coordinates $(t, x, y, z)$, the metric can, then, be written as

$$
\begin{aligned}
& g_{00}=-1+\frac{2 M}{r}-2 \beta\left(\frac{M}{r}\right)^{2}, \\
& g_{0 j}=0, \\
& g_{j k}=\left(1+2 \gamma \frac{M}{r}\right) \delta_{j k},
\end{aligned}
$$

where $r \equiv \sqrt{x^{2}+y^{2}+z^{2}}, G=c=1$, and $\beta$ and $\gamma$ are the PPN parameters. In general relativity, $\beta=\gamma=1$.

The meaning of these two PPN parameters can be inferred from the above metric. In Newtonian gravity, $g_{\mu \nu} \approx \eta_{\mu \nu}+h_{\mu \nu}$, where $h_{\mu \nu}=[-1+U(r)] \delta_{00}$ and where $U(r) \equiv 2 M / r$ is the Newtonian potential. Thus, the parameter $\beta$ measures the "nonlinearity" in the superposition law for gravity, while the parameter $\gamma$ measures the amount of space-curvature that is produced per unit rest mass. Eight additional PPN parameters exist, which vanish in general relativity (see Will 1993). 
In Schwarzschild coordinates $(t, r, \theta, \phi)$, the above metric takes the form (e.g., Hartle 2003)

$$
d s^{2}=-A(r) d t^{2}+B(r) d r^{2}+r^{2}\left(d \theta^{2}+\sin ^{2} \theta d \phi^{2}\right)
$$

where

$$
\begin{aligned}
& A(r) \equiv 1-\frac{2 M}{r}+2(\beta-\gamma)\left(\frac{M}{r}\right)^{2} \\
& B(r) \equiv 1+2 \gamma\left(\frac{M}{r}\right)
\end{aligned}
$$

\subsubsection{Light Deflection and Shapiro Time Delay}

The parameter $\gamma$ can be determined by measuring either the deflection of light or the time delay ("Shapiro delay") experienced by photons that pass by the sun closely. The deflection angle is (Hartle 2003)

$$
\delta \phi_{\text {def }}=\left(\frac{1+\gamma}{2}\right) \frac{4 M_{\odot}}{d}
$$

where $d \approx R_{\odot}$ is the impact parameter of the photon.

General relativity predicts a deflection angle of $\delta \phi_{\text {def }}=1.75^{\prime \prime}$. This deflection was first measured at optical wavelengths by Eddington and others, who confirmed this prediction with a precision of about 30\% (see Will 2006). Greater precision could be achieved at radio wavelengths using interferometry and, later, very-long baseline interferometry (VLBI). In 1995, a VLBI measurement of two quasars that were passing behind the sun yielded $(1+\gamma) / 2=0.9996 \pm 0.0017$ (Lebach et al. 1995). A combination of many VLBI observations of different radio sources yielded $(1+\gamma) / 2=0.99992 \pm 0.00023$ (Shapiro et al. 2004). A similar result with a slightly lower precision was obtained from observations of the optical satellite Hipparcos (Froeschlé, Mignard, \& Arenou 1997). The various measurements are summarized in Figure 1.1. 


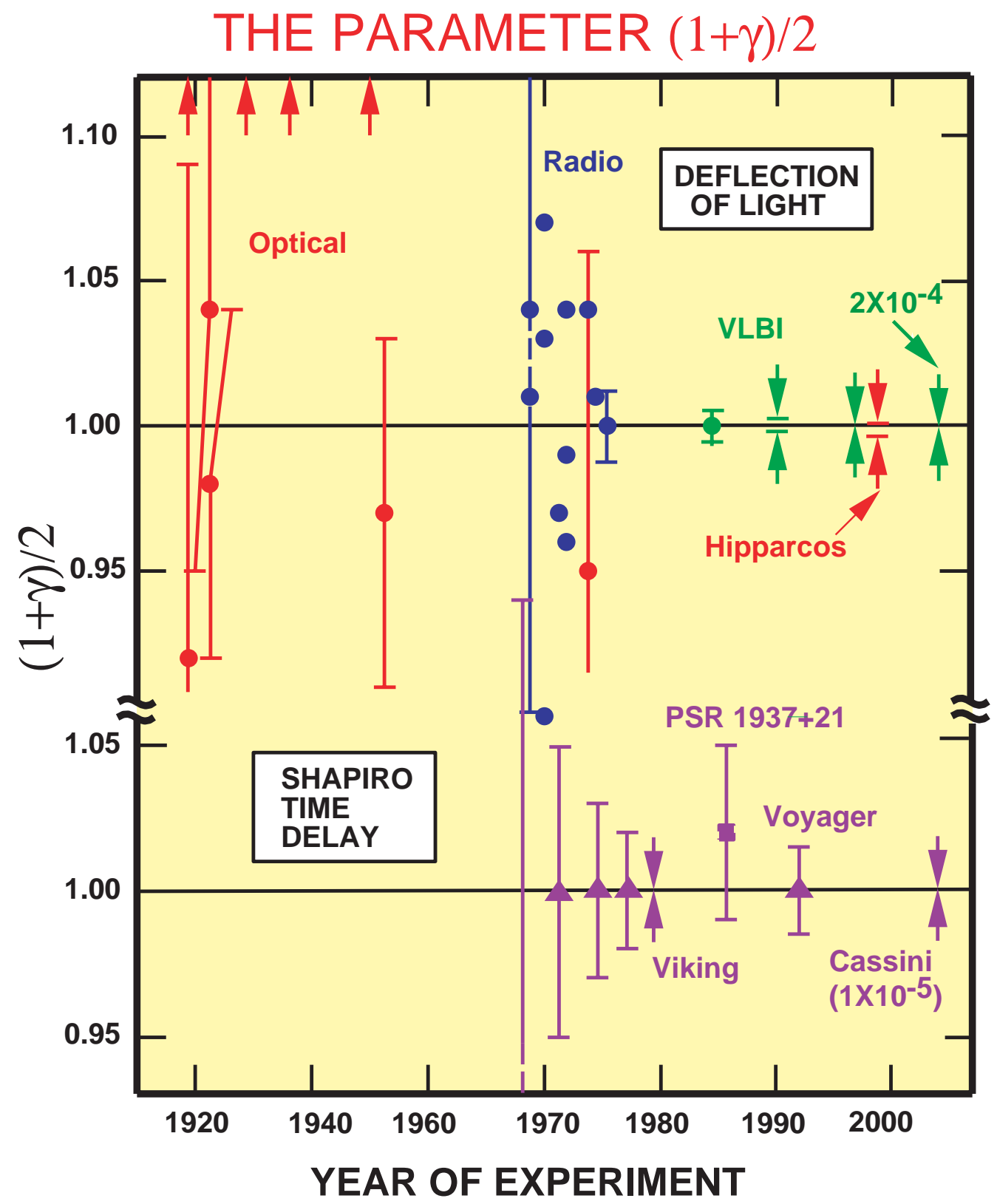

Figure 1.1 Measurements of the coefficient $(1+\gamma) / 2$ from light deflection and time delay measurements. Its value in general relativity is unity. The arrows at the top denote anomalously large values from early eclipse expeditions. The Shapiro time-delay measurements using the Cassini spacecraft yielded an agreement with general relativity to $10^{-3}$ percent, and VLBI light deflection measurements have reached 0.02 percent. Hipparcos denotes the optical astrometry satellite, which reached 0.1 percent (Will 2006). 
The Shapiro delay of a photon sent past the sun to a planet or satellite and back to Earth is given by the expression (Will 2006; Hartle 2003)

$$
\Delta t_{\mathrm{excess}}=4 M_{\odot} \frac{1+\gamma}{2}\left[\ln \left(\frac{4 r_{\oplus} r_{R}}{d^{2}}\right)+1\right] \approx \frac{1+\gamma}{2}\left(240-20 \ln \frac{d^{2}}{\tilde{r}_{R}}\right) \mu \mathrm{s},
$$

where $r_{\oplus}$ and $r_{R}$ are the distances of the Earth and the responder from the sun, respectively, and $\tilde{r}_{R}$ is equal to $r_{R}$ expressed in astronomical units.

Several measurements of the Shapiro delay have been performed, which used either radar signals sent to Mercury, Venus, and satellites such as Voyager 2, the Viking Mars landers and orbiters, and Cassini or the radio emission from the pulsar PSR 1937+21 (Will 2006). The Viking experiment in 1976 achieved a confirmation of the value of the parameter $\gamma$ in general relativity to within 0.1 percent (Reasenberg et al. 1979). The best result was obtained from the Cassini satellite on its way to Saturn in 2003 with a measurement of $\gamma-1=(2.1 \pm 2.3) \times 10^{-5}$ (Bertotti, Iess, \& Tortora 2003). See Figure 1.1 for a compilation of these measurements.

\subsubsection{The Perihelion Precession of Mercury}

The parameter $\beta$ can be measured from observations of the perihelion precession of Mercury's orbit. The general-relativistic prediction of this precession is $\Delta \phi_{\text {prec }}=42.98^{\prime \prime} /$ century (e.g., Hartle 2003). In terms of the PPN parameters, the advance per orbit is given by the expression (Will 2006)

$$
\delta \phi_{\text {prec }}=\frac{6 \pi M_{\odot}}{p}\left[\frac{1}{3}(2+2 \gamma-\beta)+\frac{J_{2} R_{\odot}}{2 M_{\odot} p}\right],
$$

which includes the Newtonian contribution from a possible (dimensionless) solar quadrupole moment $J_{2}$ and where $p \equiv a_{1}\left(1-e^{2}\right)$ is the semi-latus rectum of the orbit with a semi-major axes $a_{1}$ and eccentricity $e$. From helioseismological observations of oscillation modes of the sun, Mecheri et al. (2004) find $J_{2}=(2.2 \pm$ 
$0.1) \times 10^{-7}$. Substituting the standard orbital elements in Equation (1.12), the perihelion shift per century is given by the expression

$$
\Delta \phi_{\text {prec }}=42.98^{\prime \prime}\left[\frac{1}{3}(2+2 \gamma-\beta)+3 \times 10^{-4} \frac{J_{2}}{10^{-7}}\right] .
$$

Various measurements of the perihelion shift have been obtained (see, e.g., Shapiro 1990). The effect of the solar oblateness is smaller than the observational error of the perihelion shift of these measurements, which, then, yield the bound (Will 2006)

$$
|2 \gamma-\beta-1|<3 \times 10^{-3}
$$

Nordtvedt (1968) showed that the WEP can be violated in the PPN formalism leading to an inequality of the inertial $\left(m_{I}\right)$ and gravitational $\left(m_{G}\right)$ masses of a body of the form

$$
\frac{m_{G}}{m_{I}}=1-\eta_{N} \frac{E_{G}}{m_{I}}
$$

where $-E_{G}$ is the gravitational self-energy of the body and where

$$
\eta_{N}=4 \beta-\gamma-3
$$

This effect is absent in general relativity $\left(\eta_{N}=0\right)$ and does not violate the results of laboratory Eötvs̈ experiments, because in the case of laboratory-sized masses $E_{G} / m_{I} \sim 10^{-27}$ (Will 2006). This effect, on the other hand, can be significant for celestial bodies $\left(E_{G} / m_{I} \sim 10^{-10}\right.$ for the moon; Will 2006). However, precision measurements of the Earth-moon distance over time using the reflection of laser signals from the moon (Lunar Laser Ranging; LLR) with accuracies of the order of 50 ps $(1 \mathrm{~cm})$ found no evidence for this effect (Williams, Turyshev, \& Murphy 2004). In combination with the constraints on the parameter $\gamma$ from the Cassini 
experiment, this measurement implies (Williams, Turyshev, \& Boggs 2004)

$$
\beta-1=(1.2 \pm 1.1) \times 10^{-4} .
$$

\subsubsection{Gravitomagnetism}

Another important test of post-Newtonian gravity is the search for "gravitomagnetism," the effect of a moving or rotating body to produce a contribution to the gravitational field in analogy to the magnetic field of a moving charge or a magnetic dipole. Such an effect is expected, because in theories of gravity like general relativity the gravitational field is sourced by mass-energy and not just by mass alone. In the PPN formalism with coordinates $(t, x, y, z)$, this effect can be expressed in terms of the metric elements

$$
g_{0 i}=-\frac{1}{2}(4 \gamma+4) V_{i} .
$$

For a rotating body with angular momentum $J, \mathbf{V}=-\frac{1}{2} \mathbf{x} \times \mathbf{J} / \mathbf{r}^{3}$. The result is the "dragging of inertial frames" around the body, which is also called the LenseThirring effect. This effect leads to a precession of the spin $\mathbf{S}$ of a gyroscope placed in that field, which is given by the expression

$$
\frac{d \mathbf{S}}{d \tau}=\boldsymbol{\Omega}_{\mathrm{LT}} \times \mathbf{S},
$$

where

$$
\boldsymbol{\Omega}_{\mathrm{LT}} \equiv-\frac{\mathbf{1}}{\mathbf{2}}(\mathbf{1}+\gamma) \frac{\mathbf{J}-\mathbf{3} \mathbf{n}(\mathbf{n} \cdot \mathbf{J})}{\mathbf{r}^{\mathbf{3}}}
$$

is the Lense-Thirring frequency and $\mathbf{n}$ is the unit radial vector.

Such a gyroscope moving with velocity $\mathbf{v}$ also experiences a geodetic precession of its spin axis caused by the gradient of the Newtonian gravitational potential according to

$$
\frac{d \mathbf{S}}{d \tau}=\boldsymbol{\Omega}_{\mathrm{G}} \times \mathbf{S},
$$


where

$$
\boldsymbol{\Omega}_{\mathrm{G}} \equiv\left(\gamma+\frac{1}{2}\right) \mathbf{v} \times \nabla \mathbf{U}
$$

The Relativistic Gyroscope Experiment (Gravity Probe B) recently completed a space mission to measure the Lense-Thirring and the geodetic precessions of a gyroscope in an orbit around the Earth. The angular precessions predicted by general relativity were $-6606.1 \mathrm{mas} / \mathrm{yr}$ and $-39.2 \mathrm{mas} / \mathrm{yr}$, respectively, and the measured precessions were $-6601.8 \pm 18.3 \mathrm{mas} / \mathrm{yr}$ and $-37.2 \pm 7.2 \mathrm{mas} / \mathrm{yr}$ confirming the predictions of general relativity to within $0.28 \%$ and $19 \%$, respectively (Everitt et al. 2011).

\subsubsection{Timing Observations of Double Pulsars}

Several pairs of pulsars orbiting around their respective centers of mass have been discovered to date (Lorimer 2005). Among them are the "Hulse-Taylor" pulsar PSR B1913+16 (Hulse \& Taylor 1975) and the double pulsar PSR J07373039A/B (Burgay et al. 2003; Lyne et al. 2004). Due to the compactness of such systems (semi-major axis $\sim 1 R_{\odot}$ ) with orbital periods of only several hours and the high precision of measurements of their pulse profiles, timing observations of double pulsars can provide a very good testing ground for weak-field general relativity (Stairs 2003).

Double pulsars have three groups of parameters: non-orbital parameters such as their pulse periods, the rates of change of these periods, and their positions in the sky; five "Keplerian" parameters such as the eccentricity $e$, the orbital period $P_{b}$, and the semi-major axis; as well as five "post-Keplerian" parameters such as the mean rate of periastron advance $\langle\dot{\omega}\rangle$, the redshift/time dilation $\gamma^{\prime}$ of the emitted radio pulses, and the orbital period derivative $\dot{P}_{b}$. Assuming general rel- 
ativity, the post-Keplerian parameters can be expressed in terms of the Keplerian parameters and the two unknown pulsar masses according to the expressions (Damour \& Taylor 1992)

$$
\begin{aligned}
\langle\dot{\omega}\rangle & =6 \pi f_{b}\left(2 \pi m f_{b}\right)^{2 / 3}\left(1-e^{2}\right)^{-1} \\
\gamma^{\prime} & =e\left(2 \pi f_{b}\right)^{-1}\left(2 \pi m f_{b}\right)^{2 / 3} \frac{m_{2}}{m}\left(1+\frac{m_{2}}{m}\right), \\
\dot{P}_{b} & =-\frac{192 \pi}{5}\left(2 \pi \mathcal{M} f_{b}\right)^{5 / 3} F(e), \\
s & =\sin i \\
r & =m_{2},
\end{aligned}
$$

where $r$ and $s$ are the "range" and "shape" of the Shapiro delay, $i$ is the inclination of the orbital plane with respect to the observer's line of sight, $m_{1}$ and $m_{2}$ are the pulsar masses, $m \equiv m_{1}+m_{2}, f_{b} \equiv 1 / P_{b}$ and

$$
\begin{aligned}
\mathcal{M} & \equiv \frac{G M_{\odot}}{c^{3}} \frac{\left(m_{1} m_{2}\right)^{3 / 5}}{\left(m_{1}+m_{2}\right)^{1 / 5}} \\
F(e) & \equiv\left(1+\frac{73}{24} e^{2}+\frac{37}{96} e^{4}\right)\left(1-e^{2}\right)^{-7 / 2} .
\end{aligned}
$$

A measurement of these three parameters provides three constraints on the two pulsar masses. These constraints, then, have to provide a consistent solution of the masses within measurement uncertainties. An analysis of the data of the Hulse-Taylor double pulsar yields (Will 2006) $m_{1}=1.4414 \pm 0.0002 M_{\odot}$ and $m_{2}=1.3867 \pm 0.0002 M_{\odot}$, while measurements of the parameters of PSR J07373039A/B yield (Kramer et al. 2006) $1.3381 \pm 0.0007 M_{\odot}$ and $m_{2}=1.2489 \pm 0.0007 M_{\odot}$ confirming general relativity at the $0.01 \%$ level. Figure 1.2 (the "mass-mass diagram" of a double pulsar system) shows the three post-Keplerian parameters as a function of the two pulsar masses. 


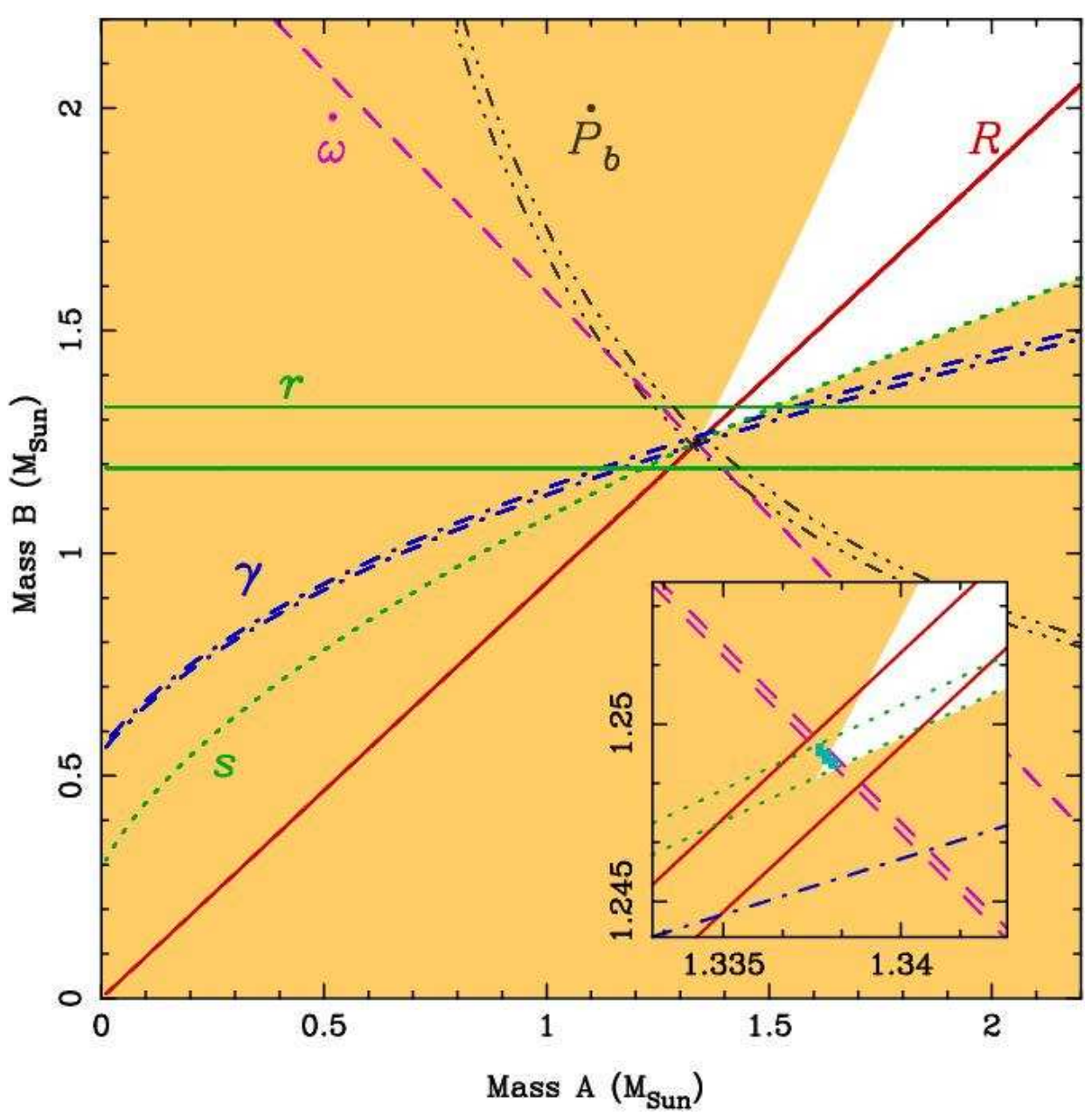

Figure 1.2 Graphical summary of tests of the general-relativistic parameters. Constraints on the masses of the two pulsars (A and B) in the PSR J0737-3039A/B binary system are shown; the inset is an expanded view of the region of principal interest. Shaded regions are forbidden by the individual mass functions of $\mathrm{A}$ and $\mathrm{B}$ because $\sin i \leq 1$. Other constraining parameters are shown as pairs of lines, where the separation of the lines indicates the measurement uncertainty. For the diagonal pair of lines labeled as $R$, representing the mass ratio derived from the measured semimajor axes of the A and B orbits, the measurement precision is so good that the line separation becomes apparent only in the inset. The other constraints shown are based on the measured post-Keplerian parameters interpreted within the framework of general relativity. The intersection of all line pairs is consistent with a single point that corresponds to the masses of A and B. The current uncertainties in the observed parameters determine the size of this intersection area, which is marked in blue and reflects the achieved precision of this test of general relativity and the mass determination for A and B (Kramer et al. 2006). 


\subsection{Testing General Relativity in the Strong-Field Regime}

The tests described so far place tight limits on the properties of theories of gravity in the weak-field regime and leave little room for deviations from general relativity. In the strong-field regime, however, two theories of gravity can differ significantly in their predictions, even though both of them satisfy the current experimental constraints. It is, therefore, incumbent to test general relativity in the strong-field regime.

For an object of mass $M$, it is instructive to distinguish strong and weak gravitational fields in terms of the gravitational potential

$$
\varepsilon \equiv \frac{G M}{r c^{2}}
$$

and the spacetime curvature

$$
\xi \equiv \frac{G M}{r^{3} c^{2}},
$$

where the coordinate $r$ measures the radial distance from the source (Psaltis 2008a). While these quantities are not precise, because the gravitational potential $\varepsilon$ is only valid in Newtonian gravity and because curvature is described in terms of a tensor, the Riemann tensor, they allow for an intuitive characterization of strong and weak fields using dimensional arguments. In the solar system, where tests of general relativity to date have been performed almost exclusively, the "strongest" gravitational potential and curvature are, respectively,

$$
\begin{aligned}
& \frac{G M_{\odot}}{R_{\odot} c^{2}} \simeq 2 \times 10^{-6} \\
& \frac{G M_{\odot}}{R_{\odot}^{3} c^{2}} \simeq 4 \times 10^{-28} \mathrm{~cm}^{-2} .
\end{aligned}
$$

In contrast, in the vicinity of neutron stars and black holes, these quantities can be as large as $\varepsilon \sim 1$ and $\xi \sim 10^{-13} \mathrm{~cm}^{-2}$ (Psaltis 2008). 


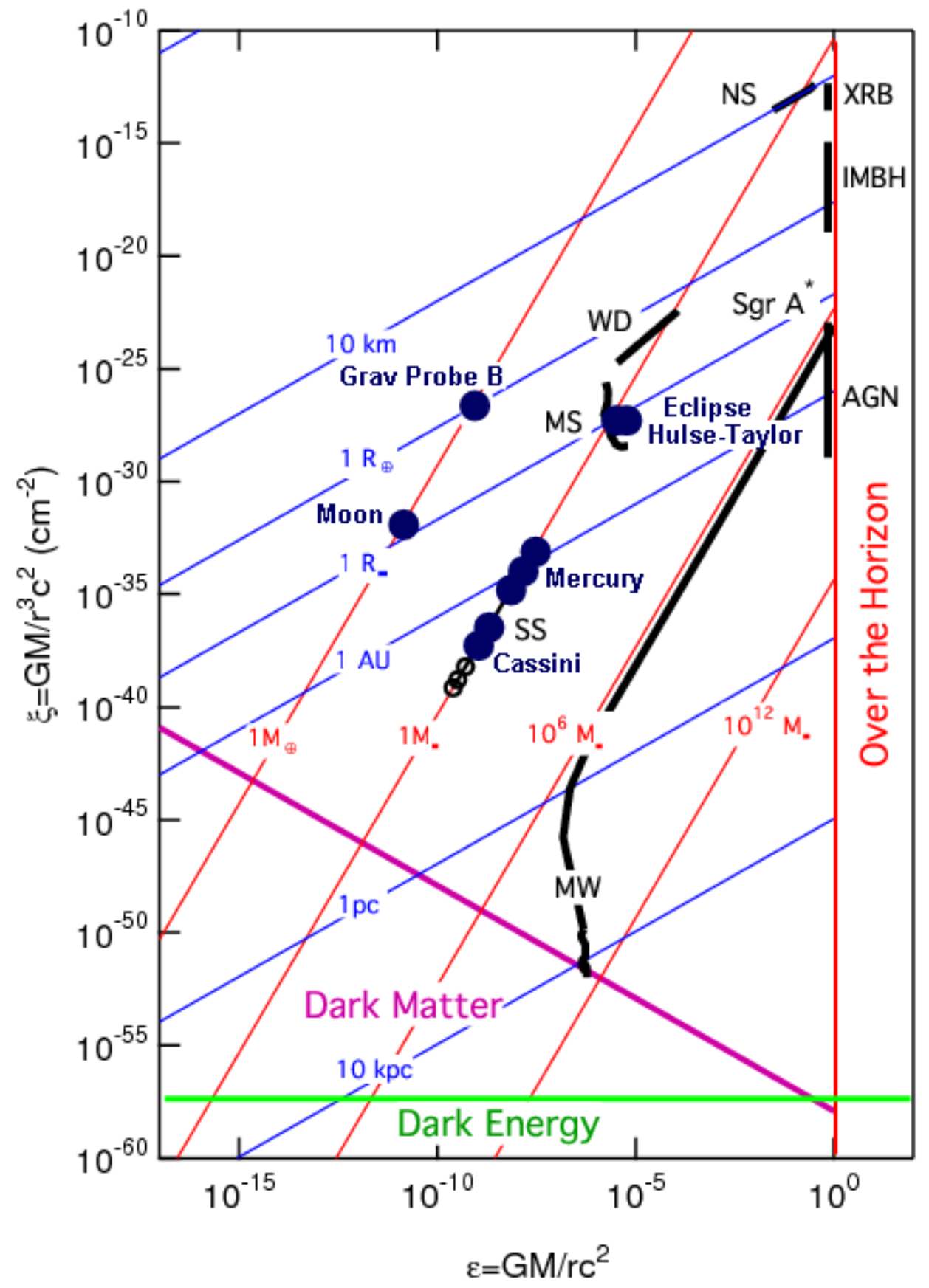

Figure 1.3 A parameter space for quantifying the strength of a gravitational field. The $x$-axis measures the potential $\varepsilon \equiv G M / c^{3}$ and the $y$-axis the spacetime curvature $\xi \equiv G M / r^{3} c^{2}$ of the gravitational field at a radius $r$ away from the central object of mass $M$. The various curves, points, and legends are explained in the text. Regions of the parameter space that have been probed by experiment are labeled in dark blue (after Psaltis 2008a). 
The gravitational potential $\varepsilon$ and the curvature $\xi$ define a parameter space, where the gravitational field strengths probed by different tests of gravity can be quantified (see Figure 1.3). Only a small fraction of this space has been accessed by current experiments. The gravitational potential has an upper bound of $\varepsilon \simeq 1$ corresponding to the event horizon of a black hole and denoted by the vertical red line.

The presence of dark energy in the form of a cosmological constant affects gravitational experiments only if

$$
\xi \leq \frac{3 G \Omega_{\Lambda} H_{0}^{2}}{8 \pi c^{2}}
$$

where $\Omega_{\Lambda}$ is the current dark energy density in units of the critical density and $H_{0}$ is the current value of the Hubble constant (i.e., below the green line in Figure 1.3). Dark matter typically becomes important if the acceleration of a particle drops below the so-called MOND acceleration scale $a_{0} \simeq 10^{-8} \mathrm{~cm} \mathrm{~s}^{-2}$, which corresponds to values of the curvature

$$
\xi \leq\left(\frac{a_{0}}{c^{2}}\right)^{2} \frac{1}{\varepsilon} \simeq\left(\frac{H_{0}}{c}\right)^{2} \frac{1}{\varepsilon} .
$$

This part of the parameter space is delineated by the purple line in Figure 1.3.

In the opposite extreme of very strong gravitational fields (not shown in Figure 1.3), classical theories of gravity are expected to break down when quantum effects become important. This occurs at distances that are comparable to the Compton wavelength $\lambda_{C} \equiv h / M c$, where $h$ is Planck's constant, corresponding to values of the curvature

$$
\xi \geq \frac{1}{L_{P}^{2}} \varepsilon^{2},
$$

where $L_{P} \equiv\left(G h / c^{3}\right)^{1 / 2} \simeq 4 \times 10^{-33} \mathrm{~cm}$ is the Planck length. The various labels in Figure 1.3 denote neutron stars (NS), black holes in X-ray binaries (XRB), 
intermediate-mass black holes (IMBH), active galactic nuclei (AGN), white dwarfs (WD), main-sequence stars (MS), planets in our solar system (SS), the supermassive black hole at the center of the Milky Way (Sgr A*), and studies of the rotational curves of the Milky Way and other galaxies (MW). Regions of the parameter space that have been probed by experiment are labeled in dark blue.

To date, there have been only a handful of strong-field tests of general relativity in the context of either neutron stars (see DeDeo \& Psaltis 2003; Psaltis 2008a) or black holes (Psaltis 2007). All of these, however, tested only very specific predictions of selected modified theories of gravity such as Brans-Dicke gravity (Brans \& Dicke 1961) or the second-order scalar-tensor theory by Damour \& Esposito-Farèse $(1993,1996)$ and did not search for generic deviations from general relativity.

Such tests are a priori limited by the fact that observations are interpreted within the narrow confines of a particular theory. Our lack of knowledge of a quantum theory of gravity as part of a grant unified theory of all fundamental interactions forces us to consider many different alternatives to general relativity. It is, therefore, advisable to search for deviations from general relativity using a broader setting that encompasses as many modified theories of gravity as possible (Psaltis 2009). In this phenomenological approach, stars and compact objects have properties that differ from those in general relativity. These deviations can be expressed in terms of free parameters, which can, in principle, be determined by observation. The confirmation or exclusion of the predictions of different gravity theories can then lead to a greater understanding of the fundamental theory of gravity (Psaltis 2009). In this thesis, I focus on two essential properties of general relativity, the no-hair theorem and the assumption that spacetime is 
four-dimensional. 


\subsection{Testing the No-Hair Theorem}

According to the no-hair theorem, black holes in general relativity are uniquely characterized by only three parameters: their masses, spins, and charges (Israel 1967, 1968; Carter 1971, 1973; Hawking 1972; Robinson 1975; Mazur 1982). The spacetimes of such black holes are described by the Kerr-Newman metric (Newman et al. 1965), which reduces to the Kerr metric (Kerr 1963) in the case of uncharged black holes. This theorem relies on the cosmic censorship conjecture (Penrose 1969) as well as on the physically reasonable assumption that the exterior metric is free of closed timelike loops. If these requirements are met, then all astrophysical black holes should be described by the Kerr metric.

Black holes are commonly believed to be the final states of the evolution of sufficiently massive stars at the end of their lifecycle. The gravitational collapse of such stars leads to the formation of a black hole (Oppenheimer \& Snyder 1939; Penrose 1965), and any residual signature of the progenitor other than its mass and spin is radiated away by gravitational radiation (Price 1972a, b). This scenario provides an astrophysical mechanism with which Kerr black holes can be generated. Almost all nearby galaxies harbor dark objects of high mass and compactness at their center (Kormendy \& Richstone 1995) including our own galaxy (Ghez et al. 2008; Gillessen et al. 2009) providing strong evidence that black holes are realized in nature. In addition, the measurement of the orbital parameters of many galactic binaries supports the claim that they contain stellar-mass black holes (e.g., McClintock \& Remillard 2006).

Despite the large amount of circumstantial evidence, there has been no direct proof, so far, for the existence of an actual event horizon. An event horizon, one of the most striking predictions of general relativity, is a virtual boundary that 
causally disconnects the interior of a black hole from the exterior universe. The presence of an event horizon in black-hole candidates has only been inferred indirectly, either from the properties of advection dominated accretion flows or from the lack of observations of Type I X-ray bursts (Narayan, Garcia, \& McClintock 1997, 2001; Narayan \& Heyl 2002; McClinock, Narayan, \& Rybicki 2004; Broderick et al. 2009b; see discussion in Psaltis 2006).

\subsubsection{Violations of the No-Hair Theorem}

The alternative hypothesis that these dark compact objects are not described by the Kerr metric but perhaps by a solution of the Einstein equations with a naked singularity (e.g., Manko \& Novikov 1992) and, therefore, violate the no-hair theorem, is still possible within general relativity. Alternatively, these dark objects might be stable stellar configurations consisting of exotic fields (e.g., boson stars, Friedberg, Lee, \& Pang 1987; gravastars, Mazur \& Mottola 2001; black stars, Barceló et al. 2008). Finally, the fundamental theory of gravity may be different from general relativity in the strong-field regime, and the vacuum black-hole solution might not be described by the Kerr metric at all (e.g., Yunes \& Pretorius 2009; Sopuerta \& Yunes 2009; c.f. Psaltis et al. 2008). As a result, testing the no-hair theorem allows us not only to verify the identification of dark compact objects in the universe with Kerr black holes but to test the strong-field predictions of general relativity, as well.

The exterior spacetime of a black-hole candidate is usually best described in terms of its multipole moments. The mass and the spin of a black hole can be identified as the first two such moments. As a consequence of the no-hair theorem, mass and spin already specify all higher multipole moments of a black-hole spacetime completely. Therefore, measuring three independent multipole mo- 
ments of the spacetime around a black hole suffices to test the no-hair theorem (Ryan 1995).

Several tests of the no-hair theorem have been suggested using observations of gravitational waves from extreme mass-ratio inspirals (Ryan 1995, 1997a, b; Barack \& Cutler 2004, 2007; Collins \& Hughes 2004; Glampedakis \& Babak 2006; Gair et al. 2008; Li \& Lovelace 2008; Apostolatos et al. 2009; Vigeland \& Hughes 2010; Vigeland et al. 2011; Gair \& Yunes 2011; Rodriguez et al. 2012) and from electromagnetic observations of stars orbiting around Sgr A* (Will 2008; Merritt et al. 2010; Sadeghian \& Will 2011), of pulsar black-hole binaries (Wex \& Kopeikin 1999; Liu et al. 2012), as well as of the quasar OJ287 (Valtonen et al. 2011). While tests in the gravitational-wave spectrum probe the strong-field nature of black holes, these electromagnetic tests rely on observations of companion stars or compact objects at comparatively large distances and, therefore, can only probe the spacetimes of black holes where their gravitational filed is weak.

In the first part of this thesis, I develop another class of observational tests of the no-hair theorem in the electromagnetic spectrum, which aim to probe the spacetimes of black holes in the strong-field regime using the emission from their accretion flows. In contrast to weak-field tests of the no-hair theorem, where a parameterized post-Newtonian approach (e.g., Will 1993) is sufficient, strongfield tests require a careful modeling of the spacetime by a parametric deviation from the Kerr metric. The objects they describe have spacetimes that deviate slightly from the Kerr metric, and observables can be studied in terms of one (or more) free parameters. All of these metrics contain the Kerr metric as the special case when the deviations are dialed to zero.

This approach allows for a two-fold test: If the central object is indeed a black 
hole, a deviation from the Kerr metric must be zero. If, however, the deviation is measured to be nonzero, then it either has to be a different type of object or the no-hair theorem is violated and general relativity itself breaks down in the strongfield regime very close to the black hole (c.f., Collins \& Hughes 2004; Hughes 2006).

In Chapter 2, I formulate these tests in terms of a quasi-Kerr metric (Glampedakis \& Babak 2006) that incorporates an independent quadrupole moment. This metric is a perturbation of the Kerr metric and a vacuum solution of the Einstein field equations up to the quadrupole order and can, therefore, only be used to describe moderately spinning black holes for which higher-order moments can be neglected. I analyze in detail the properties of this metric that are critical for observational tests of the no-hair theorem in the electromagnetic spectrum.

In addition to the quasi-Kerr spacetime, other parametric deviations from the Kerr metric have been developed (e.g., Manko \& Novikov 1992; Collins \& Hughes 2004; Vigeland \& Hughes 2010; Vigeland et al. 2011). Because of the no-hair theorem, all parametric deviations of the Kerr metric within general relativity have to violate at least one of the prerequisites of this theorem. Consequently, these spacetimes contain either singularities or regions with closed timelike curves outside of the event horizon. The degree to which these pathologies affect different proposed tests of the no-hair theorem depends on the intended application. For all the currently proposed metrics that deviate from the Kerr solution, pathologies appear very close to the corresponding Kerr event horizon. As a result, they do not hamper tests of the no-hair theorem that involve the orbits of objects at large distances from the horizons, as is the case, e.g., for tests with extreme mass-ratio inspirals (Barack \& Cutler 2007) or observations of stars and 
pulsars around black holes (Will 2008; Wex \& Kopeikin 1999). These pathologies, however, become prohibitive in cases of tests that involve observations of the inner accretion flows of black holes.

For the latter tests and for moderately spinning black holes, the singularities or closed time-like loops appear far inside the location of the photon orbit and the location of the innermost stable circular orbit (ISCO), both of which dominate the observational characteristics of black holes. These pathologies can, therefore, be handled by imposing an artificial cutoff with an inflow boundary condition at some radius in the exterior spacetime, between the location of the pathologies and the location of the photon orbit or ISCO. For rapidly spinning black holes, however, the radius of the ISCO becomes comparable to the radius of the horizon and imposing such an artificial cut-off is no longer possible. This limits the applicability of current parametric deviations of the Kerr metric for several observational tests of the no-hair theorem in the electromagnetic spectrum to only moderately spinning black holes.

In Chapter 3, I analyze the properties of several parametric deviations from the Kerr spacetime. I aim to identify the manner in which these four metrics violate the no-hair theorem and determine their respective ranges of applicability for observational tests of the no-hair theorem as a function of spin and the strength of the deviation.

Performing tests of the no-hair theorem with observations of phenomena that occur in the vicinity of the circular photon orbit or the ISCO around a black holes requires that I use a metric that is free of such pathologies for arbitrary values of the spin. However, finding a metric of this kind is a very difficult task. Introducing small parametric deviations to individual elements of the metric in an 
arbitrary manner routinely leads to the pathologies discussed above. In order for such a spacetime to describe a black hole, it can no longer be a solution of the Einstein equations, because otherwise it would render the no-hair theorem false. To date, however, black hole metrics for theories that obey the Einstein equivalence principle (Will 1993) are only known for static black holes (e.g., Yunes \& Stein 2011; Dai \& Stojkovic 2010), for slowly rotating black holes with parity violations (Yunes \& Pretorius 2009), or in Einstein-Dilaton-Gauss-Bonnet gravity (Pani \& Cardoso 2009).

In Chapter 4, I construct such a Kerr-like black hole metric which suffers from no pathologies up to the maximum value of the spin and which contains a set of parameters that measure potential deviations from the Kerr metric in the strongfield regime. In order to achieve this in a regular manner, I start by introducing a parametric deviation to the Schwarzschild metric, following Yunes \& Stein (2011). I then apply the Newman-Janis algorithm (Newman \& Janis 1965; Drake \& Szekeres 2000), as in Vigeland \& Hughes (2010), in order to generate a metric for a spinning black hole.

I take special care to retain several properties that make the Kerr metric unique in performing ray-tracing calculations in general relativity. My metric shares the same non-zero metric elements with the Kerr solution, which allows for a straightforward implementation for calculations of ray tracing with existing geodesic algorithms and an intuitive interpretation of observables. I likewise obtain constraints for some of the parameters of my metric from observational limits on modifications of general relativity in the weak-field regime as well as from the requirements of asymptotic flatness.

For the particular case of only one deviation parameter, I show that my metric 
is regular everywhere outside the horizon for the entire range of allowable spins up to a maximum value, which depends on the deviation. It can, therefore, be used to study astrophysical phenomena arbitrarily close to the event horizon and to test the no-hair theorem in the electromagnetic spectrum even with rapidly spinning black holes. I also derive expressions for the energy and angular momentum of a particle on a circular equatorial orbit around the central black hole and compute the locations of the ISCO and the circular photon orbit as a function of spin and the deviation parameter.

\subsubsection{Prediction of Observational Signatures of Violations of the No-Hair Theo- rem}

Observables in parametric deviations from the Kerr metric can oftentimes only be analyzed in terms of numerical techniques. The Kerr metric is of Petrov-type $\mathrm{D}$ and, therefore, the Carter constant is an integral of motion along the trajectories of photons (Carter 1968). The presence of this integral of motion, in addition to the conservation of energy and angular momentum, allows one to use first-order differential equations to solve for the photon trajectories, which produce the observed signals. This improves the accuracy of the calculation and increases its speed (e.g., Rauch \& Blandford 1994; Dexter \& Agol 2009; see, however, Broderick 2006; Dolence et al. 2009 for different approaches).

Introducing any deviation from the Kerr metric does usually not preserve its Petrov-type D character and the Carter constant is no longer conserved along geodesics (see, e.g., Glampedakis \& Babak 2006; Gair, Li, \& Mandel 2008). As a result, ray tracing in a non-Kerr metric cannot be performed entirely using integrals of motions but requires integrating the second-order differential equations for individual geodesics. 
In Chapter 5, I discuss a new ray-tracing algorithm for calculating the observational appearance of spinning compact objects for arbitrary Kerr-like spacetimes. In the algorithm, I integrate two first-order differential equations that arise from integrals of motion as well as two second-order differential equations for two components of the geodesic equations in order to compute the trajectories of photons in these spacetimes. I also use a third integral of motion related to the norm of the photon 4-momenta in order to monitor the accuracy of the calculations.

I, then, make use of this algorithm to design tests of the no-hair theorem in three different settings. First, I focus on imaging observations of accreting black holes at (sub-)mm wavelengths using very-long baseline interferometry (VLBI). Such observations promise to enable unprecedented views of the vicinities of black-hole horizons. Recent VLBI-observations along only three baselines resolved Sgr A*, the black hole in the center of the Milky Way, on a scale comparable to its event horizon and provided evidence for sub-horizon scale structures (Doeleman et al. 2008) as well as for the presence of an event horizon (Broderick, Loeb, \& Narayan 2009). Far greater resolution can be achieved by the Event Horizon Telescope, a planned global array of (sub-)mm telescopes (Doeleman et al. 2009a, b; Fish et al. 2009).

In Chapter 6, I study the properties of the images of compact objects that violate the no-hair theorem using the quasi-Kerr formalism. I calculate numerically the mapping between locations in the vicinity of a black hole and positions in the observer's sky using the mass, spin, and quadrupole moment of the spacetime as independent parameters. I investigate the impact of varying the quadrupole moment on the properties of this mapping and show that the images of the accretion flows around compact objects that violate the no-hair theorem are expected 
to have prolate or oblate geometries.

Measuring the spacetime moments from the images of an accretion flow will be, of course, very model dependent and limited by our lack of understanding of the intrinsic geometry of the flow itself. For example, prolate images of the inner accretion may be the result of resolving the formation region of a jet and not of a violation of the no-hair theorem (see, e.g., Broderick \& Loeb 2009). Moreover, a measurement of the spin from an image of the shadow alone is difficult (e.g., Falcke et al. 2000; Takahashi 2004) and might require complementary observations such as a multiwavelength study of polarization (Broderick \& Loeb 2006; see also Schnittman \& Krolik 2009, 2010).

Additionally, accretion flows are very turbulent and variable at timescales much shorter than the rotation period of the Earth, which sets the characteristic integration time for an interferometric imaging observation. If this time variability is produced by a highly coherent, orbiting inhomogeneity in the accretion flow, it may allow measuring the properties of the compact object via nonimaging techniques (Doeleman et al. 2009). The variability, however, will limit and may prohibit altogether the ability of obtaining a clean image of the accretion flow.

The images of optically thin accretion flows around black holes, however, reveal a characteristic bright ring at the projected radius of the circular photon orbit along null geodesics (Beckwith \& Done 2005) with properties that remain constant even as the underlying accretion flows are highly variable. This bright ring is the result of the light rays that orbit around the black hole many times before they reach the distant observer, and, therefore, have a much larger path length through the optically thin accretion flow. These photons can make a sig- 
nificant contribution to the total disk emission and produce higher-order images (Cunningham 1976; Laor, Netzer, \& Piran 1990; Viergutz 1993; Bao, Hadrava, \& Østgaard 1994; Čadež, Fanton, \& Calvani 1998; Agol \& Krolik 2000; Beckwith \& Done 2005).

I use the quasi-Kerr formalism to show that the bright emission ring is circular for a Schwarzschild black hole and remains nearly circular for Kerr black holes. On the other hand, if the quadrupole moment is left as an independent parameter, the ring shape changes significantly and becomes asymmetric. The degree of asymmetry is a direct measure of the violation of the no-hair theorem. I show that the diameter of the ring depends only very weakly on the spin and quadrupole moment of the black hole and can be used to directly measure the mass of the object. In addition, the ring is displaced off center in the image plane in the case of rotating black holes (Beckwith \& Done 2005; see, also, Takahashi 2004), and I show that the displacement is a direct measure of the object's spin, modulo the disk inclination.

In Chapter 7, I show that combining a measurement of the ring diameter with the observations of stars around Sgr $\mathrm{A}^{*}$ can reduce the correlation of current measurements of the mass and distance of Sgr A* (Ghez et al. 2008; Gillessen et al. 2009), and I demonstrate the ability of this approach to refine the mass and distance measurements of Sgr $\mathrm{A}^{*}$. I estimate the precision with which a VLBI array can infer the diameter of the ring of Sgr $\mathrm{A}^{*}$ and use a Bayesian technique to simulate measurements of the mass and diameter of Sgr $\mathrm{A}^{*}$ in conjunction with parameters inferred from the existing data of the orbits of stars at comparable wavelengths. In addition, I argue that the accretion flows of other nearby supermassive black holes are optically thin, allowing for VLBI observations of their 
respective photon rings. I assess the prospects of using this technique to infer the masses of these sources.

As a second application, I show how quasi-periodic variability observed across the electromagnetic spectrum in the emission from black holes can be used to test the no-hair theorem. Quasi-periodic oscillations (QPOs) have been observed in several galactic binaries with the Rossi X-ray Timing Explorer (RXTE; see Remillard \& McClintock 2006; see van der Klis 2006 for definitions and analysis techniques) and in Active Galactic Nuclei (AGN) with XMM-Newton (Gierliński et al. 2008). While potential QPO signals in AGN are often obscured by red noise (Benlloch et al. 2001; Vaughan \& Uttley 2005, 2006), galactic black holes usually reveal much cleaner signals. In the case of galactic black holes, QPOs are transient phenomena that occur during mostly nonthermal states of the black-hole accretion disk and during state transitions; they fall into two general classes: highfrequency (about 40-450 Hz) QPOs and low-frequency (about 0.1-30 Hz) QPOs (Remillard \& McClintock 2006).

The physical origin of the observed QPOs is not well understood. In a thoroughly developed hydrodynamic model, they attributed to normal modes of oscillation trapped by general-relativistic effects in the accretion disks around black holes (see Wagoner 1999 and Kato 2001 for reviews and references therein). Expressions of these modes and the corresponding oscillation frequencies have been derived for the case of modified Newtonian potentials (Kato \& Fukue 1980; Okazaki et al. 1987; Kato 1990; Nowak \& Wagoner 1991, 1992, 1993) and in full general relativity (Perez et al. 1997; Silbergleit et al. 2001; Ortega-Rodríguez et al. 2002). Since these frequencies depend primarily on the mass and the spin of the black hole and only very little on the speed of sound, quasi-periodic oscilla- 
tions provide a laboratory for tests of general relativity (see Psaltis 2003, 2008 for reviews).

Of special interest are gravity modes in the equatorial plane, the so-called $g$ modes (Perez et al. 1997; see, however, Li et al. 2003), which are trapped near the inner edge of the accretion disk, as well as corrugation modes or $c$-modes (e.g., Silbergleit et al. 2001), that precess slowly around the angular momentum vector of the black hole. Both of these modes lead to a modulation of black-hole X-ray spectra; $g$-modes usually cover the largest area of the disk near the temperature maximum, while $c$-modes affect the projected area of the disk (see, e.g., Wagoner 1999). Both the $g$-and the $c$-modes are related directly to the epicyclic frequencies of particles on (nearly) circular equatorial orbits (Perez et al. 1997; Silbergleit et al. 2001). The most robust and observable mode is expected to be the axisymmetric $g$-mode, which has also been seen in various hydrodynamic simulations (Mao et al. 2009; Reynolds \& Miller 2009; Chan 2009; see, also, Wagoner 2008).

In a different approach, since QPOs have been observed in pairs with frequency ratios of $\approx 3 / 2$ in several sources (see Remillard \& McClintock 2006), they have also been modeled as nonlinear resonances among the Keplerian and epicyclic frequencies (Kluźniak \& Abramowicz 2001; Abramowicz et al. 2003). The frequencies predicted by both of these models are consistent with observations and have been used in each model to constrain the spin of the black-hole X-ray binary GRO 1655-40 (Abramowicz \& Kluźniak 2001; Wagoner et al. 2001). Variability has also been observed in the emission of Sgr A* in the radio, millimeter, NIR, and X-ray bands with timescales ranging from minutes to hours (e.g., Baganoff et al. 2001; Aschenbach et al. 2004; Genzel et al. 2003; Ghez et al. 2004; Bélanger et al. 2006; Meyer et al. 2006; Yusef-Zadeh et al. 2006; Marrone et al. 
2006; Hornstein et al. 2007).

In Chapter 8, I derive expressions for the Keplerian and epicyclic frequencies of circular equatorial motion in the quasi-Kerr and in my newly constructed spacetimes. I discuss the properties of these frequencies and analyze their distinct dependencies on the mass and spin of the black hole as well as on the parameter that measures the deviation from the Kerr metric. In particular, I demonstrate how this formalism can be applied in order to test the no-hair theorem with the quasi-periodic variability observed from galactic black holes, AGN, and Sgr A*.

As a third application, I analyze relativistically broadened fluorescent iron lines that originate from the irradiation off the accretion disks of black holes as another mechanism to probe the spacetimes of compact objects. Such iron lines have been observed in both galactic black holes and AGN (see Reynolds \& Nowak 2003; Miller 2007 for reviews). Within general relativity, these line profiles can be used to measure the spins of black holes (Fabian et al. 1989; Stella 1990; Laor 1991; Reynolds \& Nowak 2003; Dovčiak et al. 2004; Beckwith \& Done 2004, 2005; Brenneman \& Reynolds 2006; Reynolds \& Fabian 2008; Dexter \& Agol 2009; Karas \& Sochora 2010; Dauser et al. 2010).

The shape of a particular iron line profile depends on the spin of the black hole, the radial extend of its (geometrically thin) accretion disk, the disk emissivity, and the disk inclination with respect to the line of sight of a distant observer (e.g., Fabian et al. 1989). The key property that allows for measurements of black hole spins is the low-energy tail of a given iron line profile, which depends on the inner disk radius of the accretion disk. This radius, in turn, is assumed to coincide with the ISCO of the spacetime, which depends exclusively on the spin of the black hole in units of its mass. Therefore, the spin of the black hole can be 
measured directly from the position of the ISCO, even if other important parameters of the black hole, such as its mass or distance, are unknown (e.g., Reynolds \& Nowak 2003; Brenneman \& Reynolds 2006; Reynolds \& Fabian 2008).

In Chapter 9, I analyze the prospects of using observations of fluorescent iron line profiles to test the no-hair theorem. I incorporate my new black hole metric into the algorithm developed in Chapter 5, and I simulate iron line profiles over the entire range of spins. I demonstrate that deviations from the Kerr metric lead to shifts of the measured flux primarily at high energies as well as in the low-energy tail of the line profiles. I show that these changes can be significant for a black hole with a fixed value of the spin and estimate the required precision of future X-ray missions in order to be able to test the no-hair theorem with fluorescent iron lines for disks of different inclinations.

I also show, however, that line profiles from black holes with different spins and values of the deviation parameter, chosen in such a way that the spacetimes have the ISCO at the same coordinate radius, are practically indistinguishable even for black holes with high spins. I isolate this effect from the other relativistic properties of the spacetime and show that these properties only contribute to the line shape at the few percent level. I argue that this correlation may be reduced in combination with other observables such as the frequencies of QPOs, which depend only indirectly on the location of the ISCO.

The applications reported in Chapters 6 and 8 are based on the quasi-Kerr metric (Glampedakis \& Babak 2006), which I used initially as my framework for tests of the no-hair theorem. I constructed the Kerr-like metric in Chapter 4 afterwards, which lead to the analysis of the pathologies of Kerr-like metrics in Chapter 3 and which I have used as the more general framework for tests of 
the no-hair theorem ever since. Both frameworks were implemented in the raytracing algorithm in Chapter 5. However, for the analysis in Chapter 9 I used only the version with my newly constructed metric because of its ability to describe rapidly-spinning black holes. The analysis of black-hole images in Chapter 6 still needs to be studied in the context of the new framework. The changes of the existing analysis will be marginal, but my new metric would allow me to extent it to include black holes with arbitrary values of the spin. 


\subsection{Constraining the Size of Large Extra Dimensions}

In the second part of this thesis, I obtain constraints on the size of extra dimensions from the orbital evolution of black-hole X-ray binaries in Randall-Sundrum type braneworld gravity. In the search for the unified theory of all forces, an essential ingredient is the solution of the so-called hierarchy problem. The fundamental scale of gravity, the Planck mass, exceeds the electroweak scale by 16 orders of magnitude. In order to resolve this discrepancy, Arkani-Hamed, Dimopoulos, \& Dvali (1998) suggested that gravity is allowed to propagate in more then three spatial dimensions and is hence "diluted" in our universe. This leads to modifications of gravity at distances that are smaller than those probed by experiments. Indeed, Newton's inverse square law has been tested down to the sub-mm range (Kapner et al. 2007; Geraci et al. 2008), hence verifying that our space is three-dimensional at macroscopic scales. Any modification of gravity involving extra dimensions, therefore, has to ensure that additional space dimensions only affect our world at distances that are smaller than those experimental limits.

Braneworld gravity offers a solution to this problem in the form of two different scenarios. One approach (Arkani-Hamed et al. 1998) is to compactify $n$ extra dimensions at scales smaller than those set by experiment. The fundamental Planck mass can be pushed down to the electroweak scale of about $1 \mathrm{TeV}$, provided the extra dimensions are large enough. For $n$ extra dimensions, the limit is $R \lesssim 10^{30 / n-17} \mathrm{~cm}$. For $n \geq 2$, extra dimensions would have a sub-mm size, which is just at the limit up to which the inverse square law has been verified. This model can also be embedded in string theory (Antoniadis et al. 1998). However, it cannot be tested in astrophysics, because those length scales are well 
below astronomical distances.

A second scenario (Randall \& Sundrum 1999, hereafter RS2) is based on a different idea. The four-dimensional brane with all standard model particles is embedded in an infinite five-dimensional anti-de Sitter space. Deviations from the inverse square law, however, only manifest at distances smaller than the asymptotic curvature radius $L$ of the bulk because the latter is filled with a negative cosmological constant. This setup has dramatic implications for astrophysical black holes.

No stable solutions for black holes on the brane have been found to date. Perturbative solution of the classical bulk equations governing the evolution of black holes in the RS2 scenario that are localized on the brane indicated that black holes are unstable and hence lose energy in the extra dimension (Tanaka 2003). Based on the AdS/CFT correspondence, Tanaka (2003) suggested that stable black holes may not exist on the brane at all. Applying the AdS/CFT correspondence to AdS braneworld models, Emparan, Fabbri, \& Kaloper (2002) conjectured that black holes localized on the brane that are solutions of the classical bulk equations in $A d S_{\mathrm{D}+1}$ with the brane boundary conditions correspond to quantum-corrected black holes in $D$ dimensions. Black holes can then evaporate through the emission of a large number of CFT modes with a lifetime given by (Emparan, GarcíaBellido, \& Kaloper 2003; see, however, Fitzpatrick, Randall, \& Wiseman 2006)

$$
\tau \sim 1.2 \times 10^{2}\left(\frac{M}{M_{\odot}}\right)^{3}\left(\frac{1 \mathrm{~mm}}{L}\right)^{2} \mathrm{yr},
$$

which is only of the order of a hundred thousand years for black holes with a mass $M$ of a few solar masses and an asymptotic curvature $L$ in the sub-mm range. Therefore, astrophysical black holes can radiate away most of their mass 
at cosmologically relevant timescales. This property has been used to constrain $L$ from a kinematic limit on the age of the black hole XTE J1118+480 (Psaltis 2007), yielding $L<80 \mu \mathrm{m}$.

In the RS2 model, the gravitational potential at distances close to $L$ takes the form (RS2)

$$
V(r) \approx-G \frac{m_{1} m_{2}}{r}\left(1+\frac{L^{2}}{r^{2}}\right) .
$$

Adelberger et al. (2007) report a $1 \sigma$-upper limit on $L$ of $11 \mu \mathrm{m}$. A $3 \sigma$-constraint was not reported, but it should be significantly larger and comparable to the $95 \%$ confidence upper bound of $44 \mu \mathrm{m}$ for the size of one compact extra dimension (Kapner et al. 2007). Hereafter, we will take the latter as the current experimental constraint on $L$.

In Chapter 10, I constrain the asymptotic curvature radius $L$ in the RS2 scenario by considering the evaporation of black holes in X-ray binaries. A mass loss of the black hole in the extra dimension leads to an evolution of the orbit, which is potentially measurable. Competing effects are the orbital period evolution caused by magnetic braking and the evolution of the companion star (see, e.g., Verbunt 1993).

In Chapter 11, I use measurements of the orbital periods of the X-ray binaries A0620-00 and XTE J1118+480 from the literature to obtain upper limits on the asymptotic curvature radius $L<161 \mu \mathrm{m}$ and $L<0.97 \mathrm{~mm}$, respectively. I predicted (at the time of publication) that only one additional measurement of the orbital period of XTE J1118+480 would lead to the first detection of orbital evolution in a black-hole binary and impose the tightest constraint on the size of one extra dimension of the order of $35 \mu \mathrm{m}$. In addition, I discuss the potential of A0620-00 as well as of other black hole binaries to constrain the asymptotic cur- 
vature radius $L$ down to a few microns. I summarize the results and conclusions of both parts in Chapter 12. 


\section{CHAPTER 2}

\section{A FrAmeWORK FOR TESTING THE NO-HAIR THEOREM}

In this chapter, I investigate a framework for testing the no-hair theorem with observations in the electromagnetic spectrum. I formulate these tests in terms of a quasi-Kerr metric (Glampedakis \& Babak 2006) that incorporates an independent quadrupole moment but reduces smoothly to the familiar Kerr metric when the deviation at the quadrupole order is set to zero. The central object constitutes a quasi-Kerr black hole (similar to the term bumpy black hole coined by Collins \& Hughes 2004): it deviates from the Kerr metric in (at least) one multipole moment and allows for a test that distinguishes a Kerr black hole from a different type of object.

I compute observables that can be measured directly with either current or near-future instruments and allow for the extraction of at least three independent multipole moments. This approach allows for a two-fold test: If the central object is indeed a black hole, a deviation from the Kerr metric must be zero. If, however, the deviation is measured to be nonzero, then it either has to be a different type of object or general relativity itself breaks down in the strong-field regime very close to the black hole (c.f., Collins \& Hughes 2004; Hughes 2006).

In particular, my framework will enable me to perform a test of the no-hair theorem with the measurement of black-hole images (Chapter 6), quasi-periodic variability (Chapter 8 ), and relativistically broadened iron lines emitted from accretion disks (Chapter 9). All of these observables depend explicitly on the multipole moments of the spacetime. Under the assumption that the compact objects are Kerr black holes, these observables have been used to measure the spins of 
the black holes (e.g., Zhang, Cui, \& Chen 1997; Brenneman \& Reynolds 2006; Broderick et al. 2009a). In the more general case, their properties and respective shapes likewise can be used to constrain the quadrupole moments. Similar approaches have been suggested based on timing observations of pulsar black hole binaries (Wex \& Kopeikin 1999) and on observations of stellar orbits in the vicinity of Sgr A* (Will 2008; Merritt et al. 2009). Most of the contents of this chapter were published in Johannsen \& Psaltis (2010a). 


\subsection{Multipole Moments and the No-Hair Theorem}

In this section, I first discuss multipole expansions of curved spacetimes in general before I focus on the special nature of the Kerr metric. Then I briefly review four approaches to extracting observationally the spacetime parameters of a black hole.

\subsubsection{Multipole Expansions of Curved Spacetimes}

Symmetric systems in flat space are often best described by a set of multipole moments. In theories of gravity like general relativity, however, space is curved due to the presence of matter, and the resulting field equations are highly nonlinear. It is, therefore, not immediately obvious that such a spacetime can actually be characterized by a set of multipole moments.

In Newtonian gravity, the potential $\Phi$ satisfies the Laplace equation

$$
\nabla^{2} \Phi= \begin{cases}4 \pi G \rho & \text { (interior) } \\ 0 & \text { (exterior) }\end{cases}
$$

where $G$ is Newton's constant, and $\rho$ is the mass density. Therefore, the potential $\Phi$ can always be expanded in spherical harmonics $Y_{l m}$ as

$$
\Phi=-G \sum_{l=0}^{\infty} \frac{4 \pi}{2 l+1} \sum_{m=-l}^{l} \frac{M_{l m} Y_{l m}}{r^{l+1}}
$$

with mass multipole moments

$$
M_{l m}=\int_{0}^{r} r^{\prime l+2} d r^{\prime} \oint d \Omega^{\prime} Y_{l m}^{*}\left(\Omega^{\prime}\right) \rho\left(r^{\prime}, \Omega^{\prime}\right) .
$$

In these expressions, I have left the gravitational constant $G$ explicit. I will set this constant as well as the speed of light $c$ to unity for the remainder of this thesis. 
In the curved space of general relativity, however, the vacuum Einstein equations

$$
R_{\mu \nu}-\frac{1}{2} g_{\mu \nu} R=0
$$

have to be solved for the spacetime metric $g_{\mu \nu}$ with the corresponding Ricci tensor $R_{\mu \nu}$ and Ricci scalar $R$. The Einstein equations are nonlinear and, therefore, cannot always be solved in terms of an expansion over orthonormal polynomials. Nonetheless, it can be shown that a multipole expansion of curved spacetime does indeed exist (see Thorne 1980 for a detailed review).

For an asymptotically flat vacuum solution of the Einstein equations, (tensor) multipole moments can be defined based on a conformal compactification of 3-space, if the spacetime is also static (Geroch 1970) or, more generally, if it is stationary (Hansen 1974). In both cases, such a set of multipole moments characterizes the spacetime uniquely (Beig \& Simon 1980, 1981; see also Hauser \& Ernst 1981 and references therein) and obeys an appropriate convergence condition (Bäckdahl \& Herberthson 2006). If the spacetime is also axisymmetric, the multipole moments are given by a bi-infinite series of scalars $M_{l}$ and $S_{l}$, which are interpreted as mass and current multipole moments, respectively. The mass multipole moments are analogous to the multipole moments in Newtonian gravity given by expression (2.3) and are nonzero only for even $l$. The current multipole moments are nonzero only for odd $l$ and arise from the fact that, in general relativity, all forms of energy gravitate (Hansen 1974). Stationary axisymmetric vacuum solutions of the Einstein equations can likewise be generated from a given set of multipole moments (Sibgatullin 1991; Manko \& Sibgatullin 1993).

In general relativity, black-hole spacetimes are asymptotically flat vacuum solutions of the Einstein equations. These spacetimes must also be axisymmetric 
(Hawking 1972) and can therefore be described by a sequence of multipole moments $\left\{M_{l}, S_{l}\right\}$. As a consequence of the no-hair theorem, the Kerr spacetime is the unique black-hole solution within general relativity that contains an event horizon, and all multipole moments of order $l \geq 2$ are determined only by the first two, i.e., by the mass $M=M_{0}$ and the spin $J=S_{1}$. This fact can be expressed mathematically with the relation (Geroch 1970; Hansen 1974)

$$
M_{l}+\mathrm{i} S_{l}=M(\mathrm{i} a)^{l}
$$

where $a=J / M$ is the dimensionless spin parameter.

In the astrophysical context, the fact that the no-hair theorem requires the multipole moments to be locked by expression (2.5) allows for it to be tested quantitatively using observations of black holes. Since the first two multipole moments (i.e., the mass and spin) already specify the entire spacetime, any observable can ultimately depend only on those two moments. Therefore, a promising strategy for testing the no-hair theorem is to measure (at least) three multipole moments of the spacetime of a black hole (Ryan 1995).

2.1.2 Four Approaches to Measuring the Multipole Moments of Curved Spacetimes

To date, four approaches to testing the no-hair theorem have been suggested that are based on either a multipole expansion or a perturbation of the Kerr spacetime. In the following, I briefly review these methods in order to identify the one that is most suitable for my purposes.

The idea to "map" the spacetime of an astrophysical black hole and, therefore, to directly measure its multipole moments was first developed by Ryan (1995). He used an expansion in Geroch-Hansen multipoles in order to design a detection 
mechanism that could, in principle, probe the spacetime of a black hole using extreme mass-ratio inspiral (EMRI) observations. He showed that the values of the multipoles are encoded in several observables that can be measured by future experiments. In particular, these values can be extracted from the evolution of the orbital phase of inspiraling objects (by, e.g., X-ray timing missions) or from the gravitational wave spectrum using LISA (Ryan 1995, 1997a, 1997b).

Ryan (1995) considered a spacetime that is stationary, axisymmetric, and asymptotically flat. The inspiraling object moves gradually along a family of geodesic orbits in the equatorial plane and radiates away its energy and angular momentum adiabatically. The metric takes the form (Papapetrou 1953)

$$
d s^{2}=-F(d t-\omega d \phi)^{2}+\frac{1}{F}\left[e^{2 \gamma}\left(d \rho^{2}+d z^{2}\right)+\rho^{2} d \phi^{2}\right],
$$

using cylindrical Weyl coordinates $(t, \rho, \phi, z)$. The functions $F, \gamma$, and $\omega$ depend on the coordinates $\rho$ and $z$ and can be generated from the Ernst potential (Ernst 1968)

$$
\mathcal{E}=F+i \Psi=\frac{\sqrt{\rho^{2}+z^{2}}-\xi}{\sqrt{\rho^{2}+z^{2}}+\xi} .
$$

The function $\xi$ can be expanded as (Fodor, Hoenselaers \& Perjés 1989)

$$
\xi=\sum_{j, k=0}^{\infty} a_{j k} \frac{\rho^{j} z^{k}}{\left(\rho^{2}+z^{2}\right)^{j+k}}
$$

which specifies $F(\rho, z)$. The coefficients $a_{j k}$ are directly related to the multipole moments of the spacetime given by the metric in expression (2.6). The remaining functions $\omega(\rho, z)$ and $\gamma(\rho, z)$ are then obtained in the form of two integrals (Dietz 1984; Wald 1984, pp. 165-67). Barack \& Cutler (2004; 2007) used approximate waveforms to extend Ryan's analysis for generic orbits and included modulations caused by LISA satellites. Li \& Lovelace (2008) generalized Ryan's approach to 
include tidal coupling between the inspiraling and the central object. One might interject at this point (see also Collins \& Hughes 2004) that a $1 / r$-expansion such as the one exhibited by equation (2.8) spoils the promise of an approach that is based on the multipole moments of the spacetime. The potential difficulty is that a large number of such moments will be required to describe the spacetime as one approaches the event horizon.

In an alternative approach, Collins \& Hughes (2004) constructed spacetimes of perturbed Schwarzschild black holes (bumpy black holes). These spacetimes have the usual symmetries of being stationary, axisymmetric, and asymptotically flat, and the black hole is effectively perturbed by the presence of additional fields exterior to the black-hole horizon. For these configurations Collins \& Hughes (2004) calculated the periapse precession of equatorial orbits and found a significant (factor $\sim 10$ ) increase of the precession in the strong-field regime.

Their starting point was the Weyl metric (Weyl 1918)

$$
d s^{2}=-e^{2 \psi} d t^{2}+e^{2 \gamma-2 \psi}\left(d \rho^{2}+d z^{2}\right)+e^{-2 \psi} \rho^{2} d \phi^{2},
$$

which is identical to the metric (2.6) with the substitutions $F=e^{2 \psi}$ and $\omega=0$. The quantities $\psi$ and $\gamma$ are solutions to the vacuum Einstein equations (Collins \& Hughes 2004; see also Suen, Price \& Redmount 1988).

Then Collins \& Hughes (2004) introduced small perturbations as $\psi=\psi_{0}+\psi_{1}$ and $\gamma=\gamma_{0}+\gamma_{1}$, where $\psi_{0}$ and $\gamma_{0}$ are the corresponding expressions of the Schwarzschild metric, and solved the Einstein equations keeping only leading order terms in the perturbation. (Note that this technique does not make explicit use of the multipole moments.) This approach was recently generalized by Vigeland \& Hughes (2010) who considered similar perturbations in the metric of 
rotating black holes.

A third approach is based on the Manko-Novikov metric (Manko \& Novikov 1992). This metric is stationary, axisymmetric, and asymptotically flat and is also a solution of the vacuum Einstein equations. It is a parametric extension of the Kerr metric with a different set of multipole moments. In this metric, mass and current multipole moments are coupled. In addition to the usual first two moments, i.e., mass and spin, the mass multipole moments of order $l \geq 2$ are free parameters. They in turn specify the current multipole moments. In general, this metric has no event horizon but contains a naked singularity at the center (Manko \& Novikov 1992). Gair et al. (2008) studied a subclass of the MankoNovikov metric analyzing possible observational signatures of ergodic non-Kerr EMRIs. Apostolatos, Lukes-Gerakopoulos, \& Contopoulos (2009) showed that the appearance of Birkhoff chains in the neighborhood of resonant tori (due to the Poincaré-Birkhoff theorem, Poincaré 1912; Birkhoff 1913) would lead to such a modification of a gravitational-wave measurement.

Finally, in a fourth approach, Glampedakis \& Babak (2006) considered perturbations of the Kerr metric that still satisfy the vacuum Einstein equations by exploiting the properties of the Hartle-Thorne metric (Hartle 1967; Hartle \& Thorne 1968). This metric, in Hartle-Thorne coordinates, describes the spacetime outside any slowly rotating compact object within general relativity and was originally designed for neutron stars. It provides an expansion of a general spacetime up to the quadrupole order and has a quadrupole moment $Q$ that is independent of both mass and spin (Hartle \& Thorne 1968). Glampedakis \& Babak (2006) constructed a quasi-Kerr extension to the Kerr metric by choosing the (dimensionless) quadrupole moment to be $q_{\mathrm{Kerr}}-\epsilon$, where $\epsilon$ parametrizes a potential devia- 
tion from the Kerr metric and $q_{\mathrm{Kerr}}=-J^{2} / M^{4}=-a^{2} / M^{2}$. In this parametrization, the quadrupole moment is given by (Glampedakis \& Babak 2006)

$$
Q=-M\left(a^{2}+\epsilon M^{2}\right) .
$$

I verified explicitly that this metric is a solution of the vacuum Einstein equations up to the quadrupole order by showing that the Ricci tensor vanishes.

The quasi-Kerr metric is stationary, axisymmetric, and asymptotically flat and reduces to the Kerr metric in the limit $\epsilon \rightarrow 0$. The particular choice of BoyerLindquist coordinates by Glampedakis \& Babak (2006) is convenient, because it preserves the special Petrov-type D character of the Kerr part. Similar approaches have also been analyzed in the context of neutron stars (Laarakkers \& Poisson 1999; Berti \& Stergioulas 2004), rigidly rotating stars (Bradley \& Fodor 2009), and naked singularities (Bini et al. 2009).

For equatorial orbits in the quasi-Kerr metric, Glampedakis \& Babak (2006) calculated the periastron precession and constructed 'kludge' gravitational waveforms as a function of the parameter $\epsilon$. These waveforms can be significantly different from the expected Kerr signal even for small changes of the quadrupole moment. They identified, however, a confusion problem, because it is possible to match quasi-Kerr waveforms of one set of orbital parameters with the Kerr template of a different set of orbital parameters.

The fact that the quasi-Kerr metric is a valid solution of the Einstein equations even for non-Kerr values of the quadrupole moment allows us to design a self-consistent test of the no-hair theorem and of the nature of the compact object within general relativity. A disadvantage of the quasi-Kerr metric is the restriction that it cannot be used for a description of rapidly spinning black holes. The 
most promising approach will be, of course, to perform the tests of the no-hair theorem with all of the above parametrizations of spacetime in order to explore the robustness of the results. Nonetheless, the fact that the quasi-Kerr metric contains an independent quadrupole multipole moment makes it a natural and well-suited environment for a test of the no-hair theorem, which I adopt below. 


\subsection{Defining a Framework For Testing the No-Hair Theorem}

In this section, I formulate my framework for testing the no-hair theorem with observations of black holes in the electromagnetic spectrum. I use the quasiKerr metric (Glampedakis \& Babak 2006) as the underlying spacetime and develop a method from which I derive observables parametrized by an independent quadrupole moment that is potentially different from the value predicted by the no-hair theorem.

I name the central object described by such a metric a quasi-Kerr black hole (similar to the term bumpy black hole, c.f. Collins \& Hughes 2004). This is a black hole within general relativity only if the deviation of all its multipole moments from the respective values in the Kerr spacetime are zero. Similarly to the case of EMRI observations (Collins \& Hughes 2004; Hughes 2006), my approach may constitute a null-hypothesis test: If the central object is indeed a generalrelativistic black hole, then it must have the multipole structure of the Kerr metric given by relation (2.5). If a deviation of the multipole moments from this expression is detected, the object cannot be a general-relativistic black hole. In that case, the object is either a star or, if it is otherwise known to posses an event horizon, a black hole with a different set of multipole moments, which, therefore, violates the no-hair theorem. Contrary to gravitational-wave analyses, however, which rely explicitly on the Einstein equations, my framework requires only the metric and the Einstein Equivalence Principle. Thus, it can also be viewed as a self-consistent test of general relativity in the strong-field regime.

In the following, I specify the exact form of the quasi-Kerr spacetime and I estimate its range of applicability. This metric has three independent multipole moments (mass, spin, and quadrupole moment). The deviation of the quadrupole 
moment from the Kerr value is written in terms of the dimensionless parameter $\epsilon$, so that the full quadrupole moment is given by relation (2.10).

In Boyer-Lindquist coordinates, the Kerr metric $g_{\mathrm{ab}}^{\mathrm{K}}$ takes the form (e.g., Bardeen, Press, \& Teukolsky 1972)

$$
\begin{gathered}
d s^{2}=-\left(1-\frac{2 M r}{\Sigma}\right) d t^{2}-\left(\frac{4 M a r \sin ^{2} \theta}{\Sigma}\right) d t d \phi \\
+\left(\frac{\Sigma}{\Delta}\right) d r^{2}+\Sigma d \theta^{2}+\left(r^{2}+a^{2}+\frac{2 M a^{2} r \sin ^{2} \theta}{\Sigma}\right) \sin ^{2} \theta d \phi^{2}
\end{gathered}
$$

with

$$
\begin{gathered}
\Delta \equiv r^{2}-2 M r+a^{2}, \\
\Sigma \equiv r^{2}+a^{2} \cos ^{2} \theta .
\end{gathered}
$$

The quadrupolar correction is determined by choosing a quadrupole moment of the form (2.10) in the Hartle-Thorne metric (Hartle \& Thorne 1968). Then, the quasi-Kerr metric $g_{\mathrm{ab}}^{\mathrm{QK}}$ in Boyer-Lindquist coordinates is given by (Glampedakis \& Babak 2006)

$$
g_{\mathrm{ab}}^{\mathrm{QK}}=g_{\mathrm{ab}}^{\mathrm{K}}+\epsilon h_{\mathrm{ab}}
$$

In contravariant form, $h^{\text {ab }}$ is

$$
\begin{aligned}
h^{\mathrm{tt}} & =(1-2 M / r)^{-1}\left[\left(1-3 \cos ^{2} \theta\right) \mathcal{F}_{1}(r)\right], \\
h^{\mathrm{rr}} & =(1-2 M / r)\left[\left(1-3 \cos ^{2} \theta\right) \mathcal{F}_{1}(r)\right], \\
h^{\theta \theta} & =-\frac{1}{r^{2}}\left[\left(1-3 \cos ^{2} \theta\right) \mathcal{F}_{2}(r)\right], \\
h^{\phi \phi} & =-\frac{1}{r^{2} \sin ^{2} \theta}\left[\left(1-3 \cos ^{2} \theta\right) \mathcal{F}_{2}(r)\right], \\
h^{\mathrm{t} \phi} & =0
\end{aligned}
$$

The functions $\mathcal{F}_{1,2}(r)$ are given in Appendix A of Glampedakis \& Babak (2006). Note that, although only the quadrupole moment of the spacetime has been al- 
tered, the unperturbed spacetime is formally correct up to the maximum value of the spin.

In the case of the regular Kerr metric (in Boyer-Lindquist coordinates), its Petrov-type D character ensures the existence of four constants of motion: the particle rest mass $\mu$, its energy $E$, the angular momentum about the z-axis $L_{z}$, and the Carter constant (Carter 1968). Equivalently, the Hamilton-Jacobi equation is separable in all four coordinates, i.e., time $t$, radius $r$, and angles $\theta$ and $\phi$, and the equations of motion are reduced to quadratures (see, e.g., Chandrasekhar 1983). This special property greatly simplifies the analysis of a variety of astrophysical applications in the Kerr geometry.

The quasi-Kerr metric in contrast admits full separability of the HamiltonJacobi equation only for equatorial orbits. For generic orbits, the Carter constant is lost. An approximate solution can be found in the neighborhood of the central object using the action-angle formalism of canonical perturbation theory (Glampedakis \& Babak 2006). Unfortunately, this is no longer possible for trajectories that escape to infinity, such as those of photons that reach a distant observer. Consequently, the equations of motion have to be solved numerically. Berti et al. (2005) pointed out quantitatively how a deviation from the Kerr quadrupole moment alters the Petrov type of a spacetime.

Before embarking into the study of the properties of the quasi-Kerr metric that are relevant for observational tests of the no-hair theorem, it is important to check that the multipole expansion of the metric is well-behaved at small radii. An approach based on a multipole expansion breaks down in the limit $r \rightarrow 0$, and a higher number of multipole moments must be kept in order for the expansion to accurately describe the black-hole spacetime near the event horizon (Ryan 1995; 
Collins \& Hughes 2004). The spacetime at horizon scales, however, is most interesting for tests of the no-hair theorem. In the following, I estimate the range of validity of the quasi-Kerr metric. A formal bound on the multipole moments of stationary spacetimes can be found in Bäckdahl \& Herberthson (2006), but this is beyond the scope of this chapter.

The validity of the expansion of the metric considered above breaks down at a critical radius $r_{\mathrm{c}}$ that depends both on the spin $a$ and the parameter $\epsilon$. This cutoff occurs at the radius at which terms of order $\epsilon^{2}$ and $\epsilon a$ can no longer be neglected against the correction terms proportional to $\epsilon$. Unfortunately, these higher order terms cannot be calculated uniquely in the approach of Glampedakis and Babak (2006). In the Kerr metric, the expansion of the diagonal elements in the spin parameter $a$ has nonvanishing corrections only at order $a^{4}$. These terms are always smaller (in magnitude) than the terms at order $a^{2}$ for $r>2 M$ and are negligible at radii $r \gtrsim 2.5 M$. Therefore, in order to estimate the value of the critical radius $r_{\mathrm{c}}$, I compare the quadrupolar correction terms of order $\epsilon$ in the quasi-Kerr metric given by expression (2.13) to the respective elements of the Kerr metric in expression (2.11) up to the quadrupole order.

The metric element which is affected most by the correction that is linear in the parameter $\epsilon$ is $g_{\mathrm{rr}}^{\mathrm{QK}}$. In Figure 1, I plot the radius at which the absolute value of the quadrupolar correction equals (solid lines) the absolute value of the Kerr element $g_{\mathrm{rr}}^{\mathrm{K}}$ up to order $a^{2}$ as a function of the spin $a$. At this radius, the element $g_{\mathrm{rr}}^{\mathrm{QK}}$ changes sign if $\epsilon>0$. This unphysical property renders the metric nonLorentzian and leads to a reflection of infalling particles. ${ }^{1}$ I estimate the cutoff $r_{\mathrm{c}}$ as the radius at which the absolute value of the quadrupolar correction equals

\footnotetext{
${ }^{1}$ I thank A. Broderick for pointing this out.
} 

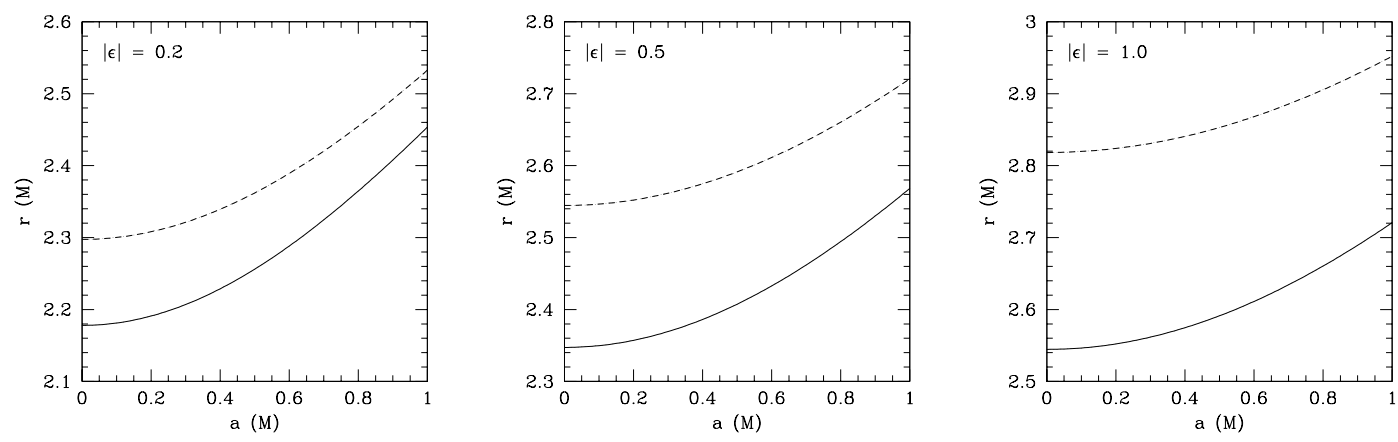

Figure 2.1 The radius $r$ at which the absolute value of the quadrupolar correction of the element $g_{\mathrm{rr}}^{\mathrm{QK}}$ is equal to (solid lines) $100 \%$ and (dashed lines) $50 \%$ of the absolute value of the element $g_{\mathrm{rr}}^{\mathrm{K}}$ expanded to order $a^{2}$. At the radii along the solid lines, the metric element $g_{\mathrm{rr}}^{\mathrm{QK}}$ changes sign if $\epsilon>0$ and the metric becomes non-Lorentzian.

$50 \%$ of the absolute value of the Kerr element $g_{\mathrm{rr}}^{\mathrm{K}}$ up to order $a^{2}$ (Figure 1, dashed lines). Note that these validity constraints are necessary but not sufficient.

The excluded domain $r<r_{\mathrm{c}}$ lies inside the circular photon orbit of the Kerr metric for $a \lesssim 0.4$ and $\epsilon \lesssim 0.5$ (c.f., Bardeen et al. 1972). Therefore, in the quasiKerr metric, I expect the fraction of photons from this region that reach an observer at infinity to be negligible. The only exception are photons from further out in the accretion disk that are strongly lensed by the black hole and approach the event horizon closely.

While higher order terms of the spin $a$ in the quasi-Kerr metric are negligible for values of the radius $r \gtrsim 2.5 \mathrm{M}$, this may not be the case for terms of order $\epsilon a$ if the spin parameter $a$ is too large. I will, therefore, limit my discussion to values of the spin $a \leq 0.4$. For black holes with this amount of spin and a value of the parameter $\epsilon \approx 0.5$, the critical radius $r<r_{\mathrm{c}}$ is comparable to the radius of the circular photon orbit in the Kerr metric. 
An additional complication arises from a singularity at $r_{\mathrm{s}}=2 M$. This singularity is a relic of the expansion in the Hartle-Thorne metric. It vanishes in the Kerr metric, where all divergences cancel, hence illustrating the peculiar nature of that metric. Since the singularity is located at $r_{\mathrm{s}}<r_{\mathrm{c}}$, it plays only a very minor role in the following discussion. It does, however, effect the estimate of the critical radius $r_{\mathrm{c}}$ and therefore would make a different choice of the coordinates desirable.

Technically speaking, my estimate for the critical radius $r_{\mathrm{c}}$ is only valid for the elements of the quasi-Kerr metric $g_{\mathrm{ab}}^{\mathrm{QK}}$, but not for its derivatives. In the case of large quadrupolar corrections, $|\epsilon| \gtrsim a^{2}$, the corresponding Christoffel symbols depend significantly on the value of the parameter $\epsilon$ at radii larger than the critical radius, and the expansion to linear order in the parameter $\epsilon$ is no longer perturbative.

My approach hereafter is to numerically integrate the geodesic equation in the quasi-Kerr spacetime for photons that are emitted from an accretion flow around the black hole and reach a distant observer. The geodesic equation takes the form

$$
\frac{d^{2} x^{\alpha}}{d \lambda^{2}}=-\Gamma_{\beta \gamma}^{\alpha} \frac{d x^{\beta}}{d \lambda} \frac{d x^{\gamma}}{d \lambda}
$$

where $x^{\alpha}=(t, r, \theta, \phi)$ are the coordinates of a given photon, $\frac{d x^{\alpha}}{d \lambda}=u^{\alpha}$ its 4-velocity with $u \cdot u=0$, and $\lambda$ is an affine parameter. Here, the Christoffel symbols $\Gamma_{\beta \gamma}^{\alpha}$ have been expanded to first order in $\epsilon$ neglecting terms of order $\epsilon a$. 


\subsection{Properties of the Quasi-Kerr Metric}

In this section, I analyze some of the properties of the quasi-Kerr metric. Glampedakis \& Babak (2006) obtained analytical expressions for equatorial orbits (where the Hamilton-Jacobi equation separates) as well as approximations for generic orbits in the vicinity of the black hole using canonical perturbation theory. Abramowicz et al. (2003) calculated approximate expressions for circular geodesics in the Hartle-Thorne metric (see, however, Berti et al. 2005 and Glampedakis \& Babak 2006). I use, instead, a numerical integration of the geodesic equation for photons and particles in order to study the properties of orbits that determine observables in the electromagnetic spectrum.

All expressions in this section are calculated to the quadrupole order, i.e., they have been expanded in $\epsilon$ to first order, and terms of order $\epsilon a$ Ire neglected. Note that (following Glampedakis \& Babak 2006) I do not expand in the spin parameter a. This way I leave the Kerr part of all expressions unchanged and can study deviations from the Kerr metric by only changing the quadrupole moment.

\subsubsection{The Event Horizon and Static Limit}

I calculate the locations of the event horizon and of the static limit directly from the metric (2.13). I find the static limit by solving

$$
g_{\mathrm{tt}}=0
$$

for $(r, \theta)$. The corresponding condition for the horizon is

$$
g^{\mathrm{rr}}=0
$$

In Figure 2.2 I plot the radius of the event horizon in the equatorial plane as a function of the spin $a$ for several values of the parameter $\epsilon$. I also plot the ra- 
dius of the static limit in the equatorial plane as a function of the parameter $\epsilon$. The horizon generally lies outside of the perturbative domain of the quasi-Kerr metric. If higher order terms are included, the radius of the event horizon might be slightly shifted or may even not exist at all (c.f., Manko \& Novikov 1992). The radius of the horizon increases with increasing positive values of the parameter $\epsilon$. If $\epsilon$ is negative, equation (2.17) has no solution in general indicating the existence of a naked singularity. The radius of the horizon decreases as the spin $a$ gets larger as expected from the Kerr part of the metric. The static limit in the equatorial plane is independent of the spin $a$ and increases with increasing values of the parameter $\epsilon$. It likewise lies in the nonperturbative domain of the metric, but it gives a first insight into the importance of the quadrupolar correction parameter $\epsilon$ for relativistic boosting (c.f. Section 2.3.4). Note that the horizon is not regular for values of the angle $\theta \sim \pi / 4$ or $\theta \sim 3 \pi / 4$.

\subsubsection{The Innermost Stable Circular and Marginally Bound Orbits}

I now derive analytic expressions for the energy and angular momentum of massive particles on a circular equatorial orbit around a black hole in the quasi-Kerr metric. From these, I calculate the respective radii of the marginally bound orbit and of the innermost stable circular orbit (ISCO). The derivations are similar to the ones in Bardeen (1973) for the Kerr metric. All expressions are expanded to first order in $\epsilon$ omitting terms of order $\epsilon a$.

The quasi-Kerr metric is stationary and axisymmetric, and therefore generally admits three conserved quantities. For a particle with four-momentum

$$
p^{\alpha}=\mu \frac{d x^{\alpha}}{d \tau}
$$

these are its rest mass $\mu$, energy $E=-p_{\mathrm{t}}$, and angular momentum about the 

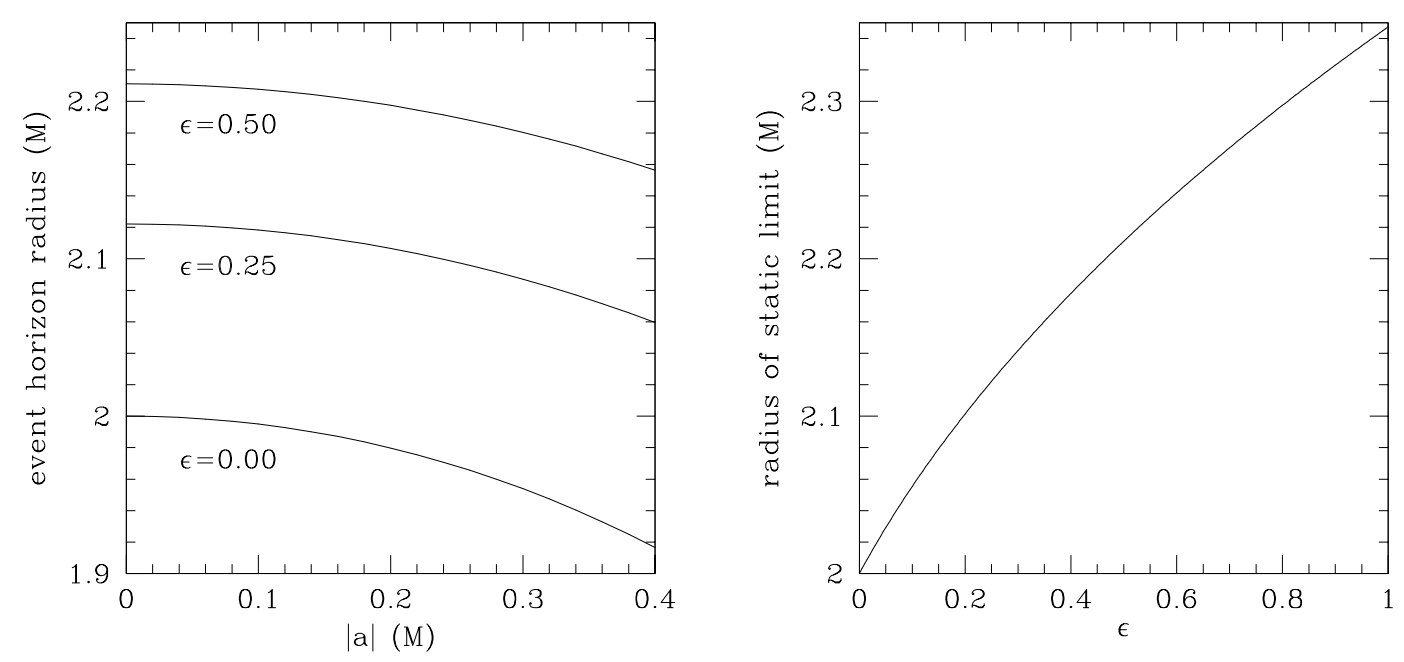

Figure 2.2 (Left:) The location of the event horizon in the equatorial plane of a quasi-Kerr black hole as a function of the absolute value of the spin $a$ for several values of the quadrupolar correction parameter $\epsilon$. Increasing the value of the spin decreases the radius of the event horizon, while increasing the value of the parameter $\epsilon$ increases that radius. (Right:) The equatorial static limit of a quasiKerr black hole as a function of the quadrupolar correction parameter $\epsilon$. A larger value of $\epsilon$ increases the radius of the static limit. In the equatorial plane, the static limit is independent of the spin $a$.

$z$-axis $L_{\mathrm{z}}=p_{\phi}$. Solving the equation

$$
p \cdot p=-\mu^{2}
$$

in the equatorial plane for the radial derivative and substituting the constants of the motion yields

$$
\begin{gathered}
r^{3}\left(\frac{d r}{d \tau}\right)^{2} \equiv R(r) \\
\equiv-4 a M E L_{\mathrm{z}}-(r-2 M) L_{\mathrm{z}}^{2}-\mu^{2} r\left[a^{2}+r(r-2 M)\right]+\left[r^{3}+a^{2}(r+2 M)\right] E^{2} \\
-\frac{5 \epsilon}{16 M^{2} r}\left\{2 M \left[\mu^{2} r^{2}\left(2 M^{3}+4 M^{2} r-9 M r^{2}+3 r^{3}\right)\right.\right. \\
\left.+2\left(3 M^{3}-2 M^{2} r-6 M r^{2}+3 r^{3}\right) L_{\mathrm{z}}^{2}\right]
\end{gathered}
$$




$$
\left.+3 r(r-2 M)\left[-\mu^{2} r^{3}(r-2 M)+2\left(M^{2}+M r-r^{2}\right) L_{\mathrm{z}}^{2}\right] \ln \left(\frac{r}{r-2 M}\right)\right\} .
$$

I obtain the expressions for $E$ and $L_{\mathrm{z}}$ by solving the system of equations

$$
\begin{aligned}
R(r) & =0, \\
\frac{d}{d r} R(r) & =0 .
\end{aligned}
$$

After a nontrivial amount of algebra I find the energy

$$
\begin{gathered}
\frac{E}{\mu}=\frac{r^{3 / 2}-2 M r^{1 / 2} \pm a M^{1 / 2}}{r^{3 / 4} \sqrt{r^{3 / 2}-3 M r^{1 / 2} \pm 2 a M^{1 / 2}}} \\
-\frac{5 \epsilon}{32 M^{2} r^{3 / 2}(r-3 M)^{3 / 2}}\left[2 M\left(6 M^{4}+14 M^{3} r-41 M^{2} r^{2}+27 M r^{3}-6 r^{4}\right)\right. \\
\left.+r^{2}\left(6 r^{3}-33 M r^{2}+66 M^{2} r-48 M^{3}\right) \ln \left(\frac{r}{r-2 M}\right)\right]
\end{gathered}
$$

and angular momentum about the $z$-axis

$$
\begin{gathered}
\frac{L_{\mathrm{z}}}{\mu}= \pm \frac{M^{1 / 2}\left(r^{2} \mp 2 a M^{1 / 2} r^{1 / 2}+a^{2}\right)}{r^{3 / 4} \sqrt{r^{3 / 2}-3 M r^{1 / 2} \pm 2 a M^{1 / 2}}} \\
\mp \frac{5 \epsilon}{32 M^{5 / 2}(r-3 M)^{3 / 2}}\left[2 M\left(6 M^{4}-7 M^{3} r-16 M^{2} r^{2}+12 M r^{3}-3 r^{4}\right)\right. \\
\left.+3 r\left(r^{4}-5 M r^{3}+9 M^{2} r^{2}-2 M^{3} r-6 M^{4}\right) \ln \left(\frac{r}{r-2 M}\right)\right] .
\end{gathered}
$$

For $\epsilon=0$ these expressions are identical to equations (17) and (18) in Bardeen (1973).

From the equation (2.23) for the energy I obtain the radius of the marginally bound orbit by numerically solving

$$
\frac{E}{\mu}=1
$$

and of the innermost stable circular orbit (ISCO) from

$$
\frac{d E}{d r}=0
$$


Similar calculations have also been performed by Shibata \& Sasaki (1998) and Berti \& Stergioulas (2004).

Figure 2.3 shows the radius of the innermost stable circular orbit, $r_{\mathrm{ISCO}}$, and the radius of the marginally bound orbit, $r_{\mathrm{mb}}$, for massive particles as a function of the spin $a$ for several values of the parameter $\epsilon$. Both radii increase with increasing positive $\epsilon$ and decreasing spin $a$. If $\epsilon$ is significantly smaller than zero, the expansions of the energy and the angular momentum in expressions (2.23) and (2.24) are not well defined, because the correction proportional to $\epsilon$ diverges at $r=3 M$. Therefore, I only plot the radius of the ISCO and of the marginally bound orbit for values of the parameter $\epsilon \geq 0$.

The ISCO is of special importance, because it determines the inner edge of the accretion disk of the black hole. If a massive particle from the accretion disk enters the region where $r<r_{\mathrm{ISCO}}$, it will quickly plunge into the black hole. In the Kerr geometry, the ISCO only depends on the mass and the spin of the black hole. A measurement of this radius for a black hole with known mass therefore allows for the spin to be measured (e.g., Brenneman \& Reynolds 2006; Zhang et al. 1997; Shaffee et al. 2006). In the quasi-Kerr metric, it also depends on the quadrupole moment (2.10) and therefore on $\epsilon$. The significant dependence of the ISCO on the quadrupolar parameter $\epsilon$ will have a strong impact on many astrophysical applications such as black-hole images, iron lines, or disk spectra (see below).

\subsubsection{Photon Trajectories}

Close to the black hole, the quadrupole moment becomes important and affects the trajectories of photons and massive particles in the accretion disk. Since the quasi-Kerr metric is of Petrov-type $\mathrm{D}$ in the equatorial plane, these orbits remain equatorial. I integrated the geodesic equation (2.15) numerically using a Runge- 

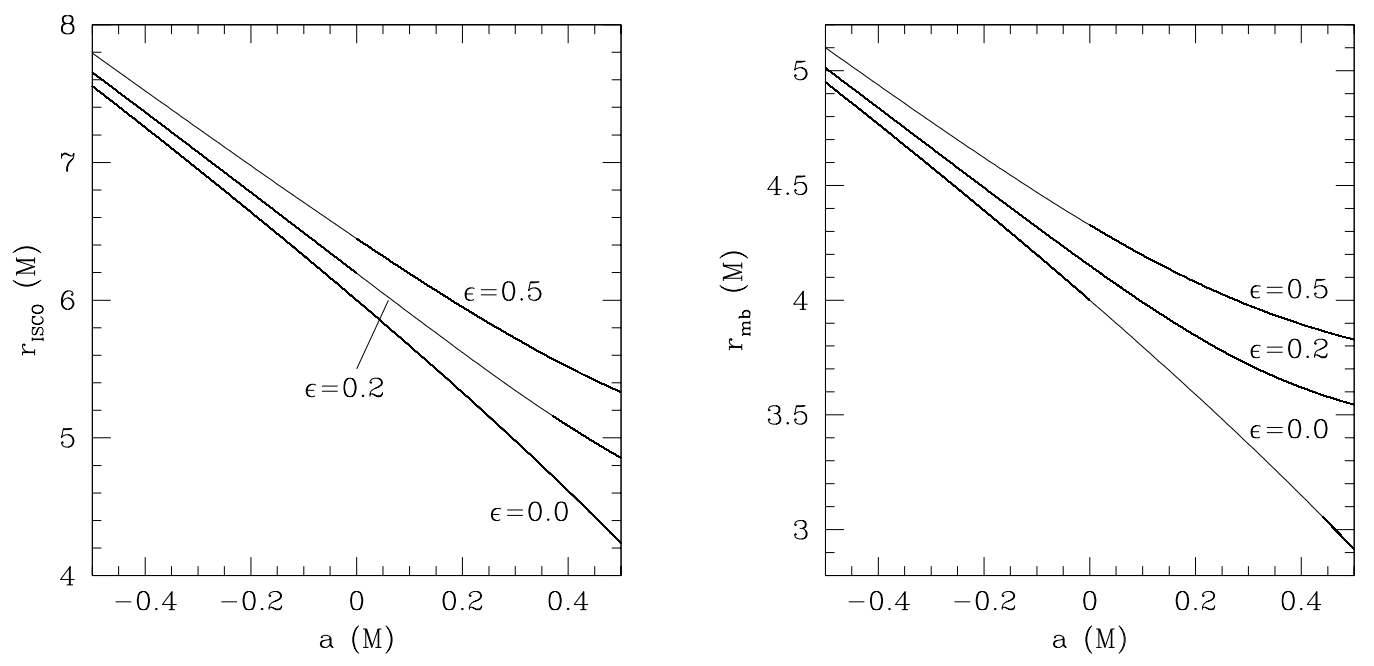

Figure 2.3 The radius of (left) the innermost stable circular orbit and (right) the marginally bound orbit for a massive particle orbiting a quasi-Kerr black hole as a function of the spin $a$ for several values of the quadrupolar correction parameter $\epsilon$. In both cases, a larger spin causes the respective orbits to be closer to the black hole, while an increasing value of the parameter $\epsilon$ moves the respective orbits to larger radii.

Kutta method with adaptive stepsize for several equatorial photon orbits.

Figure 2.4 illustrates the impact of the interplay between the black hole's spin and quadrupole moment on the equatorial orbits. A positive spin $a$ corresponds to counter-clockwise rotation of the black hole. A collection of light rays approaches the black hole parallel to the $x$-axis from the right. The figure is scale invariant with respect to the black-hole mass $M$, and all quantities are expressed in appropriate powers of the mass $M$. The shaded region corresponds to the excluded domain $r<2.6 M$.

For the Kerr black hole, trajectories are shifted due to the usual frame dragging. For the quasi-Kerr black holes, a change of the quadrupole moment away 

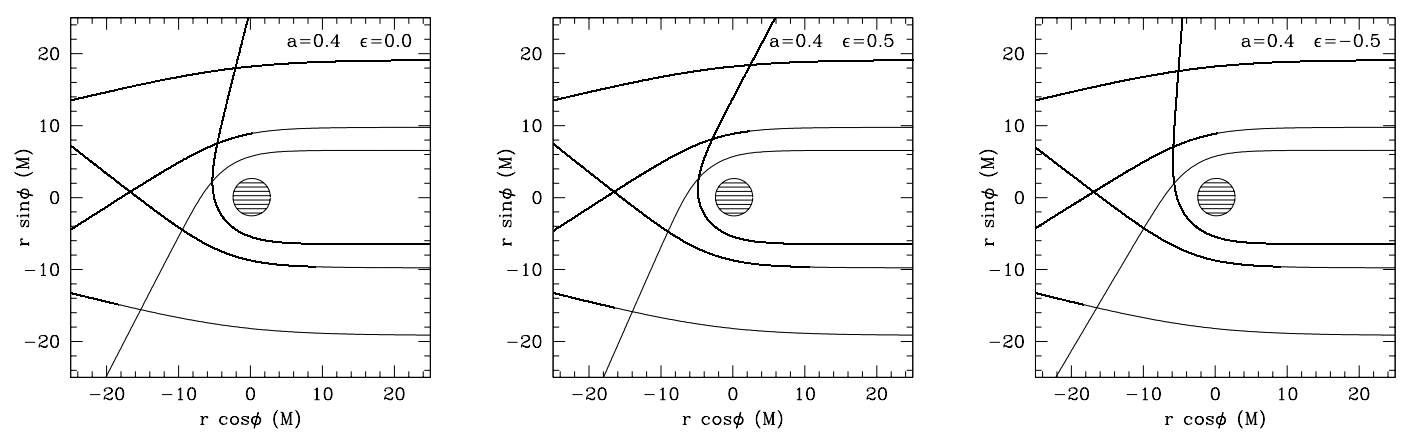

Figure 2.4 Several equatorial photon trajectories around a Kerr black hole (left) and quasi-Kerr black holes with quadrupolar parameter $\epsilon=0.5$ (center) and $\epsilon=-0.5$ (right), respectively. The spin parameter has a value of $a=0.4$ in all cases. Photons approach the black hole from the right. The trajectories experience the strongest bent the closer they approach the black hole. Frame dragging shifts orbits in the direction of (the counter-clockwise) rotation of the black hole, while an enhanced/decreased quadrupole moment increases/decreases the light bending in the immediate vicinity of the black hole. The shaded region marks the excluded domain $r<2.6 \mathrm{M}$.

from its value in the Kerr metric either increases or decreases the amount of light bending. For a positive value of the quadrupolar correction $\epsilon$, trajectories are shifted further in the clockwise direction. For a negative value of the parameter $\epsilon$, the reverse is the case for trajectories that approach the black hole closely. As expected, in both cases, an alteration of the quadrupole moment only effects trajectories in the immediate vicinity of the black hole, while photon trajectories further away from the black hole are dominated by the spin.

In Figure 2.5 I show the effect of varying the quadrupole moment on one trajectory in particular. I held the spin fixed at $a=0.4$ and show photon trajectories for $\epsilon=-0.5,0.0,0.3,0.5$. The effect of the quadrupole moment becomes very apparent once the photons approach a radius of $r \sim 3 M$. A positive value of 


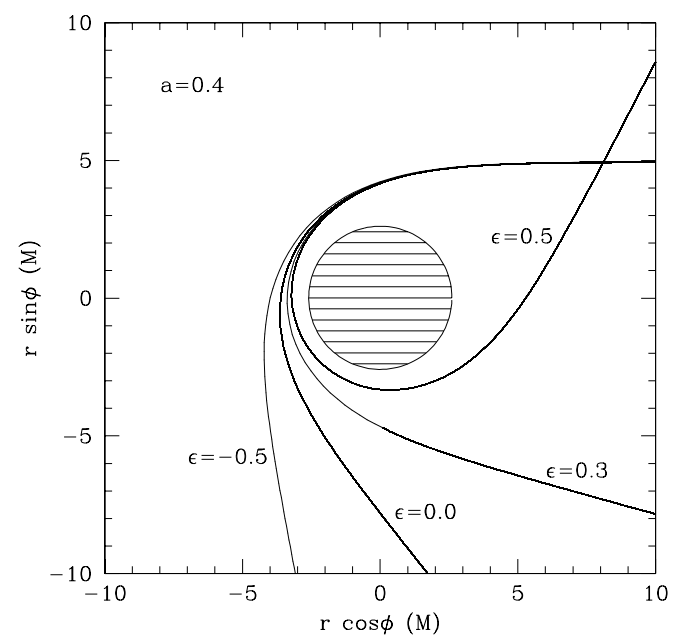

Figure 2.5 Scattering of equatorial photon trajectories by a quasi-Kerr black hole with a (counter-clockwise) spin $a=0.4$ for several values of the quadrupolar correction parameter $\epsilon$. The photons approach the black hole from the right. The shaded region marks the excluded domain $r<2.6 M$. The larger the value of the parameter $\epsilon$, the stronger the amount of light bending.

$\epsilon$ increases the amount of light bending, while that effect deceases for negative values of $\epsilon$ compared to the Kerr trajectory. Note that for this particular orbit, the asymptotic azimuthal angle $\phi_{\infty}$ differs by more than $\pi / 2$ for the orbits with $\epsilon=-0.5$ and $\epsilon=0.5$, respectively. These effects will significantly alter the shadows of black holes as well as the details of accretion flow spectra.

\subsubsection{Gravitational Redshift and Lorentz Boosts}

In this section I investigate the dependence of gravitational redshift and Lorentz boosting of photons emitted in the quasi-Kerr spacetime on the quadrupolar parameter $\epsilon$. These effects are critical for predicting the observational appearance of astrophysical processes in the vicinity of black holes. In particular, they de- 


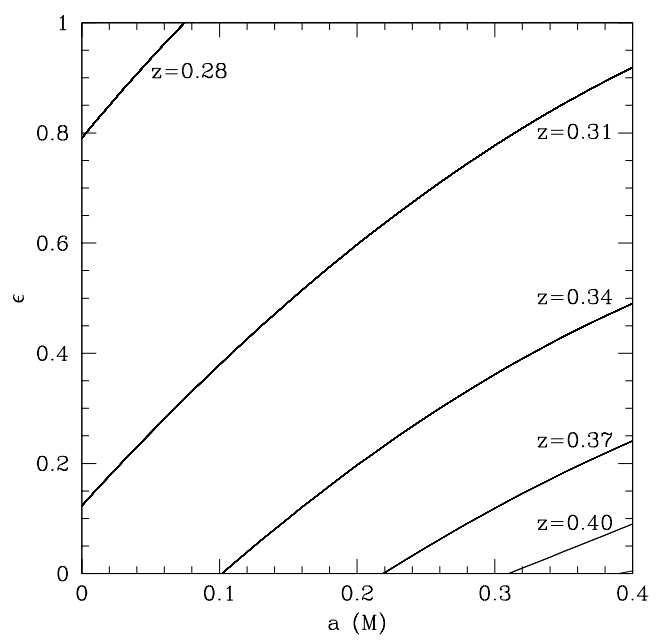

Figure 2.6 Contours of constant redshift $z$ experienced by a photon emitted by a particle on the (prograde) ISCO as viewed by an observer at rest at infinity as a function of black-hole spin $a$ and the quadrupolar correction parameter $\epsilon$. In all cases, the impact parameter of the photon is set to $b=1$. The higher the spin, the higher the redshift. Increasing the values of the parameter $\epsilon$ decreases the redshift.

termine the flux ratios between the approaching and receding parts of accretion flow images, as well as the width of relativistically broadened iron lines.

I calculate the redshift at infinity of a photon that is emitted by a particle on the ISCO. The coordinate velocity of a massive particle on a circular orbit in the equatorial plane with radius $r$ is given by (Glampedakis \& Babak 2006)

$$
\begin{gathered}
u^{\mathrm{t}}=\frac{1}{\Delta}\left[E\left(r^{2}+a^{2}\right)+\frac{2 M a}{r}\left(a E-L_{\mathrm{z}}\right)\right]-\epsilon\left(1-\frac{2 M}{r}\right)^{-1} f_{3}(r) E \\
u^{\phi}=\frac{1}{\Delta}\left[\frac{2 M}{r}\left(a E-L_{\mathrm{z}}\right)+L_{\mathrm{z}}\right]-\epsilon \frac{h_{3}(r)}{r^{2}} L_{\mathrm{z}},
\end{gathered}
$$

where

$$
f_{3}(r)=-\frac{5(r-M)}{8 M r(r-2 M)}\left(2 M^{2}+6 M r-3 r^{2}\right)-\frac{15 r(r-2 M)}{16 M^{2}} \ln \left(\frac{r}{r-2 M}\right)
$$




$$
h_{3}(r)=\frac{5}{8 M r}\left(2 M^{2}-3 M r-3 r^{2}\right)+\frac{15}{16 M^{2}}\left(r^{2}-2 M^{2}\right) \ln \left(\frac{r}{r-2 M}\right) .
$$

The orbital energy $E$ and angular momentum about the $z$-axis $L_{\mathrm{z}}$ are given by equations (2.23) and (2.24) and have been normalized to the particle's rest mass $\mu$. In these expressions, the radius is evaluated at $r=r_{\mathrm{ISCO}}$.

The redshift of a photon at infinity is defined by (e.g., Cunningham 1975)

$$
\frac{1}{1+z}=\frac{p_{\mathrm{t}}}{p_{\mathrm{e}} \cdot u}
$$

which includes both the gravitational redshift and the Doppler shift. Here, $p_{\mathrm{t}}$ is the photon energy with respect to an observer at rest at infinity, $p_{\mathrm{e}}$ the 4 momentum of the photon at its emission, and $u$ is the 4-velocity of the emitting particle given by equation (2.27). Therefore, the redshift is equal to

$$
z=u^{\mathrm{t}}-b u^{\phi}-1
$$

where

$$
b=-\frac{p_{\phi}}{p_{\mathrm{t}}}
$$

is the impact parameter of the emitted photon.

Figure 2.6 shows contours of constant redshift $z$ at infinity of a photon with a nominal value of the impact parameter $b=1$ emitted by a particle on the (direct) ISCO around the black hole. The respective values of the redshift range from $z=0.28$ for the line in the top left corner to $z=0.40$ along the line in the bottom right corner in steps of 0.03 . The higher the spin of the black hole, the larger the redshift of the photon, because the radius $r_{\mathrm{ISCO}}$ decreases so that it costs the photon more energy to escape the gravitational attraction of the black hole. For increasing quadrupolar correction $\epsilon$ at a given spin $a$, however, the redshift decreases, because the radius $r_{\text {ISCO }}$ (c.f. Figure 2.3) and the azimuthal velocity $u_{\phi}$ (as well as $u_{\mathrm{t}}$ ) increase. 


\subsubsection{The Circular Photon Orbit}

In principle, I can obtain the radius of the circular photon orbit by inverting expressions (2.23) and (2.24). Once properly expanded to the quadrupole order, the photon radius occurs where the resulting expressions vanish. Unfortunately, in my case, this procedure leads to slightly different results when either the expression for the energy (2.23) or the one for the axial angular momentum (2.24) are inverted. This is a consequence of the expansion to first order in the parameter $\epsilon$ and higher order corrections are required. Instead, I obtain a numerical solution for the circular photon orbit in the following way.

In order to calculate the radius of the circular photon orbit numerically, I solved the geodesic equation (2.15) in the equatorial plane for a photon with a purely azimuthal initial 3-velocity for various initial radii and determined the initial radius for which the photon trajectory changed from being unbound to being bound. In Figure 2.7 I plot the radius of the circular photon orbit $r_{\text {photon }}$ as a function of the spin $a$ for several values of the parameter $\epsilon$. The radius $r_{\text {photon }}$ decreases for increasing values of the spin due to frame dragging, while $r_{\text {photon }}$ increases for increasing values of the parameter $\epsilon$. 


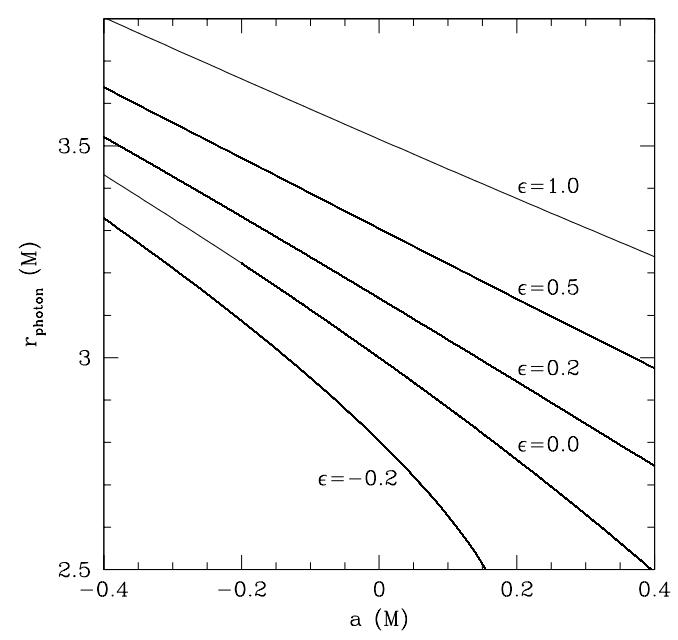

Figure 2.7 The radius of the circular photon orbit $r_{\text {photon }}$ as a function of the spin $a$ for several values of the quadrupolar correction parameter $\epsilon$. The radius $r_{\text {photon }}$ decreases for increasing values of the spin due to frame dragging, while $r_{\text {photon }}$ increases for increasing values of the parameter $\epsilon$.

\subsection{Conclusions}

In Part I of this thesis, I investigate a framework for testing the no-hair theorem with observations of black holes in the electromagnetic spectrum. My framework can be viewed as either a null-hypothesis test of the no-hair theorem within general relativity or as a self-consistent test of general relativity itself in the strongfield regime. If the multipole moments of a black-hole candidate are measured to be different from the moments of the Kerr metric, there are two possibilities. If general relativity is assumed, the astrophysical object is not a black hole. But if it is known to possess a horizon, then both the no-hair theorem and general relativity are incorrect. If the measured multipole moments coincide with the Kerr multipole moments, general relativity and the no-hair theorem may be correct or 
not. A definite answer from the extraction of the multipole moments alone is not possible, because other theories of gravity likewise predict the Kerr metric as a black-hole spacetime (Psaltis et al. 2008).

In this chapter, I formulated my tests based on the quasi-Kerr metric (Glampedakis \& Babak 2006) which contains an independent quadrupole moment and deviates smoothly from the Kerr metric at the quadrupole order. Since the no-hair theorem admits exactly two independent multipole moments, a measurement of three moments allows us to test the no-hair theorem (Ryan 1995). Generalrelativistic black holes must have the multipole structure of the Kerr metric. If a different set of multipoles is detected, then the compact object cannot be a black hole within general relativity.

I estimated the range of validity of the quasi-Kerr metric and demonstrated the dependence of various properties of this spacetime on both the spin and the quadrupole moment of the black hole. I analyzed in detail the effects of light bending, photon redshift, and the respective locations of the ISCO and the circular photon orbit, all of which are of critical importance for observables of accretion flows around astrophysical black holes. In particular, I showed that the radius of the ISCO and the amount of gravitational lensing experienced by photons are altered significantly for already moderate changes of the quadrupole moment.

I identified several observational approaches within my framework that will test the no-hair theorem within the next few years. Among these are imaging observations of accretion flows around black holes and, especially, Sgr A* using VLBI techniques, as well as the precise measurements of the spectra of iron lines and accretion disks from AGN with future X-ray missions such as Astro- $H$ or ATHENA. In the following chapters I will explore in detail the prospects of testing 
the no-hair theorem with such observations. 


\section{CHAPTER 3}

Systematic Study of Pathologies of Black Hole Metrics that

ViOLATE THE NO-HAIR THEOREM

In this chapter, I analyze four parametric deviations from the Kerr metric. I consider the quasi-Kerr metric of Glampedakis \& Babak (QK; Glampedakis \& Babak 2006), the bumpy Kerr metric of Vigeland \& Hughes (BK; Collins \& Hughes 2004; Vigeland \& Hughes 2010), the Manko-Novikov metric (MN; Manko \& Novikov 1992), and the modified-gravity, bumpy Kerr metric of Vigeland, Yunes, \& Stein (MGBK; Vigeland et al. 2011). I aim to identify the manner in which these four metrics violate the no-hair theorem and determine their respective ranges of applicability for observational tests of the no-hair theorem as a function of spin and the strength of the deviation. The contents of this chapter are to be submitted to Physical Review D as Johannsen et al. (2012b). 


\subsection{Parametric Deviations from the Kerr Metric}

Some of the metric deformations that I study in this chapter have been designed as small perturbations of the Kerr metric, which are of the form

$$
g_{\mu \nu}=g_{\mu \nu}^{\mathrm{K}}+\zeta h_{\mu \nu}
$$

where $\zeta$ is an order-counting parameter that reminds me that the metric deformation $h_{\mu \nu}$ is supposed to be small relative to the Kerr metric $g_{\mu \nu}^{\mathrm{K}}$. These include the $\mathrm{QK}, \mathrm{BK}$, and MGBK metrics. Other parametric deviations from the Kerr metric need not be perturbative, which include the MN metric.

\subsubsection{The Quasi-Kerr Metric}

The QK metric derives from the Hartle-Thorne metric (Hartle 1967; Hartle \& Thorne 1968), which was originally designed for slowly rotating neutron stars. This metric contains a quadrupole moment that is independent of both mass and spin.

The QK metric modifies the quadrupole moment of the Kerr metric by the amount

$$
\delta Q_{\mathrm{QK}}=-\epsilon_{\mathrm{QK}} M^{3}
$$

where the parameter $\epsilon_{\mathrm{QK}}$ is a measure for a potential deviation from the Kerr metric quadrupole, so that the full quadrupole moment is of the form (2.10).

In Boyer-Lindquist-like coordinates, the QK metric $g_{\mu \nu}^{\mathrm{QK}}$ is given by Eq. (3.1) with $\zeta \equiv \epsilon_{\mathrm{QK}}$ and (Glampedakis \& Babak 2006)

$$
\begin{aligned}
h_{\mathrm{QK}}^{t t} & =(1-2 M / r)^{-1}\left[\left(1-3 \cos ^{2} \theta\right) \mathcal{F}_{1}(r)\right], \\
h_{\mathrm{QK}}^{r r} & =(1-2 M / r)\left[\left(1-3 \cos ^{2} \theta\right) \mathcal{F}_{1}(r)\right], \\
h_{\mathrm{QK}}^{\theta \theta} & =-\frac{1}{r^{2}}\left[\left(1-3 \cos ^{2} \theta\right) \mathcal{F}_{2}(r)\right],
\end{aligned}
$$




$$
h_{\mathrm{OK}}^{\phi \phi}=-\frac{1}{r^{2} \sin ^{2} \theta}\left[\left(1-3 \cos ^{2} \theta\right) \mathcal{F}_{2}(r)\right],
$$

and $h_{\mathrm{QK}}^{t \phi}=0$. The functions $\mathcal{F}_{1,2}(r)$ are given in Appendix A of Glampedakis \& Babak (2006). In its full form, the quasi-Kerr metric is appropriate only for slowly rotating black holes with values of the spin $a \lesssim 0.4 M$, while it is formally correct up to the maximum value of the spin if $\epsilon_{\mathrm{QK}}=0$.

\subsubsection{The Bumpy Kerr Metric}

The BK metric modifies the Kerr spacetime through small perturbations due to external stresses. Collins \& Hughes (2004) defined mass perturbations by starting from the most general stationary and spherically-symmetric metric in Weyl form

$$
d s_{\mathrm{W}}^{2}=-e^{2 \psi} d t^{2}+e^{2 \gamma-2 \psi}\left(d \rho^{2}+d z^{2}\right)+e^{-2 \psi} \rho^{2} d \phi^{2} .
$$

They defined $\psi \equiv \psi_{0}+\zeta_{\mathrm{BK}} \psi_{1}$ and $\gamma \equiv \gamma_{0}+\zeta_{\mathrm{BK}} \gamma_{1}$, with $\left(\psi_{0}, \gamma_{0}\right)$ equal to the Schwarzschild values, $\left(\psi_{1}, \gamma_{1}\right)$ parametric deformations, and $\zeta_{\text {Вк }} \ll 1$ an ordercounting parameter. They then required this metric to satisfy the Einstein equations to linear order in $\zeta_{\mathrm{BK}}$, thus obtaining differential equations for the deformation functions.

This was generalized to a spinning spacetime by applying a Newman-Janis rotation (Vigeland \& Hughes 2010). In Boyer-Lindquist-like coordinates, the BK metric is given by Eq. (3.1) with (Vigeland \& Hughes 2010)

$$
\begin{aligned}
h_{t t}^{\mathrm{BK}} & =-2\left(1-\frac{2 M r}{\Sigma}\right) \psi_{1}, \\
h_{t r}^{\mathrm{BK}} & =-\gamma_{1} \frac{2 a^{2} M r \sin ^{2} \theta}{\Delta \Sigma}, \\
h_{t \phi}^{\mathrm{BK}} & =\left(\gamma_{1}-2 \psi_{1}\right) \frac{2 a M r \sin ^{2} \theta}{\Sigma}, \\
h_{r r}^{\mathrm{BK}} & =2\left(\gamma_{1}-\psi_{1}\right) \frac{\Sigma}{\Delta},
\end{aligned}
$$




$$
\begin{aligned}
h_{r \phi}^{\mathrm{BK}} & =\gamma_{1}\left[1+\frac{2 M r\left(r^{2}+a^{2}\right)}{\Delta \Sigma}\right] a \sin ^{2} \theta \\
h_{\theta \theta}^{\mathrm{BK}} & =2\left(\gamma_{1}-\psi_{1}\right) \Sigma \\
h_{\phi \phi}^{\mathrm{BK}} & =\left[\left(\gamma_{1}-\psi_{1}\right) \frac{8 a^{2} M^{2} r^{2} \sin ^{2} \theta}{\Delta \Sigma(\Sigma-2 M r)}-2 \psi_{1}\left(1-\frac{2 M r}{\Sigma}\right)^{-1}\right] \Delta \sin ^{2} \theta .
\end{aligned}
$$

The perturbations $\psi_{1}$ and $\gamma_{1}$ satisfy linearized Einstein equations, which can be solved through a multipolar decomposition. At lowest order $(\ell=2)$, these functions are given by (Vigeland \& Hughes 2010)

$$
\begin{aligned}
\psi_{1}^{\ell=2}(r, \theta)= & \frac{B_{2} M^{3}}{4} \sqrt{\frac{5}{\pi}} \frac{1}{d(r, \theta, a)^{3}}\left[\frac{3 L(r, \theta, a)^{2} \cos ^{2} \theta}{d(r, \theta, a)^{2}}-1\right] \\
\gamma_{1}^{\ell=2}(r, \theta)= & B_{2} \sqrt{\frac{5}{\pi}} \\
& \times\left[\frac{L(r, \theta, a)}{2} \frac{c_{20}(r, a)+c_{22}(r, a) \cos ^{2} \theta+c_{24}(r, a) \cos ^{4} \theta}{d(r, \theta, a)^{5}}-1\right]
\end{aligned}
$$

where

$$
\begin{aligned}
d(r, \theta, a) & =\sqrt{r^{2}-2 M r+\left(M^{2}+a^{2}\right) \cos ^{2} \theta} \\
L(r, \theta, a) & =\sqrt{(r-M)^{2}+a^{2} \cos ^{2} \theta} \\
c_{20}(r, a) & =2(r-M)^{4}-5 M^{2}(r-M)^{2}+3 M^{4}, \\
c_{22}(r, a) & =5 M^{2}(r-M)^{2}-3 M^{4}+a^{2}\left[4(r-M)^{2}-5 M^{2}\right] \\
c_{24}(r, a) & =a^{2}\left(2 a^{2}+5 M^{2}\right) .
\end{aligned}
$$

The strength of the perturbation to the Kerr metric at this order is determined by the parameter $B_{2}$.

Vigeland (2011) showed that at lowest order, this perturbation changes the mass quadrupole, which is given by

$$
Q_{\mathrm{BK}}=-M\left(a^{2}+\frac{1}{2} B_{2} M^{2} \sqrt{\frac{5}{\pi}}\right),
$$


so that

$$
\delta Q_{\mathrm{BK}}=-\frac{1}{2} B_{2} M^{3} \sqrt{\frac{5}{\pi}} .
$$

This equation suggests a formal relationship between the deformation parameter $\epsilon_{\mathrm{QK}}$ of the QK metric and the parameter $B_{2}$ of the BK metric via the relation

$$
\epsilon_{\mathrm{QK}}=\frac{1}{2} B_{2} \sqrt{\frac{5}{\pi}} \approx 0.63 B_{2} .
$$

This mapping is somewhat misleading, however, as one might be tempted to conclude that the quasi-Kerr and bumpy Kerr parameterizations are equivalent when in fact they are not. The respective perturbations of the Kerr metric in the quasi-Kerr and bumpy Kerr metrics are different due to the different functional forms of the corrections $h_{\mu \nu}$ and $b_{\mu \nu}$ given by the expressions (3.3) and (3.7), respectively. In addition, for nonzero deviations from the Kerr metric, the quasiKerr metric is a solution of the Einstein equations only up to the quadrupole order, while the bumpy Kerr metric is a solution of the linearized Einstein equations to all multipole orders $l$.

\subsubsection{The Ricci flat metric proposed by Manko \& Novikov}

The MN metric is a non-linear superposition of the Kerr metric with a static vacuum field that generalizes the former to a Ricci-flat spacetime with arbitrary mass and current multipole moments. In its original form (Manko \& Novikov 1992), this metric harbors a naked singularity surrounded by an event horizon in the equatorial plane (Manko \& Novikov 1992). Gair et al. (2008) and Brink (2008) analyzed properties of orbits in the Manko-Novikov spacetime and found regions near the central singularity where geodesic motion becomes ergodic. They identified domains containing closed timelike curves around the origin which, therefore violate causality. In addition, Gair et al. (2008) computed the location 
of the ISCO and the orbital frequencies. Berti et al. (2005) analyzed the MankoNovikov metric in the context of rotating neutron stars, and Bambi \& Barausse (2011) studied thermal emission spectra of accretion disks in a Manko-Novikov background. Ergodic orbits in general non-Kerr spacetimes were analyzed by Apostolatos et al. (2009) and Gerakopoulos et al. (2010).

In this chapter, I will use a subclass of the MN metrics that describes electrically neutral compact objects and that is given by the line element of (Manko \& Novikov 1992) transformed to spherical coordinates

$$
\begin{aligned}
d s_{\mathrm{MN}}^{2}= & -f d t^{2}+2 f \omega d t d \phi+\frac{e^{2 \gamma}}{f} \frac{(r-M)^{2}-\left(M^{2}-a^{2}\right) \cos ^{2} \theta}{\Delta} d r^{2} \\
& +\frac{e^{2 \gamma}}{f}\left[(r-M)^{2}-\left(M^{2}-a^{2}\right) \cos ^{2} \theta\right] d \theta^{2} \\
& +f^{-1}\left(\Delta \sin ^{2} \theta-f^{2} \omega^{2}\right) d \phi^{2},
\end{aligned}
$$

with $f=f(r, \theta), \omega=\omega(r, \theta)$. The quantities $\gamma, f$ and $\omega$ are given in Appendix A.

Following Gair et al. (2008), I define

$$
q_{\mathrm{MN}} \equiv \zeta_{\mathrm{MN}} \equiv-\frac{M_{2}-M_{2}^{\mathrm{K}}}{M^{3}},
$$

which is a dimensionless parameter that measures the $\mathrm{MN}$ deviation from the Kerr quadrupole moment $M_{2}^{\mathrm{K}}$. The first few non-vanishing multipole moments are given by Manko \& Novikov (1992) (but see Gair et al. (2008) and references therein)

$$
\begin{aligned}
& M_{0}=M, \quad S_{1}=a M, \\
& M_{2}=-M\left(a^{2}+q_{\mathrm{MN}} M^{2}\right), \\
& S_{3}=-a M\left(a^{2}+2 q_{\mathrm{MN}} M^{2}\right) .
\end{aligned}
$$




\subsubsection{The Modified Gravity Bumpy Kerr Metric}

The MGBK metric, proposed by Vigeland, Yunes, and Stein, deforms the Kerr metric through certain bump functions, such that the resulting metric possesses four constants of the motion. Such a metric is also not Ricci flat. Vigeland, Yunes, and Stein (Vigeland et al. 2011) have shown that specific choices of the bump functions reproduce all known modified-gravity black hole solutions known to date.

In this chapter, I use the class of MGBK metrics studied in Gair et al. (2008), where certain simplifications are made to guarantee certain properties (see Section IIB in Gair \& Yunes 2011). With this at hand, the non-vanishing components of MGBK metric in Boyer-Lindquist-like coordinates are as in Eq. (3.1) with 


$$
\begin{aligned}
& h_{t t}^{\mathrm{MGBK}}=-\frac{a}{M} \frac{P_{2}}{P_{1}} h_{t \phi}^{\mathrm{MGBK}}-\frac{a}{2 M} \frac{\Sigma^{2} \Delta}{P_{1}} \frac{\partial h_{t \phi}^{\mathrm{MGBK}}}{\partial r}+\frac{\left(r^{2}+a^{2}\right) \hat{\rho}^{2} \Delta}{P_{1}} \gamma_{1} \\
& +\frac{2 a^{2} r^{2} \Delta \sin ^{2} \theta}{P_{1}} \gamma_{1}-\frac{a}{M} \frac{\Delta \sin ^{2} \theta}{\Sigma} \frac{P_{3}}{P_{1}} \gamma_{3}+\frac{2 \Delta}{\Sigma} \frac{P_{4}}{P_{1}} \gamma_{4} \\
& -\frac{a^{2}}{2 M} \frac{\Sigma \Delta^{2} \sin ^{2} \theta}{P_{1}} \frac{d \gamma_{1}}{d r}-\frac{a}{2 M} \frac{\Delta^{2}\left(\hat{\Sigma}+2 a^{2} M r \sin ^{2} \theta\right) \sin ^{2} \theta}{P_{1}} \frac{d \gamma_{3}}{d r} \\
& -\frac{a^{2}}{2 M} \frac{\Delta^{2}(\Sigma-4 M r) \sin ^{2} \theta}{P_{1}} \frac{d \gamma_{4}}{d r} \\
& h_{r r}^{\mathrm{MGBK}}=-\frac{\Sigma \gamma_{1}}{\Delta}, \\
& h_{\phi \phi}^{\mathrm{MGBK}}=-\frac{\left(r^{2}+a^{2}\right)^{2}}{a^{2}} h_{t t}^{\mathrm{MGBK}}+\frac{\Delta}{a^{2}} \Sigma \gamma_{1}-\frac{2\left(r^{2}+a^{2}\right)}{a} h_{t \phi}^{\mathrm{MGBK}}-\frac{2 \Delta^{2} \sin ^{2} \theta}{a} \gamma_{3} \\
& +\frac{2 \Delta^{2}}{a^{2}} \gamma_{4} \\
& \frac{\partial^{2} h_{t \phi}^{\mathrm{MGBK}}}{\partial r^{2}}=\frac{2 a^{2} \sin ^{2} \theta}{\Sigma^{2}} \frac{P_{6}}{P_{1}} h_{t \phi}^{\mathrm{MGBK}}-\frac{2 r}{\Sigma} \frac{P_{7}}{P_{1}} h_{t \phi}^{\mathrm{MGBK}}+\frac{4 a M r \sin ^{2} \theta}{\Sigma} \frac{P_{15}}{P_{16}} \gamma_{1} \\
& -\frac{4 a M r \sin ^{2} \theta}{\Sigma^{2}} \frac{P_{8}}{P_{1}} \gamma_{1}+\frac{2 \sin ^{2} \theta}{\Sigma^{2}} \frac{P_{10}}{P_{1}} \gamma_{3}-\frac{16 a M \sin ^{2} \theta}{\Sigma^{2}} \frac{P_{11}}{P_{1}} \gamma_{4} \\
& -\frac{2 a}{\Sigma^{2}} \frac{P_{12}}{P_{1}} \frac{d \gamma_{1}}{d r}-\frac{2 \sin ^{2} \theta}{\Sigma^{2}} \frac{P_{13}}{P_{1}} \frac{d \gamma_{3}}{d r}-\frac{2 a \sin ^{2} \theta}{\Sigma^{2}} \frac{P_{14}}{P_{1}} \frac{d \gamma_{4}}{d r} \\
& -\frac{a \Delta \sin ^{2} \theta}{\Sigma} \frac{d^{2} \gamma_{1}}{d r^{2}}-\frac{\Delta \sin ^{2} \theta}{\Sigma^{2}}\left(\hat{\Sigma}+2 a^{2} M r \sin ^{2} \theta\right) \frac{d^{2} \gamma_{3}}{d r^{2}} \\
& -\frac{a \Delta(\Sigma-4 M r) \sin ^{2} \theta}{\Sigma^{2}} \frac{d^{2} \gamma_{4}}{d r^{2}}
\end{aligned}
$$

where

$$
\begin{aligned}
& \hat{\rho}^{2} \equiv r^{2}-a^{2} \cos ^{2} \theta, \quad f \equiv 1-\frac{2 M}{r} \\
& \hat{\Sigma} \equiv\left(r^{2}+a^{2}\right)^{2}-a^{2} \Delta \sin ^{2} \theta .
\end{aligned}
$$

and $P_{i}$ are polynomials in $(r, \cos \theta)$, given in the appendix of Vigeland et al. (2011) (I here adopt the deformed Kerr parameterization of Vigeland et al. 2011).

The bumpy functions $\gamma_{i}=\gamma_{i}(r)$ depend on radius, and I parameterize this 
dependence via Gair \& Yunes (2011)

$$
\begin{aligned}
\gamma_{A} & =\sum_{n=0}^{\infty} \gamma_{A, n}\left(\frac{M}{r}\right)^{n}, \\
\gamma_{3} & =\frac{1}{r} \sum_{n=0}^{\infty} \gamma_{3, n}\left(\frac{M}{r}\right)^{n},
\end{aligned}
$$

where $A$ is 1 or 4 and $\left(\gamma_{1, n}, \gamma_{3, n}, \gamma_{4, n}\right)$ are constants that control the magnitude of the deformations. Additional simplifications (Gair \& Yunes 2011) allow me to set $\gamma_{1,0}=\gamma_{1,1}=\gamma_{3,0}=\gamma_{4,0}=\gamma_{4,1}=\gamma_{3,2}=0$.

The metric components $h_{t t}^{\mathrm{MGBK}}$ and $h_{\phi \phi}^{\mathrm{MGBK}}$ are fully determined once I solve the differential equation (3.16) for $h_{t \phi}$. Doing so in a far-field expansion, I find

$$
h_{t \phi}^{\mathrm{MGBK}}=M \sum_{n=2}^{N} h_{t \phi, n}(\theta)\left(\frac{M^{n}}{\Sigma^{n / 2}}\right),
$$

where the coefficients $h_{t \phi, n}$ are given in Gair \& Yunes (2011). Notice that the expressions presented here defer slightly from that of Gair \& Yunes (2011), as I use here a dimensional Kerr spin parameter $a$ and define $\Sigma$ and $\rho$ differently than in Gair \& Yunes (2011). 


\subsection{Properties of the Spacetimes}

Due to the no-hair theorem, each of the metrics discussed in Section 3.1 has to violate at least one of its requirements. In the following, I analyze the properties of these metrics in this regard and demonstrate explicitly how they violate the no-hair theorem. In addition, I estimate the respective ranges of applicability of these metrics to be used for astrophysical tests of the no-hair theorem.

\subsubsection{Conserved Quantities and Petrov Type}

By construction, all the metrics considered in Section 3.1 admit two Killing vectors corresponding to stationarity and axisymmetry. This implies that these spacetimes possess an associated conserved energy and (z-component of) angular momentum as well as the conserved rest mass of a test particle. The MGBK metric possesses in addition an approximately conserved fourth quantity (a Carter constant), associated with the existence of an approximate Killing tensor (approximate in the sense that it satisfies Killing's equation to linear order in the deformation parameters). Therefore, the MGBK metric is of approximate Petrov type $\mathrm{D}$, while the other four metrics $(\mathrm{QK}, \mathrm{MN}, \mathrm{BK})$ are in general of Petrov type I.

Some metrics considered here satisfy the vacuum Einstein equations, while others do not. The MN metric is Ricci-flat, and thus, is a solution of the vacuum Einstein equations. The QK metric is Ricci-flat only up to the quadrupole order (i.e., neglecting terms of order $\epsilon_{\mathrm{QK}} a, \epsilon_{\mathrm{QK}}^{2}$ ). The $\mathrm{BK}$ metric is a vacuum solution only of the linearized Einstein equations in the limit $a \rightarrow 0$, i.e., the Ricci tensor contains terms of order $a B_{2}$ Vigeland \& Hughes (2010). The MGBK metric does not satisfy the vacuum Einstein equations and is not Ricci flat. 


\subsubsection{Asymptotic Flatness}

The spacetime of a black hole in isolation must be asymptotically flat, i.e., there exists a coordinate system $\left(x^{0}, x^{1}, x^{2}, x^{3}\right)$ such that all metric components in these coordinates behave as $g_{\mu \nu}=\eta_{\mu \nu}+\mathcal{O}(1 / r)$ as $r \rightarrow \infty$ in either spatial or null directions, where $r$ is the Euclidean norm of the spatial coordinates (Beig 1980; Beig \& Simon 1981). In terms of the line element, the subleading terms must fall-off as (e.g., Heusler 1996):

$$
\begin{aligned}
d s^{2}= & -\left[1-\frac{2 M}{r}+\mathcal{O}\left(r^{-2}\right)\right] d t^{2}-\left[\frac{4 M a}{r} \sin ^{2} \theta+\mathcal{O}\left(r^{-2}\right)\right] d t d \phi \\
& +\left[1+\mathcal{O}\left(r^{-1}\right)\right]\left[d r^{2}+r^{2}\left(d \theta^{2}+\sin ^{2} \theta d \phi^{2}\right)\right]
\end{aligned}
$$

in Boyer-Lindquist-like coordinates. Asymptotically flat spacetimes with a slower fall-off cannot be stationary in general relativity (Kennefick \& Ó Murchadha 1995). The above definitions of asymptotic flatness are not precise, due to issues with coordinate invariance and the precise way in which the limit $r \rightarrow \infty$ is taken (see Wald 1984 for further details). However, this definition will suffice for my purposes in this chapter.

For all of the metrics that I analyze in this chapter, the Kerr part is clearly asymptotically flat; I are thus left with the task of showing that the deviations of these metrics from the Kerr metric do not spoil the asymptotic flatness of the background. The asymptotic flatness of the BK and MGBK metrics has already been shown Gair \& Yunes (2011). I now turn to the QK and MN metrics.

As an example of the QK metric, I consider the $(t, t)$ component of the QK metric, which has the form

$$
h_{t t}^{\mathrm{QK}} \propto \frac{2 M}{r^{2}}\left(2 M^{3}+4 M^{2} r-9 M r^{2}+3 r^{3}\right)-3(r-2 M)^{2} \ln \left(\frac{r}{r-2 M}\right) .
$$


One can expand the logarithm in $r \gg M$ to show that $h_{t t}^{\mathrm{OK}} \propto-(16 / 5) M^{5} / r^{3}$, which clearly remains asymptotically flat. A similar argument holds for the other components of the QK metric.

The MN metric should be considered separately, as here its asymptotic flatness is not as clear. To see its structure more clearly, let me perform the coordinate transformation

$$
r^{\prime} \equiv \exp \left[\frac{4 q_{\mathrm{MN}}}{\left(1-\chi^{2}\right)^{3 / 2}}\right] r, \quad \phi^{\prime} \equiv \exp \left[-\frac{4 q_{\mathrm{MN}}}{\left(1-\chi^{2}\right)^{3 / 2}}\right] \phi,
$$

with $(t, \theta)$ unchanged and $\chi \equiv a / M$. This transformation ensures that the MN metric reduces to Minkowski spacetime at radial infinity. Transforming the metric, expanding its elements in $M / r \ll 1$ and linearizing them in $q_{\mathrm{MN}} \ll 1$, the metric perturbation becomes (dropping primes)

$$
\begin{aligned}
h_{t t} & =-\frac{8 q_{\mathrm{MN}} M}{\left(1-\chi^{2}\right)^{3 / 2}} \frac{1}{r}+\mathcal{O}\left(r^{-2}\right) \\
h_{t \phi} & =\frac{32 \chi q_{\mathrm{MN}} M^{2} \sin ^{2} \theta}{\left(1-\chi^{2}\right)^{3 / 2}} \frac{1}{r}+\mathcal{O}\left(r^{-2}\right) \\
h_{r r} & =-\frac{8 q_{\mathrm{MN}} M}{\left(1-\chi^{2}\right)^{3 / 2}} \frac{1}{r}-\frac{8 q_{\mathrm{MN}} M^{2}\left(4-\chi^{2} \sin ^{2} \theta\right)}{\left(1-\chi^{2}\right)^{3 / 2}} \frac{1}{r^{2}}+\mathcal{O}\left(r^{-3}\right) \\
h_{\theta \theta} & =-\frac{8 q_{\mathrm{MN}} \chi^{2} M^{2} \cos ^{2} \theta}{\left(1-\chi^{2}\right)^{3 / 2}}+\mathcal{O}\left(r^{-1}\right) \\
h_{\phi \phi} & =-\frac{8 q_{\mathrm{MN}} \chi^{2} M^{2} \sin ^{2} \theta}{\left(1-\chi^{2}\right)^{3 / 2}}+\mathcal{O}\left(r^{-3}\right) .
\end{aligned}
$$

The parameter $M$ has to be rescaled in order for the MN metric to describe the correct Newtonian limit in the non-relativistic regime:

$$
M^{\prime} \equiv M \exp \left[-\frac{4 q_{\mathrm{MN}}}{\left(1-\chi^{2}\right)^{3 / 2}}\right] .
$$

This then eliminates all components of the metric perturbation to relative $\mathcal{O}\left(r^{-3}\right)$, with the deformed metric equal to the Kerr one with parameters $M^{\prime}$ and $a^{\prime}=\chi M^{\prime}$, 
and shows that the MN metric is indeed asymptotically flat. An investigation of a rescaling of the full metric and its parameters at $\mathcal{O}\left(r^{-3}, q_{\mathrm{MN}}^{2}\right)$ is beyond the scope of my analysis. From here on, I will use the MN metric in the form given by Eq. (3.13) after applying the coordinate transformation in Eqs. (3.24) and the rescaling in Eq. (3.26).

\subsubsection{Singularities and the Event Horizon}

In this section, I analyze the location of the event horizon, the nature of the central singularity, and the potential presence of coordinate singularities in the exterior domain of the metrics presented in Section 3.1.

I begin by defining the event horizon as the outermost apparent horizon, where the latter is the 2-surface where the expansion of a congruence of outgoing null geodesics orthogonal to the 2-surface vanishes.

For any stationary and asymptotically flat spacetime, the event horizon is also a Killing horizon for some Killing vector $\chi^{\mu}$ if Hawking's rigidity theorem (Hawking 1972) or an appropriate generalization thereof (see, e.g., Friedrich et al. 1999) can be applied. Then, this Killing vector can be written as $\chi^{\mu}=t^{\mu}+\Omega \phi^{\mu}$ for some constant $\Omega$, where $t^{\mu}$ and $\phi^{\mu}$ are the temporal and azimuthal Killing vectors of the spacetime (see, e.g., Carroll 2004). Requiring that this vector be null forces the condition

$$
g_{\phi \phi}\left(\Omega^{2}+2 \Omega \frac{g_{t \phi}}{g_{\phi \phi}}+\frac{g_{t t}}{g_{\phi \phi}}\right)=0,
$$

which I can use to solve for the constant

$$
\Omega_{ \pm}=-\frac{g_{t \phi}}{g_{\phi \phi}} \pm \sqrt{\frac{g_{t \phi}^{2}}{g_{\phi \phi}^{2}}-\frac{g_{t t}}{g_{\phi \phi}}} .
$$

As one approaches the event horizon, the angular velocity $\Omega_{ \pm}$must approach a constant, $\Omega_{ \pm} \rightarrow-g_{t \phi} / g_{\phi \phi}$. This is because this constant represents the angu- 
lar velocity of zero-angular momentum observers at the horizon, which must be single-valued. The only way this can happen is if (see, e.g., Poisson 2004)

$$
g_{t \phi}^{2}-g_{t t} g_{\phi \phi}=0
$$

The radius at which Eq. 3.29 is satisfied defines the Killing horizon for the spacetime, since there $\chi^{\mu}$ is null.

In general, however, the Killing and event horizons of a stationary spacetime are distinct (Poisson 2004). Since the event horizon is an apparent horizon, it is a null, stationary surface. The normal to such a surface is proportional to $\partial_{\alpha} r$, and the surface is null if (Poisson 2004)

$$
g^{\alpha \beta}\left(\partial_{\alpha} r\right)\left(\partial_{\beta} r\right)=g^{r r}=0
$$

which is not necessarily equivalent to Eq. (3.29). I will use the more general condition (3.30) to compute the location of the event horizons of the spacetime I analyze in this chapter.

If the event horizon of a given metric is entirely closed, the metric describes a black hole. If, on the other hand, the event horizon has holes and is either partly or completely absent, the metric describes a naked singularity. In addition to the event horizon, one might also wish to find the location of possible coordinate singularities, i.e., the coordinate location at which the metric is singular. The Kerr metric in Boyer-Lindquist coordinates has a coordinate singularity at the location of the horizon

$$
r_{\text {Hor }}^{\mathrm{K}}=r_{+}^{\mathrm{K}}=M+\sqrt{M^{2}-a^{2}} .
$$

Of course, this need not be the case; for example, the Kerr metric in Kerr-Schild coordinates is not singular at the location of the event horizon. 


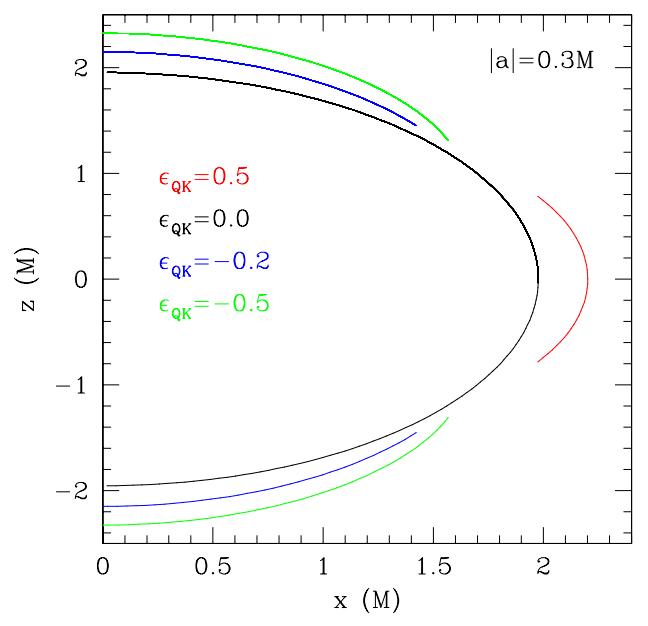

Figure 3.1 Event horizon of the central object in the QK metric with a spin of $|a|=0.3 M$ for several values of the parameter $\epsilon_{\mathrm{QK}}$. If $\epsilon_{\mathrm{QK}} \neq 0$, the horizon is open, and the central object is a naked singularity.

In the $\mathrm{QK}$ metric, the element $g_{\mathrm{QK}}^{r r}$ is given by the expression

$$
\begin{aligned}
g_{\mathrm{QK}}^{r r}= & \frac{\Delta}{\Sigma}+\frac{5 \epsilon_{\mathrm{QK}}}{16 r^{2}}\left(1-3 \cos ^{2} \theta\right) \\
& {\left[2\left(3 r^{3}-9 M r^{2}+4 M^{2} r+2 M^{3}\right)\right.} \\
& \left.-3 r^{2}\left(r^{2}-2 M^{2}\right) \ln \left(\frac{r}{r-2 M}\right)\right] .
\end{aligned}
$$

If $\epsilon_{\mathrm{QK}} \neq 0$, equation (3.30) does not have a solution for all values of the angle $\theta$. In this case, the event horizon is open with holes either near the equatorial plane, around the poles, or in between. The central object then constitutes a naked singularity. For positive values of the parameter $\epsilon_{\mathrm{QK}}$, the horizon has a more oblate shape relative to the horizon of a Kerr black hole of equal spin, while the shape of the horizon is more prolate for negative values of the parameter $\epsilon_{\mathrm{QK}}$. The location of the event horizon in the equatorial plane increases with increasing values 
of the parameter $\epsilon_{\mathrm{QK}}$ as already found in Chapter 2. In Fig. 3.1, I plot for illustrative purposes the QK event horizon for $|a|=0.3 M$ and several values of the parameter $\epsilon_{\mathrm{QK}}$ in the $x z$-plane, where $x \equiv \sqrt{r^{2}+a^{2}} \sin \theta$ and $z \equiv r \cos \theta$.
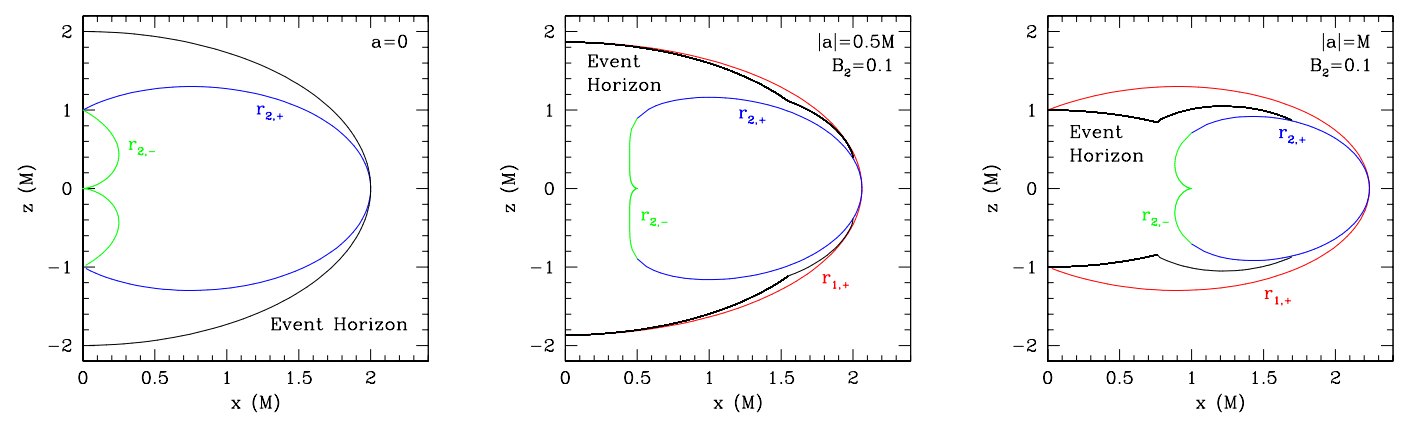

Figure 3.2 Location of the BK event horizon and the coordinate singularities in the $x z$-plane for $a=0,|a|=0.5 M$, and $|a|=M$. For $a=0$ and $0<\theta<\pi / 2$, the coordinate singularity $r_{1,+}$ coincides with the event horizon, and the singularities $r_{2,+}$ and $r_{2,-}$ are located inside the BK event horizon. At $\theta=\pi / 2$, the event horizon has a hole. As $|a|$ increases, the coordinate singularity $r_{1,+}$ moves outside of the BK event horizon. For small but positive values of the spin $|a|$, the event horizon is closed if $B_{2}>0$ and open if $B_{2}<0$. For $|a|>>0$, the event horizon is open around the equatorial plane, and the equatorial hole increases as $|a|$ increases.

If I look at the BK metric in Eq. 3.7 and the definitions of the perturbation functions $\psi_{1}$ and $\gamma_{1}$ in Eq. (3.8), I see that the metric becomes singular at $r_{+}^{\mathrm{K}}$, as well as where $\Sigma-2 M r=0$ and $d(r, \theta, a)=0$. The second condition occurs when $r$ is given by

$$
r_{1, \pm}=M \pm \sqrt{M^{2}-a^{2} \cos ^{2} \theta},
$$

where I note that $r_{1,+}>r_{1,-}$, and $r_{1,+}$ coincides with the location of the Kerr ergosphere. The third condition occurs when $r$ is given by

$$
r_{2, \pm}=M \pm \sqrt{M^{2} \sin ^{2} \theta-a^{2} \cos ^{2} \theta},
$$


where clearly $r_{2,+}>r_{2,-}$. Inside the region bounded by $r_{2,+}$ and $r_{2,-}$, the metric is undefined because $d(r, \theta, a)$ is imaginary.

To leading order in the perturbation, the element $g_{\mathrm{BK}}^{r r}$ is

$$
g_{\mathrm{BK}}^{r r}=\frac{\Delta}{\Sigma}\left[1-2\left(\gamma_{1}-\psi_{1}\right)\right] .
$$

Therefore, as long as the bump functions $\gamma_{1}$ and $\psi_{1}$ remain regular, i.e., as long as $r>r_{2,+}$, the event horizon is either determined by the condition $\Delta=0$ as in the Kerr metric or by the condition $\gamma_{1}-\psi_{1}=1 / 2$.

In Fig. 3.2 I plot the location of the event horizon and the coordinate singularities in the $x z$-plane for $a=0,|a|=0.5 M$, and $|a|=M$. Since $a$ appears in the expressions for $r_{1, \pm}$ and $r_{2, \pm}$ as $a^{2}$, the locations of the coordinate singularities are the same for co- and counter-rotating black holes. For non-spinning black holes, the coordinate singularity $r_{1,+}$ coincides with the BK event horizon, and the coordinate singularity $r_{2,+}$ is hidden inside the BK event horizon at angles $0 \leq \theta<\pi / 2$. In the equatorial plane the event horizon has a hole. As $|a|$ increases, the coordinate singularity $r_{1,+}$ moves outside of the event horizon. For small but nonzero values of the spin $|a|$, the event horizon is closed if $B_{2}>0$ and open near the equatorial plane if $B_{2}<0$ (see Figure 3.4). For $|a|>>0$, the event horizon has a hole around the equatorial plane, which is bound by the coordinate singularity $r_{2,+}$. This equatorial hole increases as $|a|$ increases.

The MN metric in prolate spheroidal coordinates $(t, x, y, \phi)$ harbors a naked singularity with a point-like event horizon located at $x_{\text {Hor }}=1$ (Manko \& Novikov 1992). In Boyer-Lindquist coordinates, however, the event horizon coincides with the Kerr horizon as can be seen from a careful analysis of the metric element $g_{\mathrm{MN}}^{r r}$ which I do not write here explicitly. The event horizon is likewise the location of 
a coordinate singularity.

For the MGBK metric, to leading order in the perturbation, the element $g_{\mathrm{MGBK}}^{r r}$ is

$$
g_{\mathrm{MGBK}}^{r r}=\frac{\Delta}{\Sigma}\left(1+\gamma_{1}\right),
$$

where $\gamma_{1}$ is given by the expression (3.20). Similar to the case of the BK metric, the event horizon is, then, determined by either the condition $\Delta=0$ or the condition $\gamma_{1}=-1$. In particular, if $\gamma_{1, n} \geq 0$ for $n \geq 2$, the event horizon coincides with the Kerr event horizon $r_{\mathrm{Hor}}^{\mathrm{MGBK}}=r_{+}^{\mathrm{K}}$. Moreover, all the components of the metric and the inverse metric become singular only at $r=r_{+}$or at the ring singularity. However, by direct evaluation of quadratic invariants, it is clear that $r=r_{+}$is the location of a coordinate singularity, and not an essential one (the latter remains at the Kerr ring singularity).

\subsubsection{Lorentz Violations, Closed Timelike Curves, and Regions of Validity}

In Subsection 3.2.2, I studied the asymptotic structure of the metrics at spatial infinity, but this does not guarantee that they retain their Lorentzian signatures close to the black holes. When I say that a metric is of Lorentzian signature, what I mean is that

$$
\operatorname{det}\left(g_{\mu \nu}\right)<0
$$

If the metric is Minkowski, then the determinant is simply -1. If the metric is Kerr, the determinant is

$$
\operatorname{det}\left(g_{\mu \nu}^{\mathrm{K}}\right)=-\Sigma^{2} \sin ^{2} \theta
$$

which is clearly negative definite everywhere outside the singularity.

Similarly, let us now study whether the metrics proposed in Section 3.1 remain of Lorentzian signature everywhere outside their event horizons. If this were 
not the case, then the metric would not respect Lorentz symmetry and causality could be broken. What is perhaps worse, since I already showed that the metrics have Lorentzian signature at spatial infinity since they are asymptotically flat, is if their signature changes sign close but outside their event horizons. In that case, their determinants must vanish somewhere outside their horizons, making them singular.

In accordance with the no-hair theorem, the exterior domain of the Kerr metric, i.e., the domain outside the event horizon, is causally well-behaved and free of closed timelike curves if $a \leq M$ (Carter 1968). This follows from the fact that, for constant times $t$, the hypersurfaces $(r, \theta, \phi)$ are always spacelike. This comes about because for constant $(t, \phi)$, the two-dimensional metric induced on surfaces $(r, \theta)$ is positive definite and

$$
\zeta_{\mu}^{(\phi)} \zeta_{(\phi)}^{\mu}=g_{\phi \phi}>0
$$

where $\zeta_{(\phi)}^{\mu}=(0,0,0,1)$ is the axial Killing vector (Carter 1968, 1973). If $|a|>$ $M$, the event horizon disappears and a naked singularity emerges. In this case, causality is violated, because any event in that spacetime can be connected to any other event by both a future and a past directed timelike curve (Carter 1968, 1973; see, also, Clarke \& de Felice 1982).

Closed timelike curves may exist if the metric is no longer Lorentzian or if $\zeta_{\mu} \zeta^{\mu}<0$. In the latter case, e.g., circles with $t=$ const, $r=$ const, $\theta=$ const are closed timelike curves, because the vector $\zeta^{\mu}$ is timelike, which can be thought of as the "tipping over" of light cones. In the Kerr spacetime with $a \leq M$, closed timelike curves exist inside the (inner) event horizon $r_{-}$(Carter 1968). Other examples of spacetimes with closed timelike curves include the van Stockum space- 
time (van Stockum 1937), the Gödel universe (Gödel 1949), and the Gott cosmic string (Gott 1991).
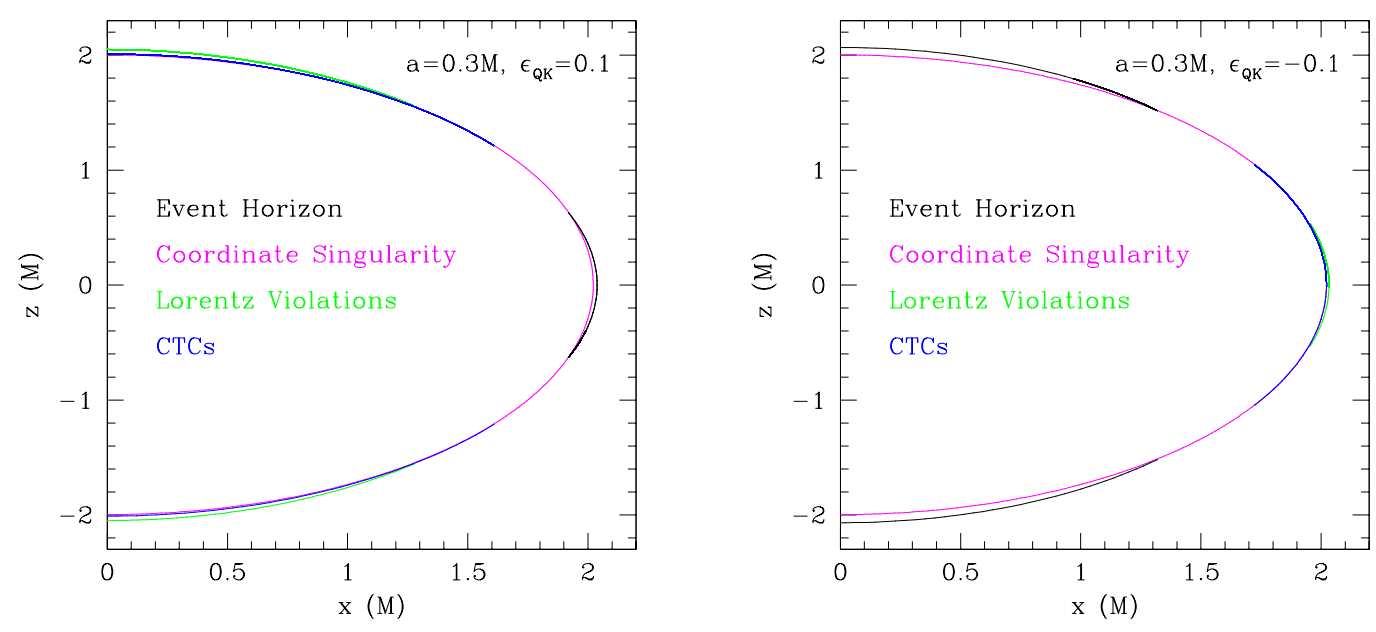

Figure 3.3 Event horizon and regions with Lorentz violations and closed timelike curves in the QK metric for $a=0.3 M$. Top: $\epsilon_{\mathrm{QK}}=0.1$; Lorentz violations and closed timelike curves occur around the poles. Bottom: $\epsilon_{\mathrm{QK}}=-0.1$; Lorentz violations and closed timelike curves occur near the equatorial plane.

The determinant of the quasi-Kerr metric is given by

$$
\begin{aligned}
\operatorname{det}\left(g_{\mu \nu}^{\mathrm{QK}}\right)= & -\sin ^{2} \theta\left[\Sigma^{2}-\frac{5 \epsilon_{\mathrm{QK}} r^{3}}{16 M^{2}}(1+3 \cos 2 \theta)\right. \\
& {\left[2 M\left(2 M^{2}-3 M r-3 r^{2}\right)\right.} \\
& \left.\left.+3 r\left(r^{2}-2 M^{2}\right) \ln \left(\frac{r}{r-2 M}\right)\right]\right] .
\end{aligned}
$$

This determinant changes sign and hence the QK metric becomes non-Lorentzian and contains regions with closed timelike curves outside of the coordinate singularity at $r=2 M$ and just inside or outside of the event horizon if $\epsilon_{\mathrm{QK}} \neq 0$. Regions of Lorentz violations and closed timelike curves are present around the poles if 
$\epsilon_{\mathrm{QK}}>0$ and near the equatorial plane if $\epsilon_{\mathrm{QK}}<0$. In Figure 3.3, I plot these regions for the case $a=0.3 M$ and $\epsilon_{\mathrm{QK}}= \pm 0.1$.
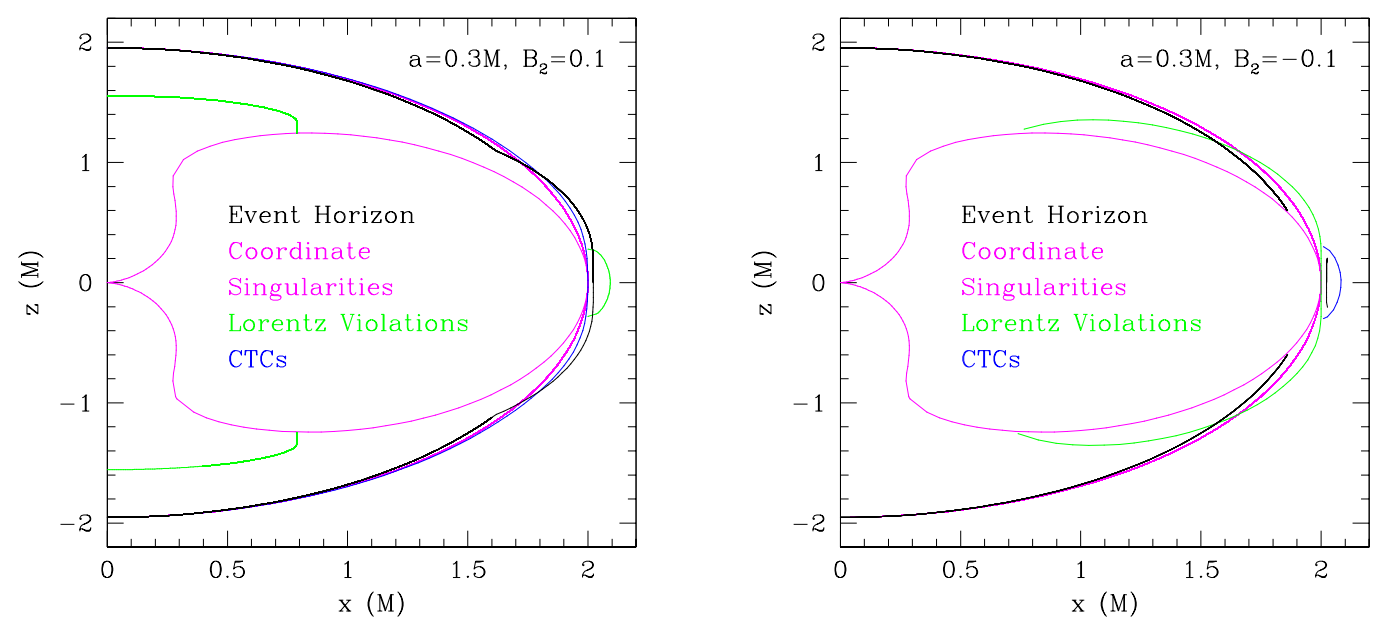

Figure 3.4 Event horizon and regions with Lorentz violations and closed timelike curves in the BK metric for $a=0.3 M$ and $B_{2}= \pm 0.1$. The event horizon is closed if $B_{2}=0.1$ and open if $B_{2}=-0.1$. In both cases, Lorentz violations and closed timelike curves occur outside of the event horizon and the outermost coordinate singularity.

In the case of the BK metric, the determinant is given by

$$
\operatorname{det}\left(g_{\mu \nu}^{\text {вк }}\right)=-\Sigma^{2} \sin ^{2} \theta\left[1-4\left(\psi_{1}-\gamma_{1}\right)\right],
$$

which becomes non-Lorentzian when

$$
\psi_{1}-\gamma_{1}>\frac{1}{4}
$$

For this spacetime, Lorentz violations and closed timelike curves occur outside of the event horizon and the outermost coordinate singularity $r_{1,+}$ around the equatorial plane if $B_{2} \neq 0$. Additional regions of Lorentz violation lie inside the event horizon. In Figure 3.4, I plot these regions for $a=0.3 M$ and $B_{2}= \pm 0.1$. 

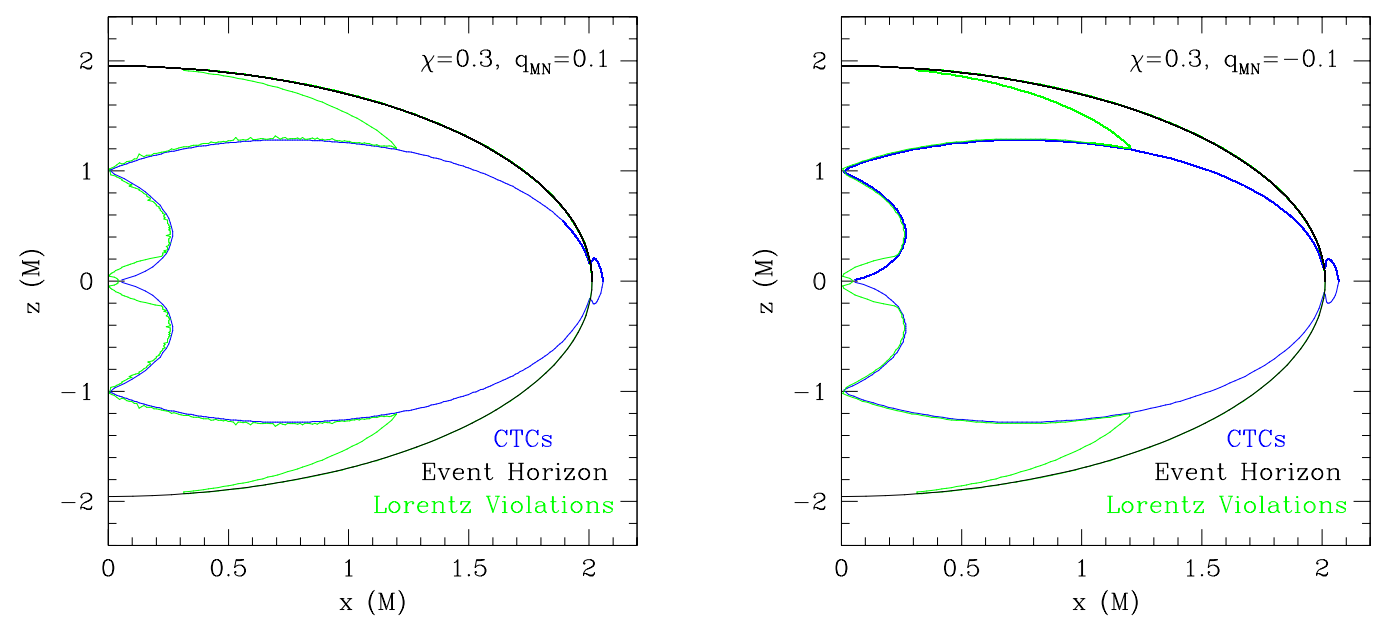

Figure 3.5 Event horizon and regions with Lorentz violations and closed timelike curves in the $\mathrm{MN}$ metric for $\chi=0.3$ and $q_{\mathrm{MN}}= \pm 0.1$. Lorentz violations lie inside and closed timelike curves occur both inside and outside of the event horizon.

The MN metric is of a much more complex form, and I will not write its determinant here explicitly. Gair et al. (2008) and Brink (2008) analyzed this metric in cylindrical coordinates without the rescaling of Section 3.1. They showed the existence of regions with closed timelike curves around the singularity. In my form of the metric, if $q_{\mathrm{MN}} \neq 0$, regions of Lorentz violation lie inside the event horizon, while closed timelike curves occur both inside and outside of the event horizon. In Fig. 3.5 I plot these regions for $|\chi|=0.3$ and $q_{\mathrm{MN}}= \pm 0.1$.

In the case of the MGBK metric, the determinant is, again, a very long expression, and I will not write it explicitly. In this chapter, I will allow only the coefficients $\gamma_{1,2}, \gamma_{3,1}, \gamma_{3,3}$, and $\gamma_{4,2}$ to be nonzero, since these are the lowest-order perturbations.

The metric is regular everywhere outside of the event horizon, and regions 


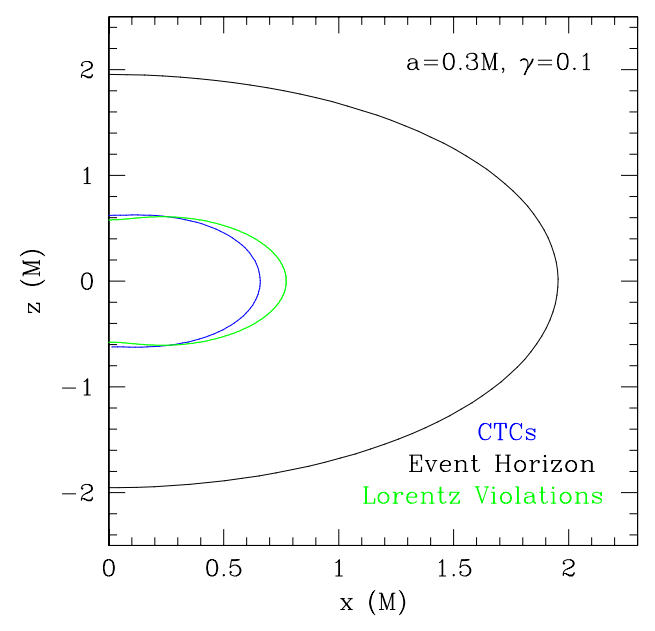

Figure 3.6 Event horizon and regions with Lorentz violations and closed timelike curves in the MGBK metric for $a=0.3 M$ and $\gamma_{1,2}=\gamma_{3,1}=\gamma_{3,3}=\gamma_{4,2}=0.1$. The metric is regular outside of the event horizon, and regions with Lorentz violations and closed timelike curves occur only inside the event horizon.

with Lorentz violations and closed timelike curves occur only inside the event horizon as long as $|\gamma| \leq 0.1$. In Figure 3.6, I plot these regions for $a=0.3 M$ and $\gamma_{1,2}=\gamma_{3,1}=\gamma_{3,3}=\gamma_{4,2}=0.1$. Violations can occur outside of the horizon when $|\gamma|>0.1$.

In my calculations, I used the far-field expansion for $h_{t \phi}$ given in Eq. (3.21) truncated at $n=3$. This means I should take the shapes of the curves of Lorentz violation and of the closed timelike curves in Fig. 3.6 with caution since they lie in the region where the far-field expansion is not valid. However, since they lie behind the event horizon, I are not overly concerned with the exact location of the regions of Lorentz violation or closed timelike curves.

In Table 3.1, I summarize the properties and pathologies of the various met- 
rics.

Pathologies of Kerr-like Black Hole Metrics

\begin{tabular}{lccccccc}
\hline \hline Metric & Solution & Ricci & Petrov & Central & Coordinate & Lorentz & CTCs \\
& Type & Flat & Type & Object & Singularities & Violations & \\
\hline QK & perturbation & yes & I & S & yes & yes & yes \\
BK & perturbation & yes $^{\mathrm{a}}$ & $\mathrm{I}$ & $\mathrm{BH} / \mathrm{S}$ & yes & yes & yes \\
MN & full solution & yes & I & BH & no & no & yes \\
JP & full solution & no & I & BH & no & no & no \\
MGBK & perturbation & no & D & BH & no & no & no \\
\hline
\end{tabular}

Table 3.1 Pathologies of Kerr-like Black Hole Metrics. The central object is either a black hole $(\mathrm{BH})$ or a naked singularity (S). Coordinate singularities, Lorentz violations, and closed timelike curves (CTCs) refer to pathologies outside of the event horizon. ${ }^{a}$ For $a=0$ only. ${ }^{b}$ See chapter 4 . 


\subsection{Conclusions}

In this chapter, I compiled several parametric frameworks of metrics that deviate from the Kerr solution. Due to the special nature of the Kerr metric in general relativity as a consequence of the no-hair theorem, all such parametric spacetimes violate at least one of the assumptions of the no-hair theorem. I analyzed in detail how each metric violates the no-hair theorem.

The QK and MN metrics as well as the BK metric in the limit $a \rightarrow 0$ are vacuum solutions in general relativity, while the MGBK metrics is not. The MGBK metric admits four conserved quantities and is of Petrov type D, while the other four metrics generally admit only three conserved quantities and are of Petrov type I. All five metrics are asymptotically flat, which I showed explicitly for the QK and MN metrics. The MN metric, however, requires an appropriate coordinate transformation and rescaling of the mass.

I showed that the QK metric harbors a naked singularity and that the $\mathrm{MN}$ and MGBK metrics harbor a black hole, while the BK metric describes either a black hole or a naked singularity.

I identified the location of coordinate singularities in the BK, MN, and MGBK metrics (see Chapter 2 for the QK metric). These can coincide with the event horizon, or they can occur either inside or outside of the event horizon. I showed that in the $\mathrm{QK}, \mathrm{BK}$, and $\mathrm{MN}$ metrics regions with Lorentz violations or closed timelike curves occur outside of the event horizon.

All of these metrics can be used for astrophysical tests of the no-hair theorem in either the electromagnetic or gravitational-wave spectrum. However, due the the existence of pathologies outside of the event horizon, the QK, BK, and MN metrics require imposing an artificial cutoff. Such cutoff radius needs to be lo- 
cated near but outside of the event horizon and to shield all pathologies from a distant observer. For tests of the no-hair theorem with gravitational waves, this cutoff plays no role, because strong-field effects manifest at radial distances larger than the cutoff for all values of the spin. For electromagnetic tests, however, strong-field effects manifest at radii that are comparable to the ISCO. Consequently, the $\mathrm{QK}, \mathrm{BK}$, and $\mathrm{MN}$ metrics can only be used for moderately spinning black holes as long as the ISCO lies outside of the cutoff radius (see discussion in Chapters 2 and 4 ).

The MGBK metric is regular everywhere outside of the event horizon as long as it remains a small perturbation of the Kerr metric. In this regime, this metric can likewise be used for electromagnetic tests of the no-hair theorem. However, since this metric is of Petrov type D, it is an ideal framework for gravitationalwave tests of the no-hair theorem (Vigeland et al. 2011; Gair \& Yunes 2011). 


\section{CHAPTER 4}

\section{A Metric For RAPIDly SPINNING BlaCK HOLES}

In this chapter, I construct such a Kerr-like black hole metric which suffers from no pathologies up to the maximum value of the spin and which contains a set of parameters that measure potential deviations from the Kerr metric in the strongfield regime. In order to achieve this in a regular manner, I start by introducing a parametric deviation to the Schwarzschild metric, following Yunes \& Stein (2011). I then apply the Newman-Janis algorithm (Newman \& Yanis 1965), as in Vigeland \& Hughes (2010), in order to generate a metric for a spinning black hole. The contents of this chapter were published in Johannsen \& Psaltis (2011c). 


\subsection{Construction of a Kerr-like Black Hole Metric}

In this section, I construct a new class of Kerr-like black hole metrics which describe a stationary, axisymmetric, and asymptotically flat vacuum spacetime. In addition to the mass and spin of the black hole, this spacetime depends on a set of parameters that measure potential deviations from the Kerr metric. My spacetime reduces smoothly to the Kerr metric if the deviations are dialed to zero.

My starting point is a Schwarzschild-like metric with the line element (Yunes \& Stein 2011)

$$
d s^{2}=-f[1+h(r)] d t^{2}+f^{-1}[1+h(r)] d r^{2}+r^{2}\left(d \theta^{2}+\sin ^{2} \theta d \phi^{2}\right)
$$

in Schwarzschild coordinates $(t, r, \theta, \phi)$, where $M$ is the mass of the central object and

$$
f \equiv 1-\frac{2 M}{r} .
$$

A metric of this form is both stationary and spherically symmetric and reduces to the Schwarzschild metric in the case $h(r)=0$. As in Yunes \& Stein (2011), I do not modify the angular part of the metric for simplicity and in order to retain spherical symmetry. Unlike Glampedakis \& Babak (2006) and Vigeland \& Hughes (2010), since I interested in constructing a black hole spacetime that is free of pathologies outside of the event horizon, I do not require my metric to be a vacuum solution of the Einstein equations. Similarly, I do not require full integrability of geodesic motion in my metric (unlike Vigeland 2011). While this property is critical for the design of waveforms for observations in the gravitationalwave spectrum, it may only simplify ray-tracing calculations for applications in the electromagnetic spectrum, but it is not a necessity. 
I choose the function $h(r)$ to be of the form

$$
h(r) \equiv \sum_{k=0}^{\infty} \epsilon_{k}\left(\frac{M}{r}\right)^{k} .
$$

The Kerr metric can be obtained from the Schwarzschild metric by the NewmanJanis algorithm (Newman \& Janis 1965), which is based on a complex coordinate transformation. Through this procedure the effect of rotation can be incorporated into a static spacetime in a natural way. In the following, I apply the NewmanJanis algorithm to the Schwarzschild-like metric given by Eq. (4.1) in order to construct a Kerr-like metric that depends on the mass $M$, spin $a$, and the set of parameters $\epsilon_{k}$.

First I perform a transformation to Eddington-Finkelstein coordinates choosing a set of new coordinates $\left(u^{\prime}, r^{\prime}, \theta^{\prime}, \phi^{\prime}\right)$, where

$$
\begin{aligned}
& u^{\prime}=t-r-2 M \ln \left(\frac{r-2 M}{2 M}\right), \\
& r^{\prime}=r, \quad \theta^{\prime}=\theta, \quad \phi^{\prime}=\phi,
\end{aligned}
$$

which yields a metric $\tilde{g}_{\mu \nu}$ of the form

$$
d s^{2}=-f[1+h(r)] d u^{2}-2[1+h(r)] d u d r+r^{2}\left(d \theta^{2}+\sin ^{2} \theta d \phi^{2}\right) .
$$

In expression (4.6) I have dropped the primes for brevity.

I express the metric $\tilde{g}_{\mu \nu}$ given by Eq. (4.6) in contravariant form in the NewmanPenrose formalism (Newman \& Penrose 1962)

$$
\tilde{g}^{\mu \nu}=-l^{\mu} n^{\nu}-l^{\nu} n^{\mu}+m^{\mu} \bar{m}^{\nu}+m^{\nu} \bar{m}^{\mu}
$$

using a complex null tetrad

$$
Z_{s}^{\mu}=\left(l^{\mu}, n^{\mu}, m^{\mu}, \bar{m}^{\mu}\right), \quad s=1,2,3,4
$$


with legs

$$
\begin{aligned}
l^{\mu} & =\delta_{r}^{\mu}, \\
n^{\mu} & =\frac{1}{1+h(r)}\left[\delta_{u}^{\mu}-\frac{1}{2}\left(1-\frac{2 M}{r}\right) \delta_{r}^{\mu}\right], \\
m^{\mu} & =\frac{1}{\sqrt{2} r}\left(\delta_{\theta}^{\mu}+\frac{i}{\sin \theta} \delta_{\phi}^{\mu}\right), \\
\bar{m}^{\mu} & =\frac{1}{\sqrt{2} r}\left(\delta_{\theta}^{\mu}-\frac{i}{\sin \theta} \delta_{\phi}^{\mu}\right) .
\end{aligned}
$$

This tetrad is orthonormal obeying the conditions

$$
\begin{aligned}
l_{\mu} m^{\mu} & =l_{\mu} \bar{m}^{\mu}=n_{\mu} m^{\mu}=n_{\mu} \bar{m}^{\mu}=0, \\
l_{\mu} l^{\mu} & =n_{\mu} n^{\mu}=m_{\mu} m^{\mu}=\bar{m}_{\mu} \bar{m}^{\mu}=0, \\
l_{\mu} n^{\mu} & =-1, \quad m_{\mu} \bar{m}^{\mu}=1 .
\end{aligned}
$$

Now I allow for the radius $r$ to take on complex values and rewrite the legs of the null tetrad in the form

$$
\begin{aligned}
l^{\mu} & =\delta_{r}^{\mu}, \\
n^{\mu} & =\frac{1}{1+h(r, \bar{r})}\left[\delta_{u}^{\mu}-\frac{1}{2}\left(1-\frac{M}{r}-\frac{M}{\bar{r}}\right) \delta_{r}^{\mu}\right], \\
m^{\mu} & =\frac{1}{\sqrt{2} r}\left(\delta_{\theta}^{\mu}+\frac{i}{\sin \theta} \delta_{\phi}^{\mu}\right), \\
\bar{m}^{\mu} & =\frac{1}{\sqrt{2} \bar{r}}\left(\delta_{\theta}^{\mu}-\frac{i}{\sin \theta} \delta_{\phi}^{\mu}\right),
\end{aligned}
$$

where an overbar denotes complex conjugation and

$$
h(r, \bar{r}) \equiv \sum_{k=0}^{\infty}\left[\epsilon_{2 k}+\epsilon_{2 k+1} \frac{M}{2}\left(\frac{1}{r}+\frac{1}{\bar{r}}\right)\right]\left(\frac{M^{2}}{r \bar{r}}\right)^{k} .
$$

Next I perform a complex coordinate transformation defining a new set of coordinates $\left(u^{\prime}, r^{\prime}, \theta^{\prime}, \phi^{\prime}\right)$ by the relations

$$
u^{\prime}=u-i a \cos \theta
$$




$$
\begin{aligned}
& r^{\prime}=r+i a \cos \theta, \\
& \theta^{\prime}=\theta, \quad \phi^{\prime}=\phi .
\end{aligned}
$$

I transform the tetrad in the usual way,

$$
Z_{s}^{\prime \mu}=\frac{\partial x^{\prime \mu}}{\partial x^{\nu}} Z_{s}^{\nu}
$$

and obtain

$$
\begin{aligned}
l^{\mu} & =\delta_{r}^{\mu} \\
n^{\mu} & =\frac{1}{1+h(r, \bar{r})}\left[\delta_{u}^{\mu}-\frac{1}{2}\left(1-\frac{2 M r}{\Sigma}\right) \delta_{r}^{\mu}\right], \\
m^{\mu} & =\frac{1}{\sqrt{2} r}\left[i a \sin \theta\left(\delta_{u}^{\mu}-\delta_{r}^{\mu}\right)+\delta_{\theta}^{\mu}+\frac{i}{\sin \theta} \delta_{\phi}^{\mu}\right], \\
\bar{m}^{\mu} & =\frac{1}{\sqrt{2} \bar{r}}\left[-i a \sin \theta\left(\delta_{u}^{\mu}-\delta_{r}^{\mu}\right)+\delta_{\theta}^{\mu}-\frac{i}{\sin \theta} \delta_{\phi}^{\mu}\right],
\end{aligned}
$$

where

$$
\begin{aligned}
\Sigma & \equiv r^{2}+a^{2} \cos ^{2} \theta \\
h(r, \theta) & \equiv \sum_{k=0}^{\infty}\left(\epsilon_{2 k}+\epsilon_{2 k+1} \frac{M r}{\Sigma}\right)\left(\frac{M^{2}}{\Sigma}\right)^{k},
\end{aligned}
$$

and, again, I have dropped the primes.

From these expressions, I recover the contravariant metric with the use of Eq. (4.7) and perform a transformation to coordinates $\left(t^{\prime}, r^{\prime}, \theta^{\prime}, \phi^{\prime}\right)$ given by the implicit relations

$$
\begin{aligned}
d u & =d t^{\prime}+\frac{r^{\prime 2}+a^{2}}{\Delta^{\prime}} d r^{\prime} \\
r & =r^{\prime}, \quad \theta=\theta^{\prime} \\
d \phi & =d \phi^{\prime}-\frac{a}{\Delta^{\prime}} d r^{\prime}
\end{aligned}
$$

where

$$
\Delta \equiv r^{2}-2 M r+a^{2}
$$


In the case that the function $h(r, \theta)$ vanishes, the metric derived in this fashion is the usual Kerr metric in Boyer-Lindquist coordinates with mass $M$ and spin $a$. For nonzero values of the function $h(r, \theta)$, however, the resulting metric contains the off-diagonal element

$$
\tilde{g}_{r \phi}=\frac{a \sum \sin ^{2} \theta}{\Delta} h(r, \theta)
$$

in addition to the usual frame-dragging element $\tilde{g}_{t \phi}$.

In order to eliminate the element $\tilde{g}_{r \phi}$, I apply another transformation to new coordinates $\left(t^{\prime}, r^{\prime}, \theta^{\prime}, \phi^{\prime}\right)$ given by the implicit relations

$$
\begin{aligned}
d t & =d t^{\prime}+F\left(r^{\prime}, \theta^{\prime}\right) d r^{\prime} \\
r & =r^{\prime} \\
\theta & =\theta^{\prime} \\
d \phi & =d \phi^{\prime}+G\left(r^{\prime}, \theta^{\prime}\right) d r^{\prime}
\end{aligned}
$$

with the functions

$$
\begin{gathered}
F\left(r^{\prime}, \theta^{\prime}\right) \equiv-\frac{\tilde{g}_{r \phi}}{\tilde{g}_{t t}}\left(\frac{\tilde{g}_{t \phi}}{\tilde{g}_{t t}}-\frac{\tilde{g}_{\phi \phi}}{\tilde{g}_{t \phi}}\right)^{-1}, \\
G\left(r^{\prime}, \theta^{\prime}\right) \equiv \frac{\tilde{g}_{r \phi}}{\tilde{g}_{t \phi}}\left(\frac{\tilde{g}_{t \phi}}{\tilde{g}_{t t}}-\frac{\tilde{g}_{\phi \phi}}{\tilde{g}_{t \phi}}\right)^{-1} .
\end{gathered}
$$

Finally (dropping the primes), I arrive at the following metric $g_{\mu \nu}$ given by the line element

$$
\begin{aligned}
d s^{2}= & -[1+h(r, \theta)]\left(1-\frac{2 M r}{\Sigma}\right) d t^{2}-\frac{4 a M r \sin ^{2} \theta}{\Sigma}[1+h(r, \theta)] d t d \phi \\
& +\frac{\Sigma[1+h(r, \theta)]}{\Delta+a^{2} \sin ^{2} \theta h(r, \theta)} d r^{2}+\Sigma d \theta^{2} \\
& +\left[\sin ^{2} \theta\left(r^{2}+a^{2}+\frac{2 a^{2} M r \sin ^{2} \theta}{\Sigma}\right)\right.
\end{aligned}
$$




$$
\left.+h(r, \theta) \frac{a^{2}(\Sigma+2 M r) \sin ^{4} \theta}{\Sigma}\right] d \phi^{2},
$$

which reduces to the Kerr metric in Boyer-Lindquist coordinates in the case $h(r, \theta)=0$ and to the generalized Schwarzschild metric given by Eq. (4.1) if $a=0$.

The metric $g_{\mu \nu}$ that I have constructed in this manner is both stationary and axisymmetric. As I will argue in the following, the nontrivial dependence of my metric on the function $h(r, \theta)$ ensures the preservation of the properties of the Kerr metric that are critical for observational tests of the no-hair theorem. In general relativity, the Einstein tensor of my metric is nonzero unless $h(r, \theta)$ vanishes. Therefore, I regard my metric as a vacuum spacetime of an appropriately chosen set of field equations which are unknown but different from the Einstein equations for nonzero $h(r, \theta)$. For observational tests of the no-hair theorem in the electromagnetic spectrum, the field equations are not needed explicitly (Psaltis 2009), and I only require a spacetime and the validity of the Einstein equivalence principle (c.f., Will 1993), which governs the motion of particles in that spacetime.

I justify the nature of my metric in Section 4, where I show that its properties are very similar to the ones of the Kerr metric. In particular, I compute the location of the event horizon. The requirement of asymptotic flatness imposes restrictions on the function $h(r, \theta)$, which I will address in the next section. 
4.2 Constraints on the Function $h(r, \theta)$
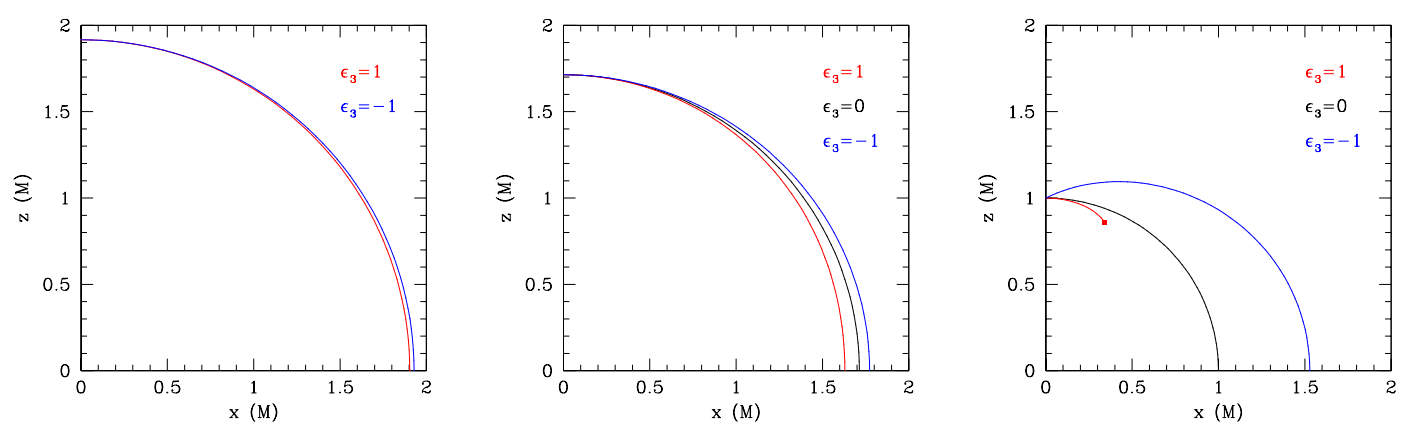

Figure 4.1 Event horizon of a non-Kerr black hole in the $x z$-plane for values of the spin (left) $a=0.4 M$, (middle) $a=0.7 M$, and (right) $a=1.0 M$ and several values of the parameter $\epsilon_{3}$. For positive values of the parameter $\epsilon_{3}$, the event horizon has a more prolate shape than the horizon of a Kerr black hole with the same spin, while for negative values of the parameter $\epsilon_{3}$, the shape is more oblate. In the case $a=M$, the event horizon has a dumbbell shape if $\epsilon_{3}=-1$ and is not closed if $\epsilon_{3}=1$.

In this section, I constrain the form of the function $h(r, \theta)$ given by Eq. (B.4) by the requirements that the metric $g_{\mu \nu}$ given by Eq. (B.1) is asymptotically flat and consistent with observational weak-field constraints on deviations from the Kerr metric. The resulting metric, then, is suitable for the exploration of the strongfield regime in the vicinity of black holes.

In Newtonian gravity and at a large distance from the source, the potential of an extended body approaches that of a spherical body of equal mass. Similarly, in general relativity, stationary and asymptotically flat spacetimes are Schwarzschild-like in the limit of large radii in an appropriately chosen coordinate system, i.e., they fall off as $1 / r$ or faster (Beig 1980; Beig \& Simon 1981). In 
that particular gauge, such metrics are of the asymptotic form (e.g., Heusler 1996)

$$
\begin{aligned}
d s^{2}= & -\left[1-\frac{2 M}{r}+\mathcal{O}\left(r^{-2}\right)\right] d t^{2}-\left[\frac{4 a}{r} \sin ^{2} \theta+\mathcal{O}\left(r^{-2}\right)\right] d t d \phi \\
+ & {\left[1+\mathcal{O}\left(r^{-1}\right)\right]\left[d r^{2}+r^{2} d \Omega^{2}\right] }
\end{aligned}
$$

where I used the notation

$$
d \Omega^{2}=d \theta^{2}+\sin ^{2} \theta d \phi^{2}
$$

Asymptotically flat spacetimes with a slower fall-off involve gravitational radiation (Kennefick \& Ó Murchadha 1995) and, thus, cannot be stationary.

A similar argument must hold for more general spacetimes that are not necessarily a solution of the Einstein equations. For $r \gg M$ and $r \gg a$, my metric given by Eq. (B.1) has the asymptotic form

$$
\begin{aligned}
d s^{2} \approx & -\left[1-\frac{2 M}{r}+h(r)\right] d t^{2}-\frac{4 a[1+h(r)]}{r} \sin ^{2} \theta d t d \phi \\
+ & {\left[1+\frac{2 M}{r}+h(r)\right] d r^{2}+r^{2} d \Omega }
\end{aligned}
$$

where $h(r)$ is given by Eq. (4.3). Therefore, the function $h(r)$ must be of order $\mathcal{O}\left(1 / r^{n}\right)$ with $n \geq 2$, and I conclude that $\epsilon_{0}=\epsilon_{1}=0$.

Limits on the parameter $\epsilon_{2}$ of the next leading-order term in the function $h(r)$ can readily be obtained from the observational constraints on weak-field deviations from general relativity in the parameterized post-Newtonian (PPN) framework (Will 2006). In the PPN approach, the asymptotic spacetime is expressed as

$$
d s^{2}=-A(r) d t^{2}+B(r) d r^{2}+r^{2} d \Omega
$$

where

$$
A(r)=1-\frac{2 M}{r}+2(\beta-\gamma) \frac{M^{2}}{r^{2}}
$$




$$
B(r)=1+2 \gamma \frac{M}{r} .
$$

In general relativity, $\beta=\gamma=1$.

From the asymptotic form of the metric given by Eq. (4.45), I identify

$$
\begin{aligned}
& \epsilon_{2}=2(\beta-1), \\
& \gamma=1 .
\end{aligned}
$$

The best current PPN contraint on the parameter $\beta$ is set by the Lunar Laser Ranging experiment and yields (Williams et al. 2004a)

$$
|\beta-1| \leq 2.3 \times 10^{-4},
$$

if the weak equivalence principle is satisfied, which I assume throughout the chapter. Therefore, this limit implies that

$$
\left|\epsilon_{2}\right| \leq 4.6 \times 10^{-4} .
$$

For the remainder of this chapter, I will set $\epsilon_{2}=0$ and explore in some detail metrics with $\epsilon_{k}=0$ for $k>3$. In this case, the function $h(r, \theta)$ reduces to

$$
h(r, \theta)=\epsilon_{3} \frac{M^{3} r}{\Sigma^{2}} .
$$

The parameter $\epsilon_{3}$ is unconstrained by current observational tests of general relativity (c.f., Psaltis 2008a). My metric with this choice of $h(r, \theta)$, therefore, allows me to probe the regime of strong-field gravity in parametric form. 


\subsection{Metric Properties}

In this section, I analyze some of the properties of the metric given by Eq. (B.1), and I choose the function $h(r, \theta)$ according to Eq. (B.5) for simplicity. In particular, I determine the range of the parameters $a$ and $\epsilon_{3}$ for which my metric describes a black hole. A similar analysis should be valid for all higher orders in $M / r$. Unless the parameter $\left|\epsilon_{3}\right|$ is very small, I expect potential strong-field deviations from the Kerr metric to be most easily detectable at order $(M / r)^{3}$.

\subsubsection{Event Horizon}

First, I calculate the location of the event horizon, which occurs at the root of the equation

$$
g_{t \phi}^{2}-g_{t t} g_{\phi \phi}=0 .
$$

This equation can be rewritten in the form

$$
\left(1+\epsilon_{3} \frac{M^{3} r}{\Sigma^{2}}\right) w\left(r, \theta ; M, a, \epsilon_{3}\right)=0,
$$

where $w\left(r, \theta ; M, a, \epsilon_{3}\right)$ is a function of the radius $r$ and the angle $\theta$, as well as of the mass $M$, spin $a$, and the parameter $\epsilon_{3}$. This equation can have more than one root leading to the presence of both an inner and an outer horizon similar to the case of the Kerr metric. Since in this chapter I are only concerned with the exterior spacetime, I will refer hereafter to the outer horizon simply as the event horizon. As shown in Bambi \& Modesto (2011), this condition is equivalent to the more general condition (3.30) for values of the spin $|a| \leq M$.

In Figure 4.1, I plot the event horizon in the $x z$-plane for several values of the spin $a$ and the parameter $\epsilon_{3}$. The horizon is more prolate than the horizon of a Kerr black hole with the same spin for positive values of the parameter $\epsilon_{3}$, while 
it is more oblate for negative values of the parameter $\epsilon_{3}$. In the case $a=M$, the event horizon has a dumbbell shape if $\epsilon_{3}=-1$ and is not closed if $\epsilon_{3}=1$.

In this chapter, I are only interested in black holes, i.e., in compact objects for which the event horizon is entirely closed. In the case $a=0$, the event horizon is a sphere with radius $r_{\mathrm{h}}=2 M$, if $\epsilon_{3} \geq-8$, or $r_{\mathrm{h}}=\left(\left|\epsilon_{3}\right|\right)^{1 / 3} M$, if $\epsilon_{3}<-8$. For negative values of the parameter $\epsilon_{3}$, the event horizon is always closed, because the first factor in Eq. (4.55) vanishes at some $r>0$ for all $0 \leq \theta<\pi$. For positive values of the parameter $\epsilon_{3}$, the first factor in Eq. (4.55) is always positive, while the existence of a root of the function $w\left(r, \theta ; M, a, \epsilon_{3}\right)$ depends on the value of the parameter $\epsilon_{3}$. For each value of the spin $|a|>0$, there exists a value of the parameter $\epsilon_{3}>0$ such that the event horizon is no longer closed. A hole appears in the event horizon around the equatorial plane within the range $\theta=\pi / 2 \pm \theta_{\text {hole }}$, and the central object becomes a naked singularity.

In Figure 4.2, I delineate the part of the parameter space, within which the event horizon is closed, and the central object is a black hole. The solid line marks the upper limit on the parameter $\epsilon_{3}$ as a function of the spin, for which the event horizon is still closed. The shaded region corresponds to the excluded part of the parameter space, where the central object is a naked singularity. In principle, the parameter space can be expanded to include values of the parameter $\left|\epsilon_{3}\right|>10$. However, I will not consider this case here, because this relatively large range of the parameter $\epsilon_{3}$ should already suffice to study strong-field deviations from the Kerr metric.

The Kerr metric describes a black hole only for values of the spin $|a| \leq M$. In the case $|a|>M$, this spacetime contains a naked singularity, and causality is violated at every point in space due to the presence of closed timelike curves 


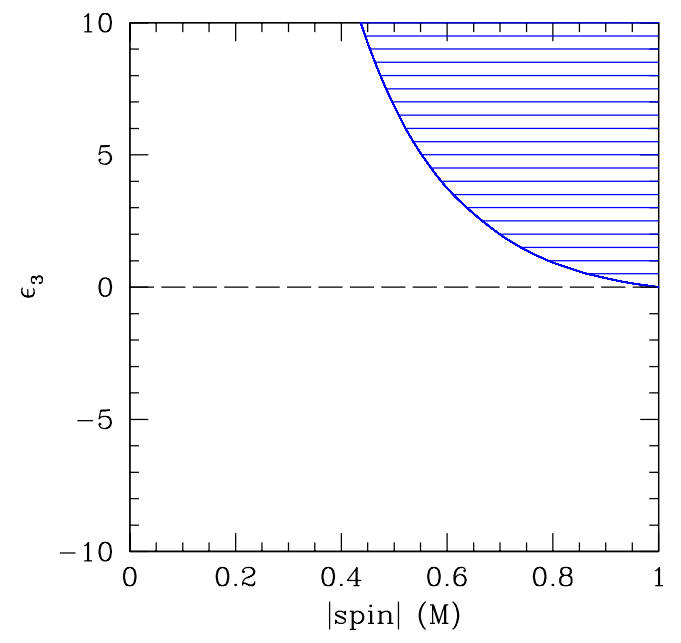

Figure 4.2 Maximum values of the parameter $\epsilon_{3}$ versus the spin $a$, for which the event horizon is entirely closed. The shaded region marks the part of the parameter space where the central object is a naked singularity. Outside of this region, the central object is a black hole, which is described by its mass $M$, spin $a$ and the parameter $\epsilon_{3}$. The dashed line corresponds to a Kerr black hole.

(Carter 1968, 1973). In my metric, the event horizon is not closed if $|a|>M$, unless $\epsilon_{3}<-16|a|^{3} / 3 \sqrt{3}$. In this case, my metric describes a superspinning black hole (c.f. Bambi \& Freese 2009). I will not consider this case either, because the Kerr metric itself is unphysical in this spin range.

Analyzing the elements of my metric, I find that $g_{\theta \theta}>0$ throughout the spacetime and $g_{r r}>0, g_{\phi \phi}>0$ outside of the event horizon. Consequently, my (exterior) spacetime is free of closed timelike curves, and causality is satisfied. 


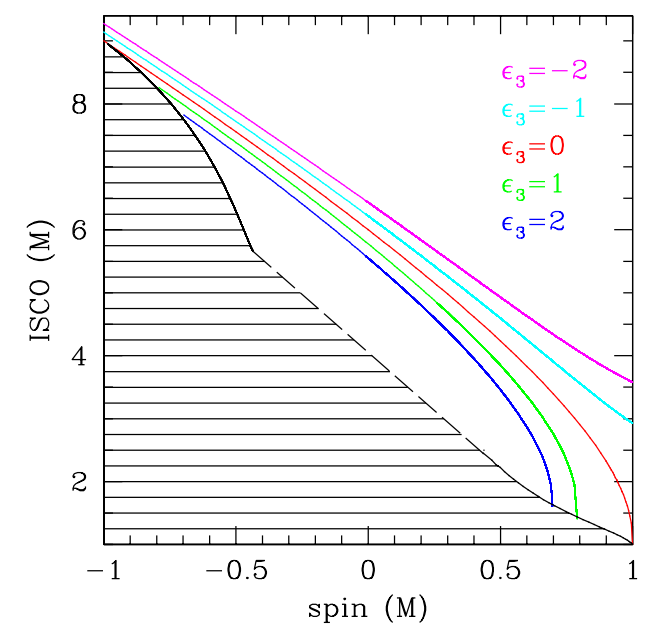

Figure 4.3 Radius of the ISCO as a function of the spin $a$ for several values of the parameter $\epsilon_{3}$. The radius of the ISCO decreases with increasing values of the parameter $\epsilon_{3}$. The shaded region marks the excluded part of the parameter space.

\subsubsection{Energy and Axial Angular Momentum for a Particle on a Circular Equato- rial Orbit}

Here I derive expressions for the energy $E$ and axial angular momentum $L_{z}$ of a particle on a circular equatorial orbit. My derivation is similar to the ones in Bardeen (1973) for the Kerr metric and in Chapter 2 for the quasi-Kerr metric.

Since my metric is stationary and axisymmetric, there exist three integrals of the motion. For a particle with 4-momentum

$$
p^{\alpha}=\mu \frac{d x^{\alpha}}{d \tau},
$$

these constants are its rest mass $\mu$, energy $E=-p_{t}$, and axial angular momentum $L_{z}=p_{\phi}$.

The Kerr metric (in Boyer-Lindquist coordinates) is of Petrov-type D, which 


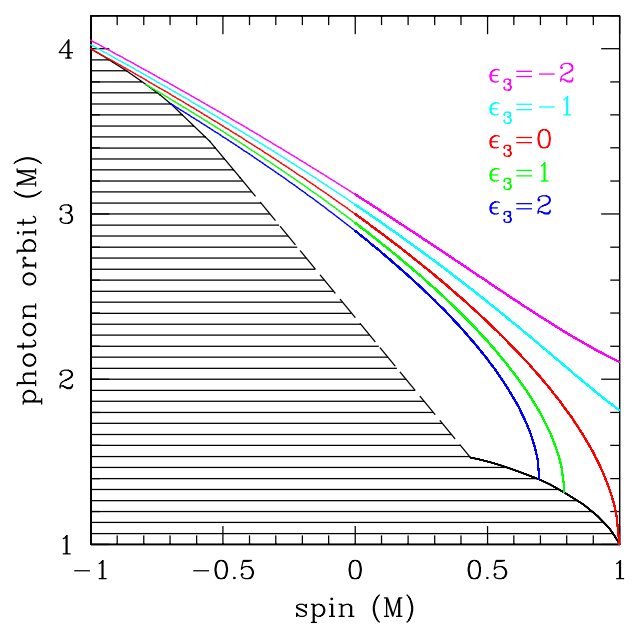

Figure 4.4 Radius of the circular photon orbit as a function of the spin $a$ for several values of the parameter $\epsilon_{3}$. The radius of the circular photon decreases with increasing values of the parameter $\epsilon_{3}$. The shaded region marks the excluded part of the parameter space.

ensures the existence of a fourth constant of the motion (Carter 1968). My metric in the form given by Eq. (B.1) with the function $h(r, \theta)$ chosen according to Eq. (B.5) is of Petrov-type I, and the fourth constant is lost. However, thanks to the reflexion symmetry of my spacetime, equatorial trajectories are fully characterized by the rest mass, energy, and axial angular momentum alone.

I solve the equation

$$
p_{\alpha} p^{\alpha}=-\mu^{2}
$$

in the equatorial plane for the radial momentum and insert the constants of the motion. I obtain

$$
\left(\frac{d r}{d \tau}\right)^{2} \equiv R(r) \equiv-\frac{1}{g_{r r}}\left(g^{t t} E^{2}-2 g^{t \phi} E L_{z}+g^{\phi \phi} L_{z}^{2}+\mu^{2}\right),
$$

where $g_{\alpha \beta}$ is my metric given by Eqs. (B.1) and (B.5). 


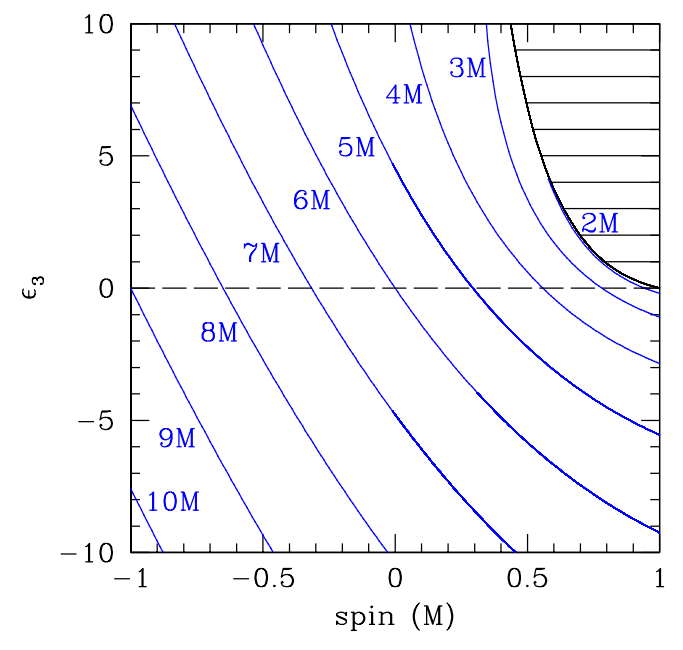

Figure 4.5 Contours of constant radius of the ISCO for values of the spin $-1 \leq$ $a / M \leq 1$ and of the parameter $-10 \leq \epsilon_{3} \leq 10$. The radius of the ISCO decreases for increasing values of the spin and the parameter $\epsilon_{3}$. The shaded region marks the excluded part of the parameter space. The dashed line corresponds to the parameter space for a Kerr black hole.

I solve the system of equations

$$
\begin{aligned}
R(r) & =0, \\
\frac{d}{d r} R(r) & =0
\end{aligned}
$$

for the energy and axial angular momentum and find the expressions

$$
\begin{aligned}
\frac{E}{\mu}= & \frac{1}{r^{6}} \sqrt{\frac{P_{1}+P_{2}}{P_{3}}} \\
\frac{L_{z}}{\mu}= & \pm \frac{1}{r^{4} P_{6} \sqrt{P_{3}}}\left[\sqrt{M\left(r^{3}+\epsilon_{3} M^{3}\right) P_{5}}\right. \\
& \left.\mp 6 a M\left(r^{3}+\epsilon_{3} M^{3}\right) \sqrt{P_{1}+P_{2}}\right] .
\end{aligned}
$$

In these expressions, the upper signs refer to a particle that corotates with the black hole, while the lower signs refer to a counterrotating particle. The functions 


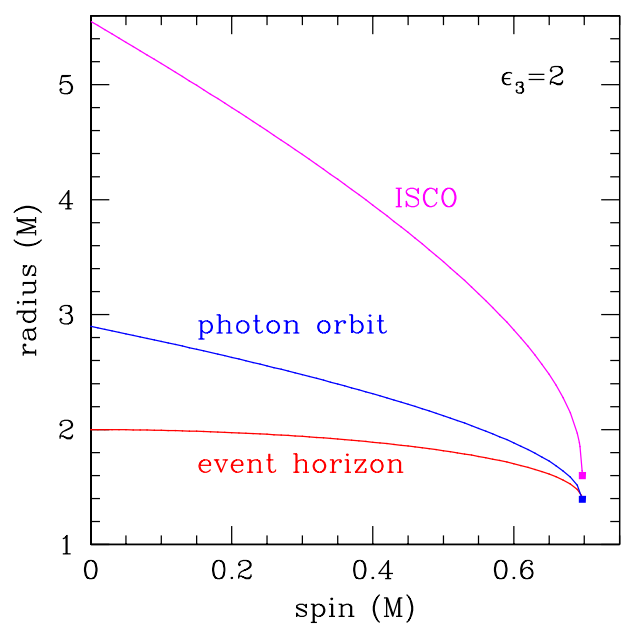

Figure 4.6 Equatorial radius of the event horizon, the circular photon orbit, and the ISCO as a function of the spin $a$ for a value of the parameter $\epsilon_{3}=2$. The event horizon and the circular photon orbit coincide at $r \approx 1.39 M$ at a spin of $a \approx 0.697 M$, while the ISCO reaches a value of $r \approx 1.60 M$.

$P_{1}$ to $P_{6}$ can be found in Appendix B.

In the Kerr limit, $\epsilon_{3} \rightarrow 0$, these expressions simplify to the corresponding ones for the Kerr metric (Bardeen 1973)

$$
\frac{E}{\mu}=\frac{r^{3 / 2}-2 M r^{1 / 2} \pm a M^{1 / 2}}{r^{3 / 4} \sqrt{r^{3 / 2}-3 M r^{1 / 2} \pm 2 a M^{1 / 2}}}
$$

and

$$
\frac{L_{z}}{\mu}= \pm \frac{M^{1 / 2}\left(r^{2} \mp 2 a M^{1 / 2} r^{1 / 2}+a^{2}\right)}{r^{3 / 4} \sqrt{r^{3 / 2}-3 M r^{1 / 2} \pm 2 a M^{1 / 2}}} .
$$

\subsubsection{Innermost Stable Circular Orbit and Circular Photon Orbit}

From the expressions (4.61) and (4.62) for the energy and axial angular momentum, I derive the locations for the ISCO and the circular photon orbit. In order to 


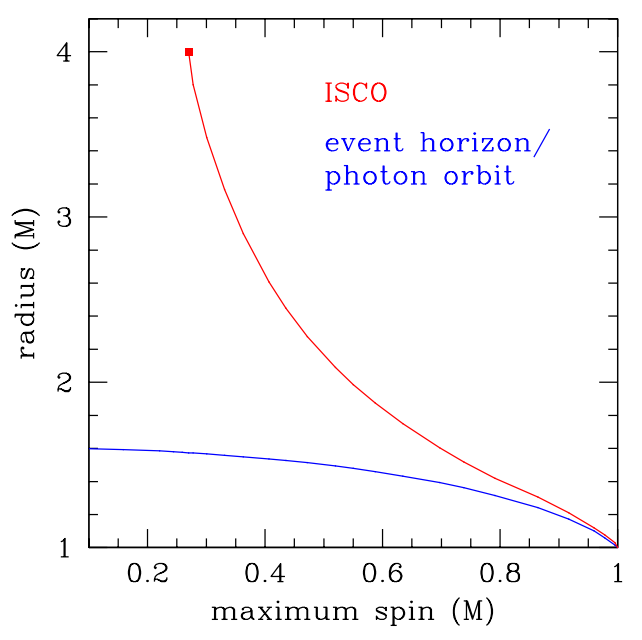

Figure 4.7 Radii of the (equatorial) event horizon and the prograde circular photon orbit and ISCO as a function maximum spin. The event horizon and the circular photon orbit coincide for all values of the maximum spin. The prograde ISCO at these values of the spin is located at slightly larger radii and merges with the event horizon and the circular photon orbit in the Kerr limit $a_{\max }=M$. For values of the maximum spin smaller than (red dot) $a_{\max } \approx 0.270 M$, multiple ISCOs occur (not shown).

obtain the ISCO, I numerically solve the equation

$$
\frac{d E}{d r}=0
$$

The photon orbit occurs at the radius at which

$$
\frac{E}{\mu} \rightarrow \infty, \quad \frac{L_{z}}{\mu} \rightarrow \infty,
$$

and the denominators in the expressions (4.61) and (4.62) vanish. Compared to the denominator of the energy $E / \mu$, the denominator of the angular momentum $L_{z}$ contains the additional factor $P_{6}$. This factor, however, has no real roots, and I can determine the radius of the photon orbit uniquely. Similar calculations have also been performed by Shibata \& Sasaki (1998) and Berti \& Stergioulas (2004). 
In Figures 4.3 and 4.4, I plot, respectively, the ISCO and the circular photon orbit as a function of the spin for several values of the parameter $\epsilon_{3}$. The radii of the ISCO and of the circular photon orbit decrease with increasing values of the parameter $\epsilon_{3}$. The shaded regions mark the excluded part of the parameter space in accordance with Figure 4.2. A spacetime with an ISCO or photon orbit radius inside the shaded regions would have an open event horizon. The solid lines along the boundary of the excluded part correspond to the locations of the ISCO and the circular photon orbit, respectively, for the range of the parameter $0 \leq \epsilon_{3} \leq 10$. I do not calculate the boundary for values of the parameter $\left|\epsilon_{3}\right|>10$ explicitly and estimate its location in both figures by a dashed line.

In Figure 4.5, I plot contours of constant radius of the ISCO as a function of spin and the parameter $\epsilon_{3}$. The radius of the ISCO decreases for increasing values of the spin and the parameter $\epsilon_{3}$. The shaded region marks the excluded part of the parameter space. The dashed line corresponds to the parameter space for a Kerr black hole, which depends only on the spin.

In the Kerr metric in Boyer-Lindquist coordinates, the equatorial event horizon, the prograde circular photon orbit, and the ISCO coincide at maximum spin $a=M$ even though their proper separation is distinct. For values of the spin exceeding this upper bound, the central object is no longer a black hole. In my metric, the upper bound depends on both the spin and the parameter $\epsilon_{3}$ as I have already shown in Figure 4.2. Along this curve, the equatorial event horizon and the prograde circular photon orbit merge within numerical accuracy, while the prograde ISCO is located at a radius slightly outside of the event horizon.

I illustrate this behavior in Figure 4.6, where I plot the equatorial radius of the event horizon, the circular photon orbit, and the ISCO as a function of the spin $a$ 
for a value of the parameter $\epsilon_{3}=2$. At a spin of $a \approx 0.697 M$, the circular photon orbit merges with the event horizon at $r \approx 1.39 M$, while the ISCO reaches a value of $r \approx 1.60 M$. For values of the spin larger than the upper bound $a \approx 0.697 M$, the event horizon is no longer closed.

In Figure 4.7, I plot the radii of the equatorial event horizon and of the prograde circular photon orbit and ISCO as a function of the maximum spin. The event horizon and the circular photon orbit coincide for all values of the maximum spin reaching the asymptotic value $r=2.0 \mathrm{M}$ in the limit $a_{\max } \rightarrow 0, \epsilon_{3} \rightarrow \infty$. The prograde ISCO at these values of the spin is located at slightly larger radii and merges with the event horizon and the circular photon orbit in the Kerr limit $a_{\max }=M$. For values of the maximum spin smaller than $a_{\max } \approx 0.270 M$ (corresponding to a value of the parameter $\epsilon_{3} \approx 32$ ), the prograde ISCO is no longer unique, and a second region of stable circular orbits occurs between the origin and the ISCO separated by a gap. This region is bound by both another innermost as well as an outermost stable orbit ("OSCO"). This region lies outside of the parameter space that I consider in this chapter (c.f., Figure 4.2). A similar effect in other parametric spacetimes has also been reported in Gair et al. (2008). 


\subsection{Conclusions}

Thanks to the no-hair theorem, any parametric deviation from the Kerr metric in general relativity does not harbor a black hole and is often plagued with unphysical properties that have to be excluded by imposing a cutoff near but outside of the event horizon. Within general relativity, tests of the no-hair theorem that are based on observational signals originating from the vicinity of the circular photon orbit or the ISCO are, therefore, limited to the region outside of the cutoff, and, so far, it has been unknown how to include rapidly spinning black holes in such tests.

In this chapter, I constructed a Kerr-like metric of a rapidly spinning black hole, which depends on a set of free parameters in addition to the mass and spin and which reduces smoothly to the Kerr metric if all parameters vanish. I showed that this metric is stationary, axisymmetric, and asymptotically flat and argued that it describes a vacuum spacetime for a set of appropriately chosen field equations. I used the current results from Lunar Laser Ranging tests of weak-field general relativity to constrain the set of free parameters.

For the case of one additional parameter, I showed that my metric is regular and free of unphysical properties outside of the event horizon and that it can be used to describe black holes up to the maximum value of the spin $a$. For positive values of the free parameter, this upper bound is a function of the deviation and smaller than the Kerr value $a_{\max }=M$. Otherwise, the upper bound coincides with the Kerr limit. For values of the spin $|a|>M$ and of the parameter $\epsilon_{3}<$ $-16|a|^{3} / 3 \sqrt{3}$, my metric describes a superspinning black hole.

We calculated expressions for the energy and angular momentum of a particle on a circular equatorial orbit and used them to obtain the locations of the ISCO 
and the circular photon orbit, respectively. Both radii decrease with increasing values of the spin and the deviation parameter. At the maximum value of the spin for a given value of the deviation, the circular photon orbit merges with the event horizon within numerical accuracy as in the Kerr metric, and the ISCO is located slightly outside of the horizon.

My metric is, thus, fully applicable in the strong-field regime arbitrarily close to the event horizon of a black hole and an ideal spacetime for astrophysical tests of the no-hair theorem that probe the immediate vicinity of black holes and that do not rely on the field equations explicitly. 


\section{CHAPTER 5}

\section{RAY-TRACING ALGORITHM}

In this chapter, I present a new ray-tracing algorithm for calculating the observational appearance of spinning compact objects with arbitrary quadrupole moments. I employ the metric of Glampedakis \& Babak (2006), which is characterized by three parameters: the mass and spin of the compact object and its quadrupole moment. In the algorithm, I integrate two first-order differential equations that arise from integrals of motion as well as two second-order differential equations for two components of the geodesic equations in order to compute the trajectories of photons in these spacetimes. I also use a third integral of motion related to the norm of the photon 4-momenta in order to monitor the accuracy of the calculations. The contents of this chapter were published in Psaltis \& Johannsen (2012). 


\subsection{A Vacuum Metric with an Arbitrary Quadrupole Moment}

I describe the external spacetime of a spinning compact object with an arbitrary quadrupole moment using the metric of Glampedakis \& Babak (2006). This metric arises by adding to the Kerr solution a contribution that has an arbitrary quadrupole moment and is by itself a solution to the vacuum Einstein field equations (Hartle \& Thorne 1968).

The metric is specified uniquely by three parameters: the mass $M$ of the compact object, the spin $a$, and the deviation $\epsilon$ of its quadrupole moment from the Kerr value. Setting $\epsilon=0$ makes the metric equal to the Kerr solution, which is appropriate for a black hole of arbitrary spin. On the other hand, when $a / M \lesssim 0.4$, all moments of the spacetime that are higher than the quadrupole are negligible and the metric becomes appropriate for neutron stars that are spinning moderately but not close to their mass shedding limit (Hartle \& Thorne 1968).

Calculating the observational appearance of a black hole that violates the nohair theorem using the quasi-Kerr metric requires a phenomenological scheme to handle its irregularities at $r \simeq 2 M$ (see Glampedakis \& Babak 2006; Gair et al. 2008; Johannsen \& Psaltis 2010a). Precisely because of the no hair theorem, the only axisymmetric vacuum solution to the Einstein field equations that does not contain naked singularities or closed time-like loops is the Kerr metric. Allowing for deviations of the quadrupole moment while requiring that the spacetime remains a solution to the field equations is necessarily accompanied by the introduction of pathologies to the spacetime. In the case of the metric (2.13) and for spins $a / M \lesssim 0.4$, these pathologies appear at $r \leq 2.6 M$ (Johannsen \& Psaltis 2010a). I consider these to be unphysical and handle them by removing from the domain of solution all inbound photons that cross $r=2.6 \mathrm{M}$. Because, for 
$a / M \lesssim 0.4$, this radius is smaller than the radius of the photon orbit, I expect that this scheme affects only marginally the images and spectra seen by an observer at infinity. 


\subsection{The Ray Tracing Algorithm}

In this section, I describe the numerical algorithm for the calculation of the trajectories of individual photons from the image plane of an observer at infinity to the location of their emission. Depending on the problem at hand, the latter may be the stellar surface or an accretion disk. Following Cadeau et al. (2007), I use two integrals of motion to write first-order differential equations for the time coordinate and the azimuth of each photon trajectory. I then complete the system using the second-order differential equations for the geodesics along the radial and polar coordinates.

The metric (2.13) is stationary and axisymmetric. It is, therefore, characterized by the two usual Killing vectors, $\xi=(1,0,0,0)$ and $\eta=(0,0,0,1)$, which correspond to the conservation of energy

$$
E=-g_{t t} \frac{d t}{d \lambda}-g_{t \phi} \frac{d \phi}{d \lambda}
$$

and angular momentum

$$
L=g_{\phi \phi} \frac{d \phi}{d \lambda}+g_{t \phi} \frac{d t}{d \lambda}
$$

along the photon trajectory. Here, $g_{\mu \nu}$ is the $\mu \nu$-element of the metric, and $\lambda$ is an affine parameter. Using these two conserved quantities, I now write two firstorder differential equations for the evolution of the $t$ - and $\phi-$ components of the photon position as

$$
\frac{d t}{d \lambda^{\prime}}=\frac{-g_{\phi \phi}-b g_{t \phi}}{g_{\phi \phi} g_{t t}-g_{t \phi}^{2}}
$$

and

$$
\frac{d \phi}{d \lambda^{\prime}}=\frac{b g_{t t}+b g_{t \phi}}{g_{\phi \phi} g_{t t}-g_{t \phi}^{2}} .
$$

where I have defined the normalized affine parameter $\lambda^{\prime} \equiv E \lambda$ and the impact parameter for the photon trajectory $b \equiv L / E$. 
For the $r$ - and $\theta$-components of the photon position I use the second-order geodesic equations, which for a general axisymmetric metric take the form

$$
\begin{aligned}
\frac{d^{2} r}{d \lambda^{\prime 2}}= & -\Gamma_{t t}^{r}\left(\frac{d t}{d \lambda^{\prime}}\right)^{2}-\Gamma_{r r}^{r}\left(\frac{d r}{d \lambda^{\prime}}\right)^{2}-\Gamma_{\theta \theta}^{r}\left(\frac{d \theta}{d \lambda^{\prime}}\right)^{2}-\Gamma_{\phi \phi}^{r}\left(\frac{d \phi}{d \lambda^{\prime}}\right)^{2} \\
& -2 \Gamma_{\phi t}^{r}\left(\frac{d \phi}{d \lambda^{\prime}}\right)\left(\frac{d t}{d \lambda^{\prime}}\right)-2 \Gamma_{\theta r}^{r}\left(\frac{d \theta}{d \lambda^{\prime}}\right)\left(\frac{d r}{d \lambda^{\prime}}\right)
\end{aligned}
$$

and

$$
\begin{aligned}
\frac{d^{2} \theta}{d \lambda^{\prime 2}}= & -\Gamma_{t t}^{\theta}\left(\frac{d t}{d \lambda^{\prime}}\right)^{2}-\Gamma_{r r}^{\theta}\left(\frac{d r}{d \lambda^{\prime}}\right)^{2}-\Gamma_{\theta \theta}^{\theta}\left(\frac{d \theta}{d \lambda^{\prime}}\right)^{2}-\Gamma_{\phi \phi}^{\theta}\left(\frac{d \phi}{d \lambda^{\prime}}\right)^{2} \\
& -2 \Gamma_{\phi t}^{\theta}\left(\frac{d \phi}{d \lambda^{\prime}}\right)\left(\frac{d t}{d \lambda^{\prime}}\right)-2 \Gamma_{\theta r}^{\theta}\left(\frac{d \theta}{d \lambda^{\prime}}\right)\left(\frac{d r}{d \lambda^{\prime}}\right)
\end{aligned}
$$

Here, $\Gamma_{\beta \gamma}^{\alpha}$ are the various Christoffel symbols for the metric (2.13).

A final integral of motion arises from the requirement that the norm of the photon 4-momentum has to vanish, i.e.,

$$
g_{t t}\left(\frac{d t}{d \lambda^{\prime}}\right)^{2}+g_{r r}\left(\frac{d r}{d \lambda^{\prime}}\right)^{2}+g_{\theta \theta}\left(\frac{d \theta}{d \lambda^{\prime}}\right)^{2}+g_{\phi \phi}\left(\frac{d \phi}{d \lambda^{\prime}}\right)^{2}+2 g_{t \phi}\left(\frac{d t}{d \lambda^{\prime}}\right)\left(\frac{d \phi}{d \lambda^{\prime}}\right)=0 .
$$

This integral of motion is not useful for replacing either the geodesic equation (5.5) or (5.6) because it contains the squares of the derivatives of the $r$ - and $\theta$ - coordinates with respect to the affine parameter. Keeping track of the appropriate sign for the two derivatives, especially near the inflection points of the geodesics, would more than offset the benefit of using a first-order integral of motion as opposed to a second-order geodesic equation. Therefore, following Cadeau et al. (2007), I use this integral of motion only in order to monitor the accuracy of the calculation. To this end, I define the parameter

$$
\begin{aligned}
\xi \equiv & {\left[g_{r r}\left(\frac{d r}{d \lambda^{\prime}}\right)^{2}+g_{\phi \phi}\left(\frac{d \phi}{d \lambda^{\prime}}\right)^{2}+g_{\theta \theta}\left(\frac{d \theta}{d \lambda^{\prime}}\right)^{2}+2 g_{t \phi}\left(\frac{d t}{d \lambda^{\prime}}\right)\left(\frac{d \phi}{d \lambda^{\prime}}\right)\right] } \\
& /\left[g_{t t}\left(\frac{d t}{d \lambda^{\prime}}\right)^{2}\right]
\end{aligned}
$$




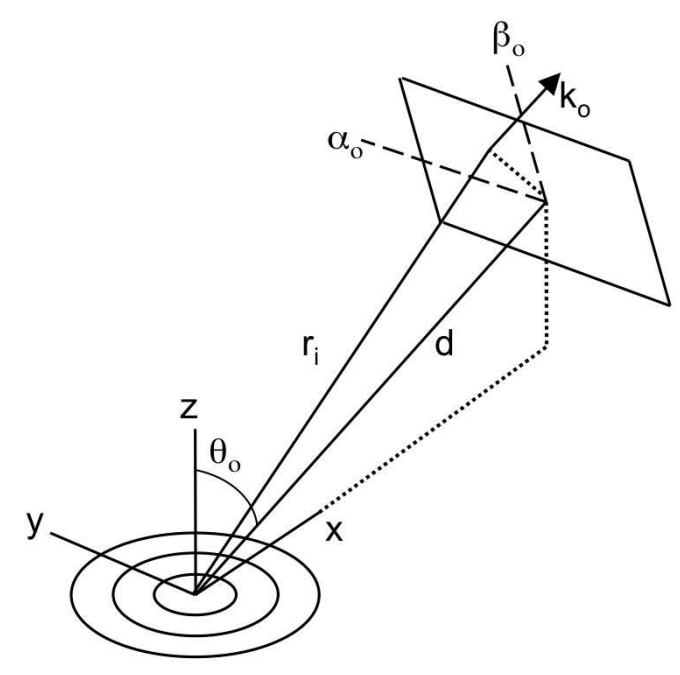

Figure 5.1 The geometry of ray tracing.

and test whether its value remains equal to $\xi=-1$ along each geodesic.

Starting from a fine raster of points on the image plane of an observer at infinity, I follow the geodesics backwards to the surface of the compact object or to different regions in the accretion flow where the photons originate.

Following Johannsen \& Psaltis (2010b), I consider an observer viewing the central object from a large distance $d$ and at an inclination angle $\theta_{o}$ from its rotation axis (see Fig. 5.1). I set up a virtual image plane that is perpendicular to the line of sight and centered at $\phi=0$ of the spacetime.

I define the set of Cartesian coordinates $\left(\alpha_{0}, \beta_{0}\right)$ on the image plane such that the $\beta_{0}$-axis is along the same fiducial plane and the $\alpha_{0}$-axis is perpendicular to it. I then convert the coordinates $\left(\alpha_{0}, \beta_{0}\right)$ of a photon that reaches the image plane to the coordinates $\left(r_{i}, \theta_{i}, \phi_{i}\right)$ in the spherical-polar system used for the metric (2.13) 
with the relations (see Johannsen \& Psaltis 2010b)

$$
\begin{aligned}
r_{i} & =\left(d^{2}+\alpha_{0}^{2}+\beta_{0}^{2}\right)^{1 / 2} \\
\cos \theta_{i} & =\frac{1}{r_{i}}\left(d \cos \theta_{o}+\beta_{0} \sin \theta_{o}\right) \\
\tan \phi_{i} & =\alpha_{0}\left(d \sin \theta_{o}-\beta_{0} \cos \theta_{o}\right)^{-1} .
\end{aligned}
$$

The photons that contribute to the image of the compact object are those with 3-momenta that are perpendicular to the image plane. This orthogonality condition uniquely specifies the momentum vector of a photon with the above coordinates, according to the relations (Johannsen \& Psaltis 2010b)

$$
\begin{aligned}
k^{r} & \equiv \frac{d r}{d \lambda^{\prime}}=\frac{d}{r_{i}} \\
k^{\theta} & \equiv \frac{d \theta}{d \lambda^{\prime}}=\left[-\cos \theta_{o}+\frac{d}{r_{i}^{2}}\left(d \cos \theta_{o}+\beta_{0} \sin \theta_{o}\right)\right] \\
k^{\phi} & \equiv \frac{\left.r_{i}^{2}-\left(d \cos \theta_{o}+\beta_{0} \sin \theta_{o}\right)^{2}\right]^{-1 / 2}}{d \lambda^{\prime}}=\frac{-\alpha_{0} \sin \theta_{o}}{\left(d \sin \theta_{o}-\beta_{0} \cos \theta_{o}\right)^{2}+\alpha_{0}^{2}} .
\end{aligned}
$$

Using these relations, I then calculate the $t$-component of the photon 4-momentum from equation (5.7). At this point, the normalization of the photon 4-momentum is arbitrary. Note that, for a distant observer, $d / r_{i} \rightarrow 1$, which implies that special care needs to be taken in evaluating expressions (5.13) and (5.14) to avoid round-off errors.

In the numerical algorithm, I integrate equations (5.3)-(5.6) using a fourthorder Runge-Kutta integrator. Figure 5.2 shows the convergence of my algorithm, for two cases. In the first case, I employ a fixed step $f$ in the affine parameter. In the second case, I employ an adaptive stepsize that I set equal to a fixed fraction 


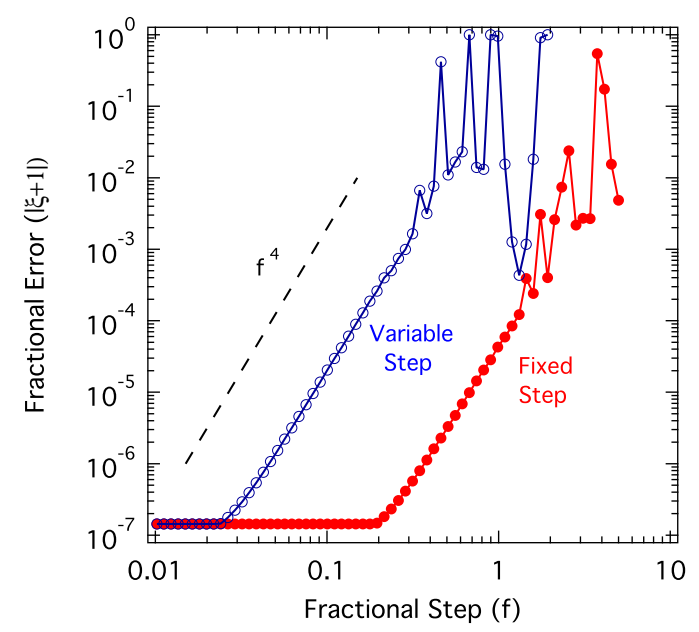

Figure 5.2 The fractional error introduced by the integration algorithm during the tracing of a single ray from the image plane to the equatorial plane, as a function of the fractional stepsize of the integration. The image plane is set at a distance of $1028 M$ and at an inclination of $40^{\circ}$. The black hole spin is equal to $a / M=0.5$ and its spacetime is described by the Kerr metric. The Cartesian coordinates of the position of the ray on the image plane are $\left(\alpha_{0}, \beta_{0}\right)=(5,0)$. The results of two integrations are shown, one with a fixed step in the affine parameter and one with a variable step. In both cases, the performance of the algorithm is consistent with the fourth-order of the integration method.

$f$ of the inverse rate of the fastest changing variable at each point, i.e.,

$$
\delta \lambda^{\prime}=f \min \left[t\left(\frac{d t}{d \lambda^{\prime}}\right)^{-1}, r\left(\frac{d r}{d \lambda^{\prime}}\right)^{-1}, \theta\left(\frac{d \theta}{d \lambda^{\prime}}\right)^{-1}, \phi\left(\frac{d \phi}{d \lambda^{\prime}}\right)^{-1}\right]
$$

In both cases, the algorithm shows the expected fourth-order convergence of my integration method. For very small values of the step size, the fractional error stabilizes and reflects the numerical accuracy to which the parameter $\xi$ was calculated on the image plane. For the calculations shown hereafter, I will use an adaptive step with $f=1 / 32 \simeq 0.03$.

My numerical algorithm is capable of integrating $\simeq 10^{4}$ geodesics in a time comparable to a second, on a personal computer with a $2.5 \mathrm{GHz}$ Intel core. This is 
comparable to the speed of other similar algorithms that employ different methods (e.g., Dexter \& Agol 2009; Doelence et al. 2009). Moreover, the algorithm is trivially parallelizable and, because of its very low storage requirements, is optimal for implementation on a GPU. 


\subsection{Relativistically Broadened Fluorescent Lines Around Quasi-Kerr Black Holes}

As a first application of my numerical algorithm, I calculate the profiles of relativistic broadened fluorescent iron lines from geometrically thin accretion disks around quasi-Kerr black holes. These are prime targets for current and future X-ray telescopes and are expected to lead to the measurements of the spins of a large number of black holes in binary systems and in active galactic nuclei (for reviews see Reynolds \& Nowak 2003; Miller 2007).

For this application, I trace rays from the image plane to the equatorial plane, where I will assume that a geometrically thin disks exists, from some outer radius $r_{\text {out }}$ down to the radius of the ISCO.

The character of the ISCO in the metric (2.13) depends on the sign and magnitude of the quadrupole deviation parameter $\epsilon$. When $\epsilon>0$, orbits close to the central object become unstable to radial perturbations. Following Johannsen \& Psaltis (2011b), I calculate the location of the ISCO in this case by finding the zero in the radial profile of the square of the radial epicyclic frequency for a particle in a circular equatorial orbit. For values of the quadrupole deviation parameter $\epsilon$ that are sufficiently negative, all circular equatorial orbits become stable to radial perturbations. However even in this case, orbits very close to the central object become unstable to vertical perturbations (see also Gair et al. 2008). Presently, I consider only positive deviations of the quadrupole moment of the metric from the Kerr value, i.e., $\epsilon>0$. When $\epsilon=0$, I use the complete expression for the location of the ISCO from Bardeen et al. (1973).

I assume that the disk is composed of a set of equatorial concentric rings, in which the plasma is moving at the local Keplerian velocity with (Glampedakis \& 


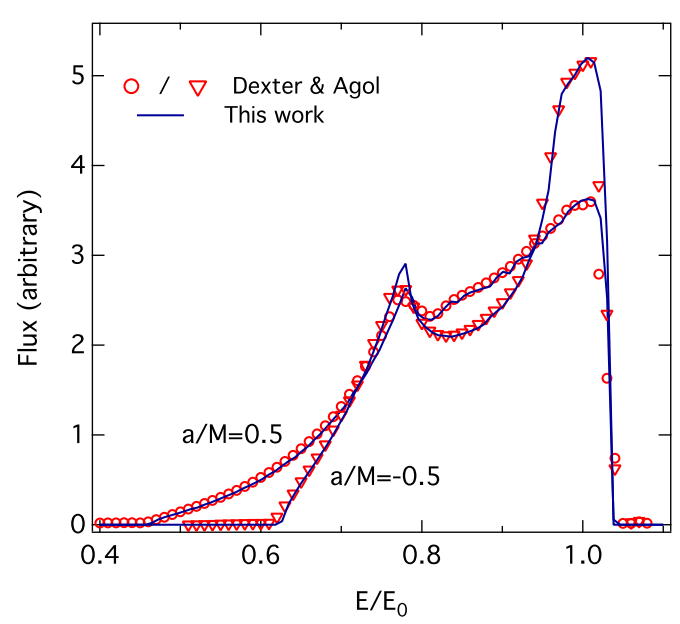

Figure 5.3 Profiles of relativistically broadened fluorescent lines from a geometrically thin accretion disk around a Kerr black hole, for two values of the black-hole spin. The outer radius of the disk is set to $15 M$, the power-law index of the line emissivity to $\alpha=2$, and the inclination to the observer to $\theta_{\mathrm{o}}=30^{\circ}$. The symbols are the results reported by Dexter \& Agol (2009), while the solid lines are the profiles calculated with the algorithm described here.

Babak 2006)

$$
\begin{aligned}
u^{\phi} & =\frac{1}{\Delta}\left[\frac{2 M}{r}(a E-L)+L\right]-\epsilon \frac{h_{3}}{r^{2}} L \\
u^{t} & =\frac{1}{\Delta}\left[E\left(r^{2}+a^{2}\right)+\frac{2 M a}{r}(a E-L)\right]-\epsilon f_{3} E\left(1-\frac{2 M}{r}\right)^{-1} .
\end{aligned}
$$

Here, $E$ and $L$ are the energy and angular momentum of the circular orbit at radius $r$ and are given in Johannsen \& Psaltis (2010a).

I also assume that radiation emerges from the accretion disk surface isotropically with an "emissivity" that scales as the power-law function $r^{-\alpha}$ of the coordinate radius. I calculate the overall change in the energy of the photon from the location of emission at the accretion disk to the image plane using

$$
\frac{E_{\mathrm{im}}}{E_{\mathrm{d}}}=\frac{g_{\mu \nu, \mathrm{im}} k_{\mathrm{im}}^{\mu} u_{\mathrm{im}}^{\nu}}{g_{\mu \nu, \mathrm{d}} k_{\mathrm{d}}^{\mu} u_{\mathrm{d}}^{\nu}}
$$




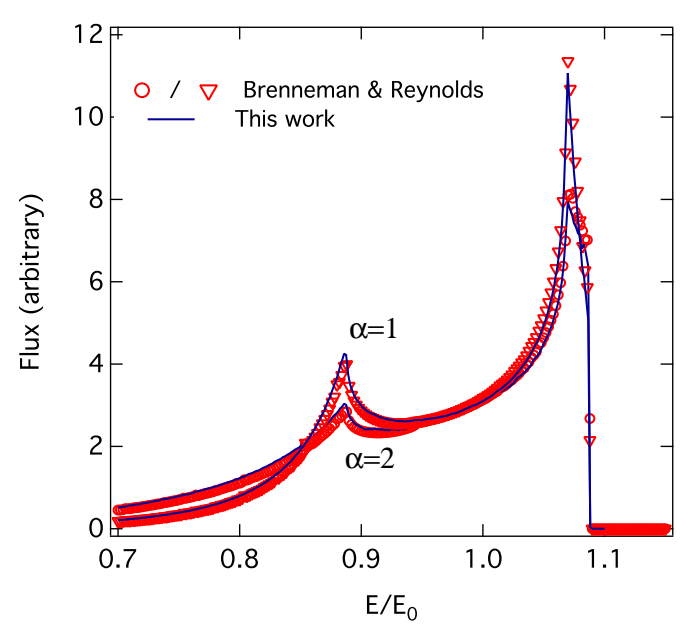

Figure 5.4 Profiles of relativistically broadened fluorescent lines from a geometrically thin accretion disk around a Kerr black hole, for two values of the line emissivity. The black hole spin is equal to $a / M=0.5$, the outer radius of the disk is set to $50 \mathrm{M}$, and the inclination to the observer to $\theta_{\mathrm{o}}=40^{\circ}$. The symbols are the results reported by Brenneman \& Reynolds (2006), while the solid lines are the profiles calculated with the algorithm described here.

and setting the 3-velocity of the observer at the image plane to zero. In this last expression, the subscripts "im" and " $\mathrm{d}$ " refer to the image plane and the accretion disk, respectively. I finally employ the Lorentz invariant quantity $I / E^{3}$, where $I$ is the monochromatic specific intensity of the radiation field and $E$ is the photon energy, in order to calculate the specific intensity at each point on the image plane.

The result at the completion of this calculation is the overall redshift or blueshift experienced by a photon that reaches each point on the image plane, which I denote by $g\left(\alpha_{0}, \beta_{0}\right) \equiv E_{\mathrm{im}} / E_{\mathrm{d}}$, and the corresponding specific intensity $I\left(\alpha_{0}, \beta_{0}\right)$. The monochromatic flux at the image plane is then

$$
F_{E} \sim \frac{1}{d^{2}} \int d \alpha_{0} \int d \beta_{0} I\left(\alpha_{0}, \beta_{0}\right) \delta\left[E-E_{0} g\left(\alpha_{0}, \beta_{0}\right)\right] .
$$

The presence of the $\delta$-function allows us in principle to convert this 2-dimensional 


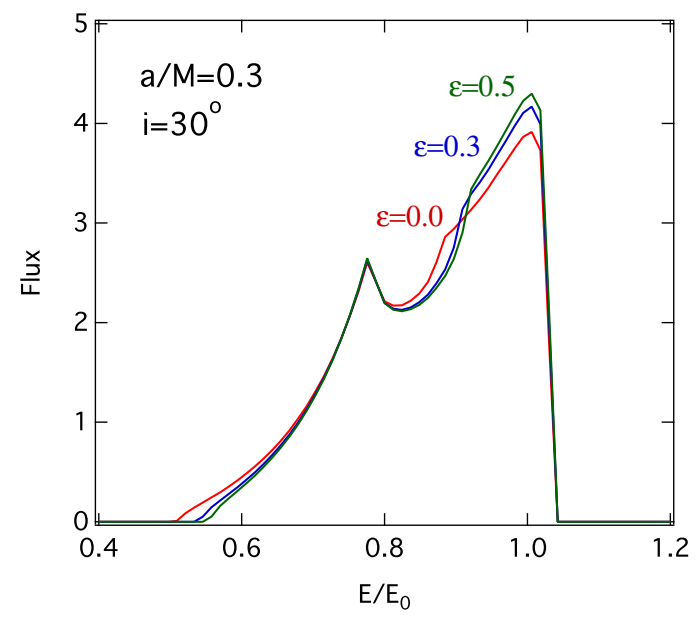

Figure 5.5 Profiles of relativistically broadened fluorescent lines from a geometrically thin accretion disk around a quasi-Kerr black hole, for a spin of $a / M=0.3$ and three values of the quadrupole deviation parameter $\epsilon$. The remaining parameters are the same as in Figure 5.3.

integral into a one-dimensional line integral along contours of constant values of the quantity $g\left(\alpha_{0}, \beta_{0}\right)$ on the image plane. In practice, calculating the location of these contours is time consuming. Instead, I evaluate expression (5.19) using a Monte Carlo integration of points on the image plane and a set of fine bins in photon energy.

Figures 5.3 and 5.4 show the dependence of the line profiles on the spin of the black hole and on the emissivity of fluorescence on the accretion disk. They also compare my results with other calculations from the literature, demonstrating the agreement between the different numerical algorithms. Figure 5.5 shows the dependence of the fluorescent line profiles on the parameter $\epsilon$, which measures the deviation of the quadrupole moment of the black-hole spacetime from its Kerr value. As I increase the degree of quadrupole deviation, the red wing of the line becomes less pronounced and the relative strengths of the blue and red wings is 
altered. Both effects are predominantly caused by the increase in the radius of the ISCO with increasing value of the parameter $\epsilon$. 


\subsection{Conclusions}

I described a new ray tracing algorithm for the calculation of observables from compact objects with spacetimes characterized by arbitrary quadrupole moments. Such spacetimes are relevant to black holes that violate the no-hair theorem and to moderately spinning neutron stars. I put special care in streamlining and accelerating my algorithm in order to achieve the efficiency neccessary for large parameter studies and comparisons to data. I also demonstrated the expected convergence of my algorithm and verified my results against those of previous calculations for Kerr metrics.

As a first application, I calculated the profiles of fluorescent iron lines from black holes that violate the no-hair theorem. As expected, varying the quadrupole moment of the spacetime, led to changes in the detailed profiles of the lines. I will study the observability of these effects as well as ways of breaking the degeneracy between changing the spin and the quadrupole of the black hole in Chapter 9. 


\section{CHAPTER 6}

\section{BlACK HOLE IMAGES}

In this chapter, I study the properties of the images of compact objects that violate the no-hair theorem using the quasi-Kerr formalism I developed in Chapter 2. I calculate numerically the mapping between locations in the vicinity of a black hole and positions in the observer's sky using the mass, spin, and quadrupole moment of the spacetime as independent parameters. I investigate the impact of varying the quadrupole moment on the properties of this mapping and show that the images of the accretion flows around compact objects that violate the no-hair theorem are expected to have prolate or oblate geometries.

I use my formalism to show that the bright emission ring surrounding the shadow of a black hole is circular for a Schwarzschild black hole and remains nearly circular for a Kerr black hole. On the other hand, if the quadrupole moment is left as an independent parameter, the ring shape changes significantly and becomes asymmetric. The degree of asymmetry is a direct measure of the violation of the no-hair theorem. I show that the diameter of the ring depends only very weakly on the spin and quadrupole moment of the black hole and can be used to directly measure the mass of the object. In addition, the ring is displaced off center in the image plane in the case of rotating black holes (Beckwith \& Done 2005; see, also, Takahashi 2004), and I show that the displacement is a direct measure of the object's spin, modulo the disk inclination. The contents of this chapter were published in Johannsen \& Psaltis (2010b, 2011a). See Psaltis \& Johannsen (2011) and Johannsen (2012) for reviews. 


\subsection{Testing the No-Hair Theorem with Black-Hole Images}

The no-hair theorem establishes the claim that astrophysical black holes are uniquely characterized by their mass $M$ and spin $J$, i.e., by only the first two multipole moments of their exterior spacetimes (Israel 1967, 1968; Carter 1971, 1973; Hawking 1972; Robinson 1975). As a consequence of the no-hair theorem, all higher order moments are already fully determined and obey the relation (2.5) (Geroch 1970; Hansen 1974)

This theorem is based on two technical assumptions. First, any spacetime singularity must be enclosed by an event horizon (the cosmic censorship conjecture, Penrose 1969), and second, there exist no closed timelike loops in the exterior domain of the black hole.

The no-hair theorem can be tested by measuring (at least) three different multipole moments, which have to be related by expression (2.5) if this theorem is correct (Ryan 1995). In Chapter 2, I investigated a framework for extracting three multipole moments of a black-hole spacetime with observations in the electromagnetic spectrum. I used a quasi-Kerr metric (Glampedakis \& Babak 2006), which incorporates an independent quadrupole moment given by expression (2.10) and parameterizes a potential deviation from the Kerr quadrupole in terms of the parameter $\epsilon$. This reduces smoothly to the Kerr quadrupole moment in the limit $\epsilon \rightarrow 0$ in accordance with relation (2.5).

If the no-hair theorem is valid, then $\epsilon=0$. If, however, a nonzero value of the parameter $\epsilon$ is measured, then the compact object cannot be a general-relativistic black hole. Within general relativity, it may be a different type of star or an exotic configuration of matter (see Collins \& Hughes 2004; Hughes 2006). On the other hand, if the compact object is otherwise known to possess an event horizon and 
a regular spacetime, then a nonzero value of the parameter $\epsilon$ implies that the nohair theorem is incorrect and general relativity does not accurately describe the near-horizon spacetimes of black holes.

In Chapter 2, I showed that the observable properties of this quasi-Kerr spacetime depend significantly on the parameter $\epsilon$ in the vicinity of the black hole. In particular, I demonstrated that the radius of the ISCO increases by $\sim 20 \%$ for a value of the quadrupolar correction $\epsilon=0.5$ at a spin $a=0.4 M$. Since the ISCO marks the inner edge of the accretion disk, it critically impacts the high-energy part of the emitted disk spectrum. Furthermore, I showed that the circular photon orbit experiences a shift of similar magnitude and that the observed redshift of a photon emitted by a particle on the ISCO decreases by $\sim 25 \%$ for values of the quadrupolar parameter $\epsilon=1.0$ and spin $a=0.4 M$, respectively. In addition, I demonstrated the effects of changing the quadrupole moment on the strong lensing experienced by photons in the neighborhood of the black hole.

For nonzero values of the parameter $\epsilon$, the quasi-Kerr metric is a solution of the Einstein equations up to the quadrupole order and, therefore, can only be used for values of the spin $a \lesssim 0.4 M$ and of the radius larger than a cutoff that depends on both the spin and the quadrupole moment. For reasonable perturbations of the quadrupole, however, the cutoff always lies inside of the circular photon orbit and plays only a very minor role in my analysis. The requirement that the spin is not near the maximal Kerr value allows us to apply this method to images of Sgr $\mathrm{A}^{*}$, for which first, although uncertain estimates from millimeter VLBI observations indicate that the black hole is not spinning rapidly (Broderick et al. 2009a). 


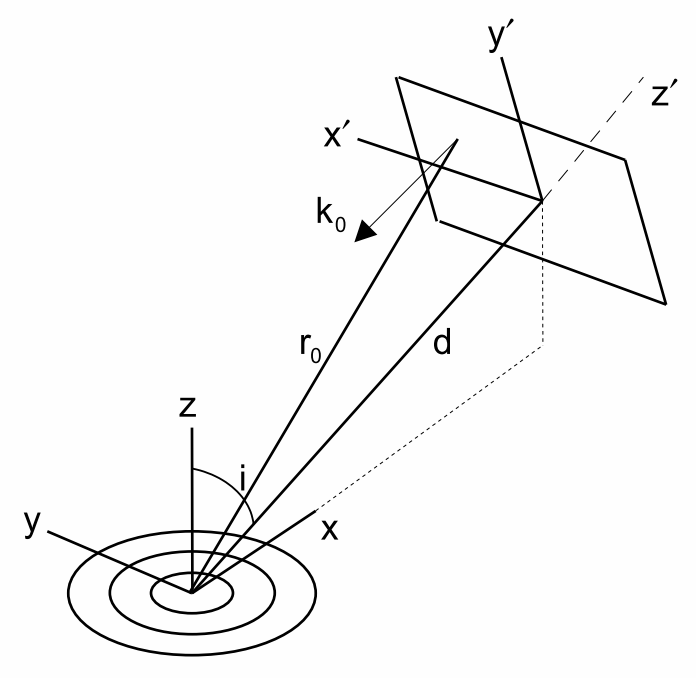

Figure 6.1 The geometry I used for simulating black-hole images, as projected on a Euclidean space. The black hole is located at the origin of the Cartesian coordinate system $(x, y, z)$. The image plane of a distant observer is located at a distance $d$ from the black hole, at an inclination angle $i$, and with its center on the $x-z$ plane. Photon trajectories are integrated backwards starting on the image plane at coordinates $\left(x^{\prime}, y^{\prime}\right)$ with uniform initial momentum vector $\vec{k}_{0}=-k_{0} \hat{z}^{\prime}$ at a distance $r_{0}$ from the black hole.

\subsection{The Apparent Shape of Quasi-Kerr Black Holes}

In this section, I calculate numerically the mapping between different locations in the accretion flow around a quasi-Kerr black hole and the observer's sky and discuss its characteristics. This mapping depends significantly on the value of the parameter $\epsilon$ due to the modifications in the spacetime properties discussed in Chapter 2, such as their effects on the light bending and redshift experienced by photons, as well as on the location of the ISCO and the photon orbit.

I developed an algorithm that maps any initial configuration of photons around the black hole into the plane of the sky viewed by a distant observer. I integrate 
the full second-order geodesic equations in the quasi-Kerr spacetime via a fourthorder Runge-Kutta method with adaptive stepsize. Figure 6.1 shows the geometry I use. The image plane is located at a distance $d$ away from the black hole, at an inclination angle $i$, and with its center on the $x-z$ plane. I integrate photon trajectories backwards starting on the image plane and terminating at the surface of last scattering in the accretion flow. Modeling the thermodynamic properties of the accretion flow and integrating the radiative transfer equation is beyond the scope of this chapter. In this section, I assume for simplicity that the last scattering surface of the photons is in the equatorial plane around the black hole, on the surface of an infinitesimally thin accretion disk. In the next section, I will discuss the situation in which the accretion flow is optically thin, as is expected to be the case for Sgr $\mathrm{A}^{*}$ at sub-mm wavelengths.

As an initial condition, I distribute the photons in a square grid on the image plane with a spacing of $\Delta r^{\prime}=0.025 M$. I set their initial 3-momentum vectors $\vec{k}_{0}$ to be perpendicular to the image plane and uniform (see Appendix C). In order to visualize the mapping between the plane of the accretion disk and the observer's sky I consider a set of concentric equatorial target rings that extend from $r=2.6 \mathrm{M}$ to $r=7.6 \mathrm{M}$ in steps of $1 \mathrm{M}$. Since the images are scale-invariant with respect to the mass of the black hole, I express all physical quantities in units of mass. In all calculations I have imposed a cutoff at $r=2.6 \mathrm{M}$ in accordance with the range of validity of the metric (see Chapter 2), and I terminate the integration if a photon enters the excluded region $r<2.6 \mathrm{M}$. I plot on the image plane only those photons that reach one of the target rings in the accretion disk.

In Figure 6.2 I plot images of black holes with spin $a=0.4 M$ viewed from a distant observer at an inclination angle $\cos i=0.25$ for values of the quadrupolar 

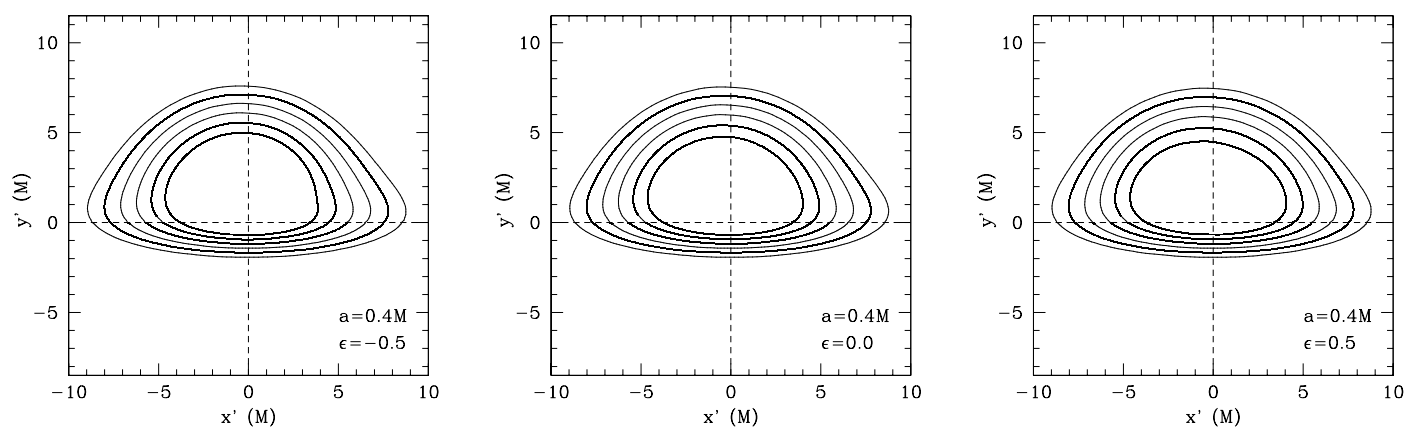

Figure 6.2 Concentric rings on the equatorial plane around a black hole, as seen by a distant observer located at an inclination of $\cos i=0.25$. The spacetime of the compact object has a spin of $a=0.4 M$ and a quadrupolar correction parameter (left) $\epsilon=-0.5$, (center) $\epsilon=0.0$, and (right) $\epsilon=0.5$. In all cases, frame dragging shifts the center of the images to the left. The effect of the quadrupolar correction is most apparent in the shape of the innermost ring. For $\epsilon=-0.5$, the innermost ring is more prolate compared to the Kerr black hole, while for $\epsilon=0.5$ it is more oblate.

parameter (from left to right) $\epsilon=-0.5,0.0,0.5$. The images consist of the projection of the target rings onto the observer's sky. The rings are distorted by light bending. Gravitational redshift, Doppler boosting, and beaming play no role in these images, because I have not incorporated in my calculation the evolution of the radiative intensity. In all images, the centers of the deformed rings are shifted to the left due to frame dragging.

The effect of changing the quadrupole moment on the images is only apparent at small radii. In Figure 6.3 I show explicitly how violating the no-hair theorem alters the images of the innermost rings I considered, i.e., the ones at $r=2.6 \mathrm{M}$, for a Schwarzschild black hole and a Kerr black hole with $a=0.4 M$. In both cases, I set the inclination to $\cos i=0.25$ and display the innermost ring for three values of the parameter $\epsilon=-0.5,0.0,0.5$. For the perturbed Schwarzschild black hole, 

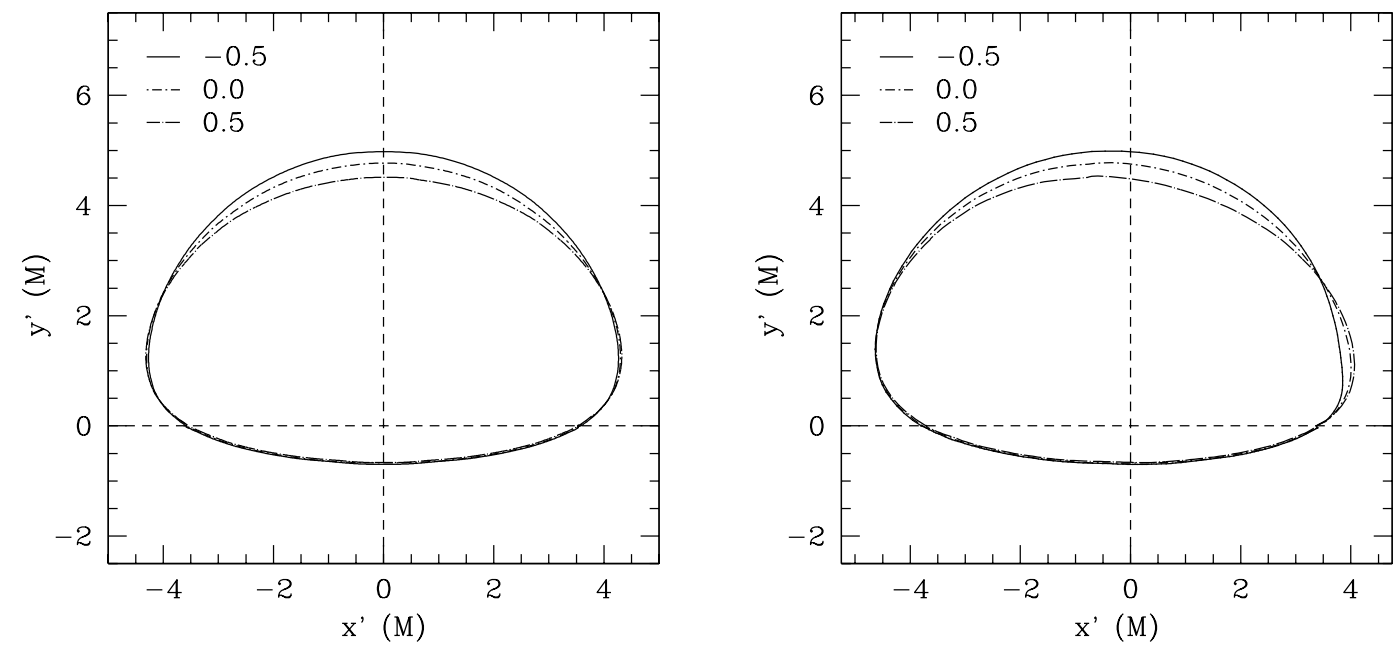

Figure 6.3 Images of the innermost rings shown in Figure 6.2 for three values of the parameter $\epsilon$. The top panel corresponds to a Schwarzschild black hole, whereas the bottom panel corresponds to a Kerr black hole with $a=0.4 M$. The inclination angle is set to $\cos i=0.25$ in both cases.

increasing the value of the parameter $\epsilon$ makes the projected ring more oblate, because of the increased amount of gravitational lensing experienced by the photons (see Chapter 2). In the case of nonzero spin, a change in the quadrupole moment also affects the left-right asymmetry of the image and especially its right edge that corresponds to the receding part of the accretion disk. Increasing the value of the parameter $\epsilon$ leads to a wider image. 


\subsection{Photon Rings}

The dependence of the black-hole images on their quadrupole moments that I investigated in the previous section allows us, in principle, to use imaging observations to map the properties of their spacetimes. In practice, however, the change in the oblateness of even the closest rings to the black-hole horizons is very modest and can be masked by anisotropies in the emission from the accretion flow and its variability. Unless an orbiting density inhomogeneity (a "hot spot") $^{\prime \prime}$ can be securely identified and imaged throughout its orbit (e.g., Broderick \& Loeb 2005), time-averaged overall images of black-hole accretion disks may appear a priori not well suited for testing the no-hair theorem. There is, however, an observable structure in the images of optically thin accretion flows around black holes that suffers only marginally from astrophysical complications and carries very strong signatures of the quadrupole moments of the underlying spacetimes.

Consider a geometrically thick accretion flow imaged at a wavelength at which the emission is optically thin; this is the case for Sgr $\mathrm{A}^{*}$ at sub-mm wavelengths. The brightness of the image at any given point in the observer's sky will depend on the length of the optical path along the corresponding light ray that passes through the region of high emissivity in the accretion flow. Most light rays that originate from the image plane either emerge on the far side of the black hole, after experiencing gravitational bending, or intercept the black-hole hori-

zon. A small set of light rays, however, that approach the event horizon orbit closely around the black hole several times before they escape towards the far side and can, therefore, make a significant contribution to the total flux (Cunningham 1976; Laor, Netzer, \& Piran 1990; Viergutz 1993; Bao, Hadrava, \& Østgaard 1994; Čadež, Fanton, \& Calvani 1998; Agol \& Krolik 2000; Beckwith \& Done 2005). 
This is illustrated in Figure 6.4, which shows the paths of a number of light rays that approach the horizon of a black hole from the top right corner at an inclination of $\cos i=0.25$ relative to the spin axis $z$. These light rays orbit several times around the black-hole horizon at nearly constant radius (see, also, Bardeen 1973).

The integral of the emissivity along these light rays is very large, compared to that along nearby light rays, and results in a significant increase in the brightness at their footpoints on the image plane. The locus of the footpoints of these light rays on the image plane is a ring. This leads to the emergence of a ring in images of optically thin accretion flows that is substantially brighter than the background and whose shape and position in the image plane depend on the spin of the black hole and on the inclination of the accretion disk (Beckwith \& Done 2005). Such bright rings of emission are clearly visible in the images of all time-dependent general relativistic simulations of accretion flows reported to date (see, e.g., the right panel of Fig. 5 in Mościbrodzka et al. 2009; panels 1 and 3 in Fig. 1 in Dexter, Agol, \& Fragile 2009; Fig. 1 in Shcherbakov \& Penna 2010).

In this section, I demonstrate that the bright rings of emission (hereafter photon rings) carry an unmistakable signature of the black-hole spacetime and, in particular, of its quadrupole moment. In order to study the sizes and shapes of these rings in different configurations, in a way that is independent of the physical conditions in the accretion flow, I use the following working definition of the position of the bright photon rings on the image plane: I identify them with the locus of footpoints of those light rays that leave the observer perpendicular to the image plane and cross the equatorial plane of the black hole at least twice, before emerging on the opposite side.

I first consider the dependence of the position of the photon rings on the spin 


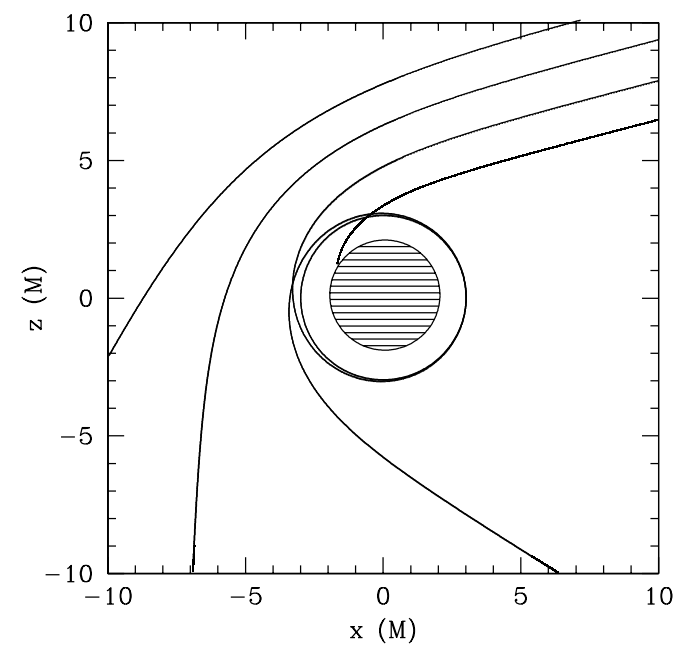

Figure 6.4 Light rays around a Schwarzschild black hole illustrating the emergence of the bright emission ring. Several light rays approach the black hole from the top right corner. If a ray reaches the photon ring with a 3-momentum that is nearly tangential to the photon orbit, it orbits around the black hole several times, while all other rays are either immediately scattered or captured by the black hole. The footpoints of the orbiting light rays on the image plane will be brighter than those of the nearby rays. The shaded region marks the event horizon.

of a Kerr black hole, i.e., of one that satisfies the no-hair theorem. Figure 6.5 shows that increasing the spin leads to a substantial displacement of the centroid of the photon ring with respect to the geometric center of the spacetime. This displacement is $\simeq 1 M$ for even moderate $(a=0.5 M)$ values of the spin and can be as large as $\simeq 2 M$ for maximally rotating black holes. The striking property of these rings, however, is the fact that their shapes remain practically circular for values of the spin $a \lesssim 0.9 M$, even though the geometry of the Kerr spacetime is highly non-spherically symmetric. Only at the maximum spin, the receding part of the photon ring becomes asymmetric. Even in this extreme case, however, the max- 


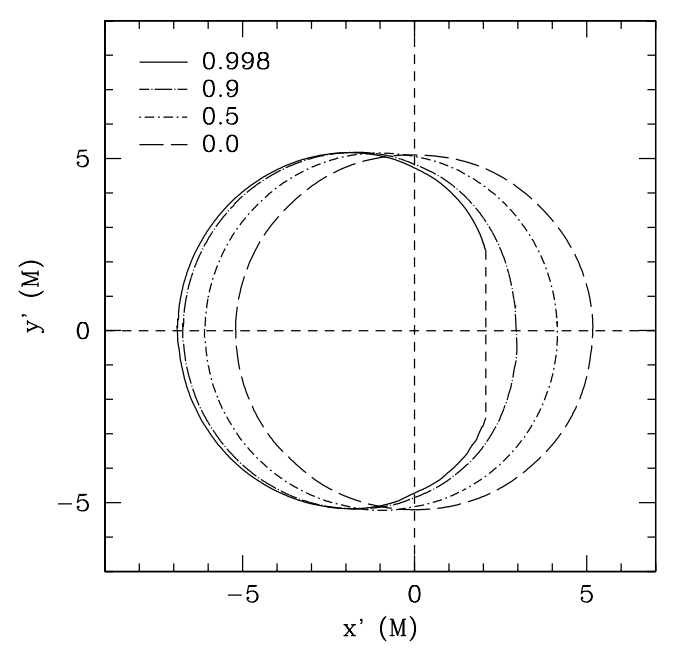

Figure 6.5 The dependence of the bright photon ring seen by a distant observer on the spin of a Kerr black hole. Increasing the spin leads to a displacement of the photon ring with minimal deformation of its shape. In all cases, the inclination of the observer corresponds to $\cos i=0.25$.

imum difference between the major and minor axes of the ring is only $\simeq 1.5 \mathrm{M}$. Note here that, for the case $a=0.998 M$, the radius of the circular photon orbit almost coincides with the event horizon, which introduces a noticeable numerical error on the rightmost part of the ring; for this reason, I use a short-dashed line to represent this uncertainty.

The symmetry of the photon rings changes significantly if the no-hair theorem is violated and the quadrupole moment of the spacetime takes on a non-Kerr value. In Figure 6.6, I show the photon rings for a (quasi-)Schwarzschild and a (quasi-)Kerr black hole with spin $a=0.4 M$ and for three different values of the quadrupolar correction $\epsilon=-0.5,0.0,0.5$. As the degree of violation of the no-hair theorem increases, the photon ring around a static black hole becomes 
oblate or prolate (depending on the sign of the parameter $\epsilon$ ), while its geometric center remains the same. In the case of a spinning black hole, frame dragging introduces an additional asymmetry between the approaching and the receding parts of the ring. For the spinning black holes with a value of the quadrupolar parameter $\epsilon=-0.5$, the rightmost part of the ring corresponds to photons that propagate in a region of spacetime in which my perturbative approach breaks down; I, therefore, indicated this uncertainty using a long-dashed line.

In both cases shown in Figure 6.6, the difference between the major and minor axes of the photon rings is $\simeq 1-2 M$, even for modest degrees of violation of the no-hair theorem. The degree of asymmetry increases with increasing inclination and vanishes when the black hole is viewed pole on. The oblateness and asymmetry of the bright photon rings in the images of accreting black holes carries, therefore, a quantitative measure of the degree of violation of the no-hair theorem, modulo the inclination of the observer. I systematically analyze the impact of the mass, spin, quadrupolar parameter, and disk inclination on the shape and position of the ring in the next section. 

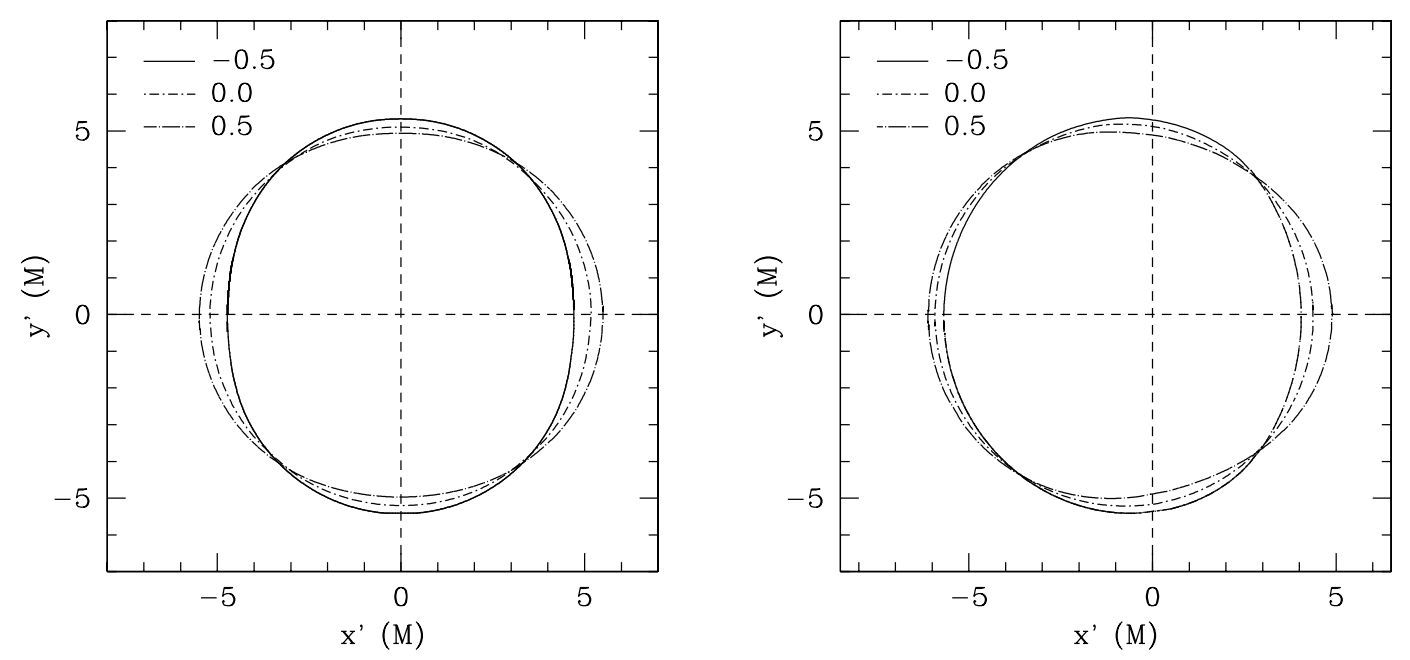

Figure 6.6 The dependence of the bright photon rings on the degree of violation of the no-hair theorem, parametrized by the difference $\epsilon$ between the quadrupole moment of the spacetime and its Kerr value. The top panel corresponds to a Schwarzschild black hole whereas the bottom panel corresponds to a Kerr black hole with $a=0.4 M$. In both cases, the inclination of the observer is $\cos i=0.25$. Violation of the no-hair theorem causes the ring to become ellipsoidal, if the black hole is static, or even more asymmetric, if the black hole is rotating.

\subsection{Photon Ring Properties}

In this section, I analyze in detail the dependence of the image of the photon ring for a given black hole on its mass $M$, spin $a$, disk inclination angle $i$, and quadrupolar correction parameter $\epsilon$.

In order to quantify the effect of changing this set of parameters on the shape and location of a photon ring in the image plane, I define the displacement and the asymmetry of the ring in the following way. First I define the horizontal displacement $D$ of the ring by the expression

$$
D \equiv \frac{\left|x_{\max }^{\prime}+x_{\min }^{\prime}\right|}{2},
$$


where $x_{\max }^{\prime}$ and $x_{\min }^{\prime}$ are the maximum and minimum abscissae of the ring in the image plane, respectively. Due to the reflection symmetry across the equatorial plane there is no displacement in the vertical direction, whereas in the horizontal direction the displacement can be as large as $D \simeq 2 M$ for rapidly spinning black holes.

Next, I define the average radius $<R>$ of the ring by the expression

$$
<R>\equiv \frac{\int_{0}^{2 \pi} R \mathrm{~d} \alpha}{\int_{0}^{2 \pi} \mathrm{d} \alpha}
$$

where

$$
R \equiv \sqrt{\left(x^{\prime}-D\right)^{2}+y^{\prime 2}}
$$

and

$$
\tan \alpha=\frac{y^{\prime}}{x^{\prime}} .
$$

I also define the ring diameter $L$ by the expression

$$
L \equiv 2<R>\text {. }
$$

Finally, I define the asymmetry $A$ of the ring image by the expression

$$
A \equiv 2 \sqrt{\frac{\int_{0}^{2 \pi}(R-<R>)^{2} \mathrm{~d} \alpha}{\int_{0}^{2 \pi} \mathrm{d} \alpha}} .
$$

I simulated ring images for various values of the spin $a$, inclination $i$, and the parameter $\epsilon$. In Figure 6.7, I plot the ring displacement $D$ for a Kerr black hole as a function of the inclination angle $i$ for different values of the spin. Triangles denote the data points obtained from my simulation.

I found that the displacement depends primarily on the spin and the disk inclination, but it depends only weakly on the quadrupolar parameter $\epsilon$. The displacement is well approximated by the expression

$$
D=2 a \sin i\left(1-0.41 \epsilon \sin ^{2} i\right),
$$




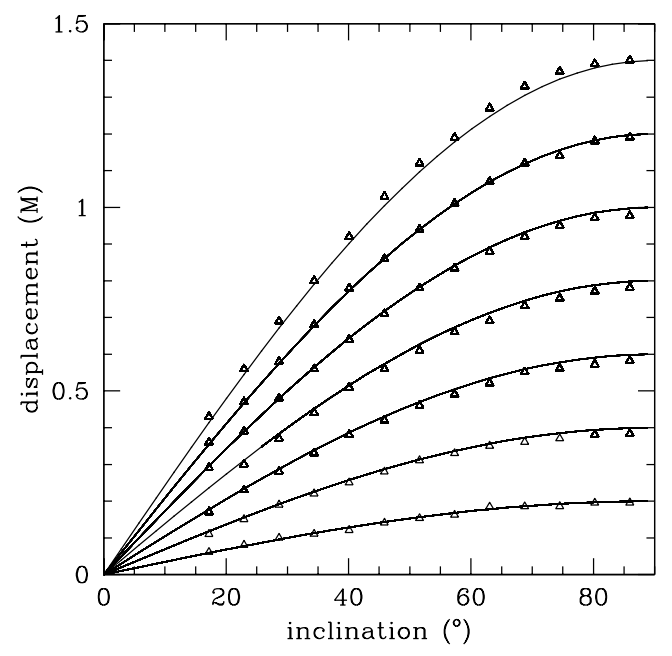

Figure 6.7 The ring displacement versus the disk inclination for a Kerr black hole with spins (top to bottom) $a=0.7 M, 0.6 M, \ldots, 0.1 M$. The solid lines show the functional form (6.7) with the parameter $\epsilon$ set to zero.

where $a \lesssim 0.7 M$ for a Kerr black hole whereas $a \leq 0.4 M$ and $0 \leq \epsilon \leq 0.5$ for a quasi-Kerr black hole. From my simulations I find a slight deviation for rapidly spinning Kerr black holes with a spin of $a \gtrsim 0.7 M$ leading to an additional displacement of $0.12 a^{2} \sin i$.

For a Kerr black hole, the dependence of the displacement on the spin and the inclination is reminiscent of the location of the caustics in Kerr spacetime (Rauch \& Blandford 1994; Bozza 2008; see also Bozza 2009 and references therein), and provides an independent check of the nature of the central object. Any deviation from this dependence necessarily implies that the compact object is not a Kerr black hole. For nonzero values of the parameter $\epsilon$, the displacement is only moderately affected. 

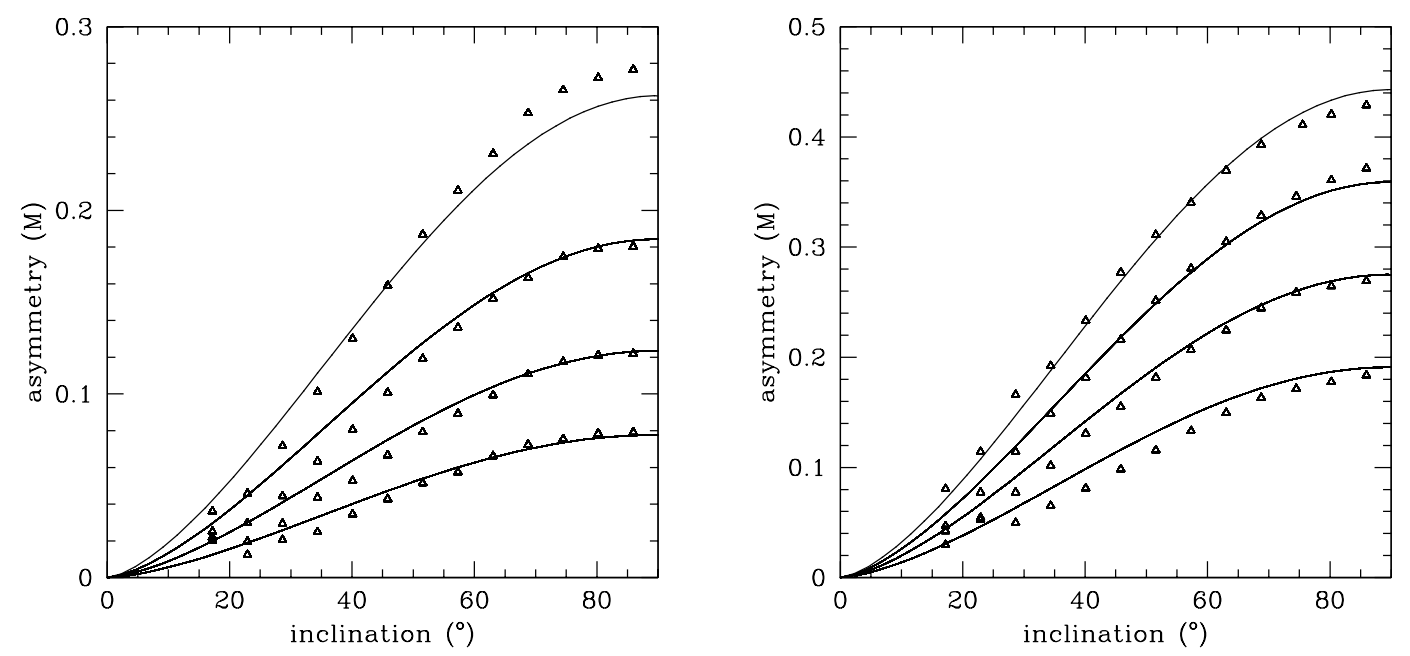

Figure 6.8 The asymmetry of the photon ring as a function of the inclination angle for (top) a Kerr black hole with values of the spin (top to bottom) $a=$ $0.9 M, 0.8 M, 0.7 M, 0.6 M$ and (bottom) a quasi-Kerr black hole with a spin of $a=0.4 M$ and values of the parameter (top to bottom) $\epsilon=0.5,0.4,0.3,0.2$. The solid lines show the functional form (6.8) that describes these simulations.

In Figure 6.8, I plot the ring asymmetry as a function of the inclination angle for (top panel) a Kerr black hole with different values of the spin and for (bottom panel) a quasi-Kerr black hole with a spin of $a=0.4 M$ and different values of the quadrupolar parameter $\epsilon$. In the case of a Kerr black hole, I find that the asymmetry is negligible for slowly spinning black holes. In the case of a quasiKerr black hole, the asymmetry scales linearly with the quadrupolar parameter $\epsilon$. I fit the asymmetry with the expression

$$
\frac{A}{M}=\left[0.84 \epsilon+0.36\left(\frac{a}{M}\right)^{3}\right] \sin ^{3 / 2} i
$$

which is valid for a Kerr with arbitrary spin and for a quasi-Kerr black hole with values of the spin $0.0 \leq a / M \leq 0.4$ and of the parameter $0.0 \leq \epsilon \leq 0.5$, respec- 
tively.

The asymmetry depends strongly on the quadrupolar correction parameter $\epsilon$ and the disk inclination $i$ whereas it depends only weakly on the spin as long as the black hole does not spin rapidly. Expression (6.8) implies that the maximum asymmetry of a Kerr black hole is $\simeq 0.36 M$. In the event that an asymmetry larger than this value is detected, which is already possible for moderate deviations of the quadrupole moment from the value of a Kerr quadrupole moment (c.f., Figure 6.8, bottom panel), then the compact object cannot be a Kerr black hole.

In order to test the no-hair theorem, it is necessary to measure (at least) three multipole moments of the spacetime (Ryan 1995). In the following I argue that a measurement of the diameter of the ring determines the mass of the central object and that for a given disk inclination the displacement and the asymmetry of the ring directly measure the spin $a$ and the parameter $\epsilon$, respectively. Therefore, the degree of asymmetry is a direct measure of the violation of the no-hair theorem.

In Figure 6.9, I plot (left panel) the diameter of the photon ring as a function of the spin $a$ for values of the inclination $17^{\circ} \leq i \leq 86^{\circ}$. Dashed lines correspond to a quasi-Kerr black hole with a value of the parameter $\epsilon=0.5$. The solid line corresponds to a Kerr black hole. In all cases, the diameter is practically independent of the spin $a$. For a Kerr black hole, the diameter also depends only very weakly on the disk inclination and is almost constant for spins in the range $0 \leq a / M \leq 0.4$ with a value of

$$
L=10.4 M
$$

Even for large values of the spin, the diameter depends only weakly on the inclination angle causing a systematic uncertainty of only $\simeq 2 \%$ for a value of the spin as large as $a=0.9 \mathrm{M}$. For a quasi-Kerr black hole, the diameter is affected 
by the parameter $\epsilon$ and the inclination which leads to a systematic uncertainty of $\simeq 5 \%$ if $\epsilon=0.5$. Therefore, a measurement of the ring diameter directly measures the mass of the central object.

In Figure 6.9, I also plot (center panel) the ring displacement of a quasi-Kerr black hole versus $a \sin i$ for values of the inclination angle $17^{\circ} \leq i \leq 86^{\circ}$ and of the parameter $0 \leq \epsilon \leq 0.5$. The solid lines in this figure represent the extreme limits of the fit function given by expression (6.7) corresponding to $\epsilon=0$ and $\epsilon=0.5, \sin i=1$, respectively. Finally, in Figure 6.9 (right panel), I show the ring asymmetry of quasi-Kerr black holes versus $\epsilon \sin ^{3 / 2} i$ for values of the spin $0 \leq a / M \leq 0.4$ and of the inclination angle $17^{\circ} \leq i \leq 86^{\circ}$. The solid line is expression (6.8) evaluated at $a=0.0 M$.

Thus, for a given disk inclination, an observation of the displacement and the asymmetry directly leads to a measurement of the spin $a$ and quadrupolar correction parameter $\epsilon$ via the expressions in equations (6.7) and (6.8), respectively. A measurement of the asymmetry allows for a test of the no-hair theorem. In order to determine the displacement it is necessary to measure the center of mass of the accretion flow around the black hole. An estimate of the location of the center of mass may perhaps be obtained from a (time-averaged) image of the outer edges of the accretion flow. 

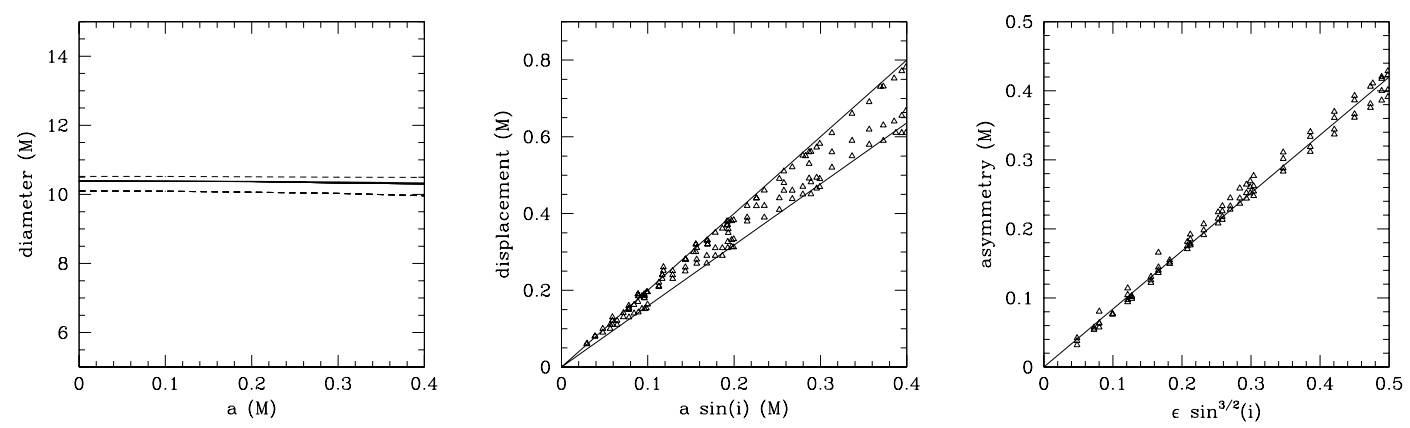

Figure 6.9 (Left:) The upper and lower limit of the ring diameter versus the spin for inclinations $17^{\circ} \leq i \leq 86^{\circ}$ for a Kerr black hole (solid lines) and for a quasiKerr black hole with a value of the parameter $\epsilon=0.5$ (dashed lines). The diameter is practically independent of the spin and has a constant value of $\simeq 10.4 M$ for a Kerr black hole. The dependence on the parameter $\epsilon$ and the disk inclination is weak. A measurement of the ring diameter is a direct measure of the mass. (Center:) The displacement of the photon ring as a function of $a \sin i$ for various values of the parameter $0 \leq \epsilon \leq 0.5$. The displacement is predominantly determined by the spin and the disk inclination allowing for a direct measurement of the quantity $a \sin i$. (Right:) The ring asymmetry versus $\epsilon \sin ^{3 / 2} i$ for various inclinations $17^{\circ} \leq i \leq 86^{\circ}$ and $0.0 \leq a / M \leq 0.4$. The asymmetry depends only weakly on the spin and hence provides a direct measure of the quantity $\epsilon \sin ^{3 / 2} i$.

\subsection{Conclusions}

In Chapter 2, I proposed a new framework for testing the no-hair theorem with observations of black holes in the electromagnetic spectrum. I formulated my tests based on a quasi-Kerr metric (Glampedakis \& Babak 2006), which deviates smoothly from the Kerr metric in the quadrupole moment. Since the no-hair theorem admits exactly two independent multipole moments for a black hole, a measurement of these three moments will allow us to test the no-hair theorem.

In this chapter, I calculated numerically the mapping between different locations in the accretion flow around a quasi-Kerr black hole and positions in the 
image plane of a distant observer. My calculations allowed us to study the potential of using imaging observations of black holes, that will become available in the near future, in order to test the no-hair theorem.

I argued that the expected image of an accretion flow will be characterized by a bright emission ring generated by light rays that circle multiple times around the event horizon before emerging towards the observer. I identified the ring diameter as a direct measure of the mass of the black hole, and I quantified the dependence of the displacement and the asymmetry of the ring on the spin and the quadrupolar parameter as well as on the disk inclination. For a given inclination angle, a measurement of the displacement and the asymmetry directly measures the spin and the quadrupolar parameter of the system, respectively. The asymmetry itself provides a direct measure of the violation of the no-hair theorem.

It is important to emphasize here that only the relative displacement and asymmetry of the ring (i.e., measured in units of the ring diameter) and not their absolute values are necessary in inferring the spin and quadrupole moment of the black-hole spacetime. As a result, the outcome of such an observation does not depend on the distance to the black hole, which might not be known accurately. On the other hand, the angular diameter of the photon ring is proportional to the mass of the black hole. This can lead to an accurate measurement of the mass of the black hole if the distance is known, or else of the distance to the black hole, if its mass is known from, e.g., dynamical observations.

Sgr $A^{*}$, the black hole in the center of the Milky Way, is the ideal candidate for a test of the no-hair theorem due to its high brightness, large angular size, and relatively unimpeded observational accessibility. Recent VLBI observations 
(Doeleman et al. 2008) resolved Sgr $A^{*}$ on horizon scales. Incorporating additional baselines to the VLBI network will lead to the first images of Sgr A* within the next few years (Fish \& Doeleman 2009). The emission from Sgr A* at sub$\mathrm{mm}$ wavelengths is optically thin, the size of the scattering ellipse is $\lesssim 1 M$, and the resolution of a VLBI image at this wavelength is also comparable to $\simeq 1 M$ (Doeleman et al. 2008; Fish \& Doeleman 2009). The smearing of the images due to scattering and the finite resolution of the array will, therefore, not preclude measuring the position and asymmetry of the photon ring to an accuracy that is adequate in providing a quantitative test of the no-hair theorem.

Observations of the orbits of stars in the vicinity of the black hole have provided an independent measurement of its mass (Ghez et al. 2008; Gillessen et al. 2009). Perhaps more importantly, the same observations may lead in the future to an independent measurement of the spin and orientation of the black hole, as well as to a complementary test of the no-hair theorem (Will 2008; Merritt et al. 2010). 


\section{CHAPTER 7}

\section{Masses of Nearby Supermassive Black Holes With Very-LONG BASELINE INTERFEROMETRY}

Sgr A*, the supermassive black hole at the center of our galaxy, has been observed for several decades. Monitoring stars orbiting around Sgr $\mathrm{A}^{*}$ has led to measurements of its mass and distance (Ghez et al. 2008; Gillessen et al. 2009). However, these measurements of mass and distance are strongly correlated. For purely astrometric measurements, mass and distance are related as $M \sim D^{3}$, while for measurements of radial velocities mass and distance are related as $M \sim D^{0}$. For combined data sets, the correlation between mass and distance behaves roughly as $M \sim D^{2}$ (Ghez et al. 2008; Gillessen et al. 2009). This correlation between mass and distance constitutes a major source of uncertainty in our understanding of the properties of Sgr $\mathrm{A}^{*}$. Likewise, dynamical measurements of the masses of a number of nearby supermassive black holes have been obtained with often much greater uncertainties (see, e.g., Gültekin et al. 2009).

Another technique, VLBI, aims to image Sgr A* directly. Recent VLBI observations with an array consisting of the Submillimeter Telescope Observatory (SMTO) in Arizona, the James Clerk Maxwell Telescope (JCMT) on Mauna Kea, and several of the dishes of the Combined Array for Research in Millimeter-wave Astronomy (CARMA) in California resolved Sgr A* on scales comparable to its event horizon and identified sub-horizon size structures (Doeleman et al. 2008; Fish et al. 2011). Images of accretion flows around black holes have the shadow of the compact object imprinted on them, which depends uniquely on its mass, spin, and inclination (e.g., Falcke et al. 2000) as well as on possible deviations from the 
Kerr metric (Johannsen \& Psaltis 2010). Based on such images and assuming the mass and distance obtained from the monitoring of stellar orbits, these VLBI observations inferred constraints on the inclination and spin of Sgr A* (Broderick et al. 2009, 2011) and placed limits on potential non-Kerr signatures (Broderick et al. 2012).

In addition to the shadow, images of optically thin accretion flows around black holes carry a characteristic signature in the form of a bright ring (Johannsen \& Psaltis 2010), which I refer to as the photon ring. Light rays that approach the event horizon closely orbit around the black hole many times before they are detected by a distant observer, resulting in a bright ring due to their long optical path length through the accretion flow. The flux of such photons can account for a significant fraction of the total disk flux and produce higher order images (Cunningham 1976; Laor, Netzer, \& Piran 1990; Viergutz 1993; Bao, Hadrava, \& Østgaard 1994; Čadež, Fanton, \& Calvani 1998; Agol \& Krolik 2000; Beckwith \& Done 2005). These photon rings are clearly visible in all time-dependent general-relativistic simulations of accretion flows that have been reported to date (Mościbrodzka et al. 2009; Dexter, Agol, \& Fragile 2009; Shcherbakov \& Penna 2010).

Johannsen \& Psaltis (2010) showed that a measurement of the ring diameter measures the ratio $M / D$ for the black hole, independent of its spin or deviation from the Kerr metric. Therefore, combining such a measurement with the observations of stars around Sgr A* can reduce the correlation between mass and distance.

In this chapter, I explore the ability of this approach to refine the mass and distance measurements of Sgr $\mathrm{A}^{*}$. I estimate the precision with which a ther- 
mal noise-limited VLBI array can infer the diameter of the ring of Sgr $\mathrm{A}^{*}$ and use a Bayesian technique to simulate measurements of the mass and diameter of Sgr $A^{*}$ in conjunction with parameters inferred from the existing data of the orbits of stars at comparable wavelengths. I show that, in this best-case scenario, the correlation between mass and distance is reduced significantly. In addition, I argue that the accretion flows of other nearby supermassive black holes are optically thin, allowing for VLBI observations of their respective photon rings. I assess the prospects of using this technique to infer the masses of these sources. The contents of this chapter have been submitted to The Astrophysical Journal as Johannsen et al. (2012a). 


\subsection{Measuring the Photon Ring of Sgr A*}

The properties of photon rings are practically independent of the specific flow geometry and remain constant even if the accretion flow itself is highly variable (Johannsen \& Psaltis 2010). The relative brightness as well as the constancy of these rings make them ideal targets for VLBI-imaging observations.

For a Kerr black hole with mass $M$, the shape of a given photon ring has a diameter of

$$
L \simeq 10.4 r_{g}
$$

which remains practically constant for all values of the spin and disk inclination (Johannsen \& Psaltis 2010). In this expression,

$$
r_{g} \equiv \frac{G M}{c^{2}}
$$

is the gravitational radius, and $G$ and $c$ are the gravitational constant and the speed of light, respectively.

The angular diameter $\theta_{\text {ring }}$ of the diameter of the photon ring of a black hole is given by the ratio of its diameter and distance,

$$
\theta_{\text {ring }}=\frac{L}{D} \text {. }
$$

Assuming the current mass and distance measurements of Sgr $\mathrm{A}^{*}, M_{0}=4.3 \times$ $10^{6} M_{\odot}$ and $D_{0}=8.3 \mathrm{kpc}$ (Gillessen et al. 2009), the photon ring has an angular diameter of

$$
\theta_{0} \simeq 53 \mu \text { arcsec. }
$$

Radio interferometers are limited by their intrinsic resolution as well as by interstellar scattering. In order to identify the range of wavelengths within which 


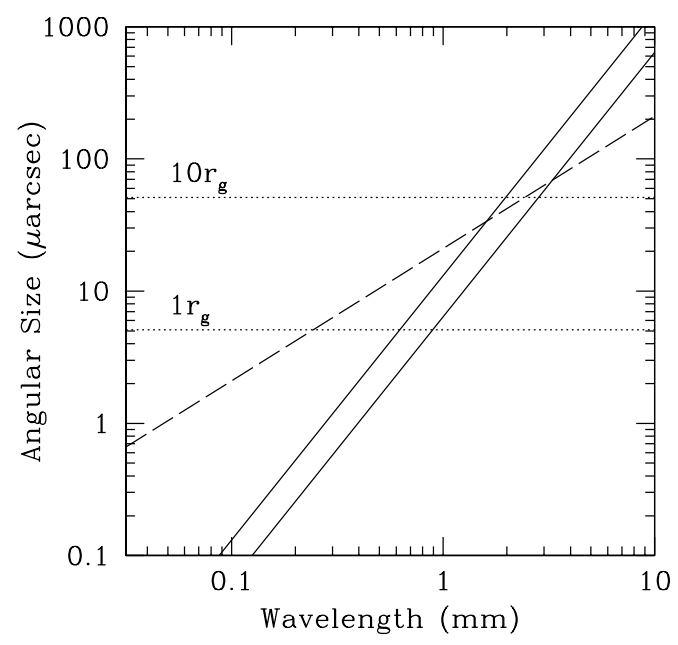

Figure 7.1 Solid lines: Degree of the blurring of structures on the image of Sgr $\mathrm{A}^{*}$, inferred using the interstellar scattering law of Bower et al. (2006); the two lines correspond to the major and minor axes of the scattering ellipse. Long dashed line: Resolution estimate of a radio interferometer, taken as $\theta_{\text {res }}=k \lambda / d$ with $k=1$ and $d=10^{4} \mathrm{~km}$. Dotted lines: Angular diameter of Sgr A* corresponding to length scales of $10 r_{g}$ and $1 r_{g}$, respectively. Imaging the photon ring of Sgr $\mathrm{A}^{*}$ with VLBI is possible at wavelengths $\lambda \lesssim 1 \mathrm{~mm}$.

VLBI measurements of the photon ring of Sgr $\mathrm{A}^{*}$ are resolution-limited, I compare the blurring effects of interstellar scattering with the resolution of an interferometer. In Figure 7.1 I plot the minimum size of resolvable structures on the image of Sgr $A^{*}$ using the interstellar scattering law of Bower et al. (2006). I also estimate (dashed line) the resolution of a radio interferometer at a given wavelength $\lambda$ by the expression

$$
\theta_{\text {res }}=k \lambda / d
$$

with $k=1$ and a diameter $d=10^{4} \mathrm{~km} \sim d_{\text {earth }}$, which is comparable to the baseline length between the JCMT on Hawaii and the South Pole Telescope. This yields 
$\theta_{\text {res }, \mu \text { as }}=21 \lambda_{\mathrm{mm}}$, where $\theta_{\text {res }, \mu \text { as }}$ is the resolution in $\mu$ arcsec and $\lambda_{\mathrm{mm}}$ is the observed wavelength in $\mathrm{mm}$. Dotted lines mark the angular diameters corresponding to length scales of $10 r_{g}$ and $1 r_{g}$ at the assumed distance of Sgr $\mathrm{A}^{*}$. As can be seen from this figure, at sub-mm wavelengths interstellar scattering becomes negligible and measurements are limited by the resolution. Therefore, a measurement of the diameter of the photon ring of Sgr $A^{*}$ will require VLBI observations at wavelengths $\lambda \lesssim 1 \mathrm{~mm}$.

In the following, I estimate the observed flux of the photon ring of Sgr A* assuming a Schwarzschild black hole and employing a model of a geometrically thin advection-dominated accretion flow (ADAF; Narayan \& Yi 1994, 1995; Narayan, Yi, \& Mahadevan 1995). ADAFs model the accreting gas around a supermassive black hole as a quasi-spherically symmetric plasma consisting of thermal electrons and ions at different temperatures (Narayan \& Yi 1995a, b) as well as of non-thermal electrons (Mahadevan 1999; Özel, Psaltis, \& Narayan 2000; Yuan, Quataert, \& Narayan 2003). Such an accretion flow is allowed to cool through comptonization and the emission of bremsstrahlung and synchrotron radiation, with the latter generating the predominant contribution to the observed spectrum of Sgr A* at radio and sub-mm wavelengths (Narayan et al. 1995).

For my estimate, I follow Broderick et al. (2009) and assume an ADAF model with a density of thermal electrons

$$
n_{e}(r)=n_{e 0}\left(\frac{r}{r_{g}}\right)^{-1.1}
$$

electron temperature

$$
T_{e}(r)=T_{e 0}\left(\frac{r}{r_{g}}\right)^{-0.84}
$$


and magnetic field

$$
\frac{B^{2}}{8 \pi}=\beta^{-1} n_{e} \frac{2 G M_{0} m_{p}}{12 r}
$$

where $\beta=10$. For the coefficients I use (Broderick et al. 2011)

$$
n_{e 0}=3 \times 10^{7} \mathrm{~cm}^{-3}
$$

and

$$
T_{e 0}=1.7 \times 10^{11} \mathrm{~K}
$$

These coefficients lead to predictions of the spectrum, polarization, and image size for Sgr A* that are in agreement with all current observations.

I assume that all the emission at mm-wavelengths is due to thermal synchrotron radiation (see Narayan et al. 1995). While there may be small contributions of synchrotron emission from non-thermal electrons (e.g., Mahadevan 1998; Özel et al. 2000; Yuan et al. 2003) or of other types of radiation at these wavelengths (such as jets; e.g., Falcke et al. 1993), this assumption affects my analysis only marginally. In practice, the measured total flux incorporates all such contributions.

Following Dolence et al. (2009), I write the synchrotron emissivity as

$$
j_{\nu} \simeq \frac{\sqrt{2} \pi e^{2} n_{e} \nu_{s}}{3 c K_{2}\left(1 / \theta_{e}\right)}\left(X^{1 / 2}+2^{11 / 12} X^{1 / 6}\right)^{2} \exp \left(-X^{1 / 3}\right),
$$

where

$$
\begin{gathered}
X \equiv \frac{\nu}{\nu_{s}}, \\
\nu_{s} \equiv \frac{2}{9}\left(\frac{e B}{2 \pi m_{e} c}\right) \theta_{e}^{2} \sin \alpha, \\
\theta_{e} \equiv \frac{k T}{m_{e} c^{2}}
\end{gathered}
$$


and $K_{2}$ is the modified Bessel function of the second kind of the second order. Here, $\alpha$ is the angle between the wave vector of the emitted photon and the magnetic field. For my estimate, I use the average $<\sin \alpha>=\pi / 4$.

I assume that optical paths follow at least one loop along the circular photon orbit of a Schwarzschild black hole located at radius $r=3 r_{g}$. The emitted intensity of radiation is then given by the expression

$$
I_{\mathrm{em}}=6 \pi r_{g} j_{\nu}
$$

The observed intensity is related to the emitted intensity by the third power of the redshift factor, which I take to be the gravitational redshift of a photon observed at infinity emitted from $r=3 r_{g}$ (i.e., I neglect for this simple estimate the high velocity of the flow, which will serve to increase the intensity at infinity). This yields:

$$
I_{\mathrm{obs}}=(1+z)^{-3} I_{\mathrm{em}},
$$

where

$$
1+z=\frac{1}{\sqrt{1-\frac{2}{3}}}=\sqrt{3}
$$

The photon ring has an approximate width of

$$
\Delta L \simeq 0.1 r_{g} \cong 0.51 \mu \operatorname{arcsec}
$$

in the image plane (Johannsen \& Psaltis 2010). Then, the subtended solid angle is given by the expression

$$
\Omega=\frac{L \times \Delta L}{D_{0}^{2}} \simeq \frac{\pi r_{g}^{2}}{D_{0}^{2}}
$$

This yields my estimate for the observed flux density of the photon ring:

$$
F_{\nu, \text { ring }}=I_{\mathrm{obs}} \times \Omega
$$




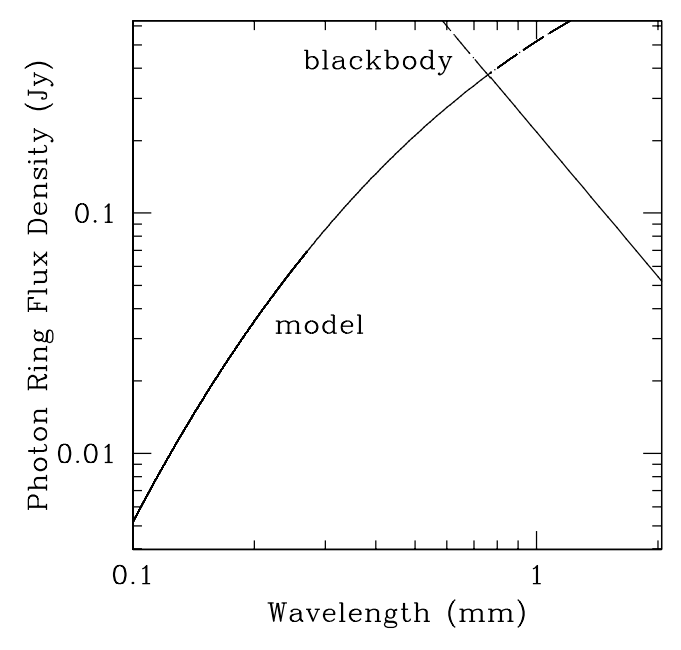

Figure 7.2 Estimate of the flux density of the photon ring of Sgr A* assuming emission from a geometrically thin ADAF around a Schwarzschild black hole (curve labeled "model"). At wavelengths $\lambda \lesssim 0.8 \mathrm{~mm}$, the modeled flux density is of the order of $0.2 \mathrm{Jy}$. I also plot (curve labeled "blackbody") a blackbody function at the same temperature and emission radius, which sets an upper flux density limit to the ring emission of the ADAF model at wavelengths $\lambda \gtrsim 0.8 \mathrm{~mm}$. Beyond this wavelength, the emission becomes optically thick. The solid line marks my estimate of the ring flux density in both wavelength ranges corresponding to the minimum of both functions.

In Figure 7.2, I plot the modeled ring flux density as a function of wavelength. I also plot a blackbody function evaluated at the same emission radius and temperature given by expression (7.7) as an upper flux density limit. The above estimate exceeds the blackbody flux density at wavelengths $\lambda \gtrsim 0.8 \mathrm{~mm}$, and, thus, at these longer wavelengths, the emission becomes self-absorbed. Therefore, I use the minimum of these two flux densities as an estimate of the ring flux density. At wavelengths $\lambda \lesssim 0.8 \mathrm{~mm}$, the modeled flux density of the photon ring of Sgr $\mathrm{A}^{*}$ is $\sim 0.2 \mathrm{Jy}$, about $1 / 15$ of the total source flux density. Since $\lambda \lesssim 0.8 \mathrm{~mm}$ is also in 

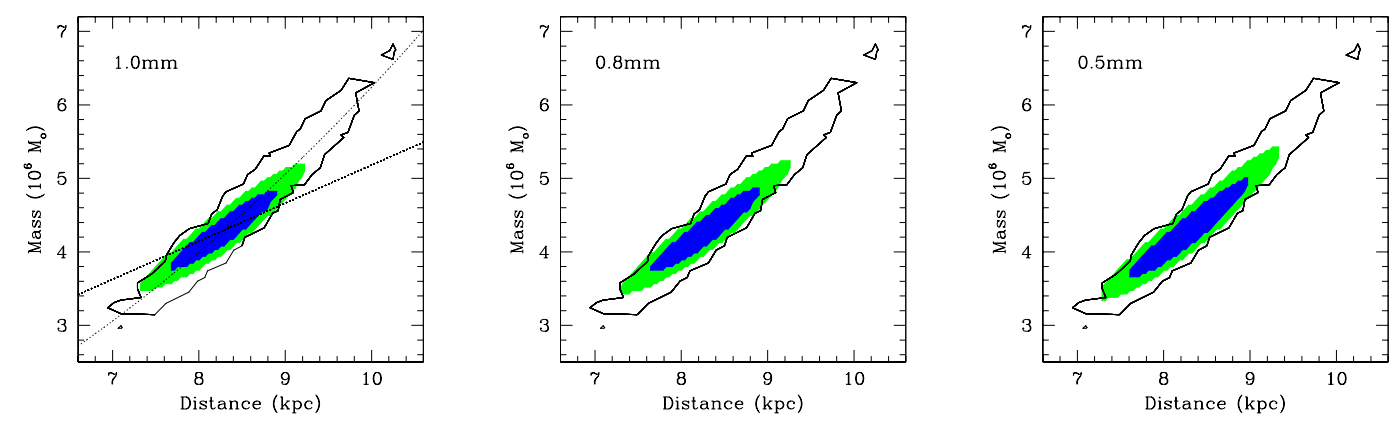

Figure $7.368 \%$ and $95 \%$ confidence contours of the mass and distance of Sgr A* for the combined distribution of stellar orbits and simulated VLBI measurements for a thermal noise-limited array at several wavelengths. Compared to (solid line) the $95 \%$ confidence contour of dynamical observations alone (Gillessen et al. 2009), the improvement of the mass and distance measurements is similar at all three wavelengths. For comparison, I also plot (left panel, dotted lines) the constant ratios $M / D$ and $M / D^{2}$ in order to illustrate the dependence of both individual methods on the correleation between mass and distance.

the regime where VLBI observations are resolution-limited (see Figure 7.1), this range of wavelengths is optimal for such measurements.

The ring diameter is determined solely by the mass via equation (7.1), and I can relate the black-hole mass and the angular diameter of the ring according to the expression

$$
\frac{M_{0}}{10^{6} M_{\odot}}=9.8 \times 10^{-3} \frac{\theta_{\text {ring }}}{\mu \operatorname{arcsec}} \frac{D_{0}}{\mathrm{kpc}} .
$$

Therefore, for VLBI imaging, the mass is proportional to the distance, and I can reduce the correlation between mass and distance in combination with dynamical measurements.

I now explore the prospect of combining dynamical measurements of Sgr A* with VLBI imaging observations of the photon ring. I analyze the best-case scenario of a thermal noise-limited VLBI array in order to assess whether such a 
measurement is worthwhile. Systematic limitations will degrade the VLBI measurement somewhat, as discussed below.

I employ a Bayesian analysis to estimate the probability distribution over the mass and distance of Sgr $\mathrm{A}^{*}$ from a measurement of the angular diameter of the photon ring in combination with the constraints obtained from stellar dynamics. I take the latter as my prior, $P_{\text {prior }}(M, D)$, by converting into a likelihood the $\chi^{2}$ distribution with $\nu=114$ degrees of freedom obtained from the existing data set of the ephemerides of several S-stars (Gillessen et al. 2009). I assume a Gaussian posterior likelihood of obtaining a particular measurement of the angular diameter as

$$
P_{\mathrm{VLBI}}(\text { data } \mid M, D)=\frac{1}{\sqrt{2 \pi \sigma^{2}}} \exp \left[-\frac{\left(\theta_{\text {ring }}-\theta_{0}\right)^{2}}{2 \sigma^{2}}\right],
$$

where $\theta_{\text {ring }}$ is given by equation (7.3) and $\theta_{0}=53 \mu$ arcsec. I then use Bayes' theorem to write the likelihood of a particular mass and distance of the black hole given the data as

$$
P(M, D \mid \text { data })=C P_{\mathrm{VLBI}}(\text { data } \mid M, D) P_{\text {prior }}(M, D)
$$

where $C$ is the appropriate constant that normalizes the likelihood.

The measurement uncertainty $\sigma$ is the key parameter of the likelihood $P_{\mathrm{VLBI}}$. In the following, I estimate the scaling of the measurement uncertainty with resolution. The spatial frequencies of interest are those beyond the first null of the Bessel function describing the Fourier transform of the ring structure in visibility space. For a given resolution and ring size, there are $N$ accessible half periods of the oscillation, where $N$ is given by

$$
N \equiv \frac{\theta_{0}}{\theta_{\text {res }}}-1=\frac{53}{21 \lambda_{\mathrm{mm}}}-1
$$


The typical amplitude of a Bessel function oscillation of the first few maxima is of order 0.3 of the peak. Therefore, the signal I seek to measure has an amplitude of

$$
A \equiv 0.3 \times \frac{F_{\nu, \text { ring }}}{F_{\nu, \text { flow }}} \times N=0.3 \times \frac{0.2}{3} \times N=0.02 N,
$$

where $F_{\nu \text {,flow }} \simeq 3 \mathrm{Jy}$ is the observed flux density of Sgr $\mathrm{A}^{*}$ near $1 \mathrm{~mm}$ (see, e.g., Broderick et al. 2009).

I estimate the expected signal to noise ratio on this measurement by scaling it from the current VLBI measurements. Fish et al. (2011) measure the size of Sgr A* with a signal to noise ratio of $\sim 40$. Near-future VLBI arrays will incorporate 5 or 6 stations. One of them, ALMA, will have the sensitivity of 50 of the current stations resulting in an overall increase in array sensitivity of a factor of up to roughly 9.3. In addition, the scheduled increase of recording bandwidth will increase the sensitivity by a factor of $\sqrt{8}$. To account for the variation in system temperature for typical observing conditions and receiver performance I introduce a degradation in SNR proportional to the observing wavelength squared, normalized to the $1.3 \mathrm{~mm}$ performance in Fish et al. (2011). The total signal to noise ratio of this measurement will then be

$$
\begin{aligned}
\mathrm{SNR}= & 9.3 \times \sqrt{8} \times 40 \times A \times\left(\frac{\lambda}{1.3 \mathrm{~mm}}\right)^{2} \\
& \simeq 12 \times \lambda_{\mathrm{mm}}^{2}\left(\frac{53}{21 \lambda_{\mathrm{mm}}}-1\right)
\end{aligned}
$$

with the width of the distribution given by the expression

$$
\sigma=\frac{\theta_{0}}{\mathrm{SNR}} \simeq 4.3 \times \lambda_{\mathrm{mm}}^{-2}\left(\frac{53}{21 \lambda_{\mathrm{mm}}}-1\right)^{-1} \mu \operatorname{arcsec} .
$$

In Figure 7.3, I plot confidence contours of the joint probability distribution for the combination of future thermal noise-limited VLBI and current astrometric 
observations at three different wavelengths. The solid line marks the $95 \%$ confidence contour determined by the stellar ephemerides (Gillessen et al. 2009). A VLBI measurement at a wavelength of $1 \mathrm{~mm}$ significantly improves the result from stellar orbits alone. At smaller wavelengths, the constraints on the mass and distance of Sgr $\mathrm{A}^{*}$ are similar. In the rightmost panel of Figure 3, I have extrapolated the distribution width $\sigma$ given by expression (7.27) to a nominal wavelength of $\lambda=0.5 \mathrm{~mm}$. Measurements at such short wavelengths will be limited by weather conditions and may have to rely on a smaller array with fewer telescopes.

Real observations will face more stringent limitations than those imposed by the interferometer thermal noise due to the complications of astrophysics and measurement systematics. The chief astrophysical limitation is the separation of the ring emission from the source structure in the uv-plane. In my estimate, I have used the location of the nulls as a benchmark for the uncertainties I expect from the VLBI measurement. In practice, however, the full visibility function has to be analyzed with a pattern matching technique that identifies the structure of the ring. Such a technique has to extract the ring from a uv-plane that is only partially sampled by a given set of baselines.

The physics of the accretion flow will also complicate things, as the structure of Sgr A* may vary over the course of an observation. However, because the ring structure is persistent and only weakly altered by rapid changes in the accretion flow I expect that temporal averaging of the visibilities across multiple observing epochs will diminish the importance of such changes.

The VLBI measurement itself must surmount systematic limitations to make the moderate dynamic range measurements proposed here. Chief among these is 
the difficulty of calibrating the noise level at individual stations, which imposed a 5\% uncertainty in Fish et al. (2011) with the three-station array. In observations with the larger array considered here, there will be many more internal crosschecks available to improve the relative calibration of stations (the absolute calibration is not important). In particular, the use of three phased interferometers (Mauna Kea, CARMA, ALMA) that simultaneously record conventional interferometric data will permit scan-by-scan cross calibration of the amplitude scale of the array.

Furthermore, the larger arrays will be able to make use of closure phases and closure amplitudes that are immune to calibration errors as part of the ring detection, although I have ignored such procedures here because of the difficulty of simply parameterizing the improvement they can permit. Other effects, such as the coherence of the reference systems between stations (reported as $<5 \%$ in Fish et al. 2011) can be more carefully measured and corrected to prevent them from imposing fundamental limitations to the ring detection. 


\subsection{Other Sources}

Besides Sgr A*, there exist other nearby supermassive black holes, for which a combination of dynamical measurements and VLBI observations could be feasible. Since these supermassive black holes are located in host galaxies other than the Milky Way, observations are much less affected by interstellar scattering. As an example, Broderick \& Loeb (2009) and Takahashi \& Mineshige (2011) analyzed the prospects of imaging the shadow of M87 with VLBI observations at several different wavelengths.

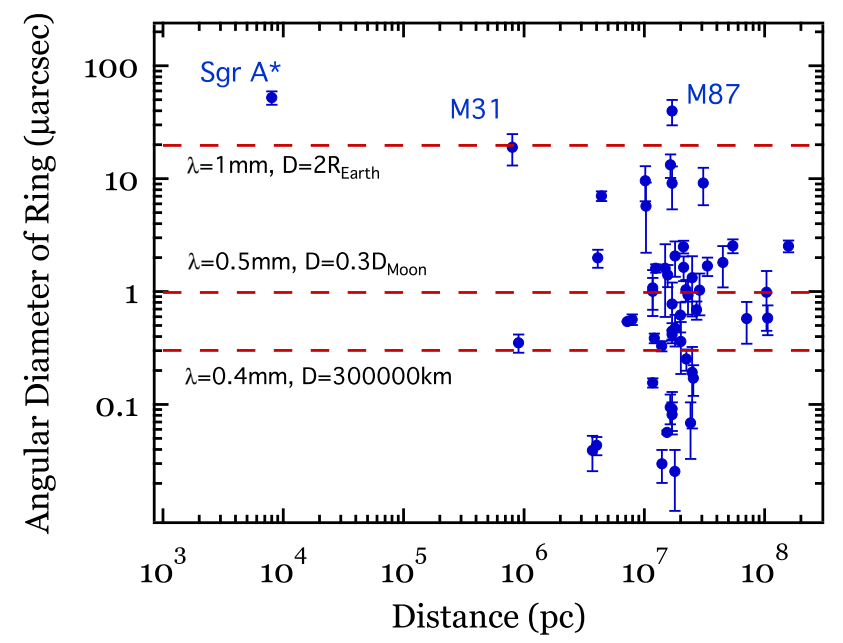

Figure 7.4 Angular diameters and distances of several supermassive black holes. Sgr A* has the largest angular diameter, closely followed by M87 due to its high mass, making these sources ideal targets for VLBI imaging. Data taken from Gültekin et al. (2009).

In Figure 7.4, I plot the angular diameter of the photon rings against the distances of a collection of nearby supermassive black holes. Sgr $A^{*}$ is closest to us and has the largest angular diameter, closely followed by M87 and M31 due to their large black hole masses. The top dashed line indicates the resolution of a 
telescope array with a baseline equal to the diameter of the Earth (from equation (7.5)) at a wavelength of $1 \mathrm{~mm}$. For comparison, I also show the resolution of a future space telescope located at 30\% the distance to the moon (comparable to the orbit of Chandra) at a wavelength of $0.5 \mathrm{~mm}$ corresponding to an angular diameter of about $1 \mu$ arcsec as well as of the proposed Millimetron mission (Wild et al. 2009) at a distance of $3 \times 10^{5} \mathrm{~km}$ and a wavelength of $\lambda=0.4 \mathrm{~mm}$.

In order to be able to resolve the photon ring with VLBI, the key question is whether the accretion flow of the target supermassive black hole is optically thin. In some cases, the spectra of these sources peak at wavelengths near $\lambda_{\max } \sim 1 \mathrm{~mm}$, similar to the spectrum of Sgr $\mathrm{A}^{*}$, suggesting that the emission comes from an ADAF (Di Matteo et al. 2000; Doi et al. 2005). Naively, I would expect an approximately linear scaling of the electron density of an ADAF with the ratio $M / \dot{M}$ of the black hole, where $\dot{M}$ is its mass accretion rate. The details of such a relation depend on a variety of factors, such as the temperature profile, the emissivity, and the radiative efficiency. However, most of the nearby supermassive black holes have very low radiative efficiencies (Ho 2009). Therefore, it is plausible that the accretion flows of these nearby supermassive black holes become optically thin at wavelengths that are comparable to $1 \mathrm{~mm}$ making them accessible to VLBI observations. Such observations are best carried out at wavelengths near the flux peak, where the accretion flow is becoming optically thin. At wavelengths $\lambda \ll \lambda_{\max }$, the emitted flux is likely to be too low to be detected with a VLBI array, while at wavelengths $\lambda \gg \lambda_{\max }$, the accretion flow is optically thick.

In Table 7.1, I summarize the angular diameters, distances, masses, radio luminosities $L_{R}$, flux densities $S_{\nu}$ near $1 \mathrm{~mm}$, ratios of the bolometric luminosity to the Eddington luminosity $L_{\mathrm{bol}} / L_{\mathrm{Edd}}$, and peak wavelengths $\lambda_{\max }$ for super- 
Table 7.1. Sources for VLBI Observations

\begin{tabular}{|c|c|c|c|c|c|c|c|c|}
\hline Source & $\begin{array}{c}\theta_{\text {ring }} \\
(\mu \operatorname{arcsec})\end{array}$ & $\begin{array}{c}\text { Distance }^{\mathrm{a}} \\
(\mathrm{Mpc})\end{array}$ & $\begin{array}{c}\log \left(M_{B H}\right)^{\mathrm{a}} \\
\left(M_{\odot}\right)\end{array}$ & $\begin{array}{c}\log \left(L_{R}\right)^{\mathrm{a}} \\
(\mathrm{erg} / \mathrm{s})\end{array}$ & $\begin{array}{l}S_{\nu} \\
(\mathrm{Jy})\end{array}$ & Ref & $\log \left(L_{\mathrm{bol}} / L_{\mathrm{Edd}}\right)^{\mathrm{b}}$ & $\begin{array}{l}\lambda_{\max } \\
(\mathrm{mm})\end{array}$ \\
\hline Sgr A* & 53 & 0.008 & $6.61 \pm 0.064$ & 32.48 & 2.4 & 1 & $\ldots$ & $\sim 1^{\mathrm{c}}$ \\
\hline NGC 4486 (M87) & 22 & 17.0 & $9.56 \pm 0.126$ & 39.83 & 0.897 & 2 & -5.15 & $\sim 1^{\mathrm{d}}$ \\
\hline NGC 0224 (M31) & 19 & 0.8 & $8.17 \pm 0.161$ & 32.14 & $\approx 3 \times 10^{-5}$ & 3 & -8.90 & \\
\hline NGC 4649 (M60) & 13 & 16.5 & $9.33 \pm 0.117$ & 37.45 & $<0.004$ & 4 & -7.84 & $\sim 3^{\mathrm{d}}$ \\
\hline NGC 3115 & 9.6 & 10.2 & $8.98 \pm 0.182$ & $\ldots$ & $\ldots$ & & -7.03 & \\
\hline IC 1459 & 9.2 & 30.9 & $9.44 \pm 0.196$ & 39.76 & 0.264 & 5 & $\cdots$ & \\
\hline NGC 4374 (M84) & 9.1 & 17.0 & $9.18 \pm 0.231$ & 38.77 & 0.129 & 6 & -6.29 & \\
\hline NGC 5128 (Cen A) & 7.0 & 4.4 & $8.48 \pm 0.044$ & 39.85 & 6.9 & 7 & $\ldots$ & \\
\hline NGC 4594 (M104) & 5.7 & 10.3 & $8.76 \pm 0.413$ & 37.89 & 0.25 & 8 & -4.68 & \\
\hline IC 4296 & 2.5 & 54.4 & $9.13 \pm 0.065$ & 38.59 & 0.155 & 9 & $\ldots$ & \\
\hline NGC 1399 & 2.5 & 21.1 & $8.71 \pm 0.060$ & $\ldots$ & $\approx 0.04$ & 3 & $\ldots$ & $\sim 3^{\mathrm{d}}$ \\
\hline NGC 4342 & 2.1 & 18.0 & $8.56 \pm 0.185$ & $\ldots$ & $\ldots$ & & $\ldots$ & \\
\hline NGC 3031 (M81) & 2.0 & 4.1 & $7.90 \pm 0.087$ & 36.97 & 0.1812 & 10 & -5.29 & $\sim 10^{\mathrm{e}}$ \\
\hline NGC 4261 & 1.7 & 33.4 & $8.74 \pm 0.090$ & 39.32 & 0.059 & 11 & -5.21 & \\
\hline NGC 3585 & 1.6 & 21.2 & $8.53 \pm 0.122$ & $\ldots$ & $\ldots$ & & $\ldots$ & \\
\hline NGC 3998 & 1.6 & 14.9 & $8.37 \pm 0.431$ & 38.03 & $<0.007$ & 8 & -4.43 & \\
\hline NGC 4697 & 1.6 & 12.4 & $8.29 \pm 0.038$ & $\ldots$ & $<0.007$ & 8 & $\ldots$ & \\
\hline NGC 4026 & 1.4 & 15.6 & $8.33 \pm 0.109$ & $\ldots$ & $\ldots$ & & $\ldots$ & \\
\hline NGC 3379 (M105) & 1.1 & 11.7 & $8.09 \pm 0.250$ & 35.81 & $\ldots$ & & -7.57 & \\
\hline NGC 3245 & 1.0 & 22.1 & $8.35 \pm 0.106$ & 36.98 & $\ldots$ & & -5.83 & \\
\hline NGC 5845 & 1.0 & 28.7 & $8.46 \pm 0.223$ & $\ldots$ & $\ldots$ & & $\ldots$ & \\
\hline NGC 3377 & 1.0 & 11.7 & $8.06 \pm 0.163$ & $\ldots$ & $\ldots$ & & -6.16 & \\
\hline
\end{tabular}

Note. - (a) Gültekin et al. (2009); (b) L. Ho, priv. commun.; (c) Broderick et al. (2009); (d) Di Matteo et al. (2000); (e) Doi et al. (2005); For $S_{\nu}$ : (1) VLBI: $1.3 \mathrm{~mm}$ (Doeleman et al. 2008); (2) VLBI: $86 \mathrm{GHz}$ (Lee et al. 2008); (3) Power law fit of mm-data from NASA Extragalactic Database, evaluated at $1 \mathrm{~mm}$; (4) Submillimetre CommonUser Bolometer Array (SCUBA): 2 mm (Di Matteo et al. 1999); (5) Australia Telescope Compact Array (ATCA): $95 \mathrm{GHz}$ (Sadler et al. 2008); (6) NObeyama Bolometer Array (NOBA): $2 \mathrm{~mm}$ (Leeuw et al. 2004); (7) Swedish-ESO 15m Submillimeter Telescope (SEST): $2 \mathrm{~mm}$ (Israel et al. 2008); (8) SCUBA: $0.85 \mathrm{~mm}$ (Bendo et al. 2006); (9) Very Long Baseline Array (VLBA): 8.4 GHz (Pellegrini et al. 2003); (10) Plateau de Bure Interferometer (PdBI): 241.4 GHz (Schödel et al. 2007); (11) VLBI: 86 GHz (Middelberg et al. 2005). 
massive black holes, whose photon rings have an angular diameter of at least $1 \mu \operatorname{arcsec}$.

In addition to Sgr $\mathrm{A}^{*}$, the black holes in the centers of M87, M31, and M60 are good potential targets for VLBI observations, because of the large angular diameters of their respective photon rings and, in the case of M87 and M60, the measured peak in the synchrotron part of their spectra near $1 \mathrm{~mm}$. M87 has a high measured flux density at $86 \mathrm{GHz}$ (Lee et al. 2008) and should be readily observable at wavelengths close to $1 \mathrm{~mm}$. In the case of M60, however, Di Matteo et al. (1999) report an upper limit on the flux density of $4 \mathrm{mJy}$. No similar flux density measurement of M31 has been reported to date. I estimate the flux density at $1 \mathrm{~mm}$ of M31 from a simple power law fit of mm-data from the NASA Extragalactic Database. This flux density is relatively small, and M31 as well as M60 may be too faint to be observable with VLBI. Other sources, such as Centaurus $\mathrm{A}$, are luminous enough to be detectable at wavelengths near $1 \mathrm{~mm}$. With increasing VLBI resolution, even their photon rings may become observable.

In the following, I assess the improvement on the mass measurements of the two supermassive black holes (Sgr $\mathrm{A}^{*}$ and M87) whose photon rings have the largest angular diameters. I assume fixed distances of $D_{0}=8.3 \mathrm{kpc}$ (Gillessen et al. 2009) and $D=17 \mathrm{Mpc}$ (Gültekin et al. 2009) for Sgr A* and M87, respectively. For Sgr A*, I estimate an error of the combined mass measurement from the existing stellar ephemerides and my simulated VLBI data of only $\sim 5 \%$ (see Figure 7.3).

For M87, I estimate the smallest relative error that thermal noise-limited VLBI imaging observations of the ring can achieve from the signal to noise ratio for observations of Sgr $\mathrm{A}^{*}$ given by equation (7.26), which I scale with the angular 
diameter of the black hole to obtain the expression

$$
\frac{\delta M}{M}=\frac{\delta \theta_{\text {ring }}}{\theta_{\text {ring }}} \simeq \frac{1}{12} \lambda_{\mathrm{mm}}^{-2}\left(\frac{\theta_{\text {ring }}}{21 \lambda_{\mathrm{mm}}}-1\right)^{-1} .
$$

Note, however, that M87 has a much larger mass and that the dynamical timescales of its accretion flow is much longer. Therefore, VLBI imaging observations of its photon ring will be much less affected by the variability of the accretion flow as in the case of Sgr A*.

In Table 7.2, I compare the relative errors of the mass measurements of Sgr $\mathrm{A}^{*}$ and M87 (Gillessen et al. 2009; Gültekin et al. 2009) with my estimate of the error of VLBI observations of the respective photon rings at several different wavelengths. In the case of Sgr $\mathrm{A}^{*}$, imaging its photon ring improves the error by a factor of about two. In the case of M87, imaging the photon ring at a wavelength of $0.5 \mathrm{~mm}$ would lead to a result that is similar to the current mass measurement.

\begin{tabular}{lcccc}
\multicolumn{5}{c}{ Relative Errors of Mass Measurements } \\
\hline \hline Source & $\left.\frac{\delta M}{M}\right|_{\mathrm{dyn}}$ & $\left.\frac{\delta M}{M}\right|_{\mathrm{VLBI}}$ & $\left.\frac{\delta M}{M}\right|_{\mathrm{VLBI}}$ & $\left.\frac{\delta M}{M}\right|_{\mathrm{VLBI}}$ \\
$(1.0 \mathrm{~mm})$ & $(0.8 \mathrm{~mm})$ & $(0.5 \mathrm{~mm})$ \\
\hline Sgr A $^{*}$ & 0.09 & 0.05 & 0.05 & 0.05 \\
M87 & 0.29 & 1.75 & 0.42 & 0.30 \\
\hline
\end{tabular}

Table 7.2 Relative errors of mass measurements from dynamical observations and VLBI simulations.

As I pointed out in the previous section for the case of $\operatorname{Sgr} \mathrm{A}^{*}$, these errors require further refinement by in-depth imaging simulations. In addition, the morphology of (sub-)mm VLBI emission can be complicated by the presence of a jet (for M87, see Broderick \& Loeb 2009; Dexter et al. 2011). 
As in the case of Sgr $\mathrm{A}^{*}$, the combination of the results from both the dynamical and VLBI imaging observations of M87 would further reduce the error in the masses. The relative errors of mass measurements of both techniques likewise depend on the error in the measured distances to these sources. These errors, in turn, depend on uncertainties in the Hubble constant, peculiar motions of the gas in host galaxies, as well as assumptions on the proper motion of the Milky Way (see, e.g., Hodge 1981; Jacoby et al. 1992). The details of these effects on the relative errors of the mass are beyond the scope of my analysis. 


\subsection{Discussion}

In this chapter, I investigated the prospects of measuring the mass and distance of Sgr A* as well as the masses of several other nearby supermassive black holes with a combination of dynamical observations and VLBI imaging of the respective photon rings of these sources. In order to resolve the photon ring of a black hole, its accretion flow must be optically thin. I argued that the wavelengths at which the accretion flows of these sources become optically thin should be roughly comparable to the location of the peak in the synchrotron emission of Sgr $\mathrm{A}^{*}$ and identified several supermassive black holes as optimal targets.

I explored the prospects of imaging the photon ring of Sgr $\mathrm{A}^{*}$ as well as of other nearby supermassive black holes with near-future VLBI arrays. I estimated the signal to noise ratio with which such arrays can image the photon ring in the best-case scenario if the VLBI observations are limited by thermal noise. Based on my estimate, I simulated confidence contours of a mass measurement of Sgr A* using existing data of stellar ephemerides. I showed that the combination of both techniques can indeed reduce the correlation between mass and distance significantly resulting in relative errors of the mass and distance of only a few percent. I also identified several sources of uncertainty that have to be taken into account for an actual detection of the photon ring of Sgr $\mathrm{A}^{*}$.

The uncertainties of measurements based on stellar orbits will be further reduced by the continued monitoring and by the expected improvement in astrometry possible with the second generation instrument GRAVITY for the Very Large Telescope Interferometer (Eisenhauer et al. 2011). Further improvements of the VLBI sensitivity will be achieved by the Event Horizon Telescope, a planned global array of (sub-)mm telescopes (Doeleman et al. 2009a, b; Fish et al. 2009). 
I estimated the improvement of the mass measurement of M87 using VLBI techniques. Such observations are promising at wavelengths near $0.5 \mathrm{~mm}$ because of the large size of its photon ring. For M31 and M60, the supermassive black holes with the largest photon rings besides Sgr $\mathrm{A}^{*}$ and M87, the flux densities may be too low to be detectable with VLBI. As the resolution of VLBI arrays increases, additional sources will become observable.

Angular resolution of $\sim 1 \mu$ arcsec requires longer baselines and/or shorter observing wavelengths. However, the atmosphere precludes regular VLBI observations at wavelengths shorter than $\sim 0.3 \mathrm{~mm}$ at even the best sites.

A measurement of the photon-ring diameter is likely to yield useful results only if the observations extend well beyond the first null in the ring's visibility function. Even at a wavelength of $\lambda \simeq 0.4 \mathrm{~mm}$, space-VLBI observations will be required to reach this point for all except the first four entries in Table 1.

VLBI between Earthbound antennas and a satellite has been achieved at $6 \mathrm{~cm}$ wavelength using the Japanese HALCA satellite (Hirabayashi et al. 1998). The recently launched RadioAstron (Kardashev 2009) will extend such observations to $1.2 \mathrm{~cm}$ wavelength and baselines as large as $4 \times 10^{5} \mathrm{~km}$, for a resolution of $<10 \mu$ arcsec.

A future Explorer-class space mission designed to observe at $1 \mathrm{~mm}$ wavelength or shorter, where source opacity and scattering effects will be far less important, could provide the angular resolution needed to study a far larger sample of sources. Such a capability may be provided by the Russian-European Millimetron mission, which plans to deploy a $12 \mathrm{~m}$ antenna with VLBI capabilities to $0.4 \mathrm{~mm}$ and maximum baseline $>3 \times 10^{5} \mathrm{~km}$ (Wild et al. 2009). 


\section{CHAPTER 8}

QuASI-PERIODIC VARIABILITY

In this chapter, I derive expressions for the Keplerian and epicyclic frequencies of circular equatorial motion in the quasi-Kerr spacetime. I discuss the properties of these frequencies and analyze their distinct dependencies on the mass, spin, and quadrupole moment of the black-hole spacetime. In particular, I demonstrate how this formalism can be applied in order to test the no-hair theorem with the quasi-periodic variability observed from galactic black holes, AGN, and Sgr A*. The contents of this chapter were published in Johannsen \& Psaltis (2011b). 


\subsection{Dynamical Frequencies in the Quasi-Kerr Spacetime}

In this section, I systematically derive expressions for the Keplerian frequency $\Omega_{\phi}$ of a particle on a circular equatorial orbit around a quasi-Kerr black hole as well as for the frequencies of small oscillations $\kappa_{\mathrm{r}}$ and $\Omega_{\theta}$ in the two directions perpendicular to such an orbit. For an alternative computation of the frequencies $\Omega_{\phi}$ and $\kappa_{\mathrm{r}}$, see Glampedakis \& Babak (2006).

Since the quasi-Kerr metric given by expression (2.13) is stationary and axisymmetric, particle trajectories in this metric are characterized by three integrals of motion. For a particle with 4-momentum

$$
p^{\alpha}=\mu \frac{d x^{\alpha}}{d \tau},
$$

these are its rest mass $\mu$ (which I will set equal to unity from here on), energy $E=-p_{\mathrm{t}}$, and angular momentum about the $z$-axis $L_{\mathrm{z}}=p_{\phi}$.

I use the conservation of energy and axial angular momentum to express the momentum components $p^{t}$ and $p^{\phi}$ of a particle in the form

$$
\begin{gathered}
p^{\mathrm{t}}=-\frac{g_{\phi \phi} E+g_{\mathrm{t} \phi} L_{\mathrm{z}}}{g_{\mathrm{t} \mathrm{t}} g_{\phi \phi}-g_{\mathrm{t} \phi}^{2}}, \\
p^{\phi}=\frac{g_{\mathrm{t} \phi} E+g_{\mathrm{tt}} L_{\mathrm{z}}}{g_{\mathrm{tt}} g_{\phi \phi}-g_{\mathrm{t} \phi}^{2}} .
\end{gathered}
$$

I then bring the conservation equation for the particle's rest mass,

$$
g_{\mu \nu} p^{\mu} p^{\nu}=-1,
$$

into the form

$$
\frac{1}{2}\left(g_{\mathrm{rr}} \dot{r}^{2}+g_{\theta \theta} \dot{\theta}^{2}\right)=\frac{1}{2}\left[-g_{\mathrm{tt}}\left(p^{\mathrm{t}}\right)^{2}-2 g_{\mathrm{t} \phi} p^{\mathrm{t}} p^{\phi}-g_{\phi \phi}\left(p^{\phi}\right)^{2}-1\right] \equiv V_{\mathrm{eff}}
$$


with $V_{\text {eff }}$ playing the role of an effective potential for the particle motion in the coordinates $r$ and $\theta$.

The radial motion in the equatorial plane can be analyzed in terms of the equation

$$
\frac{1}{2}\left(\frac{d r}{d t}\right)^{2}=\frac{V_{\mathrm{eff}}}{g_{\mathrm{rr}}\left(p^{\mathrm{t}}\right)^{2}} \equiv V_{\mathrm{eff}}^{\mathrm{r}},
$$

while the $\theta$-motion (for constant radii $r$ ) is governed by the equation

$$
\frac{1}{2}\left(\frac{d \theta}{d t}\right)^{2}=\frac{V_{\text {eff }}}{g_{\theta \theta}\left(p^{t}\right)^{2}} \equiv V_{\text {eff }}^{\theta} .
$$

In Chapter 2, I solved the equations

$$
V_{\text {eff }}^{\mathrm{r}}=0
$$

and

$$
\frac{d V_{\mathrm{eff}}^{\mathrm{r}}}{d r}=0,
$$

which characterize circular equatorial orbits, and obtained the energy

$$
\begin{aligned}
E= & \frac{r^{3 / 2}-2 M r^{1 / 2} \pm a M^{1 / 2}}{r^{3 / 4} \sqrt{r^{3 / 2}-3 M r^{1 / 2} \pm 2 a M^{1 / 2}}}-\frac{5 \epsilon}{32 M^{2} r^{3 / 2}(r-3 M)^{3 / 2}} \\
& \times\left[2 M\left(6 M^{4}+14 M^{3} r-41 M^{2} r^{2}+27 M r^{3}-6 r^{4}\right)\right. \\
& \left.+r^{2}\left(6 r^{3}-33 M r^{2}+66 M^{2} r-48 M^{3}\right) \ln \left(\frac{r}{r-2 M}\right)\right]
\end{aligned}
$$

and axial angular momentum

$$
\begin{aligned}
L_{z}= & \pm \frac{M^{1 / 2}\left(r^{2} \mp 2 a M^{1 / 2} r^{1 / 2}+a^{2}\right)}{r^{3 / 4} \sqrt{r^{3 / 2}-3 M r^{1 / 2} \pm 2 a M^{1 / 2}}} \mp \frac{5 \epsilon}{32 M^{5 / 2}(r-3 M)^{3 / 2}} \\
& \times\left[2 M\left(6 M^{4}-7 M^{3} r-16 M^{2} r^{2}+12 M r^{3}-3 r^{4}\right)\right. \\
& \left.+3 r\left(r^{4}-5 M r^{3}+9 M^{2} r^{2}-2 M^{3} r-6 M^{4}\right) \ln \left(\frac{r}{r-2 M}\right)\right] .
\end{aligned}
$$


These expressions are expansions to linear order in the parameter $\epsilon$, where I have neglected terms of the order $\epsilon a$. An expansion of this type is implicitly understood for all expressions throughout the chapter in accordance with the form of the metric specified by expression (2.13). Note, however, that I do not expand in the spin parameter $a$ so that my results are correct for arbitrary values of the spin in the special case $\epsilon=0$.

\subsubsection{The Keplerian Frequency $\Omega_{\phi}$}

For a particle moving on a circular orbit in the equatorial plane, the time and azimuthal components of the 4-momentum take the form (Glampedakis \& Babak 2006)

$$
\begin{aligned}
p^{\mathrm{t}} & =\frac{1}{\Delta}\left[E\left(r^{2}+a^{2}\right)+\frac{2 M a}{r}\left(a E-L_{\mathrm{z}}\right)\right]-\epsilon\left(1-\frac{2 M}{r}\right)^{-1} f_{3}(r) E, \\
p^{\phi} & =\frac{1}{\Delta}\left[\frac{2 M}{r}\left(a E-L_{\mathrm{z}}\right)+L_{\mathrm{z}}\right]-\epsilon \frac{h_{3}(r)}{r^{2}} L_{\mathrm{z}}
\end{aligned}
$$

where

$$
\begin{aligned}
f_{3}(r)= & -\frac{5(r-M)}{8 M r(r-2 M)}\left(2 M^{2}+6 M r-3 r^{2}\right) \\
& -\frac{15 r(r-2 M)}{16 M^{2}} \ln \left(\frac{r}{r-2 M}\right), \\
h_{3}(r)= & \frac{5}{8 M r}\left(2 M^{2}-3 M r-3 r^{2}\right)+\frac{15}{16 M^{2}}\left(r^{2}-2 M^{2}\right) \ln \left(\frac{r}{r-2 M}\right) .
\end{aligned}
$$

From these expressions, I obtain the Keplerian frequency

$$
\begin{aligned}
\Omega_{\phi} \equiv & \frac{d \phi}{d t}=\frac{p^{\phi}}{p^{\mathrm{t}}}= \pm \frac{\sqrt{M}}{r^{3 / 2} \pm a \sqrt{M}} \mp \frac{5 \epsilon}{32 M^{5 / 2}(r-2 M) r^{5 / 2}} \\
& \times\left[2 M\left(-6 M^{4}+8 M^{3} r+2 M^{2} r^{2}+3 M r^{3}-3 r^{4}\right)\right. \\
& \left.+3 r(r-2 M)\left(r^{3}-2 M^{3}\right) \ln \left(\frac{r}{r-2 M}\right)\right] .
\end{aligned}
$$




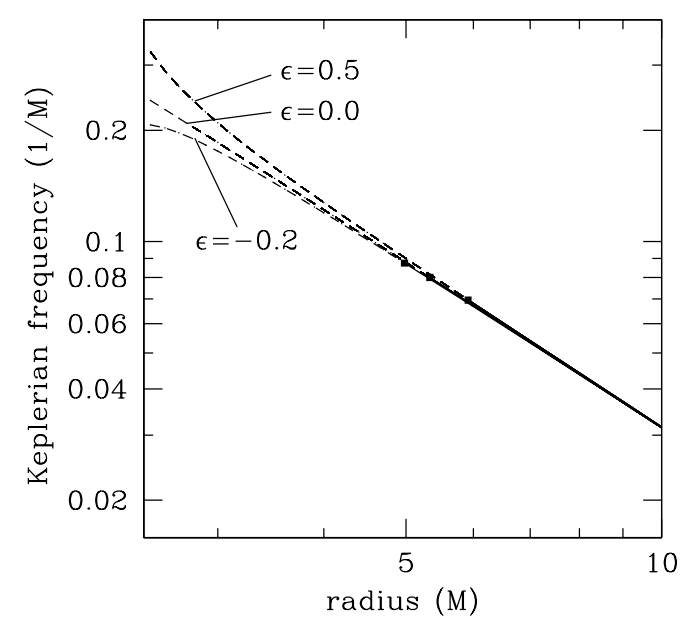

Figure 8.1 Keplerian frequency $\Omega_{\phi}$ of a particle on a circular equatorial orbit around a quasi-Kerr black hole with a spin of $a=0.2 M$ and for several values of the parameter $\epsilon$. The Keplerian frequency increases for decreasing values of the radius and for increasing values of the parameter $\epsilon$. The dots mark the location of the ISCO, and the dashed lines correspond to unstable orbits.

In Figure 8.1 I plot the Keplerian frequency as a function of radius for several values of the parameter $\epsilon$ and a spin of $a=0.2 M$. The frequency increases at smaller radii. The dependence on the quadrupolar parameter $\epsilon$ is weak and only manifests at radii comparable to a few $M$. Increasing the value of the parameter $\epsilon$ leads to larger Keplerian frequencies.

\subsubsection{The Radial and Vertical Epicyclic Frequencies}

In order to calculate the radial and vertical epicyclic frequencies, I introduce small perturbations $\delta r$ and $\delta \theta$ around a circular equatorial orbit located at radius $r_{0}$. I take the (coordinate) time derivative of equations (8.6) and (8.7) and obtain

$$
\frac{d^{2}(\delta r)}{d t^{2}}=\frac{d^{2} V_{\mathrm{eff}}^{\mathrm{r}}}{d r^{2}} \delta r,
$$




$$
\frac{d^{2}(\delta \theta)}{d t^{2}}=\frac{d^{2} V_{\text {eff }}^{\theta}}{d \theta^{2}} \delta \theta
$$

using the effective potentials $V_{\text {eff }}^{\mathrm{r}}$ and $V_{\text {eff }}^{\theta}$ defined in equations (8.6) and (8.7). From these expressions, I derive the frequencies of small oscillations in the $r$ and $\theta$ directions around a circular equatorial orbit as

$$
\begin{aligned}
\kappa_{\mathrm{r}}^{2} & =-\frac{d^{2} V_{\mathrm{eff}}^{\mathrm{r}}}{d r^{2}}, \\
\Omega_{\theta}^{2} & =-\frac{d^{2} V_{\mathrm{eff}}^{\theta}}{d \theta^{2}} .
\end{aligned}
$$

Here the second derivatives of the effective potentials are evaluated at the energy and axial angular momentum given by expressions (8.10) and (8.11), and for $\theta=\pi / 2$. The result is:

$$
\begin{aligned}
\kappa_{\mathrm{r}}^{2}= & \frac{M\left(r^{2}-6 M r \pm 8 a \sqrt{M} \sqrt{r}-3 a^{2}\right)}{r^{2}\left(r^{3 / 2} \pm a \sqrt{M}\right)^{2}}-\frac{5 \epsilon}{16 M^{2} r^{5}(r-2 M)} \\
& \times\left[2 M\left(48 M^{5}+30 M^{4} r+26 M^{3} r^{2}-127 M^{2} r^{3}+75 M r^{4}-12 r^{5}\right)\right. \\
& \left.+3 r^{2}(r-2 M)^{2}\left(4 r^{2}-13 M r-2 M^{2}\right) \ln \left(\frac{r}{r-2 M}\right)\right]
\end{aligned}
$$

and

$$
\begin{aligned}
\Omega_{\theta}^{2}= & \frac{M\left(r^{2} \mp 4 a \sqrt{M} \sqrt{r}+3 a^{2}\right)}{r^{2}\left(r^{3 / 2} \pm a \sqrt{M}\right)^{2}}+\frac{5 \epsilon}{16 M^{2} r^{4}(r-2 M)} \\
& \times\left[2 M\left(6 M^{4}+34 M^{3} r-59 M^{2} r^{2}+33 M r^{3}-6 r^{4}\right)\right. \\
& \left.+3 r(2 r-M)(r-2 M)^{3} \ln \left(\frac{r}{r-2 M}\right)\right] .
\end{aligned}
$$

To convert these expressions to cgs units, one needs to multiply by $\left(c^{3} / G\right)^{2}$, where $c$ and $G$ are the speed of light and the gravitational constant, respectively. In the special case where $\epsilon=0$, these expressions coincide with the ones in Okazaki et al. (1987) and Kato (1990). Similar calculations have also been performed by Shibata \& Sasaki (1998), Berti \& Stergioulas (2004), Gair et al. (2008), and SanabriaGómez et al. (2010). 


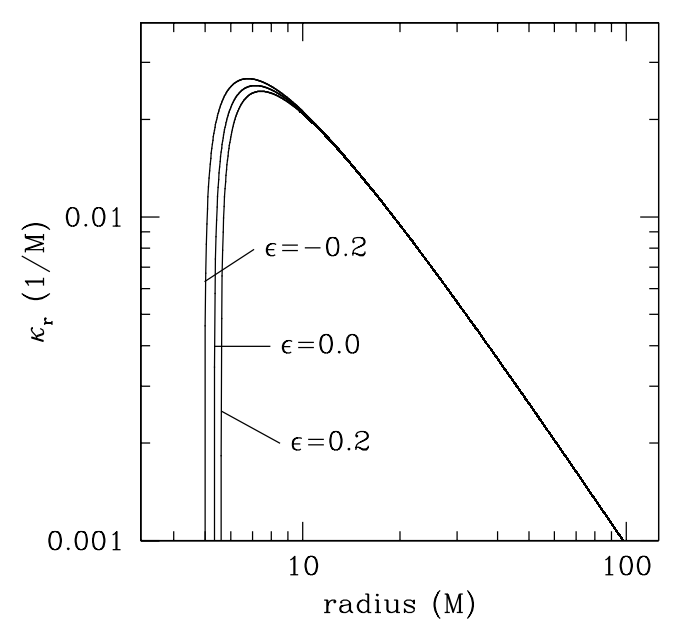

Figure 8.2 Radial epicyclic frequency $\kappa_{\mathrm{r}}$ versus radius for a particle orbiting a quasi-Kerr black hole with a spin of $a=0.2 \mathrm{M}$ and for several values of the parameter $\epsilon$. The radial epicyclic frequency increases with decreasing values of the radius and reaches a maximum at $r \approx 7 M$ for this spin. Decreasing values of the parameter $\epsilon$ shift the maximum to smaller radii.

I plot the radial epicyclic frequency $\kappa_{\mathrm{r}}$ as a function of radius for several values of the parameter $\epsilon$ and a spin of $a=0.2 M$ in Figure 8.2. The radial oscillation frequency increases with decreasing values of the radius and reaches a maximum at $r \approx 7 M$ for this spin. Decreasing values of the parameter $\epsilon$ shift the maximum to smaller radii.

In Figure 8.3 I plot the Lense-Thirring frequency

$$
\Omega_{\mathrm{LT}} \equiv \Omega_{\phi}-\Omega_{\theta}
$$

as a function of radius for several values of the parameter $\epsilon$ at a spin value $a=0.2 M$. The Lense-Thirring frequency describes the precession of the orbital plane of a particle moving around the black hole and vanishes in the case 


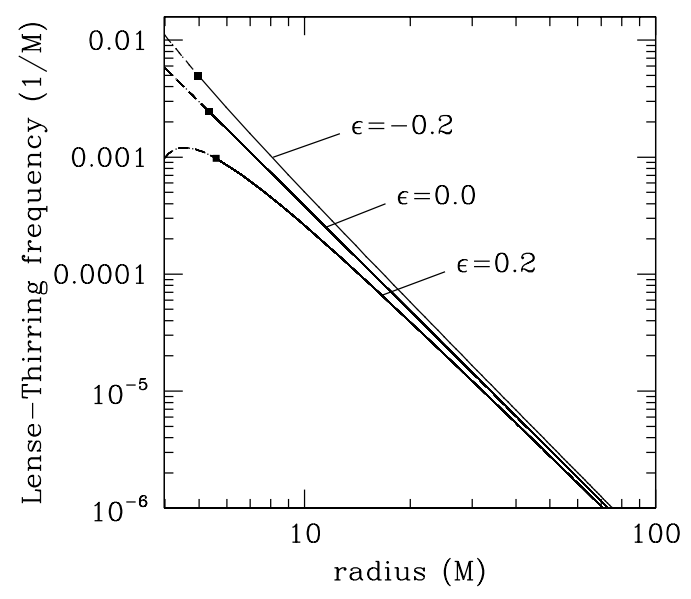

Figure 8.3 Lense-Thirring frequency $\Omega_{\mathrm{LT}}=\Omega_{\phi}-\Omega_{\theta}$ versus radius for a particle on an orbit around a quasi-Kerr black hole with a spin of $a=0.2 M$ and for several values of the parameter $\epsilon$. The Lense-Thirring frequency increases with decreasing values of the radius and of the parameter $\epsilon$. The dots mark the location of the ISCO, and dashed lines correspond to unstable orbits.

of a Schwarzschild black hole, where $\Omega_{\phi}=\Omega_{\theta}$. The Lense-Thirring frequency increases with decreasing values of the radius and of the parameter $\epsilon$. This frequency depends significantly on the parameter $\epsilon$ and changes by a factor of $\sim 5$ at the ISCO for values of the parameter $\epsilon= \pm 0.2$. Note that both the Lense-Thirring frequency and the radial oscillation frequency are significantly smaller than the Keplerian frequency $\Omega_{\phi}$.

\subsubsection{Stable Orbits in the Quasi-Kerr Spacetime}

In this section, I determine the location of the ISCO in the quasi-Kerr spacetime which separates the region of space where circular orbits are stable from the region where circular orbits are unstable. The location of this orbit, in turn, deter- 


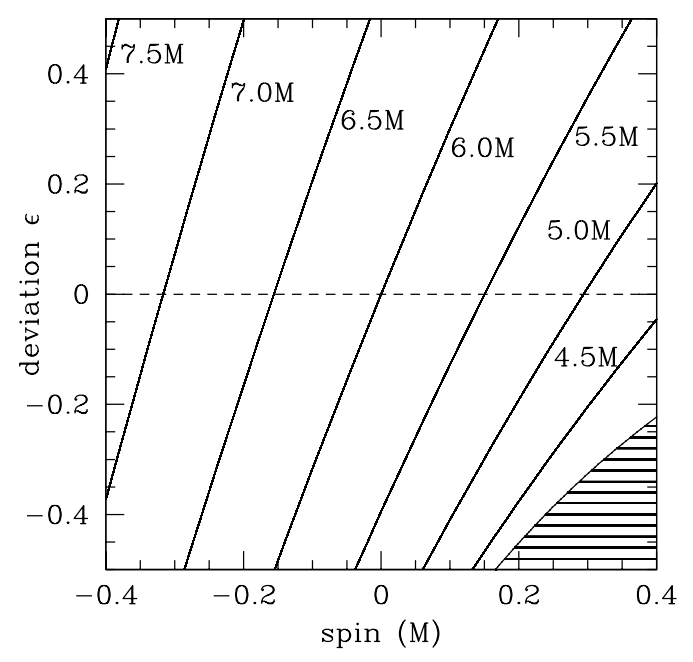

Figure 8.4 Location of the ISCO as a function of the spin $a$ and the parameter $\epsilon$. The radius of the ISCO decreases with increasing values of the spin and decreasing values of the parameter $\epsilon$. The dashed line corresponds to the special case of the Kerr metric, while the shaded region is excluded and marks the part of the parameter space where higher order terms in the radial epicyclic frequency become important.

mines the maximum Keplerian frequency for that particular central object.

In Chapter 2 I found the location of the ISCO from equation (8.10) by solving

$$
\frac{d E}{d r}=0
$$

In this chapter, I determine the location of the ISCO by computing the radius $r$ at which the frequency of small radial oscillations $\kappa_{\mathrm{r}}$ becomes imaginary, rendering circular equatorial motion unstable (see, also, Shibata \& Sasaki 1998; Berti \& Stergioulas 2004; Gair et al. 2008).

In Figure 8.4 I plot the location of the ISCO as a function of the spin and the parameter $\epsilon$. The location of the ISCO shifts to smaller radii for increasing values of the spin as in the case of Kerr black holes, while the radius of the ISCO 


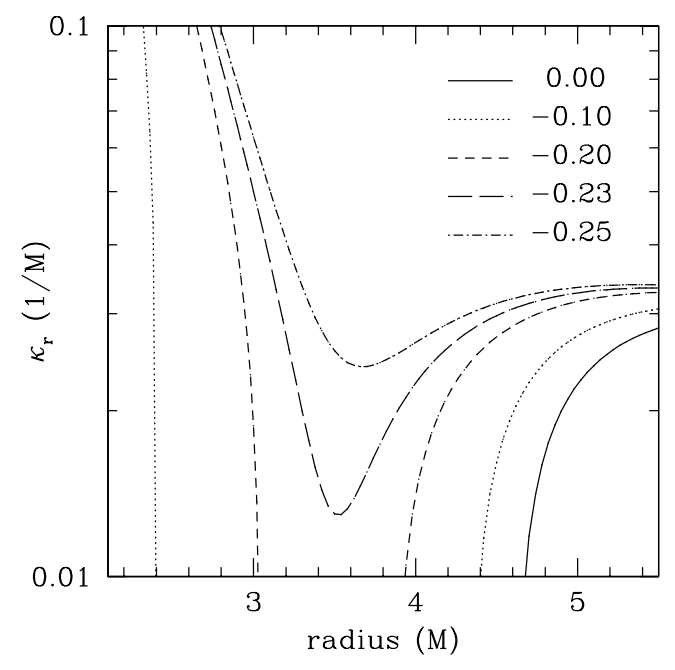

Figure 8.5 Radial epicyclic frequency $\kappa_{\mathrm{r}}$ as a function of radius for a black hole of spin $a=0.4 M$ and for several values of the parameter $\epsilon$. Zeros of the radial epicyclic frequency correspond to marginally stable orbits. Small negative values of the parameter $\epsilon$ lead to the emergence of a second marginally stable orbit in addition to the ISCO. For values of the parameter $\epsilon \lesssim-0.23$, these two merge and all orbits are now stable against radial perturbations. The ISCO is then determined by the onset of a vertical instability.

increases for increasing values of the parameter $\epsilon$. I denoted the location of the ISCO in Figures 8.1 and 8.3 by dots.

In addition to the region outside of the ISCO, I find a second branch of stable circular orbits similar to the ones reported in Gair et al. (2008). If the parameter $\epsilon$ is sufficiently negative for a given value of the spin, I observe the emergence of this branch at radii smaller than the radius of the ISCO as well as of a second unstable branch for which the epicyclic frequency $\Omega_{\theta}$ becomes imaginary.

I illustrate this behavior in Figure 8.5, where I plot the radial epicyclic frequency as a function of radius for a black hole with spin $a=0.4 M$ and for several 


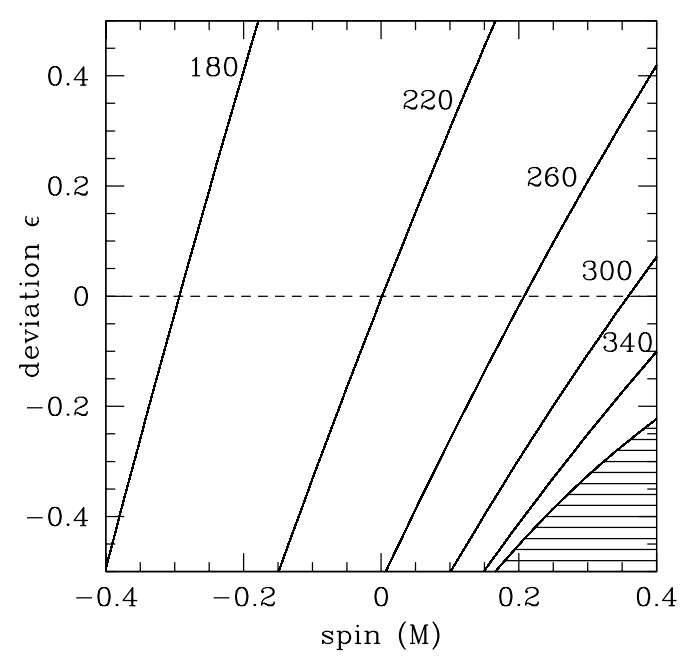

Figure 8.6 Maximal Keplerian frequency in Hz scaled appropriately for a $10 M_{\odot}$ mass black hole as a function of the spin $a$ and the parameter $\epsilon$. The maximal Keplerian frequency increases with increasing values of the spin and decreasing values of the parameter $\epsilon$. The dashed line corresponds to the special case of the Kerr metric. The shaded region marks the excluded part of the parameter space where higher order terms in the radial epicyclic frequency become important.

values of the parameter $\epsilon$. For a Kerr black hole, the radial epicyclic frequency has only one zero at a radius $r_{\mathrm{ISCO}} \approx 4.6 M$. If $\epsilon \lesssim 0$, the location of the ISCO decreases, while a second branch of stable circular orbits emerges in the neighborhood of the circular photon orbit. The radius of the second marginally stable circular orbit increases with decreasing values of the parameter $\epsilon$. For values of the parameter $\epsilon \lesssim-0.23$, the radial epicyclic frequency becomes strictly positive and these two marginally stable circular orbits disappear. In this case, all circular orbits are stable against radial perturbations, and the ISCO is determined by instability in the vertical direction.

Gair et al. (2008) referred to the larger one of these additional two marginally 
stable circular orbits as OSCO, the outermost stable circular orbit. In all cases, however, $r_{\mathrm{OSCO}}<r_{\mathrm{ISCO}}$. Since a particle on a circular orbit in this region has a larger energy and axial angular momentum than a particle on a circular orbit in the region $r \geq r_{\mathrm{ISCO}}$, the region bound by the OSCO, if present, is likely to be vacant of particles on circular orbits (see discussion in Gair et al. 2008). Generally, however, this region is very close to the black hole, where the quasi-Kerr metric is no longer perturbative (see Chapter 2).

The shaded region in Figure 8.4 marks the part of the parameter space where all circular equatorial orbits are radially stable. Albeit interesting in its own merit, I do not further consider this part of the parameter space, because higher order terms in the radial epicyclic frequency become important.

For particles in an accretion disk, circular motion at radii smaller than the ISCO is unstable, and the Keplerian frequency reaches its maximal value at the ISCO. The maximum Keplerian frequency is the largest oscillation frequency in the disk and, therefore, an upper bound on the dynamical frequencies. In Figure 8.6, I plot the maximal Keplerian frequency $\Omega_{\phi, \max }$ as a function of the spin $a$ and the parameter $\epsilon$. Since the Keplerian frequency depends only weakly on the parameter $\epsilon$ (see Figure 8.1), quadrupole deviations affect the maximal Keplerian frequency predominantly due to the shift of the ISCO. The shaded region, again, marks the excluded part of the parameter space where higher order terms in the radial epicyclic frequency become important. 


\subsection{Quasi-Periodic Oscillations}

In this section, I use my expressions of the Keplerian and epicyclic frequencies that I derived in Section 2 to demonstrate how QPOs observed in black-hole X-ray binaries can be used to test the no-hair theorem in two different scenarios. In both interpretations of detected QPO signals, the dynamical frequencies are important but play different roles. I argue that either one or two of the three parameters mass, spin, and quadrupole moment can be determined from a pair of QPOs and that the no-hair theorem can be tested in conjunction with an independent measurement of the remaining parameters.

\subsubsection{The Diskoseismology Model}

In general relativity, modes can be trapped in the accretion disk of a black hole giving rise to modulations of the measured flux. Detailed expressions of these modes have been derived in a hydrodynamic model in full general relativity (the diskoseismology model; Perez et al. 1997; Silbergleit et al. 2001). In this interpretation, the observed QPO pair can be identified as the lowest order gravity ( $g$-modes) and corrugation modes ( $c$-modes). The frequencies of these modes depend almost exclusively on the mass and spin in the case of a Kerr black hole and, consequently, both mass and spin can be determined from a pair of QPOs (e.g., Wagoner et al. 2001).

The radial epicyclic frequency $\kappa_{\mathrm{r}}$ has a maximum near the inner edge of the accretion disk (Okazaki et al. 1987). In Kerr geometry, the lowest order $g$-modes occur at frequencies that are very close to that maximum (Perez et al. 1997). Corrugation modes, on the other hand, can exist only in disks that corotate with the Kerr black hole with a spin $a$ in the range $0<a_{0} \leq a \lesssim 0.95$, where $a_{0} \sim 10^{-5}-10^{-3}$ 
(Silbergleit et al. 2001). The fundamental $c$-mode coincides with the LenseThirring frequency of the black hole evaluated at a radius $r_{\mathrm{c}}$ which is typically very close to the ISCO. For a black hole with a mass of $10 M_{\odot}$ and a disk luminosity $L \sim 10 \%$ of the Eddington luminosity, Silbergleit et al. (2001) estimate the radius $r_{\mathrm{c}}$ by the formula

$$
r_{\mathrm{c}}=r_{\mathrm{ISCO}}+K_{0} a^{-K_{1}}(1-a)^{K_{2}},
$$

where $K_{0}=0.093 M, K_{1}=0.79$, and $K_{2}=0.20$.

In a quasi-Kerr spacetime, the frequencies of the $g$ - and $c$-modes depend not only on the mass and spin of the black hole, but also on the quadrupole moment through the parameter $\epsilon$. Therefore, the no-hair theorem can be tested if either the mass or spin is measured independently, as I demonstrate in the following.

For my analysis, I make the simplifying assumptions that the frequency of the fundamental $g$-mode is equal to the maximum of $\kappa_{\mathrm{r}} / 2 \pi$ and that the fundamental $c$-mode is equal to the Lense-Thirring frequency of a quasi-Kerr black hole evaluated at the ISCO. For a Kerr black hole, the first assumption is an excellent approximation (Perez et al. 1997), while the second assumption provides an estimate that slightly deviates from the true value $\Omega_{\mathrm{LT}}\left(r_{\mathrm{c}}\right) / 2 \pi$. In this working definition, I also assume that quasi-Kerr $c$-modes exist as long as $\Omega_{\mathrm{LT}}\left(r_{\mathrm{ISCO}}\right)>0$. For a rigorous treatment, a detailed study of perturbations on accretion disks in quasi-Kerr spacetime is necessary, which is beyond the scope of this chapter.

In Figure 8.7, I plot as a function of the black hole spin (top panel) the fundamental frequencies of the $g$ - and $c$-modes of a Kerr black hole using the above working definition (solid lines) as well as the fundamental $c$-mode (dashed line) evaluated at the radius $r_{\mathrm{c}}$ given by equation (8.25). The curves for the $c$-mode 
deviate primarily at lower spins. In Figure 8.7 I also plot (bottom panel) this deviation between the expressions for the fundamental $c$-mode as a function of the spin $a$. In the range of spins that is relevant for this chapter, i.e., $0 \leq a / M \leq 0.4$, the deviation is $\sim 10-30 \%$ except for very low values of the spin $a \lesssim 0.1 M$.
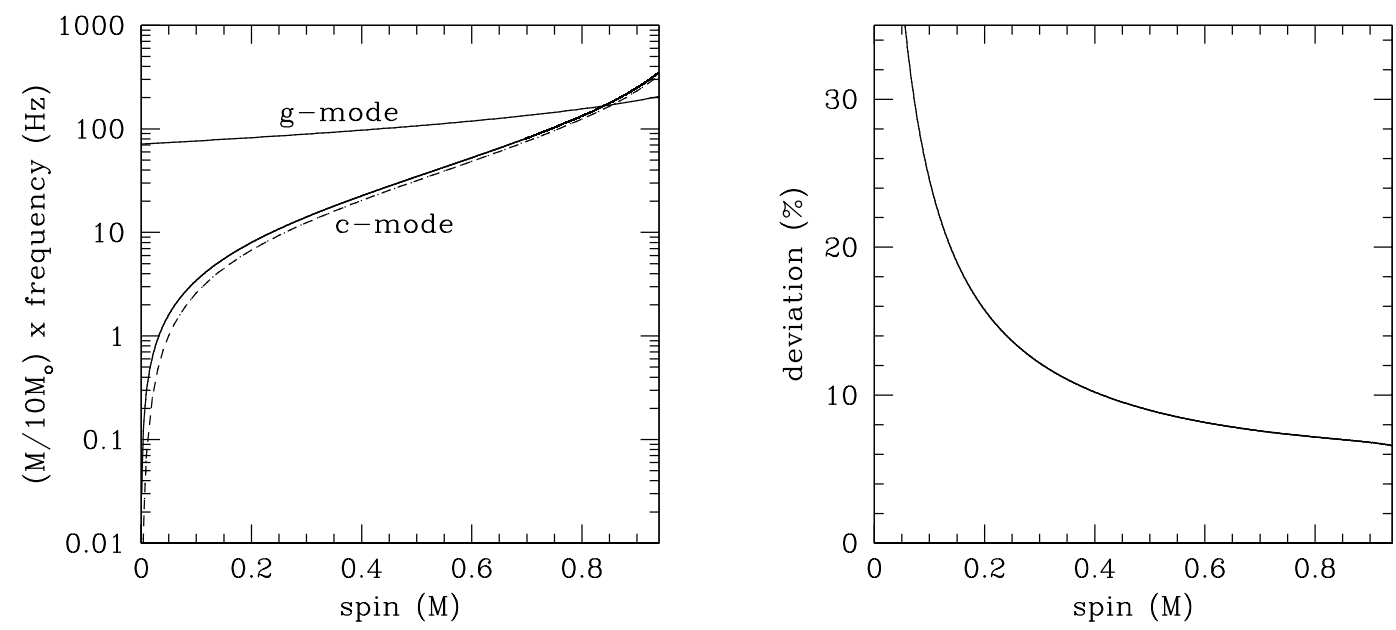

Figure 8.7 Left: Fundamental frequency of the $g$-mode and $c$-modes for a Kerr black hole as a function of spin. I evaluate the fundamental $c$-mode at the radius of the ISCO (solid line) and at the radius $r_{c}$ (dashed line). Right: Percent deviation between both expressions of the fundamental $c$-mode as a function of spin.

In Figure 8.8, I plot (top panel) the fundamental $g$-mode and (bottom panel) the fundamental $c$-mode as a function of spin for several values of the parameter $\epsilon$ in units of a $10 M_{\odot}$-black hole. In both cases, the frequency increases with increasing values of the spin, but decreases with increasing values of the parameter $\epsilon$.

In Figure 8.9, I plot frequency contours of the lowest order $g$-mode as a function of spin and the parameter $\epsilon$. The frequency of the fundamental $g$-mode in- 

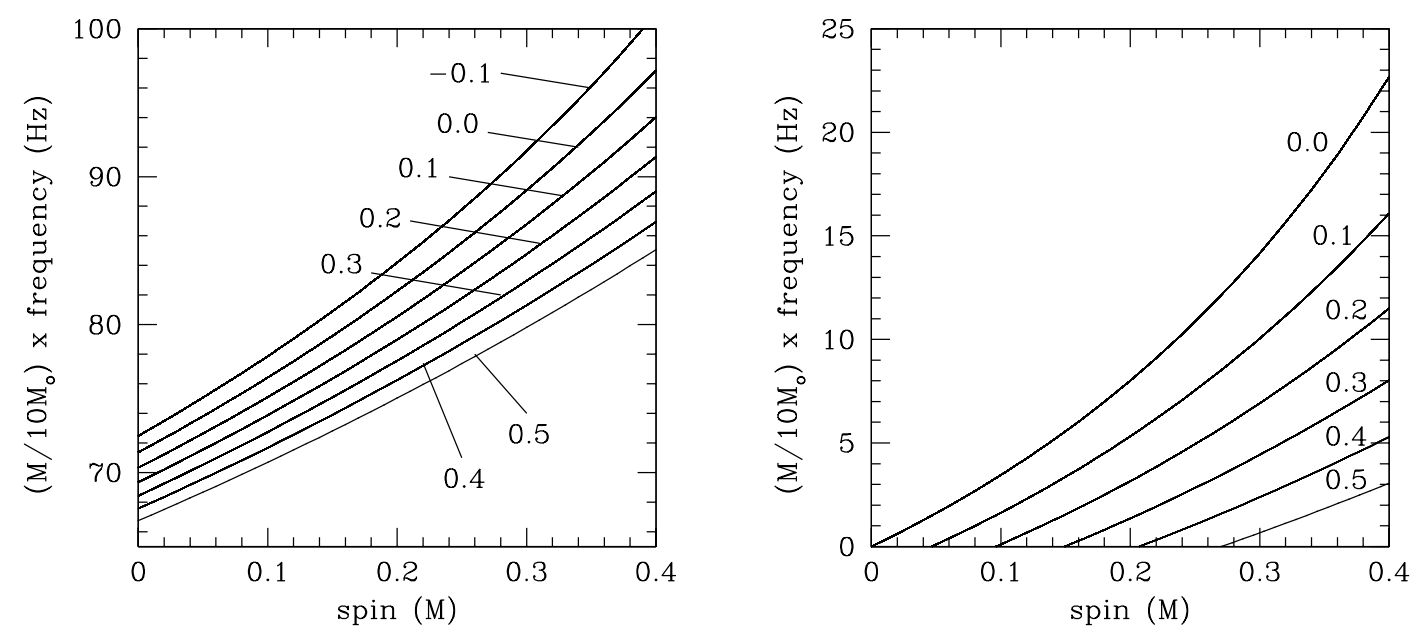

Figure 8.8 Frequencies of (left) the fundamental $g$-mode and (right) the fundamental $c$-mode scaled appropriately for a $10 M_{\odot}$-mass black hole as a function of spin for several values of the parameter $\epsilon$. Both the fundamental $g$-mode and $c$ mode frequencies increase with increasing values of the spin but decrease with increasing values of the parameter $\epsilon$.

creases for increasing values of the spin and decreasing values of the parameter $\epsilon$. The shaded region marks the excluded part of the parameter space.

As can be seen from Figure 8.9, contours of constant frequency of the fundamental $g$-mode are fairly linear in the spin $a$ and the parameter $\epsilon$. In order to further quantify this, I set $r \rightarrow r_{0}+\delta a+\eta \epsilon$, where $r_{0}=8 M$ is the location of the maximum radial epicyclic frequency for a Schwarzschild black hole and $\delta$ and $\eta$ are constants. Then, I expand the derivative of the radial epicyclic frequency given by expression (8.21) with respect to radius around its maximum $r=r_{0}$ for $a=\epsilon=0$ and solve the equation

$$
\left.\frac{d \kappa_{\mathrm{r}}^{2}}{d r}\right|_{r_{0}, a=\epsilon=0}+\left.\frac{d}{d a} \frac{d \kappa_{\mathrm{r}}^{2}}{d r}\right|_{r_{0}, a=\epsilon=0} a+\left.\frac{d}{d \epsilon} \frac{d \kappa_{\mathrm{r}}^{2}}{d r}\right|_{r_{0}, a=\epsilon=0} \epsilon=0
$$




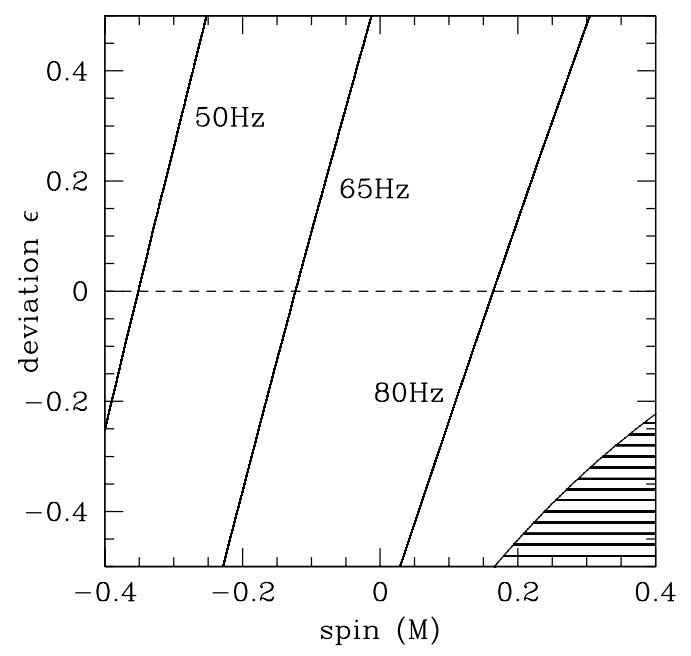

Figure 8.9 Frequency contours of the fundamental $g$-mode as a function of the spin $a$ and the parameter $\epsilon$ scaled for a $10 M_{\odot}$-black hole. The fundamental $g$ mode frequency increases with increasing values of the spin and decreasing values of the parameter $\epsilon$. The shaded region is excluded from the parameter space.

for the parameters $\delta$ and $\eta$. I obtain the solutions

$$
\begin{gathered}
\delta=-\frac{47}{8 \sqrt{2}}, \\
\eta=-\frac{5}{72}\left[34128 \ln \left(\frac{4}{3}\right)-9835\right] M .
\end{gathered}
$$

From these expressions, I derive a linear approximation of the fundamental $g$ mode frequency in terms of the spin $a$ and the parameter $\epsilon$ given by the equation

$$
\begin{gathered}
\kappa_{\mathrm{r}, \max }^{2}=\frac{1}{M^{2}} \\
\times\left[\frac{1}{2048}+\frac{15}{16384 \sqrt{2}} \frac{a}{M}-\frac{5\left[5400 \ln \left(\frac{4}{3}\right)-1553\right]}{16384} \epsilon\right] \\
\approx\left(5.09+6.75 a^{*}-1.54 \epsilon\right) \times 10^{3}\left(\frac{M}{10 M_{\odot}}\right)^{-2} \mathrm{~Hz}^{2},
\end{gathered}
$$




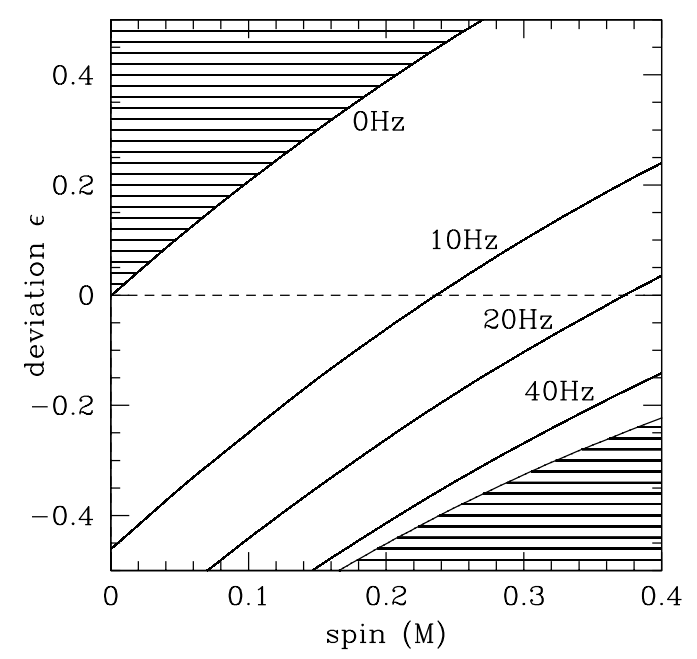

Figure 8.10 Frequency contours of the fundamental $c$-mode as a function of the spin $a$ and the parameter $\epsilon$ scaled for a $10 M_{\odot}$-black hole. The fundamental $c$-mode frequency increases with increasing values of the spin and decreasing values of the parameter $\epsilon$. The shaded region is excluded from the parameter space.

where $a^{*} \equiv a / M$. This approximation coincides with the maximum of the full expression of the radial epicyclic frequency given by equation (8.21) to within $10 \%$ across the full range of the values of the spin and the parameter $\epsilon$ considered in Figure 8.9.

In Figure 8.10, I plot frequency contours of the fundamental $c$-modes as a function of spin and the parameter $\epsilon$. The $c$-mode frequency rises with increasing values of the spin and decreasing values of the parameter $\epsilon$. The shaded regions mark the excluded parts of the parameter space. The top left corner is excluded according to my requirement that quasi-Kerr $c$-modes only exist in corotating disks. Since the Lense-Thirring frequency given by equation (8.23) is the difference between the epicyclic frequencies in the $\phi$ and $\theta$ coordinates, its main 
dependence is on the parameter $\epsilon$.

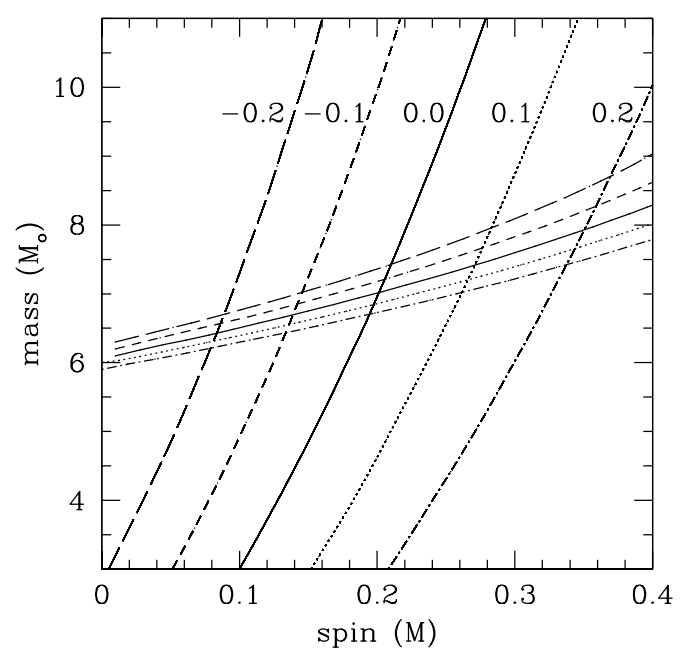

Figure 8.11 Lines of constant $g$ - and $c$-mode frequency (at $117.5 \mathrm{~Hz}$ and $11.5 \mathrm{~Hz}$, respectively) as a function of mass and spin for several values of the parameter $\epsilon$. The intersection points of each corresponding pair of lines marks the particular combination of mass and spin for a given value of the parameter $\epsilon$.

In Figures 8.11 and 8.12 I illustrate how measurements of $g$-and $c$-modes of a particular black hole can be used to test the no-hair theorem. For this purpose, I choose a black hole with a mass of $M=7 M_{\odot}$ and a spin of $a=0.2 M$. According to equations (8.21) and (8.23), the corresponding frequencies of the $g$-mode and the $c$-mode, respectively, are $117.5 \mathrm{~Hz}$ and $11.5 \mathrm{~Hz}$. In the following, I assume that these frequencies have been measured and identified as the respective lowest order $g$ - and $c$-modes.

In Figure 8.11, I plot contours of constant $g$ - and $c$-modes for frequencies of $117.5 \mathrm{~Hz}$ and $11.5 \mathrm{~Hz}$, respectively, as a function of the black hole mass $M$ and spin $a$ for several values of the parameter $\epsilon$. The intersection point of each pair of lines 


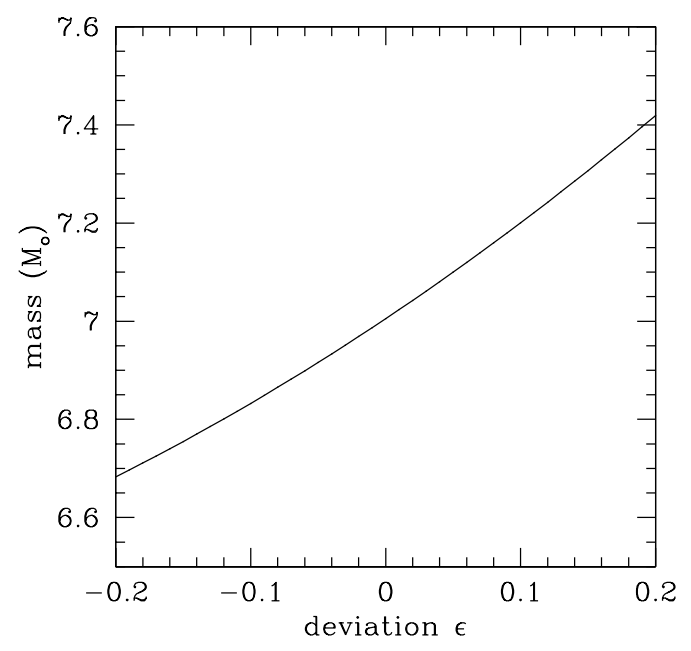

Figure 8.12 Dependence of the black hole mass on the parameter $\epsilon$ determined by the intersection points as illustrated in Figure 8.11. Increasing values of the parameter $\epsilon$ correspond to a larger mass. A separate measurement of the mass breaks the degeneracy of the mass and the parameter $\epsilon$ allowing for a test of the no-hair theorem.

marks the particular combination of the parameters $M, a$, and $\epsilon$ that is consistent with this hypothetical measurement. Increasing values of the parameter $\epsilon$ shift the intersection point to larger values of the mass and spin. In the case of a Kerr black hole, this reproduces the assumed values for the mass and spin.

Since I have three free parameters and two measurements, I can express the mass in terms of the parameter $\epsilon$. In Figure 8.12, I plot the mass as a function of the parameter $\epsilon$ for the intersection points given in Figure 8.11. Increasing values of the parameter $\epsilon$ correspond to a larger black hole mass. In combination with an independent mass measurement, the degeneracy between the mass and the parameter $\epsilon$ is broken, and all three parameters mass, spin, and quadrupole moment of the black hole can be determined. This, in turn, allows us to test the 


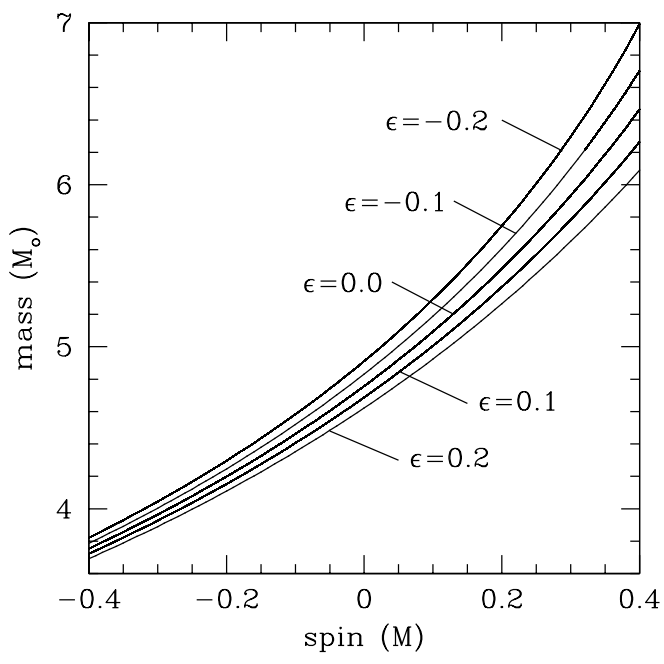

Figure 8.13 Spin versus mass of a black hole for a QPO pair at frequencies $300 \mathrm{~Hz}$ and $450 \mathrm{~Hz}$ in 1:2 resonance for several values of the parameter $\epsilon$. The mass increases for increasing values of the spin and decreasing values of the parameter $\epsilon$.

no-hair theorem via relation (2.5).

\subsubsection{The Kinematic Resonance Model}

QPOs can also be modeled as resonances among the Keplerian and epicyclic frequencies (Kluźniak \& Abramowicz 2001; Abramowicz et al. 2003). Several pairs of QPOs have been observed in galactic black holes at a ratio of $\approx 3 / 2$ (see Remillard \& McClintock 2006). The assumption of either a 1:2 or a 1:3 resonance between the Keplerian frequency and the radial epicyclic frequency for the observed QPO pair in the binary GRO J1655-40 has lead to a determination of the spin of this black hole (Abramowicz \& Kluźniak 2001).

I demonstrate the potential of this approach to test the no-hair theorem by considering an example similar to GRO J1655-40. I follow the analysis in Abramow- 


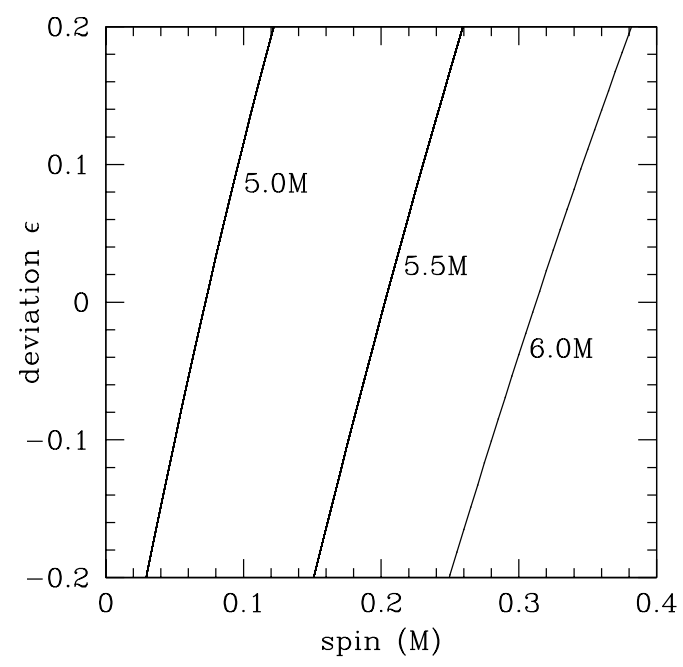

Figure 8.14 Spin versus the parameter $\epsilon$ for the intersection points of the lines in Figure 8.13 for different values of the mass $M$. The degeneracy between spin and the parameter $\epsilon$ can be broken by an independent spin measurement.

icz \& Kluźniak (2001), but I consider only the range of spins $-0.4 \leq a / M \leq 0.4$ in order to be consistent with my framework. For a 1:2 resonance between the Keplerian and radial epicyclic frequencies, I set $\Omega_{\phi}=300 \mathrm{~Hz}$ and $\kappa_{\mathrm{r}}=150 \mathrm{~Hz}$. The resonance occurs at a radius $r_{\text {res }}$ which is a function of mass and spin for a Kerr black hole and of mass, spin, and the parameter $\epsilon$ for a quasi-Kerr black hole.

In Figure 8.13, I plot the mass as a function of spin for $\Omega_{\phi}$ and $\kappa_{\mathrm{r}}$ in a 1:2 resonance for several values of the parameter $\epsilon$. The mass increases for increasing values of the spin and decreasing values of the parameter $\epsilon$. If the mass of the central object is known from an independent measurement, then only certain combinations of the spin and the parameter $\epsilon$ are consistent with such a measurement. For this illustration, I assume hypothetical mass measurements of $M=5.0,5.5,6.0 M_{\odot}$, respectively. 
In Figure 8.14, I plot the parameter $\epsilon$ as a function of spin for the intersection points of the lines in Figure 8.13 with these hypothetical mass measurements. Higher values of the spin correspond to larger values of the parameter $\epsilon$ for a given black-hole mass. The degeneracy between spin and quadrupole moment can be broken by an independent spin measurement from, e.g., relativistically broadened iron lines (e.g., Brenneman \& Reynolds 2009), which in turn would uniquely determine the parameter $\epsilon$. In this QPO model, therefore, the no-hair theorem can be tested in conjunction with additional measurements of mass and spin. 


\subsection{Conclusions}

In this chapter, I derived expressions of the Keplerian and epicyclic frequencies in the quasi-Kerr spacetime and explored their properties. I showed that, for moderate values of the spin, the Keplerian frequency depends almost exclusively on the mass and spin of a given black hole, while the radial and vertical epicyclic frequencies depend significantly on the value of the parameter $\epsilon$ near the inner edge of the accretion disk in addition to their dependence on the mass and spin. I determined the location of the ISCO for the ranges of the parameters $-0.4 \leq a / M \leq 0.4$ and $-0.5 \leq \epsilon \leq 0.5$ and showed that the Lense-Thirring frequency for a particle at the ISCO changes by a factor of $\sim 5$ for a black hole with spin $a=0.2 \mathrm{M}$ as the parameter $\epsilon$ is varied from -0.2 to +0.2 . I discussed the emergence of two additional stable orbits inside the ISCO and possible consequences for astrophysical black holes.

I demonstrated how this approach can be applied to QPOs observed in galactic stellar-mass black holes and AGN focusing on two different models. If a pair of QPOs is observed and identified as the fundamental $g$ - and $c$-modes, respectively, the mass, spin, and quadrupole moment of that black hole can be measured if the mass is known from dynamical observations. If the QPO pair is viewed as a nonlinear resonance between the Keplerian and epicyclic frequencies, the parameter $\epsilon$ can be measured together with independent measurements of the mass and spin.

In addition to galactic black holes, Sgr $\mathrm{A}^{*}$ is another prime target for tests of the no-hair theorem. High-resolution observations of stars in close orbit around Sgr $A^{*}$ for over a decade have lead to a precise mass and distance measurement of Sgr A* (Ghez et al. 2008; Gillessen et al. 2009). Recent VLBI observations 
resolved Sgr A* on horizon scales (Doeleman et al. 2008) and pointed the way towards the first image of Sgr $\mathrm{A}^{*}$ in the near future (Fish \& Doeleman 2009). In Chapter 6, I showed that a ring of light will surround the image of a black hole and that its shape depends directly on the mass, spin, quadrupole moment, and inclination of the black hole. In particular, I showed that the diameter depends predominantly on the mass, while the displacement and the asymmetry of this ring are proportional to the quantities $a \sin i$ and $\epsilon \sin ^{3 / 2} i$, respectively, where $i$ is the inclination angle of the angular momentum of the black hole with respect to a distant observer. I noted that one additional observable is required to break the degeneracy of the displacement and the asymmetry with the inclination.

Here I argue that a full test of the no-hair theorem for Sgr A* may be accomplished with a combination of VLBI imaging of the ring of light (or, more generally, the shadow; see Chapter 6) and the observation of quasi-periodic variability in the emission from Sgr $A^{*}$ using VLBI techniques (Doeleman et al. 2009a). Such variability is thought to arise from density fluctuations orbiting around the center of mass. The orbital frequency of these inhomogeneities is the Keplerian frequency $\Omega_{\phi}$. In this chapter, I showed that, for moderate values of the spin, the dependence of $\Omega_{\phi}$ on the parameter $\epsilon$ is negligible. Consequently, if highfrequency VLBI observations of Sgr A* (Doeleman et al. 2009a) can measure the Keplerian frequency of an orbiting hot spot and if Sgr A* is not spinning rapidly, they will measure the spin of Sgr $A^{*}$ irrespectively of the particular value of the parameter $\epsilon$. Therefore, measurements of the displacement and the asymmetry of the ring of light (or the shadow) of Sgr $\mathrm{A}^{*}$ will uniquely determine the inclination and the parameter $\epsilon$ allowing us to test the no-hair theorem. 


\section{CHAPTER 9}

\section{RELATIVISTICALLY BROADENED IRON LINES}

In this chapter, I analyze the prospects of using observations of fluorescent iron line profiles to test the no-hair theorem. In Chapter 5, I developed a high-performance ray-tracing algorithm and simulated first iron line profiles using the quasiKerr metric (Glampedakis \& Babak 2006). I showed that these profiles can depend significantly on potential deviations from the Kerr metric. The quasi-Kerr metric, however, can only be used for moderately spinning black holes with spins $a \lesssim 0.4 M$ due to the existence of pathologies near the event horizon (Johannsen \& Psaltis 2010a; Johannsen et al. 2012b). Since most of the observed iron lines indicate high values of the spins (see, e.g., Miller 2007), my initial approach cannot be readily applied to comparing models with data.

In Chapter 4, I constructed a Kerr-like black hole metric that is regular everywhere outside of the event horizon and, thus, suitable for the description of rapidly spinning black holes (Johannsen \& Psaltis 2011c; hereafter JP11). Similar to the quasi-Kerr metric, my metric depends on an additional parameter $\epsilon_{3}$ and coincides with the Kerr metric if $\epsilon_{3}=0$. Here, I incorporate my new black hole metric into my algorithm and simulate iron line profiles over the entire range of spins.

I demonstrate that deviations from the Kerr metric lead to shifts of the measured flux primarily at high energies as well as in the low-energy tail of the line profiles. I show that these changes can be significant for a black hole with a fixed value of the spin and estimate the required precision of future $\mathrm{X}$-ray missions in order to be able to test the no-hair theorem with fluorescent iron lines for disks of 
different inclinations.

I also show, however, that line profiles from black holes with different spins and values of the parameter $\epsilon_{3}$, chosen in such a way that the spacetimes have the ISCO at the same coordinate radius, are practically indistinguishable even for black holes with high spins. I isolate this effect from the other relativistic properties of the spacetime and show that these properties only contribute to the line shape at the few percent level. I argue that this correlation may be reduced in combination with other observables such as the frequencies of quasi-periodic oscillations (QPOs), which depend only indirectly on the location of the ISCO. The contents of this chapter have been submitted to The Astrophysical Journal as Johannsen \& Psaltis (2012). 


\subsection{Testing the No-Hair Theorem with Fluorescent Iron Lines}

In this section, I simulate profiles of relativistically broadened fluorescent iron lines from a geometrically thin accretion disk located in the equatorial plane of a black hole that is described by my metric (JP11). I study signatures of potential deviations from the Kerr metric in terms of the free parameter $\epsilon_{3}$. I summarize the explicit form of this metric as well as some of its properties in Appendix B.

The geometry is the same as in Psaltis \& Johannsen (2012) and Johannsen \& Psaltis (2010b). I consider an observer at a large distance $d$ and an inclination angle $\theta_{0}$ from the rotation axis of the black hole. I define an image plane with Cartesian coordinates $\left(\alpha_{0}, \beta_{0}\right)$ that is centered at $\phi=0$ and is perpendicular to the line of sight of the observer. I trace rays from the image plane to the equatorial plane using a forth-order Runge-Kutta integrator of the geodesic equations.

I model the accretion disk as having a radial extent from the ISCO to some outer radius $r_{\text {out }}$. I calculate the location of the ISCO by numerically finding the minimum of the orbital energy $E$ given by Equation (B.6). For positive values of the parameter $\epsilon_{3}$, the ISCO lies closer to the event horizon relative to the location of ISCO in the Kerr metric for a black hole with the same spin. For negative values of the parameter $\epsilon_{3}$, the ISCO lies further away from the event horizon (JP11). For $\epsilon_{3}=0$, I use the analytic expression for the location of the ISCO in the Kerr metric from Bardeen (1973).

I assume that plasma moves on circular orbits in the disk at the local Keplerian velocity $u=\left(u^{t}, 0,0, u^{\phi}\right)$, where the velocity components $u^{t}$ and $u^{\phi}$ are given by the expressions in Equation (B.16). I also assume that the disk emission is monochromatic at a rest frame energy $E_{0} \approx 6.4 \mathrm{keV}$ and isotropic with an emissivity $\varepsilon(r) \propto r^{\alpha}$. 

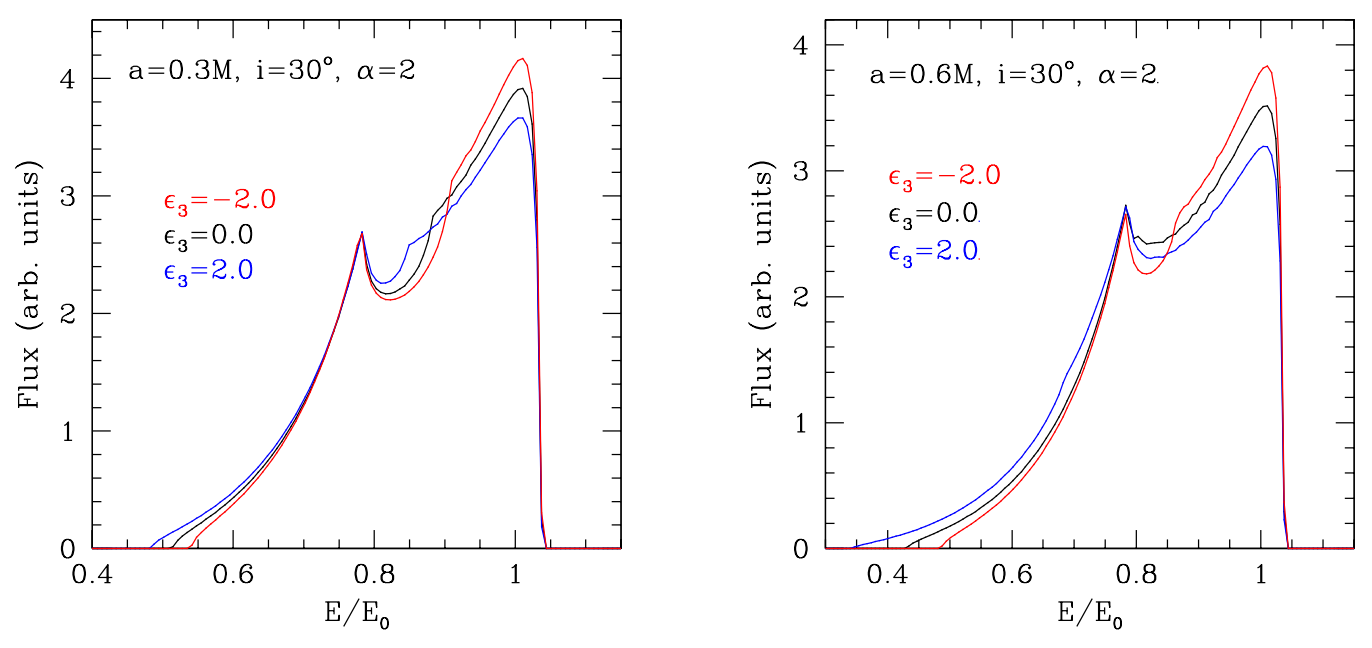

Figure 9.1 Profiles of fluorescent iron lines for black holes with spin (top) $a=$ $0.3 \mathrm{M}$ and (bottom) $a=0.6 \mathrm{M}$, inclination $\theta_{0}=30^{\circ}$ and emissivity index $\alpha=2$ for several values of the parameter $\epsilon_{3}$ that measures the deviation from the Kerr metric. Modifications of the profiles occur primarily in the high-energy peak and the low-energy tail.

The parameter $\epsilon_{3}$ likewise affects the lightbending experienced by the emitted photons (see Figure 9.3; Johannsen 2012) as well as the shift of the photon energy due to Doppler boosting, relativistic beaming, and the gravitational redshift, which is given by the expression

$$
g \equiv \frac{E_{\mathrm{im}}}{E_{\mathrm{d}}}=\frac{\left(g_{\mu \nu} k^{\mu} u^{\nu}\right)_{\mathrm{im}}}{\left(g_{\mu \nu} k^{\mu} u^{\nu}\right)_{\mathrm{d}}}
$$

In this expression, the subscripts "im" and " $\mathrm{d}$ " refer to the image plane and the accretion disk, respectively, $k^{\mu}$ is the photon 4-momentum (given explicitly in Johannsen \& Psaltis 2010b), and I set the 3-velocity of the observer at the image plane to zero.

I then compute the observed specific intensity $I\left(\alpha_{0}, \beta_{0}\right)$ at each point in the 

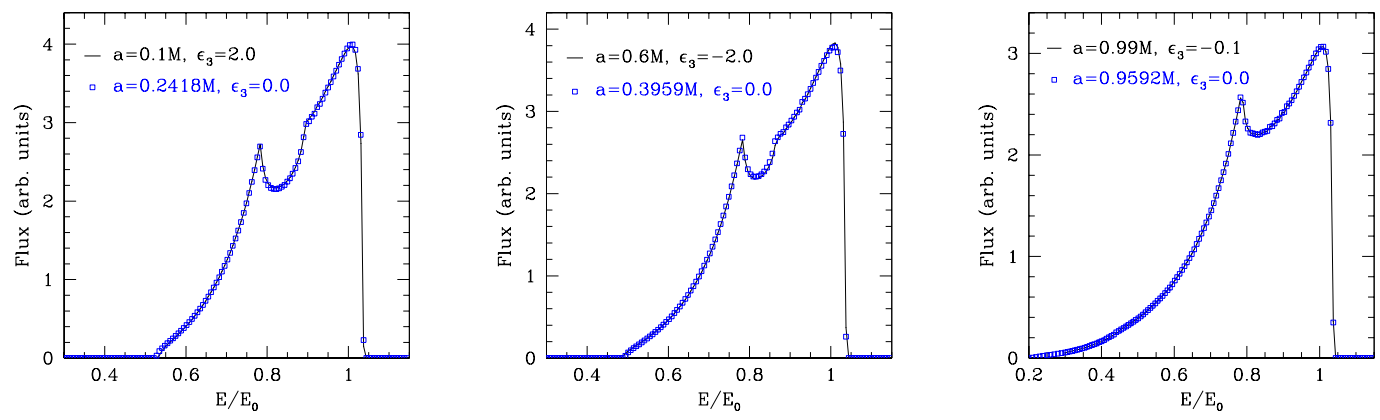

Figure 9.2 Iron line profiles for several pairs of the spin and values of the parameter $\epsilon_{3}$ that correspond to black holes with the same ISCO radius. The profiles are practically indistinguishable even at a very high spin. In the latter case, however, the range of pairs of the spin and values of the parameter $\epsilon_{3}$ with the same ISCO is much smaller than at low spins.

image plane using the Lorentz invariant $I / E_{p}^{3}$, where $I$ and $E_{p}$ are the specific intensity and the energy of the emitted photons, respectively. I obtain the total observed specific flux as

$$
F_{E}=\frac{1}{d^{2}} \int d \alpha_{0} \int d \beta_{0} I\left(\alpha_{0}, \beta_{0}\right)\left[E_{p}-E_{0} g\left(\alpha_{0}, \beta_{0}\right)\right]
$$

I calculate this two-dimensional integral with a Monte Carlo integration over the image plane using a set of narrow energy bins. I simulate the iron line profiles for black holes with spin values $0 \leq a / M \leq 1$ and values of the parameter $-2 \leq \epsilon_{3} \leq 2$ as long as the event horizon is entirely closed (see discussion in JP11).

In Figure 9.1, I plot iron line profiles for several values of the spin and the parameter $\epsilon_{3}$ at an inclination $\theta_{0}=30^{\circ}$, emissivity index $\alpha=2$, and outer disk radius $r_{\text {out }}=15 M$. The effect of changing the parameter $\epsilon_{3}$ is two-fold. First, the flux of the "blue" peak of the iron line centered at $E / E_{0} \sim 1$ is augmented/diminished for decreasing/increasing values of the parameter $\epsilon_{3}$. This effect is caused pre- 


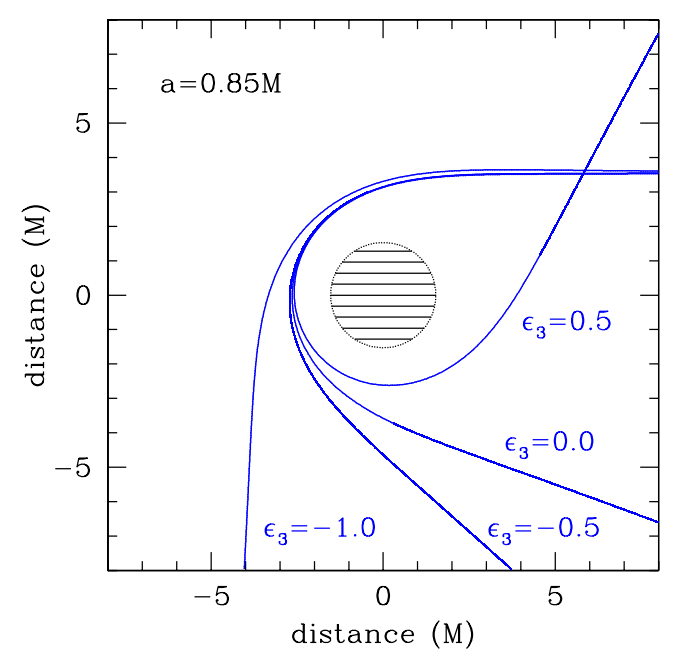

Figure 9.3 Trajectories of photons lensed by a black hole with a (counterclockwise) spin $a=0.85 \mathrm{M}$ for several values of the parameter $\epsilon_{3}$. The shaded region corresponds to the event horizon of a Kerr black hole of equal spin.

dominantly by the orbital velocity of the accretion flow, which differs from the corresponding orbital velocity in the Kerr metric at the same radius. At the same time, the flux of the "red" peak centered at $E / E_{0} \sim 0.75$ is affected only marginally.

While the absolute flux depends on the particular observation, the relative flux between the two peaks is specific to the value of the parameter $\epsilon_{3}$ and, therefore, a signature of a potential violation of the no-hair theorem. Such a modification of the flux ratio of the two peaks can also be achieved by changing the emissivity index $\alpha$ or the outer disk radius $r_{\text {out }}$. The index $\alpha$ has to be determined simultaneously from a spectral fit of the line profile. Changing the outer disk radius, however, shifts the positions of the peaks and can, thus, be disentangled from the effect of a deviation from the Kerr metric on the relative peak fluxes. 
Second, due to the shift of the location of the ISCO, increasing/decreasing values of the parameter $\epsilon_{3}$ lead to an extended/shortened "red tail" of the iron line at low energies, which is produced by photons emitted at radii near the ISCO that experience the corresponding strong gravitational redshift.

Since the ISCO in my spacetime depends on both the spin and the parameter $\epsilon_{3}$, a measurement of the ISCO radius alone can only constrain certain combinations of the spin and the parameter $\epsilon_{3}$. In Psaltis \& Johannsen (2012), I showed that the entire iron line profiles in the quasi-Kerr metric (Glampedakis \& Babak 2006) are practically indistinguishable for different combinations of the spin and the deviation parameter that have the ISCO at the same coordinate radius, but I had to limit my analysis to spin values $a \lesssim 0.4 M$.

Here, using my new metric, I perform a similar analysis covering low, intermediate, and high spins. In Figure 9.2, I plot iron line profiles for three such pairs of the spin and the parameter $\epsilon_{3}$ that have the same ISCO. Even in the case of a very high spin $a=0.99 \mathrm{M}$ the line profiles are practically indistinguishable. Note, however, that constraints of pairs of spins and values of the parameter $\epsilon_{3}$ at such high spin values will still be tight, because the range of pairs of spins and values of the deviation parameter that correspond to the same ISCO radius is much smaller than at low spins (see Figure 5 in JP11). 
9.2 Precision Requirements for Measurements of Potential Violations of the NoHair Theorem
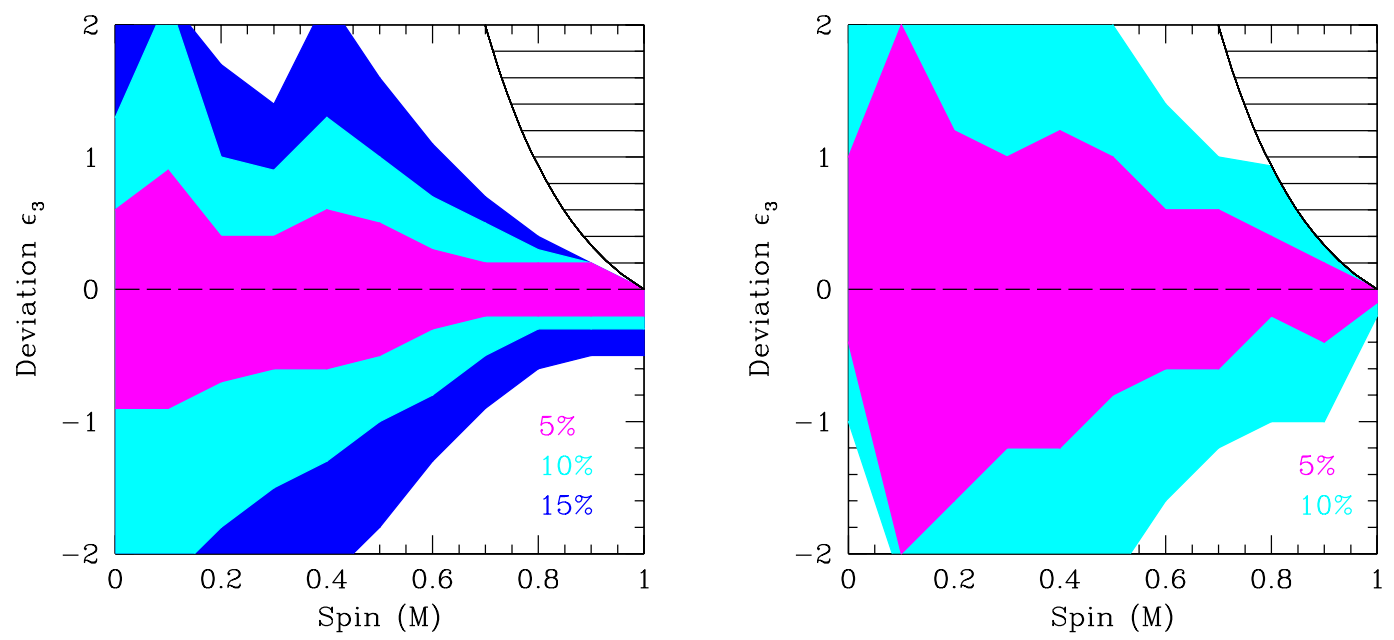

Figure 9.4 Contours of the precision required in future observations of iron lines to place constraints on deviations from the Kerr metric, for different values of the black-hole spin. Top: $\theta_{0}=30^{\circ}$. Bottom: $\theta_{0}=60^{\circ}$. The precision requirements are lower at higher spins thanks to the sensitive dependence of the line profiles on the deviation parameter $\epsilon_{3}$. At an inclination $\theta_{0}=30^{\circ}$, the effect of changing the deviation parameter $\epsilon_{3}$ is generally larger compared to the effect at an inclination $\theta_{0}=60^{\circ}$. In the former case, constraints $\left|\epsilon_{3}\right| \lesssim 1$ can be obtained at a precision level of $\sim 5 \%$ level irrespective of the value of the spin. The shaded region marks the part of the parameter space, where the central object of the spacetime is no longer a black hole (see JP11).

In this section, I estimate the required precision that observations with future X-ray missions have to achieve in order to measure potential deviations from the Kerr metric from fluorescent iron line profiles. I define the relative difference $\sigma$ between a line profile with a nonzero value of the parameter $\epsilon_{3}$ from the Kerr line 
profile with otherwise identical parameters by the expression

$$
\sigma \equiv \frac{1}{N} \sqrt{\sum_{i=1}^{N} \frac{\left(F_{i}^{\mathrm{dev}}-F_{i}^{K}\right)^{2}}{\left(F_{i}^{K}\right)^{2}}},
$$

where $N$ is the number of energy bins, and $F_{i}^{K}$ and $F_{i}^{\text {dev }}$ are the flux of the $i$ th bin of the line in the Kerr metric and the metric JP11, respectively. Here I use $N \sim 100$, although the actual value affects the results only marginally.

In Figure 9.4, I plot contours of the relative difference $\sigma$ over the ranges $0 \leq$ $a / M \leq 1$ and $-2 \leq \epsilon_{3} \leq 2$ for inclination angles $\theta_{0}=30^{\circ}$ and $\theta_{0}=60^{\circ}$. In both cases, I held the other parameters fixed at values $\alpha=2$ and $r_{\text {out }}=15 \mathrm{M}$.

The relative difference is generally larger at lower spins, and at a given level of measured precision constraints on the spin and deviation parameter of the spacetime are significantly more tight for the lower inclination $\theta_{0}=30^{\circ}$. In this case, a precision of $\sim 5 \%$ leads to constraints on the parameter $\left|\epsilon_{3}\right|$ to less than order unity at any spin value. In contrast to the relatively strong alterations of the entire line profile at $\theta_{0}=30^{\circ}$, at $\theta_{0}=60^{\circ}$, the difference arises mainly from the flux of the "blue" peak. In Figure 9.5, I demonstrate this effect for a black hole with spin $a=0.2 M$ and $\alpha=2$ and for several values of the parameter $\epsilon_{3}$.

In order to isolate the shift of the ISCO as a function of the parameter $\epsilon_{3}$ at a given spin from the other effects that change the lightbending and energy of the emitted photons as I described in the previous section, I calculated the relative difference between line profiles as a function of the black hole spin and the parameter $\epsilon_{3}$ for disks that have the same inner disk radius. Since the location of the ISCO itself is coordinate-dependent, I fixed the inner disk edge at the radius, where the Keplerian frequency of the modified spacetime (JP11) as observed at infinity equals the Keplerian frequency of the Kerr spacetime evaluated at the 


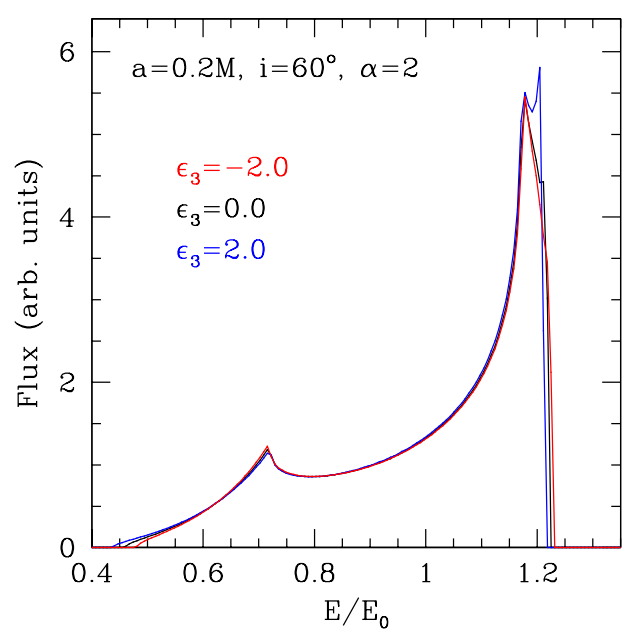

Figure 9.5 Line profiles for a black hole with a spin $a=0.2 M$, inclination $\theta_{0}=60^{\circ}$, and emissivity index $\alpha=2$. Modifications of the profile manifest primarily in the high-energy peak.

same spin and (Kerr) ISCO radius (see Figure 9.7).

In Figure 9.6, I plot contours in the $\left(a, \epsilon_{3}\right)$-plane of the relative precision $\sigma$ with respect to Kerr line profiles for $\theta_{0}=30^{\circ}, \alpha=2$, and $r_{\text {out }}=15 M$. Deviations $\left|\epsilon_{3}\right| \sim 1$ correspond to a relative difference of roughly $2 \%$. This implies that a line profile has to be measured with an accuracy of a few percent in order to measure deviations from the Kerr metric from fluorescent iron line profiles alone. Such accuracy is difficult to achieve given the systematic uncertainties in modeling the emissivity profile of the accretion disk. Therefore, observations of iron line profiles alone can only place constraints on pairs of spins and values of the parameter $\epsilon_{3}$ that correspond roughly to the same ISCO radius. A measurement of both parameters requires an independent measurement of the spin and the parameter $\epsilon_{3}$ from, e.g., quasi-periodic variability. 


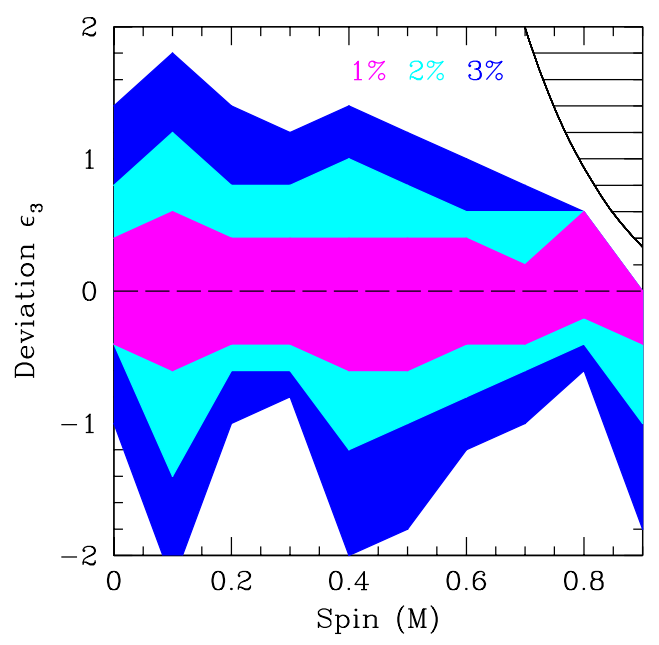

Figure 9.6 Contours of the relative difference between line profiles for $\theta_{0}=30^{\circ}$ and $\alpha=2$ as a function of spin and the parameter $\epsilon_{3}$, holding the inner edge of the accretion disk fixed at the radius where the Keplerian frequency equals the Kerr Keplerian frequency for the same spin at the Kerr ISCO. The relativistic effects that change the lightbending, beaming, and the photon energy only modify the observed line profiles at a level of a few percent. The shaded region marks the excluded part of the parameter space (see JP11).

In the following, I investigate the prospects of such QPOs to reduce the correlation between the spin and the parameter $\epsilon_{3}$. In this chapter, I focus as a proof of principle on one particular QPO model, the diskoseismology model (see Wagoner 2008 for a review), where QPOs are identified as so-called gravity modes (g-modes; Perez et al. 1997) and corrugation modes (c-modes; Silbergleit et al. 2001). Similar conclusions can be reached for other QPO models, e.g., Kluźniak \& Abramowicz (2001); Abramowicz et al. (2003); Rezolla et al. (2003). I extend my previous analysis of the diskoseismology model in the context of the quasiKerr metric (Johannsen \& Psaltis 2011a) to my new metric, which allows us to 
discuss the dynamical frequencies of accretion disks for arbitrary values of the spin of the black hole.

Here, I will only discuss the fundamental g- and c-modes. For any given values of the spin and the parameter $\epsilon_{3}$, the fundamental g-mode occurs at the radius where the radial epicyclic frequency has a maximum (Perez et al. 1997), while the fundamental c-mode corresponds to the Lense-Thirring frequency evaluated at the ISCO (Silbergleit et al. 2001). I derive expressions for these modes in my metric in Appendix B.

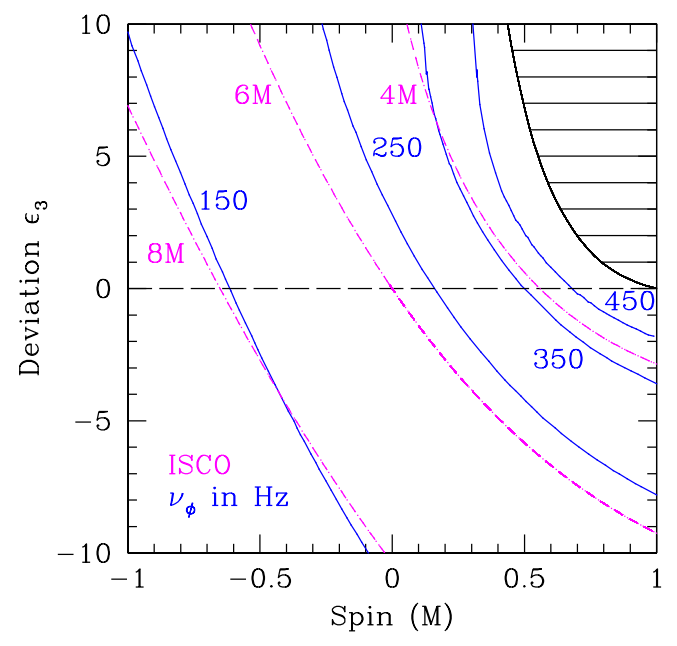

Figure 9.7 Contours of constant Keplerian frequency evaluated at the ISCO for a $10 M_{\odot}$ black hole as a function of the spin and the parameter $\epsilon_{3}$. The Keplerian frequency increases with increasing values of the spin and decreasing values of the parameter $\epsilon_{3}$. For comparison, I also plot contours of constant ISCO radius. The shaded region marks the excluded part of the parameter space (see JP11).

In Figures 9.7 and 9.8, I plot contours of constant Keplerian, g-mode, and c-mode frequencies for a $10 M_{\odot}$ black hole as a function of the spin and the parameter $\epsilon_{3}$. In all three cases, the contours follow curves of decreasing values of 

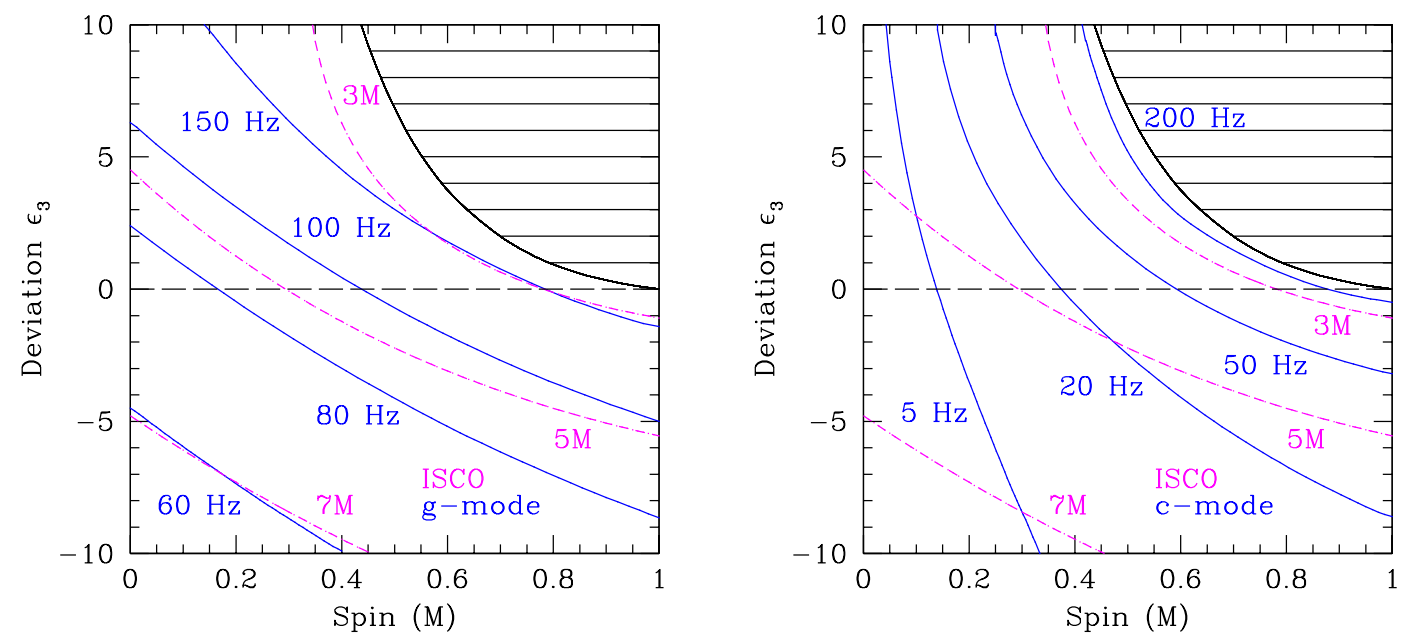

Figure 9.8 Contours of constant (left) g-mode and (right) c-mode frequencies for a $10 M_{\odot}$ black hole as a function of the spin and the parameter $\epsilon_{3}$. Both frequencies increase with increasing values of the spin and decreasing values of the parameter $\epsilon_{3}$. For comparison, I also plot contours of constant ISCO radius. The shaded region marks the excluded part of the parameter space (see JP11).

the parameter $\epsilon_{3}$ for increasing values of the spin. I also plot contours of constant ISCO radius for comparison (see Figure 5 in JP11).

Up to intermediate values of the spin $a \lesssim 0.5 M$, I recover qualitatively the same dependence of these frequencies on the spin and the deviation parameter as I found for the corresponding frequencies in the quasi-Kerr metric (Johannsen \& Psaltis 2011a). Both the Keplerian frequency and the fundamental g-mode vary as a function of the spin and the parameter $\epsilon_{3}$ in a manner that is very similar to the dependence of the ISCO radius on these parameters unless the deviation from the Kerr metric is very large. In contrast, the contours of constant c-mode frequency are a lot steeper in the $\left(a, \epsilon_{3}\right)$-plane than the contours of constant ISCO radius. At large spins, all three frequencies follow contours that nearly align with 
the contours of constant ISCO radius.

As a result, at least within this QPO model, the improvement of a measurement of the spin and the parameter $\epsilon_{3}$ through the combination of spectral and timing techniques will depend on the mode that is identified. For reasonable deviations from the Kerr metric, only low-frequency c-modes (e.g., modes with frequencies below $\sim 10 \mathrm{~Hz}$ for a $10 M_{\odot}$ black hole, see Figure 9.8) can significantly reduce the correlation between the spin and the parameter $\epsilon_{3}$ from an iron line measurement. 


\subsection{Conclusions}

In this chapter, I simulated profiles of fluorescent iron lines for values of the spin $0 \leq a / M \leq 1$ and the parameter $-2 \leq \epsilon_{3} \leq 2$ as long as the spacetime harbors an actual black hole (see JP11). I showed that deviations from the Kerr metric lead to flux shifts that occur primarily at high energies and in the low-energy tail of the line profiles. I demonstrated that these changes can be significant, especially at high spins, and cause a modification of the flux ratio of the two peaks of the line profile. I identified this change as a potential signature of a violation of the no-hair theorem, which, in practice, has to be disentangled from the effect of changing the emissivity index $\alpha$.

I estimated the required precision of future X-ray missions such as Astro- $H$ or ATHENA in order to be able to test the no-hair theorem with fluorescent iron lines for disk inclinations of $\theta_{0}=30^{\circ}$ and $\theta_{0}=60^{\circ}$. As an example, I showed that at an inclination angle of $\theta_{0}=30^{\circ}$, measurements with a precision of $\sim 5 \%$ can constrain the parameter $\left|\epsilon_{3}\right|$ to a value less than unity irrespective of the spin of the black hole.

However, I also showed that line profiles from spacetimes with different spins and values of the parameter $\epsilon_{3}$, but with identical locations of the ISCO, are practically indistinguishable. I showed that other alterations of the line profiles due to modifications of the spacetime only contribute at the percent level. I argued that this correlation can be partially reduced in combination with other observables, which do not depend primarily on the location of the ISCO, such as QPOs.

A prime candidate for a test of the no-hair theorem with fluorescent iron lines is the supermassive black hole in the active galaxy 1H0707-495. Recent observations with XMM-Newton revealed both broad iron $\mathrm{K}$ - and L-line transitions 
(Fabian et al. 2009). Assuming a Kerr black hole, these observations found $a>0.98 M$ at an inclination of $\theta_{0}=55.7^{\circ}$ (Fabian et al. 2009). Due to its high spin, I expect relatively tight constraints on potential deviations from the Kerr metric from this source.

Another promising approach for testing the no-hair theorem lies in observations of fluorescent iron line profiles in the time domain. Localized regions of iron line emission follow nearly Keplerian orbits around the black hole and appear as arcs in the dynamical energy spectra (Brenneman et al. 2009). Upcoming X-ray timing missions, such as the Large Observatory For $x$-ray Timing (LOFT), will for the first time have the sensitivity to perform time-resolved observations of iron lines and trace these hot spots as they orbit around the black hole. I will analyze the prospects of this technique for tests of the no-hair theorem in a forthcoming paper. 


\section{CHAPTER 10}

\section{Orbital Evolution of Black-Hole X-RAY Binaries}

In this chapter, I constrain the asymptotic curvature radius $L$ in the RS2 scenario by considering the evaporation of black holes in X-ray binaries. A mass loss of the black hole in the extra dimension leads to an evolution of the orbit, which is potentially measurable. Competing effects are the orbital period evolution caused by magnetic braking and the evolution of the companion star (see, e.g., Verbunt 1993). The contents of this chapter were published in Johannsen, Psaltis \& McClintock (2009). 


\subsection{Orbital Evolution of a Black-Hole Binary in Braneworld Gravity}

In this section, I derive the rate of change of the orbital period of a binary system that harbors a black hole following closely the works of Will \& Zaglauer (1989) and Psaltis (2007). In my treatment, I also include the effect of CFT emission of the black hole in the extra dimension.

For a black hole of mass $m_{1}$ with a companion star of mass $m_{2}$ on a circular orbit, the rate of change of the orbital angular momentum, $J \equiv \mu \sqrt{G m a}$, is

$$
\begin{gathered}
\frac{\dot{J}}{J}=\frac{1}{J} \frac{\partial J}{\partial m_{1}} \dot{m_{1}}+\frac{1}{J} \frac{\partial J}{\partial m_{2}} \dot{m_{2}}+\frac{1}{J} \frac{\partial J}{\partial a} \dot{a} \\
=\left(1-\frac{1}{2} \frac{m_{1}}{m_{1}+m_{2}}\right) \frac{\dot{m_{1}}}{m_{1}}+\left(1-\frac{1}{2} \frac{m_{2}}{m_{1}+m_{2}}\right) \frac{\dot{m_{2}}}{m_{2}}+\frac{1}{2} \frac{\dot{a}}{a},
\end{gathered}
$$

where $m \equiv m_{1}+m_{2}, \mu \equiv m_{1} m_{2} / m$, and $a$ is the semi-major axis. I set $m_{1}=q m_{2}$ and $\dot{m}_{1}=-\beta \dot{m}_{2}-\dot{M}$, where $\dot{M}$ is the rate of black-hole evaporation into the higher-dimensional bulk. I then obtain

$$
\frac{\dot{J}}{J}=\left(1-\frac{\beta}{q}-\frac{1}{2} \frac{1-\beta}{1+q}\right) \frac{\dot{m}_{2}}{m_{2}}-\left(1+\frac{1}{2} \frac{q}{1+q}\right) \frac{\dot{M}}{m_{1}}+\frac{1}{2} \frac{\dot{a}}{a} .
$$

Angular momentum may be lost because of mass loss from the system or because of the effect of magnetic braking. This leads to

$$
\frac{\dot{J}}{J}=j_{\mathrm{w}}(1-\beta) \frac{1+q}{q} \frac{\dot{m}_{2}}{m_{2}}+\frac{\dot{J}_{\mathrm{mb}}}{J},
$$

where $j_{\mathrm{w}}$ is the specific angular momentum carried away by the stellar wind in units of $2 \pi a^{2} / P, P$ is the orbital period and $\dot{J}_{\mathrm{mb}} / J$ is the rate of angular momentum loss due to magnetic braking. Following Rappaport, Verbunt, \& Joss (1983) I estimate the corresponding torque by the empirical expression

$$
\tau_{\mathrm{mb}} \equiv \dot{J}_{\mathrm{mb}} \simeq-3.8 \times 10^{-30} m_{2} R_{\odot}^{4}\left(\frac{R_{2}}{R_{\odot}}\right)^{\gamma} \omega^{3} \text { dyn cm } .
$$


Here, $\omega$ is the angular frequency of the secondary, $\gamma$ is a parameter that characterizes the strength of the magnetic braking, and $R_{2}$ is the radius of the stellar Roche lobe which is assumed to be filled at all times (Eggleton 1983),

$$
R_{2}=\frac{0.49 q^{-2 / 3}}{0.6 q^{-2 / 3}+\ln \left(1+q^{-1 / 3}\right)} a .
$$

Using the expression for the orbital period

$$
\frac{P}{2 \pi}=\frac{m}{m_{1}^{3} m_{2}^{3}} J^{3} G^{-2}
$$

I can evaluate $\dot{J}_{\mathrm{mb}} / J$ as

$$
\begin{aligned}
& \frac{\dot{J}_{\mathrm{mb}}}{J}=C \frac{G\left(m_{1}+m_{2}\right)^{2}}{m_{1}}\left[\frac{0.49 q^{-2 / 3}}{0.6 q^{-2 / 3}+\ln \left(1+q^{-1 / 3}\right)}\right]^{\gamma} \\
& \times\left[\frac{\sqrt{G\left(m_{1}+m_{2}\right)}}{2 \pi} P\right]^{\frac{2}{3}(\gamma-5)},
\end{aligned}
$$

with

$$
C \equiv-3.8 \times 10^{-30} R_{\odot}^{4-\gamma} .
$$

Additionally, from the period equation (10.6) together with my expressions for $m_{1}$ and $\dot{m}_{1}$ I find

$$
\begin{gathered}
\frac{\dot{P}}{P}=\frac{3}{2} \frac{\dot{a}}{a}-\frac{1}{2} \frac{-\beta \dot{m}_{2}-\dot{M}+\dot{m}_{2}}{m_{1}+m_{2}} \\
=-\frac{1}{2} \frac{1-\beta}{1+q} \frac{\dot{m}_{2}}{m_{2}}+\frac{1}{2} \frac{q}{1+q} \frac{\dot{M}}{m_{1}}+\frac{3}{2} \frac{\dot{a}}{a} .
\end{gathered}
$$

Using

$$
\frac{\dot{q}}{q}=-\frac{\beta+q}{q} \frac{\dot{m_{2}}}{m_{2}}-\frac{\dot{M}}{m_{1}},
$$

I obtain the rate of change of the radius of the companion

$$
\frac{\dot{R}_{2}}{R_{2}}=\frac{\dot{a}}{a}+\frac{2}{3} \frac{\beta+q}{q}\left[1-\frac{0.6+0.5 q^{1 / 3}\left(1+q^{-1 / 3}\right)^{-1}}{0.6+q^{2 / 3} \ln \left(1+q^{-1 / 3}\right)}\right] \frac{\dot{m}_{2}}{m_{2}}
$$




$$
+\frac{2}{3}\left[1-\frac{0.6+0.5 q^{1 / 3}\left(1+q^{-1 / 3}\right)^{-1}}{0.6+q^{2 / 3} \ln \left(1+q^{-1 / 3}\right)}\right] \frac{\dot{M}}{m_{1}} .
$$

The third effect that dictates the change of the orbital period in a binary system is the evolution of the companion star. As the secondary leaves the main sequence and starts to burn helium, it expands rapidly. Following Webbink, Rappaport, \& Savonije (1983) and Verbunt (1993) I estimate the rate of change of the radius of a star leaving the main sequence as

$$
\left(\frac{\dot{R}_{2}}{R_{2}}\right)_{\mathrm{ev}}=\left(c_{1}+2 c_{2} y+3 c_{3} y^{2}\right) \frac{\dot{M}_{\mathrm{c}}}{M_{\mathrm{c}}}
$$

Here, $M_{\mathrm{c}}$ is the core mass of the companion, $y \equiv \ln \left(M_{\mathrm{c}} / 0.25 M_{\odot}\right)$, and $c_{1}, c_{2}$ and $c_{3}$ are constants that depend on the composition of the core. The core mass changes in time according to (Verbunt 1993)

$$
\dot{M}_{\mathrm{c}} \simeq 1.37 \times 10^{-11}\left(\frac{L_{2}}{L_{\odot}}\right) M_{\odot} \mathrm{yr}^{-1} .
$$

In this expression, $L_{2}$ is the luminosity of the companion, which is determined by the core mass (Webbink et al. 1983) according to the empirical relation

$$
\ln \left(\frac{L_{2}}{L_{\odot}}\right)=a_{0}+a_{1} y+a_{2} y^{2}+a_{3} y^{3}
$$

with $a_{0}, a_{1}, a_{2}$, and $a_{3}$ constants depending on the core composition. Combining equations (10.12) - (10.14) leads to

$$
\begin{aligned}
\left(\frac{\dot{R}_{2}}{R_{2}}\right)_{\mathrm{ev}} \simeq & 1.37 \times 10^{-11} \times 4^{a_{1}}\left(c_{1}+2 c_{2} y+3 c_{3} y^{2}\right) \\
& e^{a_{0}+a_{2} y^{2}+a_{3} y^{3}}\left(\frac{M_{\mathrm{c}}}{M_{\odot}}\right)^{a_{1}-1} \mathrm{yr}^{-1} .
\end{aligned}
$$

I now define the adiabatic index for the companion star as

$$
\xi_{\text {ad }} \equiv \frac{d \ln R_{2}}{d \ln m_{2}}
$$


and obtain

$$
\begin{aligned}
\frac{\dot{a}}{a} & =\left[\xi_{\text {ad }}-\frac{2}{3} \frac{\beta+q}{q}\left(1-\frac{0.6+0.5 q^{1 / 3}\left(1+q^{-1 / 3}\right)^{-1}}{0.6+q^{2 / 3} \ln \left(1+q^{-1 / 3}\right)}\right)\right] \frac{\dot{m}_{2}}{m_{2}} \\
& -\frac{2}{3}\left[1-\frac{0.6+0.5 q^{1 / 3}\left(1+q^{-1 / 3}\right)^{-1}}{0.6+q^{2 / 3} \ln \left(1+q^{-1 / 3}\right)}\right] \frac{\dot{M}}{m_{1}}-\left(\frac{\dot{R}_{2}}{R_{2}}\right)_{\mathrm{ev}} .
\end{aligned}
$$

In the following I will estimate the value of $\xi_{\text {ad }}$ from the expressions for the stellar radius $R$ and mass $m_{2}$ given by Kalogera \& Webbink (1996).

Combining the equations I derived above, I obtain the rate of change of the orbital period of the binary

$$
\begin{aligned}
\frac{\dot{P}}{P}= & Q_{0} \frac{\dot{M}}{m_{1}}+Q_{2} \frac{\left(m_{1}+m_{2}\right)^{2}}{m_{1}}\left[\frac{0.49 q^{-2 / 3}}{0.6 q^{-2 / 3}+\ln \left(1+q^{-1 / 3}\right)}\right]^{\gamma} \\
& \times\left[\frac{\sqrt{G\left(m_{1}+m_{2}\right)}}{2 \pi} P\right]^{\frac{2}{3}(\gamma-5)} \\
& +Q_{3}\left(c_{1}+2 c_{2} y+3 c_{3} y^{2}\right) e^{a_{0}+a_{2} y^{2}+a_{3} y^{3}}\left(\frac{M_{\mathrm{c}}}{M_{\odot}}\right)^{a_{1}-1}
\end{aligned}
$$

In this equation I have introduced the quantities

$$
\begin{aligned}
Q_{0} \equiv & \frac{1}{2} \frac{1-\beta}{1+q} \frac{1+\frac{1}{2} \frac{q}{1+q}+\frac{1}{3} \mathcal{A}}{D}+\frac{1}{2} \frac{q}{1+q} \\
& +\frac{3}{2} \frac{\left(\frac{2}{3} \frac{\beta+q}{q} \mathcal{A}-\xi_{\mathrm{ad}}\right)\left(1+\frac{1}{2} \frac{q}{1+q}+\frac{1}{3} \mathcal{A}\right)}{D}-\mathcal{A} \\
Q_{2} \equiv & \frac{C}{D} G\left(\frac{1}{2} \frac{1-\beta}{1+q}+\frac{\beta+q}{q} \mathcal{A}-\frac{3}{2} \xi_{\mathrm{ad}}\right) \\
Q_{3} \equiv & 1.37 \times 10^{-11} \times 4^{a_{1}}\left[\frac{1}{4 D} \frac{1-\beta}{1+q}+\frac{1}{2 D}\left(\frac{\beta+q}{q} \mathcal{A}-\frac{3}{2} \xi_{\mathrm{ad}}\right)-\frac{3}{2}\right] \\
\mathcal{A} \equiv & 1-\frac{0.6+0.5 q^{1 / 3}\left(1+q^{-1 / 3}\right)^{-1}}{0.6+q^{2 / 3} \ln \left(1+q^{-1 / 3}\right)}
\end{aligned}
$$

and

$$
D \equiv j_{\mathrm{w}}(1-\beta) \frac{1+q}{q}-1+\frac{\beta}{q}+\frac{1}{2} \frac{1-\beta}{1+q}-\frac{1}{2}\left(\xi_{\mathrm{ad}}-\frac{2}{3} \frac{\beta+q}{q} \mathcal{A}\right)
$$


For the "evaporation" of the black hole due to the emission of CFT modes, which are the dual description of the infinite dimension, I use (Emparan et al. 2003)

$$
\dot{M}=2.8 \times 10^{-3}\left(\frac{M_{\odot}}{m_{1}}\right)^{2}\left(\frac{L}{1 \mathrm{~mm}}\right)^{2} M_{\odot} \mathrm{yr}^{-1},
$$

where $L$ is the asymptotic AdS radius of curvature. Defining

$$
Q_{1} \equiv 2.8 \times 10^{-3} Q_{0} \mathrm{yr}^{-1}
$$

I arrive at my final equation for the orbital period evolution:

$$
\begin{aligned}
\frac{\dot{P}}{P}= & Q_{1}\left(\frac{M_{\odot}}{m_{1}}\right)^{3}\left(\frac{L}{1 \mathrm{~mm}}\right)^{2}+Q_{2} \frac{\left(m_{1}+m_{2}\right)^{2}}{m_{1}} \\
& \times\left[\frac{0.49 q^{-2 / 3}}{0.6 q^{-2 / 3}+\ln \left(1+q^{-1 / 3}\right)}\right]^{\gamma} \times\left[\frac{\sqrt{G\left(m_{1}+m_{2}\right)}}{2 \pi} P\right]^{\frac{2}{3}(\gamma-5)} \\
& +Q_{3}\left(c_{1}+2 c_{2} y+3 c_{3} y^{2}\right) e^{a_{0}+a_{2} y^{2}+a_{3} y^{3}}\left(\frac{M_{\mathrm{c}}}{M_{\odot}}\right)^{a_{1}-1}
\end{aligned}
$$

For my analysis it is important that the companion star remains in contact with its Roche lobe. Should the black-hole evaporation be so strong that magnetic braking is negligible (as well as stellar evolution), then the Roche lobe of the secondary will grow and the system will eventually get out of contact. This, however, can only occur on timescales of at least $10^{8} \mathrm{yr}$ which is much larger than the observational timescales of interest. Studying other implications of the loss of contact for the evolution of the black-hole binary is beyond the scope of this chapter. 


\subsection{Results}

In this section, I investigate the potential of the currently known black-hole binary systems to constrain the rate of black-hole evaporation into higher dimensions. I will start with a general discussion of the various systems and then focus on the system A0620-00 in particular.

First I investigate which term in equation (10.26) dominates the period evolution for a given period $P$, black hole and companion masses $m_{1}, m_{2}$, respectively, and curvature radius $L$. Figure 1 shows the orbital periods versus the companion masses of the observed systems. On the same graph I plot the curves along which the evaporation term equals the magnetic braking term for $L=0.1 \mu \mathrm{m}, L=1 \mu \mathrm{m}$, $L=10 \mu \mathrm{m}$, and $L=100 \mu \mathrm{m}$. The evaporation dominates above the lines, whereas below the lines the magnetic braking dominates. Recent numerical simulations (Yungelson \& Lasota 2008) showed that the expression for magnetic braking is actually overestimated for low-mass black-hole binaries, which further increases the predominance of the evaporation term. The parameters I used in this figure are $\xi_{\text {ad }}=0.8, \beta=0, j_{\mathrm{w}}=0$, and $\gamma=0$, and I set the black-hole mass to a nominal value of $10 M_{\odot}$.

For companion masses $\gtrsim 1 M_{\odot}$, there exists a maximum period beyond which systems contain companion stars that have evolved past the base of the giant branch (see the curve marked BGB in Figure 1). For these systems, the evolution of the companion star completely dominates the rate of change of the orbital period for any plausible value of the asymptotic curvature radius $L$.

I find that two sources have relatively large orbital periods and are probably evolved, while the other systems group around the line that corresponds to $L=$ $1 \mu \mathrm{m}$ and between the lines corresponding to $L=10 \mu \mathrm{m}$ and $L=100 \mu \mathrm{m}$. Among 
the latter I identify the system A0620-00 as a promising candidate both because of the theoretical expectation shown in this figure and because of the extensive historical monitoring of its orbital period. I analyze this source more closely in the following.

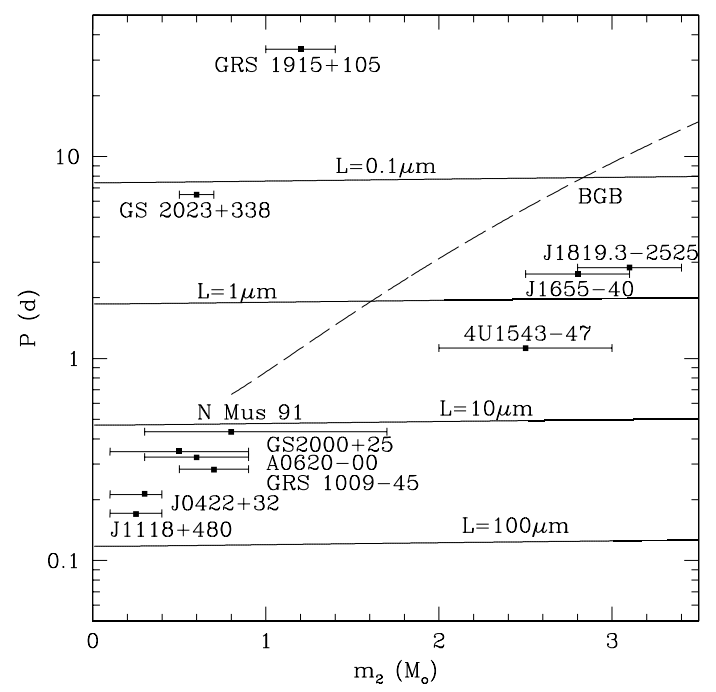

Figure 10.1 The orbital period $P$ of observed black-hole binary systems versus the mass $m_{2}$ of the companion star. Four separatrices are shown for different values of the asymptotic AdS curvature radius $L$ for a nominal black-hole mass of $10 M_{\odot}$. Below the lines, magnetic braking dominates. Above the lines, black-hole evaporation dominates. Binaries above the curve marked BGB contain companions beyond the base of the giant branch; for these systems, the evolution of the companion completely dominates the orbital evolution of the binary. The parameters for this graph are $\xi_{\mathrm{ad}}=0.8, \beta=0, j_{\mathrm{w}}=0$, and $\gamma=0$. Note that the separatrices only depend weakly on $m_{2}$ as long as $\gamma=0$.

The short-period (0.32 d) binary A0620-00 has a secondary that resembles a main sequence star, as indicated by its spectrum and by its chemical abundances (González Hernández et al. 2004). Its mass and radius are also comparable to that of a main-sequence K4 star (Marsh et al. 1994). In particular, the mean density 


\begin{tabular}{lccc}
\multicolumn{4}{c}{ Some Observed Properties of X-Ray Binaries } \\
\hline \hline X-Ray Binary & $P(h)$ & $q$ & $m_{1}\left(M_{\odot}\right)$ \\
\hline GRS1915+105 & 816 & 12 & $14 \pm 4$ \\
J1118+480 & 4.1 & $\sim 20$ & $6.8 \pm 0.4$ \\
GS2023+338 & 155.3 & $17 \pm 1$ & $12 \pm 2$ \\
GS2000+25 & 8.3 & $24 \pm 10$ & $10 \pm 4$ \\
H1705-25 & 12.5 & $>19$ & $6 \pm 2$ \\
GRS1009-45 & 6.8 & $7 \pm 1$ & $5.2 \pm 0.6$ \\
N Mus 91 & 10.4 & $6.8 \pm 2$ & $6_{-2}^{+5}$ \\
A0620-00 & 7.8 & $17 \pm 1$ & $10 \pm 5$ \\
J0422+32 & 5.1 & $9.0_{-2.7}^{+2.2}$ & $4 \pm 1$ \\
J1819.3-2525 & 67.6 & $2.31 \pm 0.08$ & $7.1 \pm 0.3$ \\
J1655-40 & 62.9 & $2.39 \pm 0.15$ & $6.6 \pm 0.5$ \\
4U1543-47 & 27.0 & $3.6 \pm 0.4$ & $9.4 \pm 1$ \\
\hline
\end{tabular}

Table 10.1 Orbital periods, mass ratios, and black hole masses of observed X-ray binaries. Most data compiled by Charles \& Coe (2006); mass ratio of A0620-00 taken from Neilsen, Steeghs \& Vrtilek (2008).

of this Roche-lobe-filling secondary, which is precisely determined by its orbital period (Frank et al. 2002), is only $\sim 25 \%$ below that of a normal K4 dwarf. However, the secondary of A0620-00 is not a normal star, given the extraordinary evolutionary history of this black-hole binary system (e.g., de Kool et al. 1986). Nevertheless, for my purposes the secondary functions like a main-sequence star: it is not evolving on a nuclear time scale and the system is kept in contact by magnetic braking (Justham et al. 2006).

Thus I can neglect the evolution term in equation (10.26) and plot the expected rate of change of its orbital period $P$ as a function of the asymptotic AdS curva- 
ture radius $L$ (see Figure 2). The parameters for this plot are $\beta=0, j_{\mathrm{w}}=0$, $\gamma=0$, and $\xi_{\mathrm{ad}}=0.8$. I see that for $L \lesssim 20 \mu \mathrm{m}$ the magnetic braking dominates and the rate of orbital period change is constant because it is independent of $L$. For $L \gtrsim 20 \mu \mathrm{m}$ the black-hole evaporation dominates and the orbital period derivative increases with increasing AdS curvature as expected. This shows that A0620-00 theoretically allows for a constraint on $L$ as low as $20 \mu \mathrm{m}$, assuming that $m_{1}=10 M_{\odot}$. Since $m_{1}$ has only been measured to an accuracy of $\pm 50 \%$, the constraint can even be reduced to a few microns. I will return to the question of the black-hole mass in the next section.

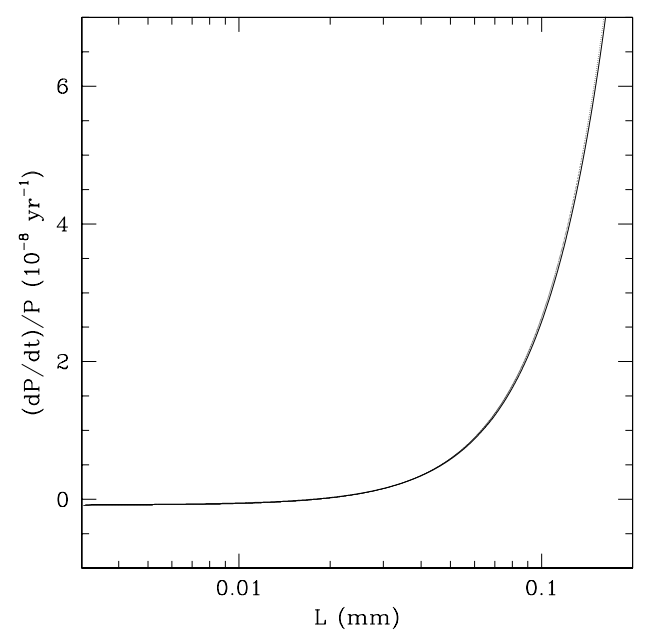

Figure 10.2 The rate of change of the orbital period $P$ of the binary system A0620-00 versus the asymptotic curvature radius $L$ in the extra dimension. The parameters are $\xi_{\text {ad }}=0.8, \beta=0, j_{\mathrm{w}}=0$, and $\gamma=0$. The transition from predominant magnetic braking (constant negative rate) to predominant black-hole evaporation (positive and rapidly increasing rate) occurs at $L \simeq 20 \mu \mathrm{m}$.

In order to determine the dependence of the orbital period evolution on the parameters $j_{\mathrm{w}}, \beta$, and $\gamma$, I plot the rate of change of the orbital period as a function 
of one parameter while holding the others constant. In all plots, I set $\xi_{\text {ad }}=0.8$ (estimated from Kalogera \& Webbink 1996) for the companion mass in this system and evaluate the period evolution rate at the current experimental upper limit of the AdS curvature of $L=44 \mu \mathrm{m}$ (Kapner et al. 2007). Figure 3 shows the dependence of the rate of change of the orbital period on the parameters $j_{\mathrm{w}}, \beta$, and $\gamma$, respectively. First I note that for large values of the parameters the period increases. This behavior is entirely due to the high mass ratio $q=m_{1} / m_{2}$ measured for this source; for other sources with substantially smaller mass ratios, the behavior is not monotonic. Furthermore, I find that the rate of change of the orbital period is the smallest when $j_{\mathrm{w}}=0$ (no angular momentum loss due to stellar wind), $\beta=0$ (no accretion), and $\gamma=0$. I choose these values for the respective parameters in the following discussion, where I are aiming to calculate a lower limit on the expected rate of change of the orbital period.
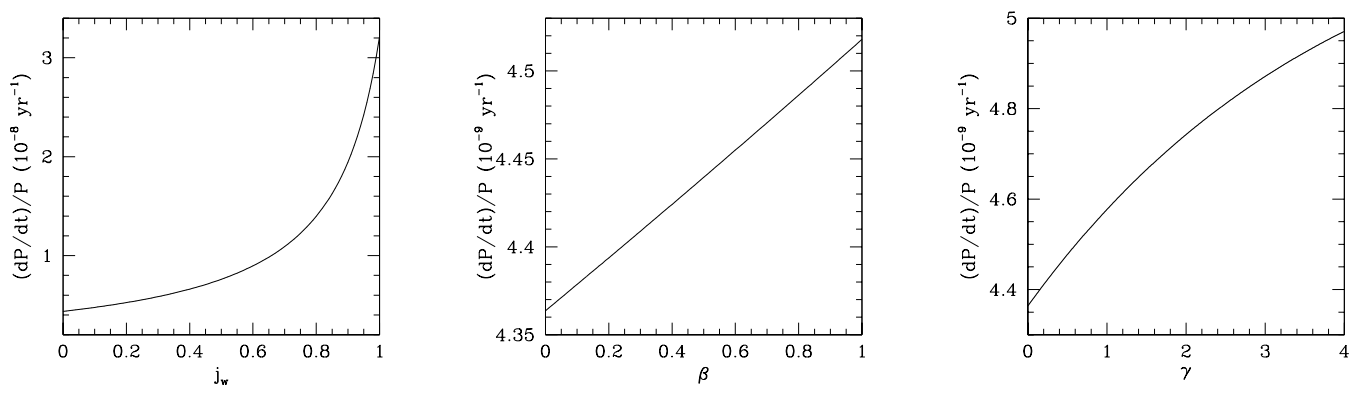

Figure 10.3 The rate of change of the orbital period $P$ (in years) of the binary system A0620-00 versus the specific angular momentum removed by the wind $j_{\mathrm{w}}$, the accretion parameter $\beta$, and the magnetic braking parameter $\gamma$, for $L=$ $44 \mu \mathrm{m}$ and $\xi_{\mathrm{ad}}=0.8$. On varying one parameter, the others are held constant at the respective values $j_{\mathrm{w}}=0, \beta=0$, and $\gamma=0$. 


\section{CHAPTER 11}

\section{CONSTRAINTS ON THE SIZE OF EXTRA DIMENSIONS}

In this chapter, I use previously published measurements of the orbital period of A0620-00 and XTE J1118+480 to set an upper limit on the size $L$ of the asymptotic curvature in the extra dimension. The contents of this chapter were published in Johannsen, Psaltis \& McClintock (2009) and Johannsen (2009). 


\section{$11.1 \quad \mathrm{~A} 0620-00$}

The orbital period of A0620-00 $(P=0.32 \mathrm{~d})$ has been measured several times during the past two decades. A convenient orbital phase reference is the time of maximum radial velocity $T_{0}$. Four measured values of $T_{0}$, which span 22 years, are given in Table 2. These times and the individual determinations of the orbital period $P$ uniquely determine the cycle number $n$. The table also gives the calculated times of maximum velocity based on a simple ephemeris with a constant orbital period and referenced to the most precise and recent determination of $T_{0}$ (see footnote $a$ of Table 2). The differences between the observed and calculated times are smaller than their corresponding uncertainties, and thus there is no evidence for any change in the orbital period during the past 22 years. Note that with modern telescopes and instrumentation one can routinely achieve a precision of several seconds in $T_{0}$ (see the last entry in the table) in $\sim 10$ hours of radial velocity observations of a source like A0620-00 (Neilsen et al. 2008).

Of interest to us is a secure limit on the rate of change of the orbital period. A constant rate of change of the period will result in a quadratic variation in $T_{0}$ (e.g., Kelley et al. 1983). The time of the $n$th value of $T_{0}$ is then given by

$$
t_{\mathrm{n}}=t_{0}+P n+\frac{1}{2} P \dot{P} n^{2},
$$

where $P$ and $\dot{P}$ are the orbital period and its derivative, respectively, at time $t_{0} ; n$ is the orbital cycle number. Following the standard procedure and using the IDL routine curvefit, I fitted for the three parameters $t_{0}, P$ and $\dot{P}$ using the four observed values of $T_{0}$ given in Table 11.1 (Figure 11.1). The fit yields $\dot{P}=(-1.66 \pm 2.64) \times 10^{-11} \mathrm{~s} / \mathrm{s}$. Thus, using a $3 \sigma$-upper limit, the period deriva- 
tive is constrained within the interval $-9.58 \times 10^{-11} \mathrm{~s} / \mathrm{s}<\dot{P}<6.26 \times 10^{-11} \mathrm{~s} / \mathrm{s}$. Next I plot the rate of change of the orbital period versus the AdS curvature for the values of the parameters $\beta, j_{\mathrm{w}}$, and $\gamma$ that lead to the lowest limit of the rate of change of the orbital period for a given value of the asymptotic curvature radius $L$ (Figure 11.2). For $L \gtrsim 20 \mu \mathrm{m}$, the rate of change of the orbital period is smallest for the set of parameters $j_{\mathrm{w}}=0, \beta=0$, and $\gamma=0$, while for $L \lesssim 20 \mu \mathrm{m}$, the parameters $j_{\mathrm{w}}=1, \beta=0$, and $\gamma=0$ minimize the orbital period evolution. In a narrow intermediate region, the corresponding set of parameters is $\beta=1$, and $\gamma=0$, while $j_{\mathrm{w}}$ is arbitrary. Since the measured upper limit on the period derivative in equation (11.1) marks the largest time change of the orbital period, the intersection point of this line with the graph of the smallest rate of change of the orbital period places an upper limit on the asymptotic AdS curvature radius of $L \leq 161 \mu \mathrm{m}$ (Figure 11.2).

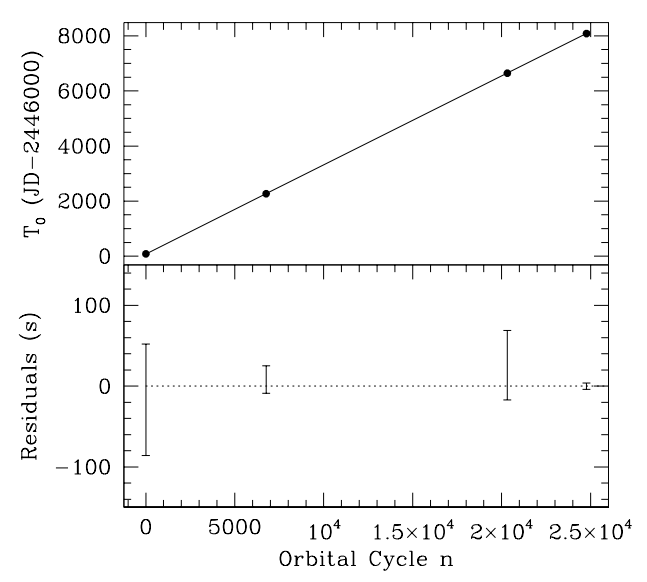

Figure 11.1 The time of maximum radial velocity $T_{0}$ and residuals versus the orbital cycle number $n$ for the binary A0620-00. 
Since the upper limit on the asymptotic curvature radius depends strongly on the mass of the black hole, which has only been measured to an accuracy of $\pm 50 \%$, I plot in Figure 11.3 the upper limit $L_{\max }$ versus the black-hole mass for different limits on the orbital period change. For the upper curve I used the current limit on the orbital period evolution from the fit using equation (11.1), whereas for the lower curve I used a value that is a factor of 10 lower. I see that even the current table-top limit of the asymptotic AdS curvature radius $L=44 \mu \mathrm{m}$ can be improved if the upper limit of the rate of change of the orbital period can be reduced to $10 \%$ of the current value and if the black-hole mass is smaller than $9 M_{\odot}$. In Figure 11.4 I plot the smallest rate of change of the orbital period for black-hole masses (from left to right) $m_{1}=5,10$, and $15 M_{\odot}$, respectively. The parameters for each curve are chosen as in Figure 11.2.

There are ample opportunities to substantially improve the above limit on the asymptotic curvature radius $L$ using my method. For example, because the uncertainty in the orbital period derivative depends quadratically on the orbital cycle number $n$, even a single future observation of the binary A0620-00 that extends the 22 -year baseline by just 5 years (i.e., $n=30423$ ) with a precision of $4 \mathrm{~s}$ would reduce the error in the orbital period derivative $\dot{P}$ by a factor of six. Furthermore, independent limits of comparable quality on $\dot{P}$ and $L$ could be obtained by monitoring the ephemerides of several other black-hole X-ray binary systems (e.g., GRS 1124-683, XTE J1118+480, and 4U 1543-47; Remillard \& McClintock 2006). 
Observed and Computed Times of Maximum Velocity for A0620-00

\begin{tabular}{cccc}
\hline \hline $\begin{array}{c}\text { Orbital Cycle } \\
n\end{array}$ & $\begin{array}{c}T_{0} \text { in JD } \\
\text { observed }\end{array}$ & $\begin{array}{c}T_{0}(n) \text { in JD } \\
\text { computed }\end{array}$ & $\begin{array}{c}T_{0}-T_{0}(n) \\
(\mathrm{s})\end{array}$ \\
\hline 0 & $2,446,082.7481 \pm 0.0008$ & $2,446,082.7483$ & $-17 \pm 69$ \\
6764 & $2,448,267.6155 \pm 0.0002^{(a)}$ & $2,448,267.6154$ & $8 \pm 17$ \\
20321 & $2,452,646.7173 \pm 0.0005$ & $2,452,646.7170$ & $26 \pm 43$ \\
24773 & $2,454,084.77560 \pm 0.00005$ & $2,454,084.77560$ & $0 \pm 4$ \\
\hline
\end{tabular}

Table 11.1 Observed and Computed Heliocentric Times of Maximum Velocity for A0620-00. $T_{0}(n)=$ JD 2,454,084.77560 $-(24773-n) \times 0.32301406$. References: $(1)$ McClintock \& Remillard 1986; (2) Orosz et al. 1994; (3) Shahbaz et al. 2004; (4) Neilsen et al. 2007. ${ }^{(a)} T_{0}$ corrected to date of observation on 1991 January 11 using ephemeris in reference (1).

\subsection{XTE J1118+480}

In order to obtain a constraint on the asymptotic curvature radius $L$ of the extra dimension, it is essential to select a black-hole binary with an unevolved companion star. In that case, the evolution term in equation (10.26) can be neglected, and the binary can be used to constrain the AdS radius as long as the magnetic braking term is negligible compared to the evaporation term. In the following I argue that this approach can be applied to the black-hole binary J1118+480, and I use previous measurements of its orbital period to place a bound on the rate of change of its orbital period.

The system J1118+480 has been monitored for more than a decade (Remillard \& McClintock 2006). The companion star of the black hole resembles a late-type main-sequence star of spectral type K7 V - M0 V (Wagner et al. 2001). In addition, 


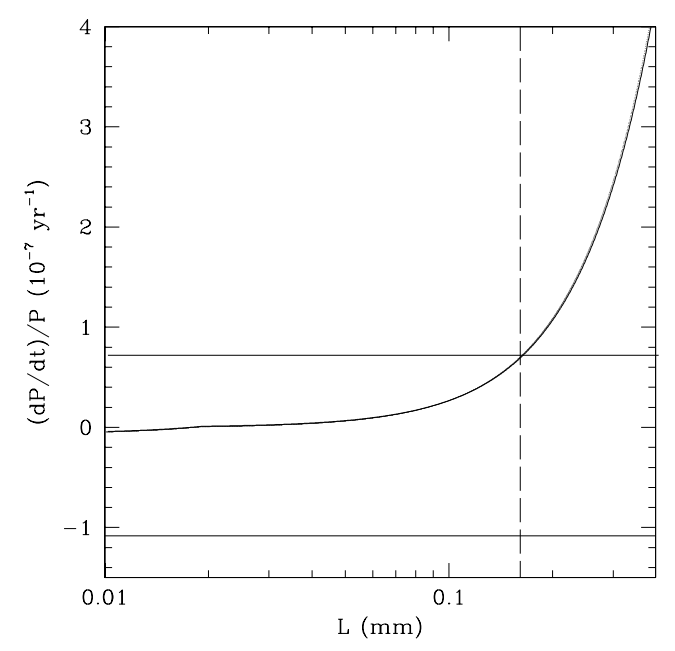

Figure 11.2 The rate of change of the orbital period $P$ of the binary system A0620-00 as a function of the asymptotic AdS curvature radius $L$ for a black-hole mass of $10 M_{\odot}$. For positive values of the period derivative, this line represents the lower limit among all possible values of the period evolution. The $3 \sigma$-error bars of the observed orbital period derivative are shown as horizontal lines. The intersection point of the upper limit on $\dot{P} / P$ with the lower limit curve marks my constraint on the asymptotic curvature in the bulk of $L=161 \mu \mathrm{m}$ (vertical line).

the mean density is only $\sim 50 \%$ higher than for a usual main-sequence M0 star (McClintock et al. 2001), and the mass is only $\sim 50 \%$ lower than that of such a star (see Charles \& Coe 2006).

It is important to note that this is not simply a normal star. It has emerged out of an exeptional evolutionary history (see de Kool et al. 1986 for an example), and it does not evolve on a nuclear timescale. For my analysis, however, it is sufficient that the secondary only behaves like a main-sequence star. Then the evolution term in equation (10.26) is negligible.

Considering only the evaporation term and the magnetic braking term in 


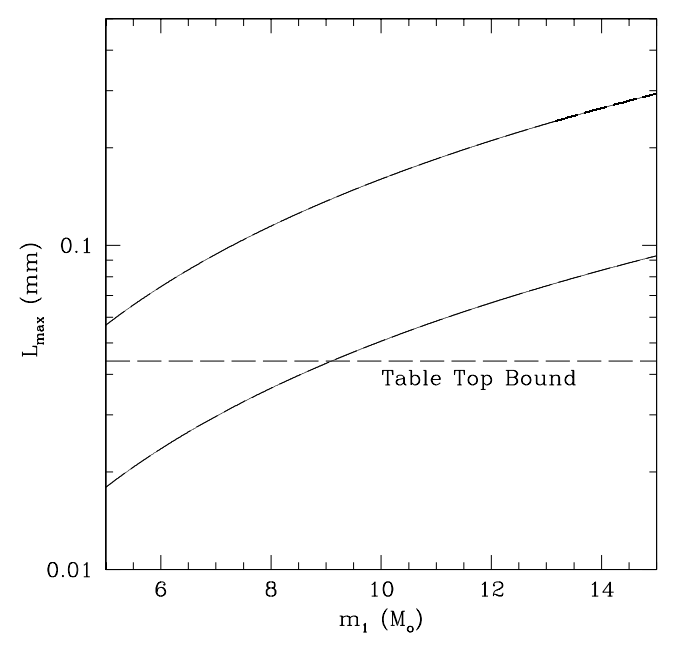

Figure 11.3 The upper limit on the asymptotic AdS curvature radius $L$ as a function of the black hole mass $m_{1}$ of A0620-00 using the current lower limit on the orbital period evolution (upper curve) and on 10\% of that limit (lower curve). The dashed line shows the current upper limit on $L$ from table-top experiments.

equation (10.26), I plot in Figure 11.5 the rate of change of the orbital period versus the asymptotic curvature radius $L$ in the extra dimension for the binary systems J1118+480 and A0620-00. The parameters used in this plot are $\xi_{\text {ad }}=0.8$, $\beta=0, j_{\mathrm{w}}=0$, and $\gamma=0$. For values of the asymptotic curvature radius greater than $L \simeq 35 \mu \mathrm{m}$ the evaporation term dominates the evolution of the orbital period in the case of $\mathrm{J} 1118+480$. Below that value, the magnetic braking is predominant. For the binary A0620-00 the transition occurs at $L \simeq 20 \mu \mathrm{m}$ (Johannsen et al. 2009). Consequently, these sources are similar in constraining the asymptotic curvature radius of the extra dimension.

A measurement of a positive rate of change of the orbital period automatically constrains the asymptotic curvature radius to an interval, as can be seen by 


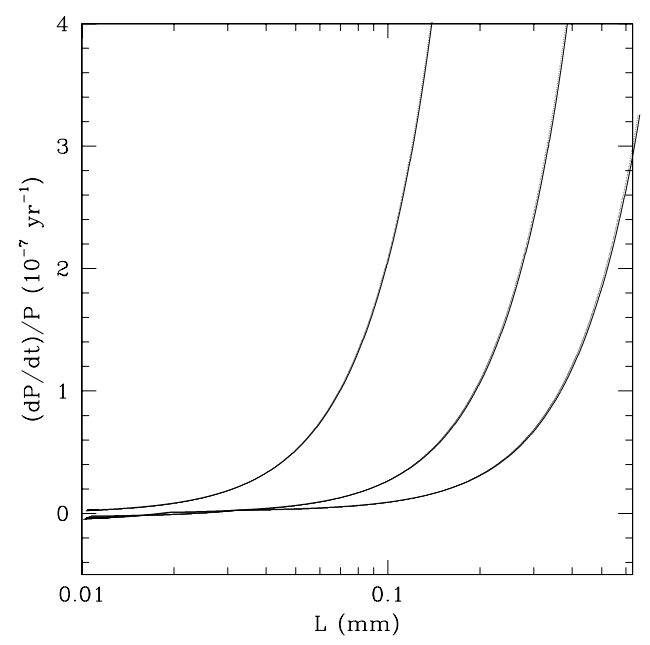

Figure 11.4 The minimum rate of change of the orbital period of the binary A0620-00 versus the asymptotic AdS curvature radius $L$ for (from left to right) $m_{1}=5,10,15 M_{\odot}$, respectively.

simply evaluating the magnetic braking term. For a binary system with a high mass ratio, i.e. $m_{1} / m_{2}>5.5$, the effect of magnetic braking can only decrease the orbital period (Johannsen et al. 2009). Thus such a measurement actually determines the AdS radius $L$ and would prove the existence of an extra dimension.

In Figure 11.2, I plot the rate of change of the orbital period of J1118+480 as a function of the parameters $j_{\mathrm{w}}, \beta$, and $\gamma$. On varying one of them, the others are held constant at the respective values $\beta=0, j_{\mathrm{w}}=0$, and $\gamma=0$. For all plots I choose $\xi_{\text {ad }}=0.8$ and $L=44 \mu \mathrm{m}$, which is the value of the current experimental limit (Kapner et al. 2007). I am interested in the smallest rate of orbital period evolution, so in the following I set the parameters to the respective values $j_{\mathrm{w}}=0$ (no angular momentum loss due to stellar wind), $\beta=0$ (no accretion onto the black hole), and $\gamma=0$. 


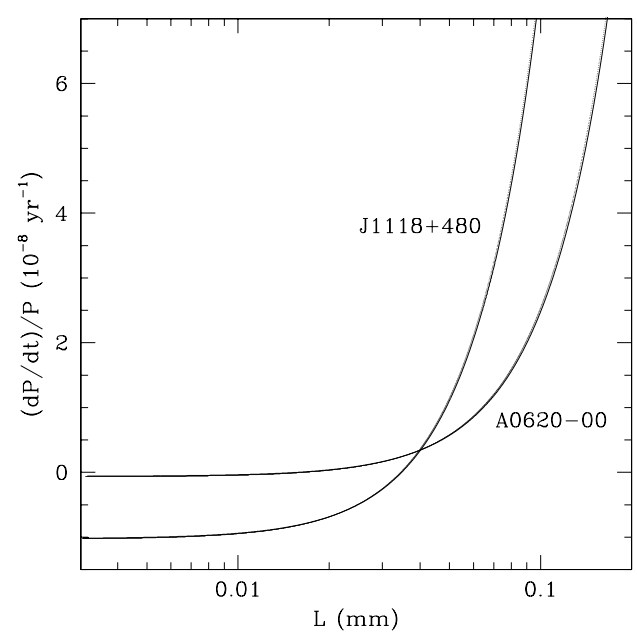

Figure 11.5 The rate of change of the orbital period $P$ of the binary systems $\mathrm{J} 1118+480$ and A0620-00 versus the asymptotic curvature radius $L$ in the extra dimension. The parameters are $\xi_{\text {ad }}=0.8, \beta=0, j_{\mathrm{w}}=0$, and $\gamma=0$. The transition from predominant magnetic braking (constant rate) to predominant blackhole evaporation (rapidly increasing rate) occurs at $L \simeq 20 \mu \mathrm{m}$ (A0620-00) and $L \simeq 35 \mu \mathrm{m}(\mathrm{J} 1118+480)$, respectively.

The orbital period $P$ of $\mathrm{J} 1118+480$ has been measured several times over the past decade. Based on those measurements, I calculate the rate of change of the orbital period. Table 11.2 shows the orbital period measurements for J1118+480. The values are for the most part consistent with each other, indicating an at most small change of the period over the last years.

In order to calculate the rate of change of the orbital period, I fitted the times $T_{0}$ in Table 1 according to equation (11.1) using the IDL routine curvefit. The value from reference (2) was omitted, and instead the more precise reference (3) was used. The fit yields $\dot{P}=(0.7 \pm 3.1) \times 10^{-9} \mathrm{~s} / \mathrm{s}$ which is still consistent with zero quoting $3 \sigma$-errors. The fit and residuals are shown in Figure 11.7. 

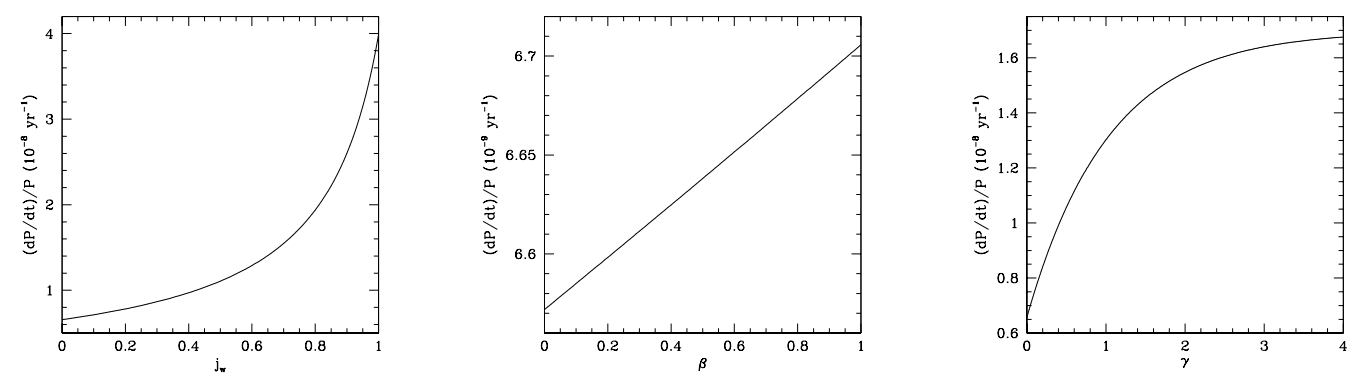

Figure 11.6 The rate of change of the orbital period $P$ (in years) of the binary system J1118+480 versus the specific angular momentum removed by the stellar wind $j_{\mathrm{w}}$, the accretion parameter $\beta$, and the magnetic braking parameter $\gamma$, for $L=44 \mu \mathrm{m}$ and $\xi_{\text {ad }}=0.8$. On varying one parameter, the others are held constant at the respective values $j_{\mathrm{w}}=0, \beta=0$, and $\gamma=0$.

\subsection{Results}

Using the values of the orbital period derivative calculated in $\S 3$, I compute a limit on the asymptotic curvature radius of the extra dimension. For the mass of the black hole I use the best fit value of $m_{1}=6.8 M_{\odot}$ (c.f. Charles \& Coe 2006).

The $3 \sigma$-upper limit on the observed rate of change of the orbital period and the smallest theoretical expectation thereof determine an upper bound on the AdS curvature radius $L$. In Figure 11.8, I plot the rate of change of the orbital period of J1118+480 for the set of parameters that minimizes it (c.f. $\S 3$ ). The intersection point of this curve with the $3 \sigma$-upper limit on the orbital period evolution yields a constraint on the AdS curvature radius of $L<0.97 \mathrm{~mm}$.

This value is comparable to the constraint obtained from A0620-00 (Johannsen et al. 2009). A refinement of that upper bound would be easy to obtain and simply requires an additional measurement of the orbital period. The fact that the period change due to magnetic braking is so large for $\mathrm{J} 1118+480$ in the case where 
Currently Observed Orbital Periods and Times of Measurement for J1118+480

\begin{tabular}{lccc}
\hline \hline$P(d)$ & $T_{0}$ (inf. conj.) in HJD & Orbital Cycle $n$ & Reference \\
\hline $0.169930 \pm 0.000004$ & $2,451,868.8916 \pm 0.0004$ & 0 & 1 \\
$0.17013 \pm 0.00010$ & $2,451,880.1485 \pm 0.0010^{\mathrm{a}}$ & 66 & 2 \\
$0.1699339 \pm 0.0000002$ & $2,451,880.1086 \pm 0.0004$ & 66 & 3 \\
$0.169937 \pm 0.000001$ & $2,452,022.5122 \pm 0.0004$ & 904 & 4 \\
$0.16995 \pm 0.00012$ & $2,453,049.93346 \pm 0.00007$ & 6950 & 5 \\
\hline
\end{tabular}

Table 11.2 The Observed Orbital Periods and Times of Measurement for J1118+480. References: (1) Wagner et al. 2001; (2) McClintock et al. 2001; (3) Torres et al. 2004; (4) Zurita et al. 2002; (5) González Hernández et al. 2008. ${ }^{b}$ Time of maximum velocity.

the black-hole evaporation is negligible $\left(\dot{P} / P \sim-10^{-8} \mathrm{yr}^{-1}\right)$ has two important consequences. First, even one additional measurement of the orbital period in $2009(n=17,000)$ with an error in the ephemeris $T_{0}$ of the order of $0.001 \mathrm{~d}$ would prove the period derivative to be negative at $3 \sigma$, hence measuring the first orbital period evolution of a black-hole X-ray binary. Second, such a measurement would show that black-hole evaporation is negligible against magnetic braking in this binary, which would in turn impose a constraint of the order of $35 \mu \mathrm{m}$ on the size of one extra dimension, the tightest constraint to date.

The constraint on the asymptotic curvature radius also depends on the mass of the primary, but since the black-hole mass has been measured quite precisely $\left(m_{1}=6.8 \pm 0.4 M_{\odot} ;\right.$ from Charles \& Coe 2006), its effect is small. 


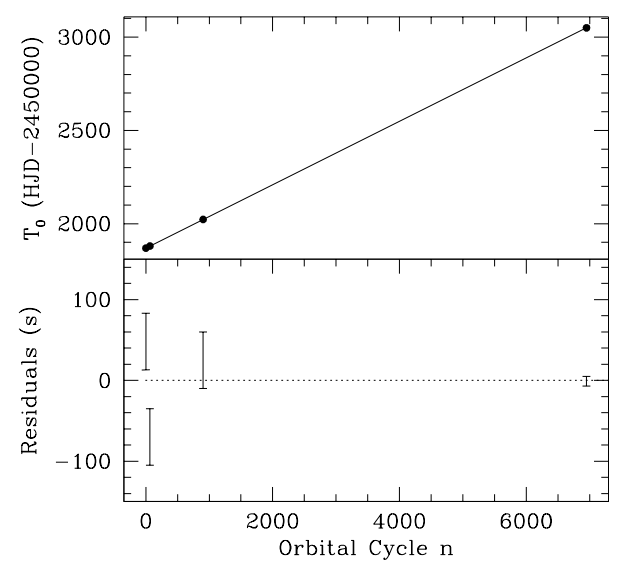

Figure 11.7 The times $T_{0}$ of the inferior conjunction and residuals versus the orbital cycle number $n$ for the black-hole binary J1118 +480 .

\subsection{Discussion}

For a binary system consisting of a black hole and a companion star I derived the rate of change of the orbital period in the RS2 braneworld gravity model incorporating the emission of CFT modes in the extra dimension. Magnetic braking and the evolution of the companion star can also change the orbital period, but they are negligible if the secondary is a main-sequence star and if the asymptotic AdS curvature radius $L$ is large enough so that the evaporation dominates. Measuring the rate of change of the orbital period then allows us to constrain the asymptotic AdS curvature radius.

I analyzed in detail the binary system A0620-00 which is a good candidate for such a constraint for both theoretical and observational reasons. The evaporation term dominates the change of the orbital period as long as the asymptotic curva- 


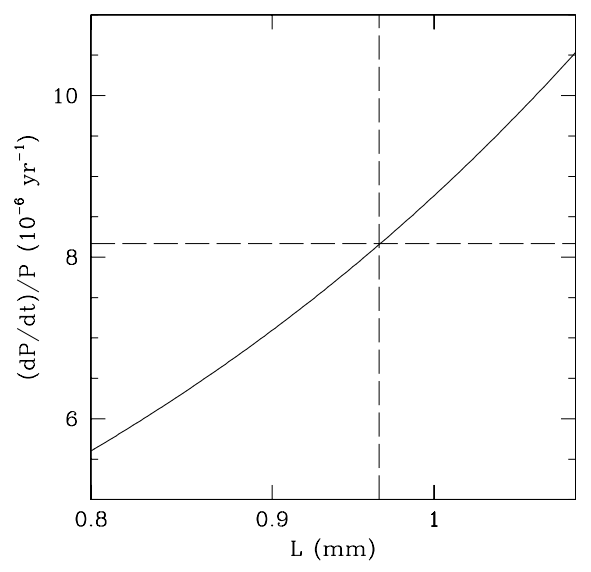

Figure 11.8 The minimum rate of change of the orbital period $P$ of the binary $\mathrm{J} 1118+480$ versus the asymptotic curvature radius $L$ in the extra dimension. The intersection point of the observed $3 \sigma$-upper limit on $\dot{P} / P$ (horizontal line) with the minimum rate of change of the orbital period marks the constraint on the AdS curvature radius of $L<0.97 \mathrm{~mm}$ (vertical line).

ture radius is at least $\sim 20$ microns large. Measurements of the orbital period over the last 20 years allow for a constraint of $L<161 \mu \mathrm{m}$ assuming a black-hole mass of $10 M_{\odot}$. Refining the measurement of the mass of the black hole and of the rate of change of the orbital period can further improve the constraint on the asymptotic curvature radius. As an example I showed that improving the measurement of the rate of change of the orbital period by one order of magnitude will constrain the AdS curvature radius to a value smaller that the current experimental limit $L=44 \mu \mathrm{m}$ (Kapner et al. 2007), provided the black-hole mass is measured not to exceed $9 M_{\odot}$.

The black-hole binary XTE J1118+480 is well-suited for a constraint on the asymptotic curvature radius of the extra dimension in Randall-Sundrum type 
braneworld gravity models. An upper limit on the rate of change of the orbital period of XTE J1118+480 is calculated based on previous measurements of the orbital period from the literature. The lack of observed orbital evolution of this binary imposes a constraint on the asymptotic curvature radius of $L<0.97 \mathrm{~mm}$. This constraint can be significantly improved by only one additional measurement of the orbital period of XTE J1118+480. This would be the first detection of orbital evolution in a black-hole binary. The expected predominance of magnetic braking would provide the best constraint to date on the asymptotic curvature radius of the extra dimension of the order of $35 \mu \mathrm{m}$. This measurement was obtained recently by Gonzáles Hernández et al. (2012).

Considering the other known black-hole X-ray binaries, I see from Figure 10.1 that there are more systems which I can use in constraining the asymptotic curvature radius in the extra dimension. The requirement of unevolved secondaries rules out some of them, but several sources have the potential of a constraint on the curvature radius down to a few microns.

A very exciting aspect of my method is that it not only allows us to constrain the AdS radius $L$, but also to potentially measure it - thereby giving evidence for new physics beyond general relativity and the standard model — provided that the rate of change of the orbital period is measured to be positive. This is due to the fact that, for a black-hole binary with a sufficiently high mass ratio ( $\left.q=m_{1} / m_{2}\right)$, magnetic braking can only shorten the orbital period. The sign of the magnetic braking term in equation (10.26) and hence whether this effect leads to a positive and a negative change of the orbital period is determined exclusively by the prefactor $Q_{2}$. This factor depends only on the mass ratio $q$ and on the parameters $\beta$ and $j_{\mathrm{w}}$ assuming a fixed adiabatic index. In Figure 11.9 I plot the 


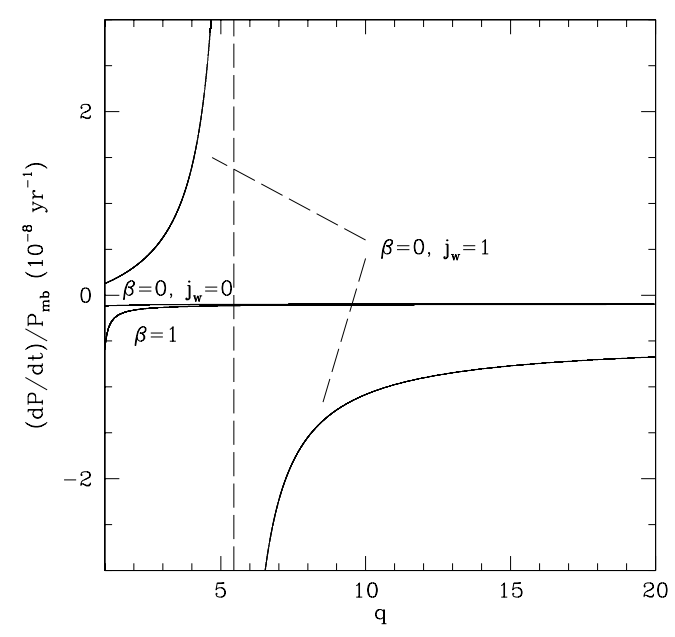

Figure 11.9 The rate of change of the orbital period of the binary A0620-00 due to magnetic braking versus the mass ratio $q=m_{1} / m_{2}$ for several combinations of the parameters $\beta$ and $j_{\mathrm{w}}$. I set $m_{1}=10 M_{\odot}, P=7.8 \mathrm{hr}, \xi_{\text {ad }}=0.8$, and $\gamma=$ 0 . For large values of the mass ratio, varying the parameters only changes the magnitude of the magnetic braking term but not its overall sign.

magnetic braking term for various combinations of the parameters $\beta$ and $j_{\mathrm{w}}$, and I set the black-hole mass and the orbital period to the nominal values $m_{1}=10 M_{\odot}$ and $P=7.8 \mathrm{hr}$, respectively, as well as $\gamma=0$. The mass of the primary, the orbital period and the parameter $\gamma$ only effect the magnitude of the magnetic braking term but not its sign. For all curves I set $\xi_{\text {ad }}=0.8$. I see that the magnetic braking term is negative for $q>5.5$ for any value of the parameters $\beta, j_{\mathrm{w}}$, and $\gamma$.

Thus I conclude that any measurement of a positive orbital period derivative in a black-hole X-ray binary with a mass ratio $q>5.5$ (assuming an unevolved companion star) is strong evidence for new gravitational physics and directly measures the asymptotic curvature radius $L$. Since my method is modeldependent (RS2), such a measurement, together with the table-top experiments, 
would even allow for a distinction between the ADD and the RS2 scenario. Current table-top experiments, that probe Newton's inverse square law in the sub$\mathrm{mm}$ range, are insensitive to the underlying model, so that their results together with a measurement as discussed above would indeed allow for a distinction between ADD and RS2. 


\section{CHAPTER 12}

\section{SUMMARY AND OUTLOOK}

Black holes are among the most fascinating objects in the universe surrounded by matter under extreme conditions in a regime of strong spacetime curvature. The study of these objects will lead to a deeper understanding of their nature, their environment, and their implications for fundamental theories such as general relativity and string theory. At the same time, the wealth and precision of observations across the electromagnetic spectrum from both ground-based and space observatories is opening up ever increasing possibilities to test fundamental properties of black holes. 


\subsection{Near-Future Tests of the No-Hair Theorem}

In Part I of this thesis, I have developed a framework to test the no-hair theorem based a Kerr-like metric that I have designed and that contains the quadrupole moment as an independent parameter in addition to mass and spin. I have predicted observational signatures of deviations of the quadrupole moment of black holes from the Kerr value, notably in the shadows of supermassive black holes, relativistically broadened iron lines as well as quasi-periodic variability observed in both galactic black holes and active galactic nuclei. As a next step, I will expand the set of observables to include continuum fitting of accretion disk spectra, X-ray polarization, and time-resolved mapping of iron lines. My simulations will have great importance for upcoming observatories such as the Event Horizon Telescope (EHT), Astro-H, ATHENA, the Gravity and Extreme Magnetism Small Explorer (GEMS), and the Large Observatory For x-ray Timing (LOFT).

\subsubsection{Modeling Continuum Disk Spectra of Black Holes in a Kerr-like Space- time}

Fitting the continuum spectra of black-hole accretion disks is currently the most successful technique to infer black-hole spin and has measured the spins of several galactic black holes (McClintock et al. 2011). At present, all spin measurements critically rely on the assumption that astrophysical black holes are Kerr black holes. I will apply my framework to this technique and model in detail the continuum disk emission with the aim to measure the quadrupole moments of black holes. Such measurements will test the Kerr assumption in a twofold way: If we detect no deviation of the quadrupole moment from its value in the Kerr metric, it provides an independent test justifying the assumption of the Kerr met- 
ric and will increase the level of robustness of all spin measurements. If, on the other hand, quadrupole deviations turn out to be nonzero, the no-hair theorem is violated and we have a new model with which to interpret such deviations quantitatively.

Key ingredients of the continuum fitting method are the location of the ISCO, usually taken to coincide with the inner edge of the accretion disk, and the effective area of the disk projected onto the observer sky. The ISCO determines the temperature maximum and hence the measured peak flux. In Chapter 4 , I computed the location of the ISCO in the Kerr-like spacetime I designed for this purpose, and I showed that it depends strongly on both the spin and the quadrupole moment. Calculating the effective area will require a thorough analysis, which is complicated by the fact that this metric only admits three constants of the motion instead of the full set of four conserved quantities as in the Kerr metric. This can only be done numerically, and I will implement and perform these computations.

12.1.2 Development of Pattern-Recognition Techniques to Image the Shadow of Sgr A* with the Event Horizon Telescope

Submillimeter VLBI has for the first time resolved Sgr A*, the supermassive black hole in the center of the Milky Way, on scales comparable to its event horizon (Doeleman et al. 2008). Imaging of the shadow of Sgr A* is expected to be carried out within 3-5 years with a VLBI array consisting of telescopes distributed primarily in North and South America, the so-called Event Horizon Telescope (Doeleman et al. 2009a, b; Fish et al. 2009).

Shadows of black holes reveal a characteristic bright and narrow ring, which in the case of Sgr $A^{*}$ has a diameter of about $53 \mu$ arcsec. In Chapter 6, I showed that this ring depends uniquely on the mass, spin, quadrupole moment, and in- 
clination of the black hole. As the next step, I will simulate detailed images of this ring incorporating the measured intensity, which I estimated in Chapter 7.

Similar to the data analysis strategies of expected gravitational-wave signals, I will use these ring images to design pattern recognition techniques that are suited for a measurement of the parameters of Sgr $\mathrm{A}^{*}$ as well as of the supermassive black hole in M87. This is the logical next step and a refined approach compared to fitting data with accretion flow models alone, because this ring depends almost exclusively on the black hole spacetime itself and is practically independent of the underlying flow geometry.

\subsubsection{Predicting X-Ray Polarization Signatures of Black Holes in a Kerr-like Spacetime}

X-ray emission from black-hole accretion disks is expected to be polarized at a fraction of $5-10 \%$. The strong gravitational lensing near black holes imprints a characteristic signature on the observed polarization that can be used to probe the spacetime in its immediate vicinity and measure its spin (Schnittman \& Krolik 2009, 2010). The Gravity and Extreme Magnetism Small Explorer (GEMS) is a mission in NASA's Explorer program, which has been specifically designed to measure for the first time the $\mathrm{X}$-ray polarization in the emission from black holes, magnetars, pulsars, and supernova remnants. This satellite is scheduled for launch in 2014.

Using the radiative transfer code I developed in Chapter 5, I will incorporate the effect of polarization and simulate the observed signal as a function of both the spin and the quadrupole moment of black holes in order to test the no-hair theorem. I will then perform detailed simulations tailored after the GEMS design and detector response making clear predictions for the expected series of 
observations starting in 2014.

\subsubsection{Iron Line Reverberation Mapping in a Kerr-like Spacetime} Localized regions of fluorescent iron line emission follow a nearly Keplerian orbit around the black hole, and their profile appears as arcs in the time-energy plane. These signals carry the imprinted signatures of the black hole spacetime and can be used to measure its parameters (Brenneman et al. 2009). Upcoming X-ray timing missions such as the Large Observatory For x-ray Timing (LOFT) will for the first time have the sensitivity to observe iron lines in the time domain and trace these hot spots as they orbit around the black hole.

I will model the characteristic arcs in the time-energy plane in a Kerr-like spacetime as a function of the Kerr parameters and an independent quadrupole moment and predict the signatures of a potential violation of the no-hair theorem. 


\subsection{Additional Constraints on the Size of Extra Dimensions}

In Part II of this thesis, I designed a method to constrain the size of the extra dimension in RS2-type braneworld gravity. Using existing measurements of the orbital period of A0620-00 and XTE J1118+480, I obtained upper limits on the orbital period derivative and constrained the asymptotic curvature radius of the extra dimension to less than $161 \mu \mathrm{m}$.

The black hole in the binary GRO J0422+32 is another interesting and promising source. This black hole has been monitored for more than a decade (Remillard $\&$ McClintock 2006). The companion star resembles a main-sequence star with an M1 V optical counterpart (Gelino \& Harrison 2003). The orbital period is about 5.1 hours, and the black hole is relatively light with a mass of only $4 \pm 1 M_{\odot}$ (see Charles \& Coe 2006).

For this source, the effect of the predicted evaporation into the extra dimension on the orbital period becomes comparable to the effect of magnetic braking at an asymptotic curvature radius of $L \approx 20 \mu \mathrm{m}$. This is the fundamental limit that can be achieved with this source, which would improve the current table top bound (Kapner et al. 2007) by a factor of two.

Several measurements of the orbital period have been obtained to date (e.g., Webb et al. 2000), but at least one additional measurement will be required for such a tight constraint on the size of the extra dimension. 


\section{APPENDIX A}

\section{COORDINATE TRANSFORMATIONS IN THE MN SPACETIME}

I begin with the line element as given by Manko \& Novikov (1992)

$$
\begin{aligned}
d s_{\mathrm{MN}}^{2}= & -f(d t-\omega d \phi)^{2}+k^{2} f^{-1} e^{2 \gamma}\left(x^{2}-y^{2}\right) \\
& \times\left(\frac{d x^{2}}{x^{2}-1}+\frac{d y^{2}}{1-y^{2}}\right)+k^{2} f^{-1}\left(x^{2}-1\right)\left(1-y^{2}\right) d \phi^{2},
\end{aligned}
$$

where

$$
\begin{aligned}
f= & e^{2 \psi} \frac{A}{B}, \\
\omega= & 2 k e^{-2 \psi} \frac{C}{A}-\frac{4 k \alpha}{1-\alpha^{2}}, \\
e^{2 \gamma}= & e^{2 \gamma^{\prime}} \frac{A}{\left(x^{2}-1\right)\left(1-\alpha^{2}\right)^{2}}, \\
A= & \left(x^{2}-1\right)(1+u v)^{2}-\left(1-y^{2}\right)(v-u)^{2}, \\
B= & {[x+1+(x-1) u v]^{2}+[(1+y) u+(1-y) v]^{2}, } \\
C= & \left(x^{2}-1\right)(1+u v)[v-u-y(u+v)] \\
& +\left(1-y^{2}\right)(v-u)[1+u v+x(1-u v)], \\
\psi= & \beta \frac{P_{2}}{R^{3}}, \\
\gamma^{\prime}= & \frac{1}{2} \ln \frac{x^{2}-1}{x^{2}-y^{2}}+\frac{9 \beta^{2}}{6 R^{6}}\left(P_{3}^{2}-P_{2}^{2}\right) \\
& +\beta \sum_{l=0}^{2}\left[\frac{x-y+(-1)^{2-l}(x+y)}{R^{l+1}} P_{l}-2\right], \\
u= & -\alpha \exp \left[-2 \beta\left(-1+\sum_{l=0}^{2} \frac{(x-y) P_{l}}{R^{l+1}}\right)\right], \\
v= & \alpha \exp \left[2 \beta\left(1+\sum_{l=0}^{2} \frac{(-1)^{3-l}(x+y) P_{l}}{R^{l+1}}\right)\right], \\
R \equiv & \sqrt{x^{2}+y^{2}-1},
\end{aligned}
$$




$$
P_{n} \equiv P_{n}\left(\frac{x y}{R}\right), \quad P_{n}(x)=\frac{1}{2^{n} n !}\left(\frac{d}{d x}\right)^{n}\left(x^{2}-1\right)^{n} .
$$

In these equations, $k, \alpha$, and $\beta$ are free parameters, which determine the set of multipole moments of the spacetime. Note that there is a minor discrepancy in the functions $u$ and $v$ ( $a$ and $b$ in their notation) between the original form by Ref. ? and the one used by Gair et al. (2008). The difference, however, is small, and both versions satisfy the vacuum Einstein equations. I will use the version quoted by Gair et al. (2008) in this paper, but our conclusions are valid in either case. Following Gair et al. (2008), I define

$$
\begin{aligned}
\alpha & \equiv \frac{-M+\sqrt{M^{2}-a^{2}}}{a}, \\
k & \equiv M \frac{1-\alpha^{2}}{1+\alpha^{2}}=\sqrt{M^{2}-a^{2}}, \\
\beta & \equiv q \frac{M^{3}}{k^{3}},
\end{aligned}
$$

where $M$ and $a$ are the mass and the spin, respectively.

This metric can be mapped to cylindrical coordinates:

$$
\rho \equiv k \sqrt{x^{2}-1} \sqrt{1-y^{2}}, \quad z \equiv k x y
$$

with inverse

$$
\begin{aligned}
& x=\frac{1}{2 k}\left[\sqrt{\rho^{2}+(z+k)^{2}}+\sqrt{\rho^{2}+(z-k)^{2}}\right], \\
& y=\frac{1}{2 k}\left[\sqrt{\rho^{2}+(z+k)^{2}}-\sqrt{\rho^{2}+(z-k)^{2}}\right] .
\end{aligned}
$$

The MN metric then becomes

$$
d s_{\mathrm{MN}}^{2}=-f(d t-\omega d \phi)^{2}+f^{-1}\left[e^{2 \gamma}\left(d \rho^{2}+d z^{2}\right)+\rho^{2} d \phi^{2}\right] .
$$

In spherical coordinates, I define (Manko \& Novikov 1992)

$$
r \equiv k x+M, \quad \cos \theta \equiv y,
$$


and the MN metric is as given in Eq. (3.13). 


\section{APPENDIX B \\ Properties of a Kerr-Like Black Hole Spacetime}

In this appendix, I briefly summarize the explicit form and some of the properties of my metric (Chapter 4) and I derive the Keplerian and epicyclic frequencies of a particle on a circular equatorial orbit. In Boyer-Lindquist-like coordinates, the metric can be expressed by the line element

$$
\begin{aligned}
d s^{2}= & -[1+h(r, \theta)]\left(1-\frac{2 M r}{\Sigma}\right) d t^{2}-\frac{4 a M r \sin ^{2} \theta}{\Sigma}[1+h(r, \theta)] d t d \phi \\
& +\frac{\Sigma[1+h(r, \theta)]}{\Delta+a^{2} \sin ^{2} \theta h(r, \theta)} d r^{2}+\Sigma d \theta^{2} \\
& +\left[\sin ^{2} \theta\left(r^{2}+a^{2}+\frac{2 a^{2} M r \sin ^{2} \theta}{\Sigma}\right)\right. \\
& \left.+h(r, \theta) \frac{a^{2}(\Sigma+2 M r) \sin ^{4} \theta}{\Sigma}\right] d \phi^{2}
\end{aligned}
$$

where

$$
\begin{aligned}
\Sigma & \equiv r^{2}+a^{2} \cos ^{2} \theta, \\
\Delta & \equiv r^{2}-2 M r+a^{2}, \\
h(r, \theta) & \equiv \sum_{k=1}^{\infty}\left(\epsilon_{2 k}+\epsilon_{2 k+1} \frac{M r}{\Sigma}\right)\left(\frac{M^{2}}{\Sigma}\right)^{k}
\end{aligned}
$$

with the free parameters $\epsilon_{k}$. I will use this metric with only one additional parameter $\epsilon_{3}$, so that the function $h(r, \theta)$ reduces to

$$
h(r, \theta)=\epsilon_{3} \frac{M^{3} r}{\Sigma^{2}} .
$$

For a particle on a circular equatorial orbit, its energy and axial angular momentum in units of the rest mass $\mu$ of the particle are given by the expressions 
(Chapter 4)

$$
\begin{gathered}
\frac{E}{\mu}=\frac{1}{r^{6}} \sqrt{\frac{P_{1}+P_{2}}{P_{3}}} \\
\frac{L_{z}}{\mu}= \pm \frac{1}{r^{4} P_{6}}\left[\sigma \sqrt{\frac{M\left(r^{3}+\epsilon_{3} M^{3}\right) P_{5}}{P_{3}}} \mp 6 a M\left(r^{3}+\epsilon_{3} M^{3}\right) \sqrt{\frac{P_{1}+P_{2}}{P_{3}}}\right] .
\end{gathered}
$$

In these expressions, the upper signs refer to a particle that corotates with the black hole, while the lower signs refer to a counterrotating particle. The functions $P_{1}$ to $P_{6}$ are written explicitly below. Note the slight redefinition of the angular momentum $L_{z}$ and the function $P_{4}$ in the case $a<0$ with respect to Johannsen \& Psaltis (2011c). Over most of the allowed $r$-range, $\sigma=1$; close to the ISCO, however, $P_{5}$ can have an extremum at $r=r_{\sigma}$, and $\sigma=-1$ has to be chosen in the interval $r_{\mathrm{ISCO}} \leq r \leq r_{\sigma}$ for $L_{z}$ to be smooth and differentiable ${ }^{1}$.

$$
\begin{aligned}
P_{1}= & a^{2} M r^{4}\left(\epsilon_{3} M^{3}+r^{3}\right)^{2}\left\{12 \epsilon_{3} a^{2} M^{3}\left(\epsilon_{3} M^{3}+r^{3}\right)^{2}\right. \\
& -r^{4}\left[2 \epsilon_{3} M^{2} r^{3}\left(3 r^{2}-8 M^{2}\right)+\epsilon_{3}^{2} M^{5}\left(40 M^{2}-48 M r+15 r^{2}\right)\right. \\
& \left.\left.+4 r^{6}(3 r-5 M)\right]\right\} \\
P_{2}= & 2\left\{2 r^{4}\left(r^{20} \mp M P_{4}\right)+M r^{12}\left\{2 r^{9}\left(-12 M^{2}+16 M r-7 r^{2}\right)\right.\right. \\
& +\epsilon_{3} M^{2}(r-2 M)^{2} \\
& \left.\left.\times\left[\epsilon_{3}^{2} M^{6}(5 r-12 M)-6 \epsilon_{3} M^{3} r^{3}(5 M-2 r)-3 r^{6}(8 M-3 r)\right]\right\}\right\} \\
P_{3}= & r^{4}\left(12 \epsilon_{3} M^{4}-5 \epsilon_{3} M^{3} r+6 M r^{3}-2 r^{4}\right)^{2}-8 a^{2} M\left(\epsilon_{3} M^{3}+r^{3}\right)^{2} \\
& \times\left(5 \epsilon_{3} M^{3}+2 r^{3}\right) \\
P_{4}= & a\left\{M\left(\epsilon_{3} M^{3}+r^{3}\right)^{6}\left(9 \epsilon_{3}^{2} a^{2} M^{5}+16 \epsilon_{3} M^{3} r^{4}-6 \epsilon_{3} M^{2} r^{5}+4 r^{7}\right)\right. \\
& \left.\times\left[a^{2}\left(\epsilon_{3} M^{3}+r^{3}\right)+r^{4}(r-2 M)\right]^{2}\right\}^{1 / 2}
\end{aligned}
$$

\footnotetext{
${ }^{1}$ I thank H. Krawczynski for pointing this out.
} 


$$
\begin{aligned}
P_{5}= & \left(\epsilon_{3} M^{3}+r^{3}\right)\left\{12 \epsilon_{3} a^{6} M^{3}\left(\epsilon_{3} M^{3}-2 r^{3}\right)^{2}\left(\epsilon_{3} M^{3}+r^{3}\right)^{4}\right. \\
& +a^{4} r^{4}\left(\epsilon_{3} M^{3}+r^{3}\right)^{2}\left(-40 \epsilon_{3}^{4} M^{13}+40 \epsilon_{3}^{4} M^{12} r-15 \epsilon_{3}^{4} M^{11} r^{2}\right. \\
& +128 \epsilon_{3}^{3} M^{10} r^{3}-296 \epsilon_{3}^{3} M^{9} r^{4}+54 \epsilon_{3}^{3} M^{8} r^{5}-924 \epsilon_{3}^{2} M^{7} r^{6}+276 \epsilon_{3}^{2} M^{6} r^{7} \\
& -36 \epsilon_{3}^{2} M^{5} r^{8}-880 \epsilon_{3} M^{4} r^{9}+304 \epsilon_{3} M^{3} r^{10}-24 \epsilon_{3} M^{2} r^{11}-112 M r^{12} \\
& \left.+16 r^{13}\right)-2 a^{2} r^{8}\left[48 \epsilon_{3}^{5} M^{17}-12 \epsilon_{3}^{5} M^{16} r-52 \epsilon_{3}^{5} M^{15} r^{2}\right. \\
& +3 \epsilon_{3}^{4} M^{14} r^{3}\left(5 \epsilon_{3}+88\right)-720 \epsilon_{3}^{4} M^{13} r^{4}+298 \epsilon_{3}^{4} M^{12} r^{5} \\
& -3 \epsilon_{3}^{3} M^{11} r^{6}\left(13 \epsilon_{3}+480\right)+516 \epsilon_{3}^{3} M^{10} r^{7}+2 \epsilon_{3}^{3} M^{9} r^{8} \\
& -6 \epsilon_{3}^{2} M^{8} r^{9}\left(3 \epsilon_{3}+508\right)+2292 \epsilon_{3}^{2} M^{7} r^{10}-628 \epsilon_{3}^{2} M^{6} r^{11} \\
& +12 \epsilon_{3} M^{5} r^{12}\left(5 \epsilon_{3}-134\right)+1188 \epsilon_{3} M^{4} r^{13}-296 \epsilon_{3} M^{3} r^{14} \\
& \left.+24 M^{2} r^{15}\left(\epsilon_{3}-9\right)+120 M r^{16}-16 r^{17}\right] \\
& -r^{14}\left(\epsilon_{3} M^{3}+6 M r^{2}-2 r^{3}\right)^{2}\left(96 \epsilon_{3}^{2} M^{7}-76 \epsilon_{3}^{2} M^{6} r+15 \epsilon_{3}^{2} M^{5} r^{2}\right. \\
& \left.\left.+72 \epsilon_{3} M^{4} r^{3}-44 \epsilon_{3} M^{3} r^{4}+6 \epsilon_{3} M^{2} r^{5}+12 M r^{6}-4 r^{7}\right)\right\} \\
& \mp 4 P_{4}\left[a^{2}\left(\epsilon_{3} M^{3}-2 r^{3}\right)^{2}\left(\epsilon_{3} M^{3}+r^{3}\right)\right. \\
& \left.+6 \epsilon_{3} M^{3} r^{5}\left(\epsilon_{3} M^{3}+6 M r^{2}-2 r^{3}\right)\right] \\
P_{6}= & -\epsilon_{3}^{2} M^{6}-6 \epsilon_{3} M^{4} r^{2}+\epsilon_{3} M^{3} r^{3}-6 M r^{5}+2 r^{6}
\end{aligned}
$$

Next, I derive the Keplerian orbital frequency of a particle with energy $E$ and axial angular momentum $L_{z}$. A particle with a rest mass $\mu$ has the 4-momentum

$$
p^{\alpha}=\mu \frac{d x^{\alpha}}{d \tau},
$$

where $E=-p_{t}$ and $L_{z}=p_{\phi}$. Hereafter, I will set the rest mass equal to unity. Using the conservation of the energy and axial angular momentum, I solve for the momentum components $p^{t}$ and $p^{\phi}$ and obtain

$$
p^{t}=-\frac{g_{\phi \phi} E+g_{t \phi} L_{z}}{g_{t t} g_{\phi \phi}-g_{t \phi}^{2}},
$$




$$
p^{\phi}=\frac{g_{t \phi} E+g_{t t} L_{z}}{g_{t t} g_{\phi \phi}-g_{t \phi}^{2}} .
$$

For the Keplerian frequency, I derive the expression

$$
\Omega_{\phi} \equiv \frac{d \phi}{d t}=\frac{p^{\phi}}{p^{t}} \equiv \pm \frac{Q_{1}}{Q_{2}},
$$

where

$$
\begin{aligned}
Q_{1} \equiv & r\left(\Sigma^{2}+\epsilon_{3} r M^{3}\right)[ \pm 2 a M P 6 \sqrt{P 1+P 2}+r(2 M r-\Sigma) \\
& \left.\left( \pm 6 a M\left(r^{3}+\epsilon_{3} M^{3}\right) \sqrt{P 1+P 2}-\sqrt{M\left(r^{3}+\epsilon_{3} M^{3}\right) P 5}\right)\right] \\
Q_{2} \equiv 2 a M r^{3}\left(\Sigma^{2}+\epsilon_{3} r M^{3}\right) & \\
& {\left[6 a M\left(r^{3}+\epsilon_{3} M^{3}\right) \sqrt{P 1+P 2} \mp \sqrt{M\left(r^{3}+\epsilon_{3} M^{3}\right) P 5}\right] } \\
+ & P 6 \sqrt{P 1+P 2}\left[\left(r^{2}+a^{2}\right) \Sigma^{3}+a^{2} M r\left(2 \Sigma^{2}+\epsilon_{3} r M^{2}(r+2 M)\right)\right]
\end{aligned}
$$

In the case $\epsilon_{3}=0$, this expression reduces to the familiar Kerr Keplerian frequency

$$
\Omega_{\phi}^{K}= \pm \frac{\sqrt{M}}{r^{3 / 2} \pm a \sqrt{M}} .
$$

Finally, I compute the radial and vertical epicyclic frequencies as well as the Lense-Thirring frequency. My derivation is analogous to the one in Johannsen \& Psaltis (2011a). I use the conservation of the particle's rest mass to define an effective potential

$$
V_{\mathrm{eff}} \equiv \frac{1}{2}\left[-g_{\mathrm{tt}}\left(p^{\mathrm{t}}\right)^{2}-2 g_{\mathrm{t} \phi} p^{\mathrm{t}} p^{\phi}-g_{\phi \phi}\left(p^{\phi}\right)^{2}-1\right] .
$$

Radial and vertical motions around a circular equatorial orbit at a radius $r_{0}$ are governed by the equations

$$
\begin{aligned}
& \frac{1}{2}\left(\frac{d r}{d t}\right)^{2}=\frac{V_{\mathrm{eff}}}{g_{\mathrm{rr}}\left(p^{t}\right)^{2}} \equiv V_{\mathrm{eff}}^{\mathrm{r}}, \\
& \frac{1}{2}\left(\frac{d \theta}{d t}\right)^{2}=\frac{V_{\mathrm{eff}}}{g_{\theta \theta}\left(p^{\mathrm{t}}\right)^{2}} \equiv V_{\mathrm{eff}}^{\theta},
\end{aligned}
$$


respectively.

I, then, introduce small perturbations $\delta r$ and $\delta \theta$ and take the coordinate time derivative of Equations (B.22) and (B.23), which yields

$$
\begin{aligned}
\frac{d^{2}(\delta r)}{d t^{2}} & =\frac{d^{2} V_{\text {eff }}^{\mathrm{r}}}{d r^{2}} \delta r \\
\frac{d^{2}(\delta \theta)}{d t^{2}} & =\frac{d^{2} V_{\mathrm{eff}}^{\theta}}{d \theta^{2}} \delta \theta .
\end{aligned}
$$

From these expressions, I derive the radial and vertical epicyclic frequencies as

$$
\begin{aligned}
& \kappa_{\mathrm{r}}^{2}=-\frac{d^{2} V_{\mathrm{eff}}^{\mathrm{r}}}{d r^{2}}, \\
& \Omega_{\theta}^{2}=-\frac{d^{2} V_{\mathrm{eff}}^{\theta}}{d \theta^{2}},
\end{aligned}
$$

where the second derivatives are evaluated at $r=r_{0}$. These expressions are lengthy, and I do not write them here explicitly. Having the expressions of the Keplerian and vertical epicyclic frequencies at hand, the Lense-Thirring frequency is given by

$$
\Omega_{\mathrm{LT}} \equiv \Omega_{\phi}-\Omega_{\theta} .
$$




\section{APPENDIX C \\ PHOTON INITIAL CONDITIONS}

For the geometry in Figure 6.1, the initial conditions of the photons in the image plane of a distant observer are better expressed in spherical coordinates. The image plane is located at a distance $d$ from the black hole at an inclination angle $i$. Since the observer is far away from the black hole, I can express the initial conditions using standard Euclidean geometry.

First, I transform the image plane coordinates $\left(x^{\prime}, y^{\prime}, z^{\prime}\right)$ into Cartesian coordinates $(x, y, z)$ centered at the black hole. I find

$$
\begin{aligned}
& x=-y^{\prime} \cos i+z^{\prime} \sin i+d \sin i, \\
& y=x^{\prime}, \\
& z=y^{\prime} \sin i+z^{\prime} \cos i+d \cos i .
\end{aligned}
$$

I then convert the Cartesian black-hole coordinates into spherical coordinates by the usual transformation

$$
\begin{gathered}
r=\sqrt{x^{2}+y^{2}+z^{2}}, \\
\theta=\arccos \frac{z}{r}, \\
\phi=\arctan \frac{y}{x} .
\end{gathered}
$$

The initial conditions for a photon on the image plane at $\left(x^{\prime}, y^{\prime}\right)$ with uniform initial momentum $\vec{k}_{0}=-k_{0} \hat{z}^{\prime}$ are then given by

$$
\begin{gathered}
r_{0}=\sqrt{x^{\prime 2}+y^{\prime 2}+d^{2}}, \\
\theta_{0}=\arccos \frac{y^{\prime} \sin i+d \cos i}{r_{0}},
\end{gathered}
$$




$$
\phi_{0}=\arctan \frac{x^{\prime}}{d \sin i-y^{\prime} \cos i},
$$

and

$$
\begin{gathered}
k_{r_{0}}=-\frac{d}{r_{0}} k_{0}, \\
k_{\theta_{0}}=\frac{\cos i-\left(y^{\prime} \sin i+d \cos i\right) \frac{d}{r_{0}^{2}}}{\sqrt{x^{\prime 2}+\left(d \sin i-y^{\prime} \cos i\right)^{2}}} k_{0}, \\
k_{\phi_{0}}=\frac{x^{\prime} \sin i}{x^{\prime 2}+\left(d \sin i-y^{\prime} \cos i\right)^{2}} k_{0},
\end{gathered}
$$

where $r_{0}, \theta_{0}$, and $\phi_{0}$ are the initial coordinates of a given photon, and $k_{\mathrm{r}_{0}}, k_{\theta_{0}}$, and $k_{\phi_{0}}$ are the components of its initial 3-momentum. The time component of the 4 -momentum is calculated from the 3-momentum $\vec{k}_{0}$ so that its norm vanishes. 


\section{REFERENCES}

Abramowicz, M. A., \& Kluźniak, W. 2001, A\&A, 374, L19

Abramowicz, M. A., Karas, V., Kluźniak, W., Lee, W. H., \& Rebusco, P. 2003, PASJ, 55,467

Adelberger, E. G., Heckel, B. R., Hoedl, S., Hoyle, C. D., Kapner, D. J., \& Upadhye, A. 2007, PRL 98,131104

Agol, E. \& Krolik, J. H. 2000, ApJ 528, 161

Alexander, S., \& Yunes, N. 2009, Phys. Rept., 480, 1

Antoniadis, I., Arkani-Hamed, N., Dimopoulos, S., \& Dvali, G. 1998, Phys. Lett. B, 436, 257

Apostolatos, T. A., Lukes-Gerakopoulos, G. \&, Contopoulos, G. 2009, Phys. Rev. Lett., 103, 111101

Arkani-Hamed, N., Dimopoulos, S., \& Dvali, G. 1998, Phys. Lett. B 429, 263

Aschenbach, B., Grosso, N., Porquet, D., \& Predehl, P. 2004, A\&A, 417, 71

Bäckdahl, T. \& Herberthson, M. 2006, Class. Quantum Grav., 23, 5997

Baganoff, F. K., et al. 2001, Nature, 413, 45

Bambi, C. \& Freese, K. 2009, Phys. Rev. D, 79, 043002

Bambi, C. 2011, Phys. Rev. D,83, 103003 
Bambi, C., \& Modesto, L. 2011, Phys. Lett. B, 706, 13

Bambi, C., \& Barausse, E. 2011, ApJ, 731, 121

Bambi, C. 2012a, Phys. Rev. D, 85, 043001

Bambi, C. 2012b, Phys. Rev. D, 85, 043002

Bao, G., Hadrava, P., \& Østgaard, E. 1994, ApJ, 435, 55

Barack, L., \& Cutler, C. 2004, Phys. Rev. D, 69, 082005

Barack, L., \& Cutler, C. 2007, Phys. Rev. D, 75, 042003

Barceló, C., Liberati, S., Sonego, S., \& Visser, M. 2008, Phys. Rev. D, 77, 044032

Bardeen, J. M. in Black Holes, eds. DeWitt, DeWitt, Gordon and Breach, 1973

Bardeen, J. M., Press, W. H., \& Teukolsky, S. A. 1972, ApJ, 178, 347

Beckwith, K. \& Done, C. 2004, MNRAS 352, 353

Beckwith, K. \& Done, C. 2005, MNRAS 359, 1217

Beig, R. \& Simon, W. 1980, Commun. Math. Phys., 78, 75

Beig, R. \& Simon, W. 1981, Proc. Roy. Soc. London A, 376, 333

Bélanger, G., Terrier, R., de Jager, O. C., Goldwurm, A., \& Melia, F. 2006, J. Phys. Conf. Ser., 54, 420

Bendo, G. J., et al. 2006, ApJ, 645, 134

Benlloch, S., Wilms, J., Edelson, R., Yaqoob, T., \& Staubert, R. 2001, ApJ, 562, L121 
Bennett, C. L., et al. 2003, ApJS, 148, 97

Berti, E., White, F., Maniopoulou, A., \& Bruni, M. 2005, MNRAS, 358, 923

Berti, E., \& Stergioulas, N. 2004, MNRAS, 350, 1416

Bertotti, B., Iess, L., \& Tortora, P. 2003, Nature, 425, 374

Bini, D., Geralico, A., Luongo, O., \& Quevedo, H. 2009, Class. Quantum Grav., 26,5006

Birkhoff, G. D. 1913, Trans. Am. Math. Soc., 14, 14

Bower, G. C., Goss, W. M., Falcke, H., Backer, D. C., \& Lithwick, Y. 2006, ApJ, 648, L127

Bozza, V. 2008, Phys. Rev. D 78, 063014

Bozza, V. 2009, arXiv:0911.2187

Bradley, M. \& Fodor, G. 2009, Phys. Rev. D 79, 044018

Braje, T. M., Romani, R. W., \& Rauch, K. P. 2000, ApJ, 531, 447

Brans, C., \& Dicke, R. H. 1961, Phys. Rev., 124, 925

Brenneman, L. W. et al., Astro 2010 Science White Paper, arXiv:0902.4691

Brenneman, L. W., \& Reynolds, C. S. 2009, ApJ, 702, 1367

Brenneman, L. W., \& Reynolds, C. S. 2006, ApJ, 652, 1028

Brink, J. 2008, Phys. Rev. D, 78, 102001 
Brink, J. 2009, arXiv:0911.4161

Broderick, A. E. \& Loeb, A. 2005, MNRAS, 363, 353

Broderick, A. E. \& Loeb, A. 2006, MNRAS, 367, 905

Broderick, A. E. \& Loeb, A. 2009, ApJ 697, 1164

Broderick, A. E., Fish, V. L., Doeleman, S. S., \& Loeb, A. 2009a, ApJ, 697, 45

Broderick, A. E., Loeb, A. \&, Narayan, R. 2009b, ApJ, 701, 1357

Broderick, A. E., Fish, V. L., Doeleman, S. S., \& Loeb, A. 2011, ApJ, 735, 110

Broderick, A. E., Johannsen, T., Loeb, A., \& Psaltis, D., 2012, in preparation

Burgay, M., et al. 2003, Nature, 426, 531

Cadeau, C., Morsink, S. M., Leahy, D., \& Campbell, S. S. 2007, ApJ, 654, 458

Čadež, A., Fanton, C., \& Calvani, M. 1998, New Astronomy, 3, 647

Chandrasekhar, S., The Mathematical Theory of Black Holes, Oxford University Press, 1983

Charles, P. A., Coe, M. J., in "Compact Stellar X-ray Sources", eds. W.H.G. Lewin and M. van der Klis (Cambridge University Press), arXiv:0308020

Chen, S., \& Jing, J., arXiv:1110.3462

Carroll, S. M., Spacetime and geometry: An introduction to general relativity (Addison-Wesley, San Francisco, 2004), p. 513

Carter, B. 1968, Phys. Rev., 174, 5 
Carter, B. 1971, Phys. Rev. Lett. 26, 331

Carter, B. in Black Holes, ed. DeWitt, DeWitt, Gordon and Breach, 1973

Chan, C.-K. 2009, ApJ, 704, 68

Clarke, C. J. S., \& de Felice, F. 1982, J. Phys. A, 15, 2415

Collins, N. A. \& Hughes, S. A. 2004, Phys. Rev. D 69, 124022

Cunningham, C. 1976, ApJ, 208, 534

Dai, D.-C., \& Stojkovic, D., arXiv:1004.3291

Damour, T., \& Esposito-Farèse, G. 1996, Phys. Rev. D, 54, 1474

Damour, T., \& Esposito-Farèse, G. 1993, Phys. Rev. Lett., 70, 2220

Damour, T., \& Taylor, J. H. 1992, Phys. Rev. D, 45, 1840

Dauser, T., Wilms, J., Reynolds, C. S., \& Brenneman, L. W. 2010, MNRAS, 409, 1534

DeDeo, S., \& Psaltis , D. 2003, Phys. Rev. Lett., 90, 141101

de Kool, M., van den Heuvel, E. P. J., Pylyser, E. 1987, 1987, A\&A, 183

Dexter, J. \& Agol, E. 2009, ApJ 696, 1616

Dexter, J., Agol, E., \& Fragile, P. C. 2009, ApJ, 703, L142

Dexter, J., McKinney, J. C., \& Agol, E. 2011, arXiv:1109.6011

Dietz, W. in Solutions of Einstein's Equations, Techniques and Results, eds. Hoenselaers, Dietz, Springer, 1984 
Di Matteo, T., Fabian, A. C., Carilli, L. C., \& Ivison, J. R. 1999, Adv. Space Res., 23, 1075

Di Matteo, T., Quataert, E., Allen, S. W., Narayan, R., \& Fabian, A. C. 2000, MNRAS, 311, 507

Doeleman, S. S., Fish, V. L., Broderick, A. E., Loeb, A., \& Rogers, A. E. E. 2009a, ApJ, 695, 59

Doeleman, S. S., et al. 2009b, Astro2010: The Astronomy and Astrophysics Decadal Survey, Science White Papers, no. 68

Doeleman, S. S. et al. 2008, Nature, 455, 78

Doi, A., Kameno, S., Kohno, K., Nakanishi, K., \& Inoue, M. 2005, MNRAS, 363, 692

Dolence, J. C., Gammie, C. F., Mościbrodzka, M., \& Leung, P. K. 2009, ApJS, 184, 387

Dovčiak, M., Karas, V., \& Yaqoob, T. 2004, ApJS, 153, 205

Drake, S. P., \& Szekeres, P. 2000, Gen. Rel. Grav. 32, 445

Eggleton, P. P. 1983, ApJ, 268, 368

Eisenhauer, F., et al. 2011, Msngr., 143, 16

Emparan, R., Fabbri, A., \& Kaloper, N. 2002, JHEP 0208, 043

Emparan, R., García-Bellido, J., \& Kaloper, N. 2003, JHEP 0301, 079

Ernst, F. J. 1968, Phys. Rev., 167, 1175 
Everitt, C. W. F., et al. 2011, Phys. Rev. Lett., 106, 221101

Fabian, A. C., et al. 2009, Nature, 459, 540

Fabian, A. C., Rees, M. J., Stella, L., \& White, N. E. 1989, MNRAS, 238, 729

Falcke, H., Melia, F., \& Agol, E. 2000, ApJ 528, L13

Falcke, H., Mannheim, K., \& Biermann, P. L. 1993, A\&A, 278, L1

Fanton, C., Calvani, M., de Felice, F., \& Čadež, A. 1997, PASJ 49, 159

Fish, V. L., et al. 2011, ApJ, 727, 36

Fish, V. L., \& Doeleman, S. S. 2009, in IAU Symp. 261, Relativity in Fundamental Astronomy: Dynamics, Reference Frames, and Data Analysis, ed. S. Klioner, P. K. Seidelmann, \& M. Soffel (Cambridge: Cambridge Univ. Press)

Fitzpatrick, A. L., Randall, L., \& Wiseman, T. 2006, JHEP 0611, 033

Fodor, G., Hoenselaers, C., \& Perjés, Z. 1989, J. Math. Phys., 30, 2252

Frank, J., King, A. R., \& Raine D. 2002, Accretion Power in Astrophysics (Cambridge University Press)

Friedberg, R., Lee, T. D., \& Pang, Y. 1987, Phys. Rev. D35, 3640

Friedrich, H., Rácz, I., \& Wald, R. M. 1999, Commun. Math. Phys., 204, 691

Froeschlé, M., Mignard, F., \& Arenou, F. 1997, in Proceedings from the Hipparcos Venice '97 Symposium (Noordwijk: ESA)

Gair, J. R., \& Yunes, N. 2011, Phys. Rev. D, 84, 064016 
Gair, J. R., Li, C., \& Mandel, I. 2008, Phys. Rev. D 77, 024035

Gelino, D. M., \& Harrison, T. E., 2003, ApJ, 599, 1254

Genzel, R., Schödel, R., Ott, T., Eckart, A., Alexander, T., Lacombe, F., Rouan, D., \& Aschenbach, B. 2003, Nature, 425, 934

Geraci, A. A., Smullin, S. J., Weld, D. M., Chiaverini, J., \& Kapitulnik, A. 2008, Phys. Rev. D in press, arXiv:0802.2350.v1

Geroch, R. 1970, J. Math. Phys. 11, 2580

Ghez, A. M. et al. 2008, ApJ, 689, 1044

Ghez, A. M., et al. 2004, ApJ, 601, L159

Gierliński, M., Middleton, M., Ward, M., \& Done, C. 2008, Nature, 455, 369

Gillessen, S., Eisenhauer, F., Trippe, S., Alexander, T., Genzel, R., Martins, F., \& Ott, T. 2009, ApJ, 692, 1075

Glampedakis, K. \& Babak, S. 2006, Class. Quantum Grav. 23, 4167

Gödel, K. 1949, Rev. Mod. Phys. 21, 447

González Hernández, J. I., Rebolo, R., Israelian, G., \& Casares, J. 2004, ApJ, 609, 988

Gott, J. R. 1991, Phys. Rev. Lett., 66, 1126

Gültekin, K., Cackett, E. M., Miller, J. M., Di Matteo, T., Markoff, S., \& Richstone, D. O. 2009, ApJ, 706, 404 
Hansen, R. O. 1974, J. Math. Phys. 15, 46

Hartle, J. B. 2003, Gravity, An Introduction to Einstein's General Relativity (San Francisco: Addison Wesley)

Hartle, J. B., \& Thorne, K. S. 1968, ApJ, 153, 807

Hartle, J. B. 1967, ApJ, 150, 1005

Hauser, I. \& Ernst, F. J. 1981, J. Math. Phys., 22, 1051

Hawking, S. W. 1972, Commun. Math. Phys. 25, 152

Heusler, M., Black Hole Uniqueness Theorems (Cambridge University Press, Cambridge, 1996)

Hirabayashi, H., et al. 1998, Science, 281, 1825

Ho, L. C. 2009, ApJ, 699, 626

Hodge, P. W. 1981, A\&AA, 19, 357

Hornstein, S. D., Matthews, K., Ghez, A. M., Lu, J. R., Morris, M., Becklin, E. E., Rafelski, M., \& Baganoff, F. K. 2007, ApJ, 667, 900

Hughes, S. A. 2010, arXiv:1002.2591

Hughes, S. A. 2006, AIP Conf. Proc. 873, 233

Hulse, R. A., \& Taylor, J. H. 1975, ApJ, 195, L51

Israel, W. 1967, Phys. Rev. 164, 1776

Israel, W. 1968, Commun. Math. Phys. 8, 245 
Israel, F. P., Raban, D., Booth, R. S., \& Rantakyrö, F. T. 2008, A\&A, 483, 741

Jacobi, G. H., et al. 1992, PASP, 104, 599

Johannsen , T. 2012, Adv. Astron., 2012, 1

Johannsen, T., Psaltis, D., Gillessen, S., Marrone, D. P., Özel, F, Doeleman, S. S., \& Fish, V. L. 2012a, submitted to ApJ, arXiv:1201.0758

Johannsen, T., Vigeland, S. J., Yunes, N., Hughes, S. A., \& Psaltis, D. 2012b, in preparation

Johannsen, T., \& Psaltis, D. 2011a, Adv. Space Res., 47, 528

Johannsen, T., \& Psaltis, D. 2011b, ApJ, 726, 11

Johannsen, T., \& Psaltis, D. 2011c, Phys. Rev. D, 83, 124015

Johannsen, T., \& Psaltis, D. 2010a, ApJ, 716, 187

Johannsen, T., \& Psaltis, D. 2010b, ApJ, 718, 446

Johannsen, T. 2009, A\&A, 507, 617

Johannsen, T., Psaltis, D., \& McClintock, J. E., 2009, ApJ, 691, 997

Justham, S., Rappaport, S., \& Podsiadlowski, P. 2006, MNRAS, 366, 1415

Kalogera, V., \& Webbink, R. F. 1996, ApJ, 458, 301

Kapner, D. J., Cook, T. S., Adelberger, E. G., Gundlach, J. H., Heckel, B. R., Hoyle, C. D., \& Swanson, H. E. 2007, Phys. Rev. Lett. 98, 021101

Karas, V., \& Sochora, V. 2010, ApJ, 725, 1507 
Kato, S. 1990, Publ. Astron. Soc. Japan, 42, 99

Kato, S., \& Fukue, J. 1980, PASJ, 32, 377

Kardashev, N. S. 2009, Phys. U., 52, 1127

Kelley, R. L., Rappaport, S., Clark, G. W., \& Petro, L. D. 1982, ApJ, 268, 790

Kennefick, D., \& Ó Murchadha, N. 1995, Class. Quantum Grav. 12, 149

Kerr, R. P. 1963, Phys. Rev. Lett., 11, 237

Kluźniak, W., \& Abramowicz, M. A., arXiv:0105057

Kormendy, J. \& Richstone, D. 1995, ARAA, 33, 581

Kramer, M., et al. 2006, Science, 314, 97

Laarakkers, W. G., \& Poisson, E. 1999, ApJ, 512, 282

Lamoreaux, S. K., Jacobs, J. P., Heckel, B. R., Raab, F. J., \& Fortson, E. N. 1986, Phys. Rev. Lett., 57, 3125

Lanczos, K. 1924, Zeitschr. f. Phys. A, 21, 73

Laor, A. 1991, ApJ, 376, 90

Laor, A., Netzer, H., \& Piran, T. 1990, MNRAS, 242, 560

Lebach, D. E., Corey, B. E., Shapiro, I. I., Ratner, M. I., Webber, J. C., Rogers, A. E. E., Davis, J. L., \& Hering, T. A. 1995, Phys. Rev. Lett., 75, 1439

Lee, S.-S., et al. 2008, ApJ, 136, 159 
Leeuw, L. L., Sansom, A. E., Robson, E. I., Haas, M., \& Kuno, N. 2004, ApJ, 612, 837

Li, L.-X., Goodman, J., \& Narayan, R. 2003, ApJ, 593, 980

Li, C., \& Lovelace, G. 2008, Phys. Rev. D, 77, 064022

Liu, K., Wex, N., Kramer, M., Cordes, J. M., \& Lazio, T. J. W. 2012, ApJ, 747, 1

Lorimer, D. R. 2005, Liv. Rev. Rel., 8, 7

Lukes-Gerakopoulos, G., Apostolatos, T. A., \& Contopoulos, G. 2010, Phys. Rev. D, 81, 124005

Lyne, A. G., et al. 2004, Science, 303, 1153

Mahadevan, R. 1998, Nature, 394, 651

Manko, V. S. \& Novikov, I. D. 1992, Class. Quantum Grav., 9, 2477

Manko, V. S. \& Sibgatullin, N. R. 1993, Class. Quantum Grav., 10, 1383

Mao, S. A., Psaltis, D., \& Milsom, J. A. 2009, ApJ, 703, 717

Marrone, D. P., Moran, J. M., Zhao, J.-H., \& Rao, R. 2006, J. Phys. Conf. Ser., 54, 354

Marsh, T. R., Robinson, E. L., \& Wood, J. H. 1994, MNRAS, 266, 137

Maartens, R., 2004, LRR, 7, 7

Mazur, P. O. 1982, J. Phys. A, 15, 3173

Mazur, P. O. \& Mottola, E. 2001, arXiv:0109035 
McClintock, J. E., et al. 2011, Class. Quantum Grav., 28, 4009

McClintock, J. E., \& Remillard, R. A. 1986, ApJ, 308, 110

McClintock, J. E., Garcia, M. R., Caldwell, N., Falco, E. E., Garnavich, P. M., \& Zhao, P., 2001, ApJ, 551, 147

McClintock, J. E. \& Remillard, R. A. in Compact Stellar X-Ray Sources, eds. Lewin, van der Klis, Cambridge University Press, 2006

McClintock, J. E., Narayan, R., Gou, L., Penna, F., \& Steiner, J. A. 2009, submitted to the proceedings of the conference "X-ray Astronomy 2009: Present Status, Multi-Wavelength Approach and Future Perspectives," AIP, eds. A. Comastri et al., arXiv:0911.5408

Mecheri, R., Abdelatif, T., Irbah, A., Provost, J., \& Berthomieu, G. 2004, Solar Phys., 222, 191

Merritt, D., Alexander, T., Mikkola, S., \& Will, C. M. 2010, Phys. Rev. D, 81, 062002

Meyer, L., Schödel, R., Eckart, A., Karas, V., Dovčiak, M., \& Duschl, W. J. 2006, A\&A, 458, L25

Middelberg, E., Roy, A. L., Walker, R. C., \& Falcke, H. 2005, A\&A, 433, 897

Miller, J. 2007, ARA\&A, 45, 441

Miller, M. C., \& Lamb, F. K. 1998, ApJL, 499, L37

Morsink, S. M., Leahy, D. A., Cadeau, C., \& Braga, J. 2007, ApJ, 663, 1244 
Mościbrodzka, M., Gammie, C. F., Dolence, J. C., Shiokawa, H., \& Leung, P. K. 2009, ApJ, 706, 497

Muno, M. P., Özel, F., \& Chakrabarty, D. 2002, ApJ, 581, 550

Murdin, P., Allen, D. A., Morton, D. C., Whelan, J. A. J., \& Thomas, R. M. 1980, MNRAS 192, 709

Narayan, R. \& Heyl, J. S. 2002, ApJ, 574, L139

Narayan, R., Garcia, M. R., \& McClintock, J. E. 2001, in Proc. IX Marcel Grossmann Meeting, ed. V. Gurzadyan, R. Jantzen, \& R. Ruffini (Singapore: World Scientific), arXiv:0107387

Narayan, R., Garcia, M. R., \& McClintock, J. E. 1997, ApJ, 478, L79

Narayan, R., \& Yi, I. 1995, ApJ, 444, 231

Narayan, R., Yi, I., \& Mahadevan, R. 1995, Nature, 374, 623

Narayan, R., \& Yi, I. 1994, ApJL, 428, L13

Neilsen, J., Steeghs, D., \& Vrtilek, S. D. 2008, MNRAS, 384, 849

Newman, E. T., Couch, K., Chinnapared K., Exton, A., Prakash, A., \& Torrence, R. 1965, J. Math. Phys., 6, 918

Newman, E. T., \& Janis, A. I. 1965, J. Math. Phys. 6, 915

Newman, E. T., \& Penrose, R. 1962, J. Math. Phys. 3, 566

Noble, S. C., Krolik, J. H., \& Hawley, J. F. 2010, ApJ, 711, 959 
Nordtvedt, K. 1968, Phys. Rev., 169, 1014

Nowak, M. A., \& Wagoner, R. V. 1991, ApJ, 378, 656

Nowak, M. A., \& Wagoner, R. V. 1992, ApJ, 393, 697

Nowak, M. A., \& Wagoner, R. V. 1993, ApJ, 418, 187

Okazaki, A. T., Kato, S., \& Fukue, J. 1987, Publ. Astron. Soc. Japan, 39, 457

Oke, J. B. 1977, ApJ, 217, 181

Oppenheimer, J. R. \& Snyder, H. 1939, Phys. Rev., 56, 455

Orosz, J. A., Bailyn, C. D., Remillard, R. A., McClintock, J. E., \& Foltz, C. B. 1994, ApJ, 436, 848

Ortega-Rodríguez, M., Silbergleit, A. S., \& Wagoner, R. V. 2002, ApJ, 567, 1043

Özel, F., \& Psaltis, D. 2003, ApJL, 582, L31

Özel, F., Psaltis, D., \& Narayan, R. 2000, ApJ, 541, 234

Pani, P., \& Cardoso, V. 2009, Phys. Rev. D, 79, 084031

Papapetrou, A. 1953, Ann. Physik, 447, 309

Pechenick, K. R., Ftaclas, C., \& Cohen, J. M. 1983, ApJ, 274, 846

Pellegrini, S., Venturi, T., Comastri, A., Fabbiano, G., Fiore, F., Vignali, C., Morganti, R., \& Trinchieri, G. 2003, ApJ, 585, 677

Penrose, R. 1969, Riv. del Nouvo Cimento, 1, 252 
Perez, C. A., Silbergleit, A. S., Wagoner, R. V., \& Lehr, D. E. 1997, ApJ, 476, 589

Poisson, E., A Relativist's Toolkit (Cambridge University Press, Cambridge, 2004)

Postnov, K. A., \& Cherepashchuk, A. M. 2003, Astron. Rep. 80, 1075

Poutanen, J., \& Beloborodov, A. M. 2006, MNRAS, 373, 836

Price, R. H. 1972a, Phys. Rev. D, 5, 2419

Price, R. H. 1972b, Phys. Rev. D, 5, 2439

Psaltis, D. 2003 in X-ray Timing 2003: Rossi and Beyond, ed. P. Kaaret, F. K. Lamb, \& J. H. Swank (Melville: AIP Conf. Proc.)

Psaltis, D. in Compact Stellar X-Ray Sources, eds. Lewin, van der Klis, Cambridge University Press, 2006

Psaltis, D. 2007, Phys. Rev. Lett., 98, 181101

Psaltis, D. 2008a, Liv. Rev. Rel., 11, 9

Psaltis, D. 2008b, ApJ, 688, 1282

Psaltis, D., Perrodin, D., Dienes, K. R., \& Mocioiu, I. 2008, Phys. Rev. Lett. 100, 091101

Psaltis, D. 2009, J. Phys. Conf. Ser. 189, 012033

Psaltis, D., \& Johannsen, T. 2011, J. Phys. Conf. Ser., 283, 012030

Psaltis, D., \& Johannsen, T. 2012, ApJ, 745, 1

Randall, L., \& Sundrum, R. 1999, Phys. Rev. Lett. 83, 4690 
Rappaport, S., Verbunt, F., \& Joss, P. C. 1983, ApJ, 275, 713

Rauch, K. P., \& Blandford, R. D. 1994, ApJ, 421, 46

Reasenberg, R. D., et al. 1979, ApJ, 234, L219

Remillard, R. A., \& McClintock, J. E. 2006, ARAA, 44, 49

Reynolds, C. S., \& Miller, M. C. 2009, ApJ, 692, 869

Reynolds, C. S., \& Fabian, A. C. 2008, ApJ, 675, 1048

Reynolds, C. S., \& Nowak, M. A. 2003, Phys. Reports, 377, 389

Rezzolla, L., Yoshida, S'i., Maccarone, T. J., \& Zanotti, O. 2003, MNRAS, 344, L37

Robinson, D. C. 1975, Phys. Rev. Lett. 34, 905

Rodriguez, C. L., Mandel, I., \& Gair, J. R., arXiv:1112.1404

Ryan, F. D. 1995, Phys. Rev. D 52, 5707

Ryan, F. D. 1997a, Phys. Rev. D 56, 1845

Ryan, F. D. 1997b, Phys. Rev. D 56, 7732

Sadeghian, L., \& Will, C. M. 2011, Class. Quantum Grav., 28, 5029

Sadler, E. M., et al. 2008, MNRAS, 385, 1656

Sanabria-Goḿez, J. D., Hernández-Pastora, J. L., \& Dubeibe, F. L. 2010, arXiv:1009.0320

Schlamminger, S., Choi, K.-Y., Wagner, T. A., Gundlach, J. H., \& Adelberger, E. G. 2008, Phys. Rev. Lett., 100, 041101 
Schnittman, J. D. \& Krolik, J. H. 2009, ApJ, 701, 1175

Schnittman, J. D. \& Krolik, J. H. 2010, ApJ, 712, 908

Schödel, R., Krips, M., Markoff, S., Neri, R., \& Eckart, A. 2007, A\&A, 463, 551

Shahbaz, T., Hynes, R. I., Charles, P. A., Zurita, C., Casares, J., Haswell, C. A., Araujo-Betancor, S., \& Powell, C. 2004, MNRAS, 345, 31

Shafee, R., McClintock, J. E., Narayan, R., Davis, S. W., Li, L.-X., \& Remillard, R. A. 2006, ApJ, 636, L113

Shapiro, I. I., Davis, J. L., Lebach, D. E., \& Gregory, J. S. 2004, Phys. Rev. Lett., 92, 121101

Shcherbakov, R. V., \& Penna, R. F. 2011, ASP Conf. Ser., 439, 372

Shcherbakov, R. \& Penna, R. 2010 in The Galactic Center: a Window to the Nuclear Activity of Disk Galaxies, arXiv:0912.2106

Shibata, M., \& Sasaki, M. 1998, Phys. Rev. D, 58, 104011

Sibgatullin, N. R., Oscillations and Waves in Strong Gravitational and Electromagnetic Fields, Springer, 1991

Silbergleit, A. S., Wagoner, R. V., \& Ortega-Rodríguez, M. 2001, ApJ, 548, 335

Sopuerta, C. F. \& Yunes, N. 2009, Phys. Rev. D, 80, 064006

Speith, R., Riffert, H., \& Ruder, H. 1995, Comp. Phys. Comm. 88, 109

Srianand, R., Chand, H., Petitjean, P., \& Aracil, B. 2004, Phys. Rev. Lett., 92, 121302 
Stairs, I. H. 2003, Liv. Rev. Rel., 6, 5

Stella, L. 1990, Nature, 344, 747

Suen, W.-M., Price, R. H. \& Redmount, I. H. 1988, Phys. Rev. D, 37, 2761

Takahashi, R., \& Mineshige, S. 2011, ApJ, 729, 86

Takahashi, R. 2004, ApJ 611, 996

Tanaka, T. 2003, Prog. Theor. Phys. Suppl. 148, 307

Torres, M. A. P., Callanan, P. J., Garcia, M. R., Zhao, P., Laycock, S., \& Kong, A. K. H., 2004, ApJ, 612, 1026

Thorne, K. S. 1980, Rev. Mod. Phys., 52, 299

Tremaine, S., et al. 2002, ApJ, 574, 740

Valtonen, M. J., Mikkola, S., Lehto, H. J., Gopakumar, A., Hudec, R., \& Polednikova, J. 2011, ApJ, 742, 22

van der Klis, M. 2006 in Compact Stellar X-ray Sources, ed. W. Lewin \& M. van der Klis (Cambridge: Cambridge Univ. Press)

van Stockum, W. J. 1937, Proc. R. Soc. Edinburgh A, 57, 135

Vaughan, S., \& Uttley, P. 2005, MNRAS, 362, 235

Vaughan, S., \& Uttley, P. 2006, Adv. Space Res., 38, 1405

Verbunt, F. 1993, ARAA 31, 93

Vessot, R. F. C., et al. 1980, Phys. Rev. Lett., 45, 2081 
Viergutz, S. U. 1993, Astron. Astrophys., 272, 355

Vigeland, S. J. \& Hughes, S. A., 2010, Phys. Rev. D, 81, 024030

Vigeland, S. J. 2010, Phys. Rev. D, 82, 104041

Vigeland, S. J., Yunes, N., \& Stein, L. C. 2011, Phys. Rev. D, 83, 104027

Wagner, R. M., Foltz, C. B., Shahbaz, T., Casares, J., Charles, P. A., Starrfield, S. G., \& Hewett, P., 2001, ApJ, 556, 42

Wagoner, R. V. 1999, Phys. Rep., 311, 259

Wagoner, R. V., Silbergleit, A. S., \& Ortega-Rodríguez, M. 2001, ApJ, 559, L25

Wagoner, R. V. 2008, New Astron. Rev., 51, 828

Wald, R. M., General Relativity, University of Chicago Press, 1984

Webb, J. R., et al. 2000, AJ, 120, 41

Webbink, R. F., Rappaport, S., \& Savonije, G. J. 1983, ApJ, 270, 678

Weyl, H. 1918, Ann. Physik, 54, 117

Wex, N., \& Kopeikin, S. M. 1999, ApJ, 514, 388

Wild, W., et al. 2009, Ex. A., 23, 221

Will, C. M. 2009, Phys. Rev. Lett., 102, 061101

Will, C. M. 2008, ApJL, 674, L25

Will, C. M. 2006, Liv. Rev. Rel., 9, 3 
Will, C. M. 1993, Theory and Experiment in Gravitational Physics (Cambridge: Cambridge University Press)

Will, C. M., \& Zaglauer, H. W. 1989, ApJ, 346, 366

Williams, J. G., Turyshev, S. G., \& Boggs 2004a, D. H., Phys. Rev. Lett. 93, 261101

Williams, J. G., Turyshev, S. G., \& Murphy, T. W. 2004b, Int. J. Mod. Phys. D, 13, 567

Yuan, Y.-F., Cao, X., Huang, L., \& Shen, Z.-Q. 2009, ApJ, 699, 722

Yuan, F., Quataert, E., \& Narayan, R. 2003, ApJ, 598, 301

Yunes, N., \& Stein, L. C. 2011, Phys. Rev. D, 83, 4002

Yunes, N. \& Pretorius, F. 2009, Phys. Rev. D79, 084043

Yungelson, L, \& Lasota, J.-P. 2008, NewAR, 51, 860, arXiv:0801.3433v2

Yusef-Zadeh, F., Roberts, D., Wardle, M., Heinke, C. O., \& Bower, G. C. 2006, ApJ, 650,189

Zhang, S. N., Cui, W., \& Chen, W. 1997, ApJ, 482, L155

Zurita, C., Casares, J., Shahbaz, T., Wagner, R. M., Foltz, C. B., Rodríguez-Gil, P., Hynes, R. I., Charles, P. A., Ryan, E., Schwarz, G., \& Starrfield, S. G., 2002, MNRAS, 333, 791 